I N T ER N ATIONAL MONETARY FUND

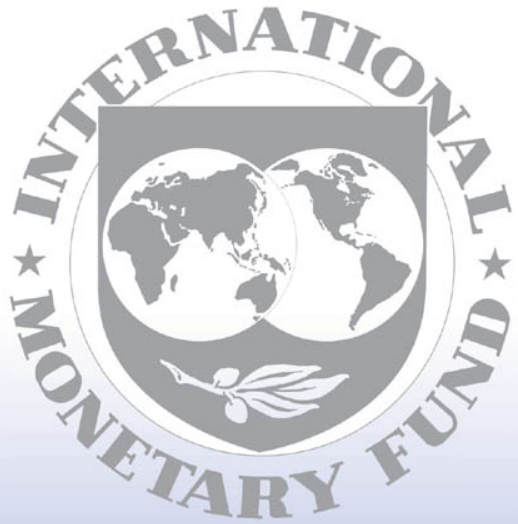

Staff

Country

Reports 


\section{Cameroon: Poverty Reduction Strategy Paper}

Poverty Reduction Strategy Papers (PRSPs) are prepared by member countries in broad consultation with stakeholders and development partners, including the staffs of the World Bank and the IMF. Updated every three years with annual progress reports, they describe the country's macroeconomic, structural, and social policies in support of growth and poverty reduction, as well as associated external financing needs and major sources of financing. This country document for Cameroon, dated April 2003, is being made available on the IMF website by agreement with the member country as a service to users of the IMF website.

To assist the IMF in evaluating the publication policy, reader comments are invited and may be sent by e-mail to publicationpolicy@imf.org.

Copies of this report are available to the public from

International Monetary Fund • Publication Services

$70019^{\text {th }}$ Street, N.W. • Washington, D.C. 20431

Telephone: (202) 623-7430 • Telefax: (202) 623-7201

E-mail: publications@imf.org • Internet: http://www.imf.org

Price: $\$ 15.00$ a copy

\section{International Monetary Fund \\ Washington, D.C.}


REPUBLIQUE DU CAMEROUN

Paix-Travail-Patrie
REPUBLIC OF CAMEROON

Peace-Work-Fatherland

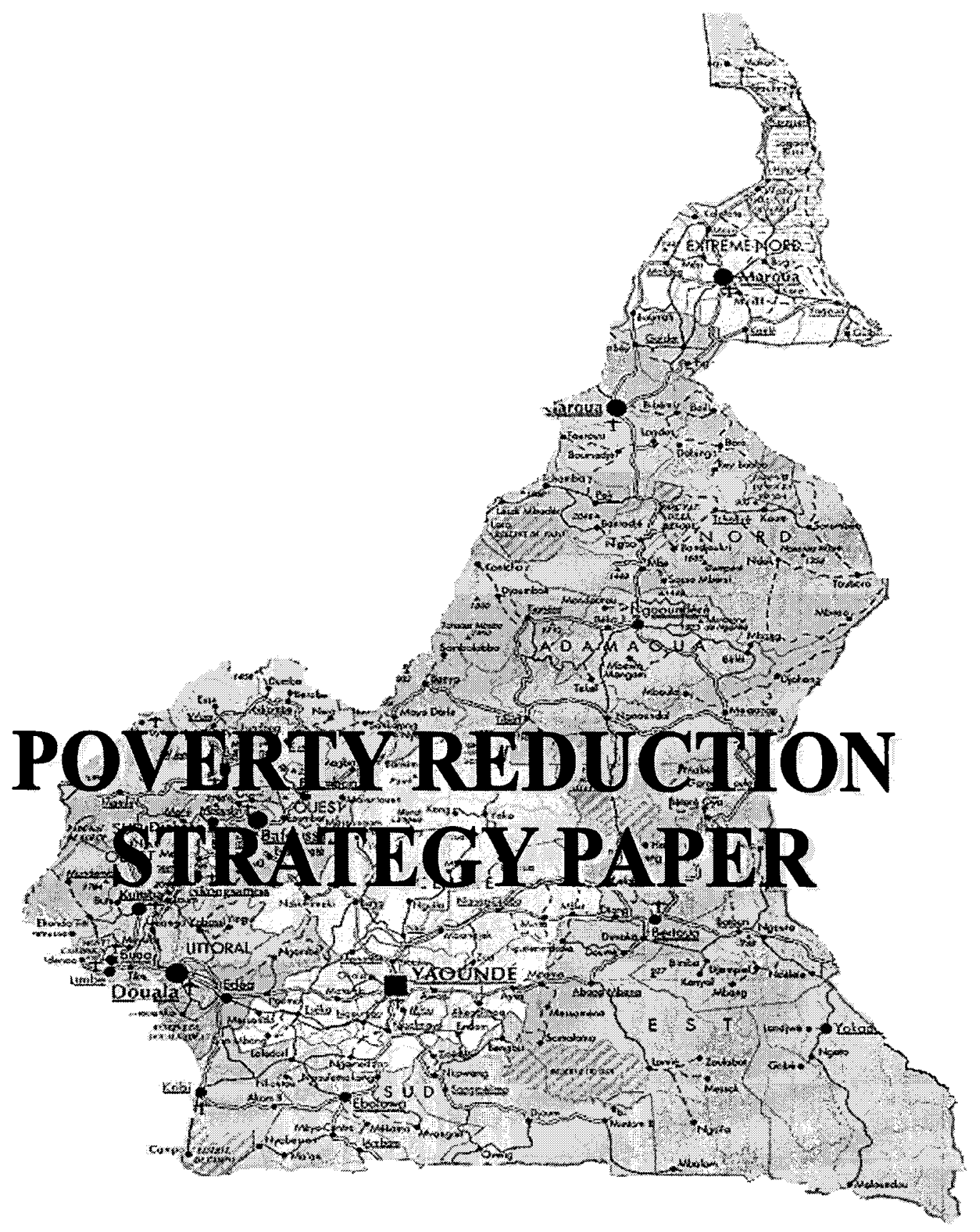

April 2003

CInternational Monetary Fund. Not for Redistribution 


\section{FOREWORD}

The adoption of the Poverty Reduction Strategy Document (PRSP) by the government of Cameroon is a milestone in the process of reforms carried out by the Government of Cameroon since October 2000, when the country reached the decision point of the Enhanced Initiative for Heavily Indebted Poor Countries (HIPC).

Indeed, thanks to the leadership of the Head of State, His Excellency Paul BIYA, and the adherence of the Cameroonians to his vision of a new society, Cameroon has successfully completed its first three-year economic and financial program (1997-2000), with the assistance of the international financial community. Today, there is a strong national commitment to continue and deepen the reforms needed to reach the HIPC completion point, which will provide the Government with the additional resources needed to sustain the fight against poverty.

The central objective of these reforms, which the Government is pursuing with determination, is to significantly reduce poverty, with a strong and sustainable economic growth, increased efficiency of public expenditure, more effective targeting of its poverty reduction policies and improvement in overall governance.

These policies are strategically framed in the PRSP and reflect the Government's ambition to attain the Millennium Development Goals. The Government has prepared the present document using an open and participatory process, which has involved the population at the grassroots level, the civil society, private sector, development partners and the public administration. On behalf of the government, I wish to take this opportunity to acknowledge their respective contributions and to express our gratitude for their willingness to participate and the quality of their work.

To finalize this document, the government has carried out several pieces of analytical work with the assistance of the World Bank and other development partners. These include a comprehensive study on the sources of growth, an analysis of poverty dynamics, and macromodeling work to align the priority sector medium term expenditure framework with the macroeconomic and budgetary framework. Both the PRSP and the IMF supported program under the Poverty reduction and growth facility (PRGF) share the same macroeconomic framework. In formulating this strategy, the Government has striven to respond to the needs expressed by the populations during the participatory consultations.

The PRSP has squarely placed poverty reduction at the center of Government's development policy. Henceforth, it will serve as the reference for all government actions and for coordinating donors' assistance. The PRSP will guide our efforts to achieve higher economic growth and a more equitable distribution of its fruits, including to the most vulnerable among our people.

Since August 2000, when the interim PRSP was transmitted to the IMF and the World Bank, the preparation of the full PRSP has been a learning process for all of its participants. The Government intends to continue using this participatory process to improve its poverty reduction strategy in future progress reports, revising as needed, policies and targets to reflect changes in the national and international environments. 
After having completed a quality PRSP, the Government now faces the challenge of ensuring its successful implementation. In this regard, the Government is resolute to rekindle efforts to accelerate the pace of reforms and ensure that improvements in economic performance translate into tangible social gains including job creation and improvement in the standard of living of the population.

As it addresses this challenge, the Government is counting on the dynamism of the private sector, the involvement of civil society, the mobilization of the population and the support of the donor community.

THE PRIME MINISTER,
HEAD OF GOVERNMENT,

Peter MAFANY MUSONGE 
AER

AFD

AfDB

AGOA

ANTIC

APECCAM

ARMP

ARSEL

ARV

BEAC

BICEC

BOO

BOT

BTP

C2D

CAEMC

CAMCCUL

CAMTEL

CAR

CAS III

CDC

CDT

CFAA

CNPS

COBAC

CODE CIMA

COOPEC

CSI

CTS

CV

DME

DP

DSCN
Agence d'Electrification Rurale (Rural electrification)

Agence Française de Développement (French Development Assistance)

African Development Bank

American Growth Opportunity Act

Agence Nationale des Technologies de l'Information et de la Communication

(Information and communication technologies agency)

Association Professionnelle des Etablissements de Crédits du Cameroun

(Credit Unions Association)

Agence de Régulation des Marchés Publics (Procurement Regulatory Agency)

Agence de Régulation du Secteur de l'Electricité (Electricity Regulatory

Agency)

Anti-retrovirals

Bank of Central African States

Banque Internationale du Cameroun pour l'Epargne et le Crédit

Build Own and Operate

Build Operate and Tranfer

Bâtiments et Travaux Publics (Construction and Public Works)

Contrat de Désendettement et Développement (French Debt cancellation

Initiative)

Central African Economic and Monetary Community

Cameroon Cooperative Credit Union League

Cameroon Telecommunications (Société Camerounaise des

Télécommunications)

Central African Republic

Troisième Crédit d'Ajustement Structurel de l'Association Internationale pour le Développement (Third Structural Adjustment Credit, under IDA financing)

Cameroon Development Corporation

Centre de Diagnotic-Traitement (Screening and Treatment Center)

Country Financial Accountability Assessment

Caisse Nationale de Prévoyance Sociale

Commission Bancaire de l'Afrique Centrale (Central Africa Banking

Commission)

Code de la Conférence Interafricaine du Marché des Assurances (Inter-African

Insurance Association)

Coopératives d'Epargne et de Crédit (Saving and Loans Cooperatives)

Centre de Santé Intégré (Integrated health centers)

Comité Technique de Suivi des Programmes Economiques (Economic

program monitoring committee)

Caisse Villageoise (Village-level fund)

Minimum diameter (of a tree)

Direction de la Prévision (Economic Planning Directorate)

Direction de la Statistique et de la Comptabilité Nationale (National Accounts 


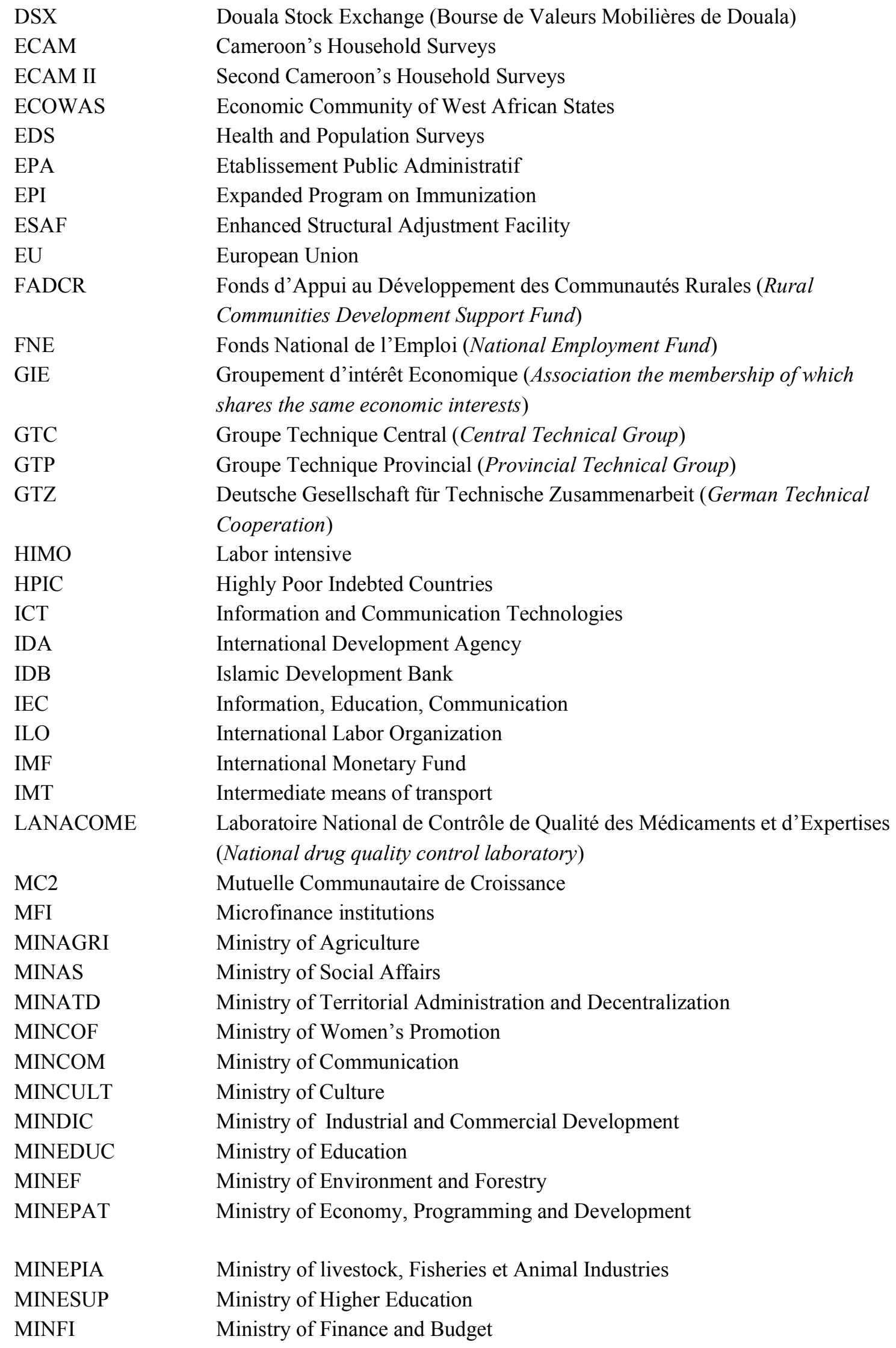




\begin{tabular}{|c|c|}
\hline MINMEE & Ministry of Mines, Water and Power \\
\hline MINPOSTEL & Ministry of Postal Services and Telecommunications \\
\hline MINREST & Ministry of Scientific and Technical Research \\
\hline MINSANTE & Ministry of Public Health \\
\hline MINTOUR & Ministry of Tourism \\
\hline MINTP & Ministry of Public Works \\
\hline MTEF & Medium Term Expenditure Framework \\
\hline NGO & Non-Governmental Organization \\
\hline NTFP & Non Timber Forest Products \\
\hline ODA & Official Development Assistance \\
\hline OPCVM & Stock exchange related institution \\
\hline \multirow[t]{2}{*}{ PADC } & Programme d'Appui au Développement Communautaire (Community \\
\hline & Development Support Program) \\
\hline PCIME & Prise en Charge Intégrée des Maladies de l'Enfant \\
\hline \multirow[t]{2}{*}{ PNDP } & Programme National de Développement Participatif (National Community \\
\hline & Driven Development Program) \\
\hline PNE & Politique Nationale de l'Emploi (National Employment Policy) \\
\hline PNG & Programme National de Gouvernance (National Governance Program) \\
\hline PPMF & $\begin{array}{l}\text { Programme d'Appui au Programme National de la Microfinance (National } \\
\text { Microfinance Support Program) }\end{array}$ \\
\hline PRGF & Poverty Reduction and Growth Facility \\
\hline PSFE & Forestry \& Environment Sector Program \\
\hline PSM & Core Statistics Program \\
\hline PVVS & Personne Vivant avec le virus du SIDA \\
\hline RGPH & General Population and Housing Census \\
\hline RNRP & $\begin{array}{l}\text { Réseau National de Réduction de la Pauvreté (National Poverty reduction } \\
\text { Network) }\end{array}$ \\
\hline SGDD & $\begin{array}{l}\text { Système Généralisé de Diffusion des Données (Generalized Data } \\
\text { Dissemination System) }\end{array}$ \\
\hline SIC & Société Immobilière du Cameroun (Real Estate Company) \\
\hline SICAV & Société Immobilière à Capital Variable (Mutual Funds) \\
\hline SIGEFI & $\begin{array}{l}\text { Système d'Information Intégré pour la Gestion des Finances Publiques } \\
\text { (Integrated Public Finance Management System) }\end{array}$ \\
\hline SIGIPES & Système Intégré de Gestion du Personnel de l'Etat et de la Solde (Integrated \\
\hline & Civil Service Management and Payroll System) \\
\hline SME & Small and Medium Scale Enterprises \\
\hline SMI & Small and Medium Scale Industries \\
\hline SNEC & Société Nationale des Eaux du Cameroun (National Water Utility) \\
\hline SNH & Société Nationale des Hydrocarbures (National Oil Company) \\
\hline SONEL & Société Nationale d'Electricité (National Power Utility) \\
\hline SYNAME & $\begin{array}{l}\text { Système National d'Approvisionnement en Médicaments, réactifs et } \\
\text { consommables médicaux (National drug, reagents and medical supplies } \\
\text { procurement system) }\end{array}$ \\
\hline TBS & Social indicators management reporting system \\
\hline UNDP & United Nations Development Program \\
\hline UNESCO & United Nations Educational, Scientific and Cultural organization \\
\hline
\end{tabular}


UNFPA United Nations Population Fund

UNICEF United Nations Children's Funds

UNIDO

United Nations Industrial Development Organization

WB

World Bank

WTO

World Trade Organization 
TABLE OF CONTENTS

EXECUTIVE SUMMARY

CHAPTER 1 : RECENT DEVELOPMENTS AND BACKGROUND TO THE PRSP ...................................... 1

1.1 INTRODUCTION ..................................................................................................................................................

1.2 THE MACROECONOMIC SITUATION................................................................................................................................

1.3 THE SOCIOECONOMIC SITUATION.................................................................................................................................

1.4 THE CONTEXT AND FEATURES OF CAMEROON'S PRSP ........................................................................................ $\underline{5}$

CHAPTER 2 : THE CHARACTERISTICS OF POVERTY IN CAMEROON................................................... $\underline{8}$

2.1 DEFINITIONS AND PERCEPTIONS OF POVERTY .................................................................................................................. 9

2.2 THE QUANTITATIVE OR MONETARY APPROACH TO POVERTY ..................................................................................... 9

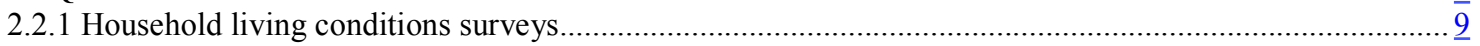

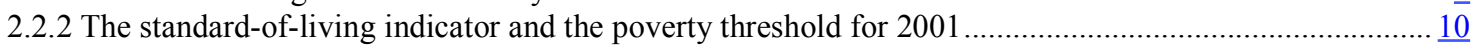

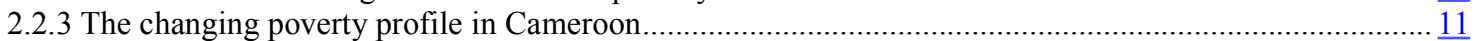

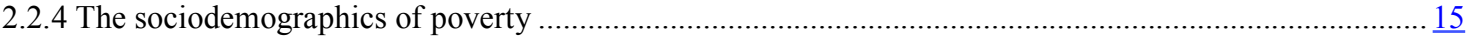

2.2.5 The determinants of poverty ........................................................................................................ $\frac{17}{17}$

2.3 PARTICIPATORY ASSESSMENT OF POVERTY IN CAMEROON ............................................................... 19

2.3.1 The main stages of the participatory process for preparing the PRSP ....................................................

2.3.2 How people perceive poverty......................................................................................................... 20

2.3.3 How people see the determinants of poverty...............................................................................

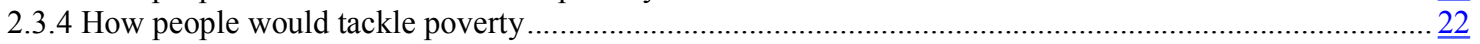

2.3.5 Monitoring and assessment mechanisms favored during the consultations ……………………………... $\underline{23}$

2.4 THE OTHER DIMENSIONS OF POVERTY AND HUMAN DEVELOPMENT IN CAMEROON ……………………………... $\underline{23}$

2.4.1 Education, training and poverty reduction …………...............................................................

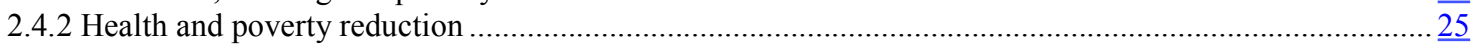

2.4.3 Basic infrastructure services...................................................................................................... $\underline{28}$

CHAPTER 3 : GROWTH AND POVERTY REDUCTION STRATEGY ......................................................

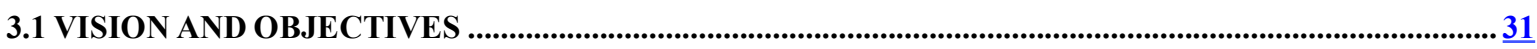

3.2 A MACROECONOMIC FRAMEWORK THAT CREATES THE CONDITIONS FOR GROWTH.......... $\underline{\underline{33}}$

3.3 CONSOLIDATING GROWTH BY DIVERSIFYING THE ECONOMY ……………………….................... 34

3.3.1 Rural sector: a key sector for economic growth ........................................................................ $\frac{35}{\underline{35}}$

3.3.2 Industrialization to enhance and stabilize growth ............................................................................... $\frac{46}{46}$

3.3.3 Developing production support services with high value-added........................................................... $\frac{50}{55}$

3.4 REVITALIZING THE PRIVATE SECTOR ……….......................................................................................... $\frac{55}{5}$

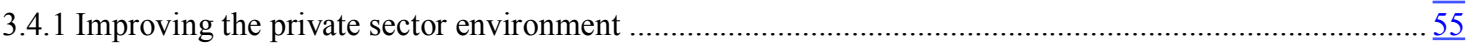

3.4.2 Financial intermediation in support of the private sector .................................................................. $\frac{57}{59}$

3.5 INFRASTRUCTURE, NATURAL RESOURCES, AND ENVIRONMENT …………………....................... 59

3.5.1 Transportation infrastructure ................................................................................................. $\frac{59}{63}$

3.5.2 Construction and public works...................................................................................................... $\frac{63}{63}$

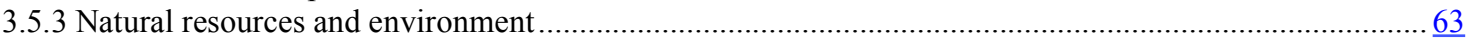

3.6 ACCELERATING REGIONAL INTEGRATION WITHIN CAEMC ……………….................................... $\underline{66}$

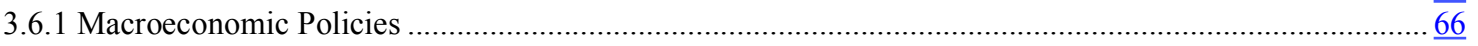

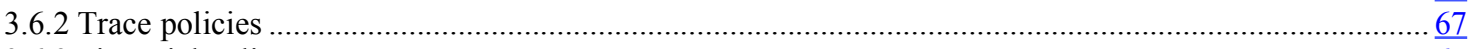

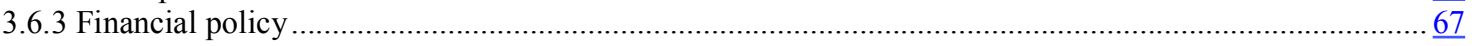

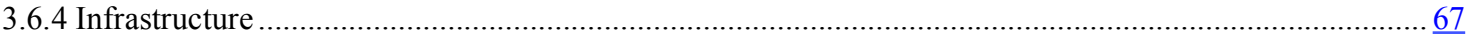

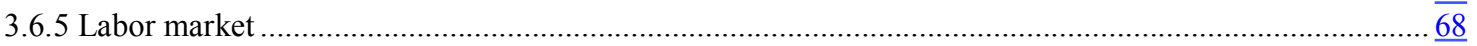

3.6.6 Areas for sector cooperation.........................................................................................................

3.7 STRENGTHENING HUMAN RESOURCES AND THE SOCIAL SECTOR................................................ $\frac{69}{69}$

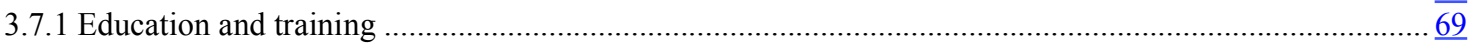

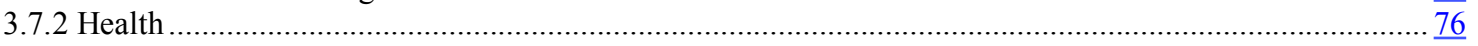

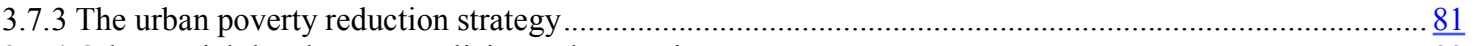

3.7.4 Other social development policies and strategies................................................................................ 83

3.7.5 Job creation and the integration of disadvantaged groups into the economy …………........................... $\underline{85}$

3.8 IMPROVING GOVERNANCE ……………………............................................................................................. $\frac{87}{87}$

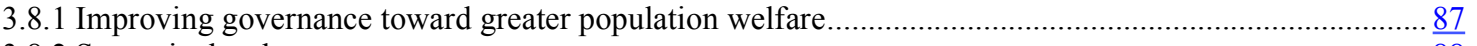

3.8.2 Strategic development management....................................................................................................... $\frac{88}{89}$

3.8.3 Motivating and mobilizing Cameroon's active forces around a common vision ....................................... $\underline{89}$ 
3.8.4 Enforcing the rule of law and ensuring the security of goods and persons ....................................... $\underline{89}$

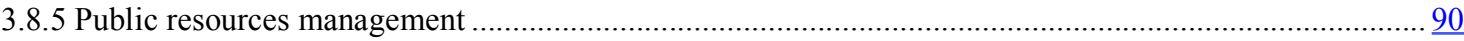

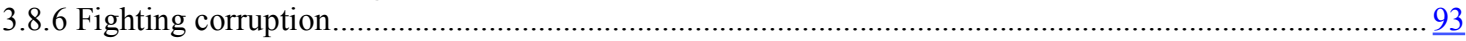

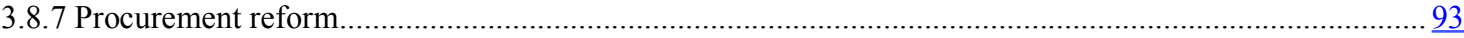

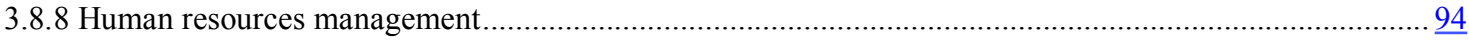

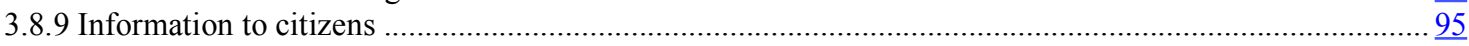

CHAPTER 4 : MACROECONOMIC AND SECTOR FRAMEWORK .......................................................... 96

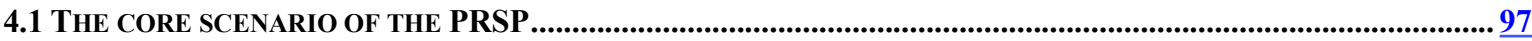

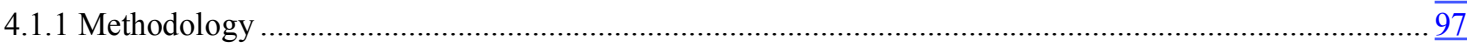

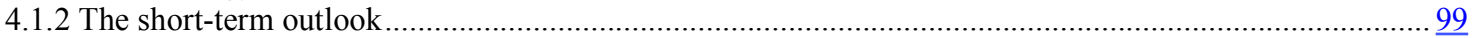

4.1.3 The sectoral strategies and medium-term growth potential ......................................................... 99

4.1.4 The medium-term growth outlook by branch of activity........................................................ 101

4.1.5 The medium-term macroeconomic and financial framework ....................................................... 103

4.1.6 The medium-term budget framework (MTBF) .................................................................... 106

4.1.7 The impact of growth and the distribution of growth on the poverty rate .....................................

4.2 SECTORAL STRATEGY FRAMEWORK PROJECTIONS (CADRAGES) AND CONSISTENCY WITH THE PRSP ...........111

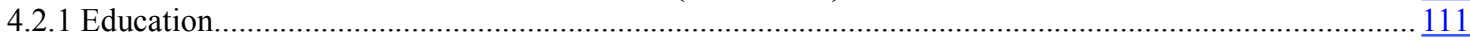

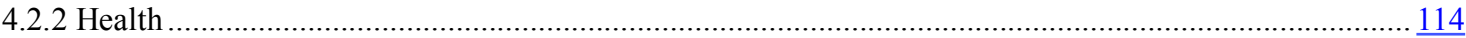

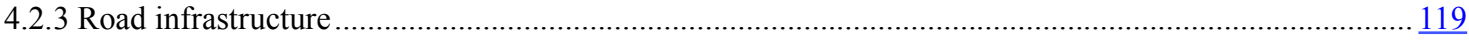

4.3 FINANCING THE POVERTY REDUCTION STRATEGY

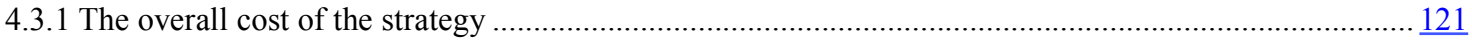

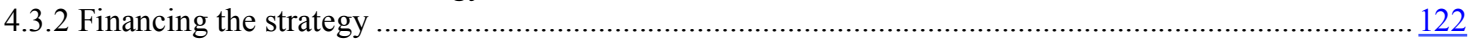

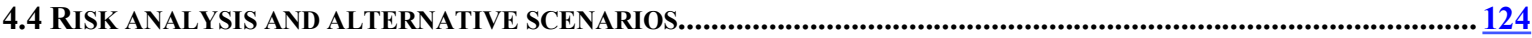

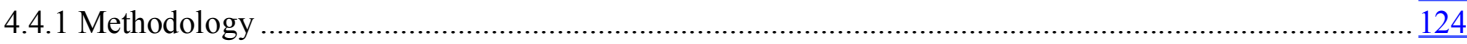

4.4.2 Impact of adverse shocks on the terms of trade........................................................................ 124

4.4.3 Effects of a contraction in the expected flow of funds from abroad or of persisting fund absorption

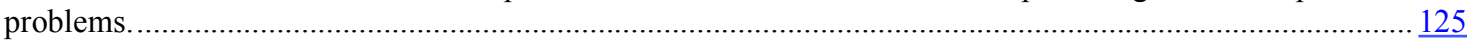

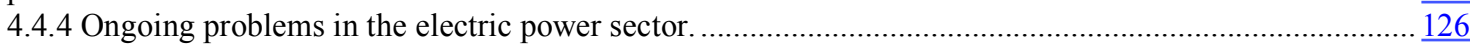

CHAPTER 5 : THE INSTITUTIONAL FRAMEWORK AND MECHANISMS FOR IMPLEMENTING

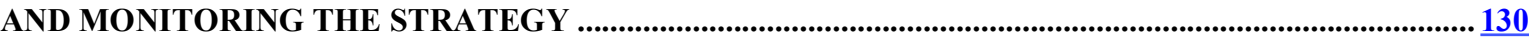

5.1 STEERING AND OVERSEEING IMPLEMENTATION OF THE STRATEGY .............................................................131

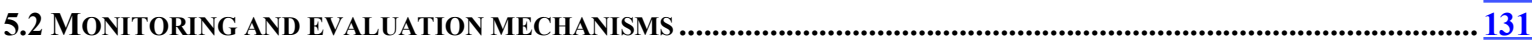

5.3 IMPROVEMENTS IN THE PRODUCTION AND DISSEMINATION OF STATISTICAL INFORMATION ..........................132

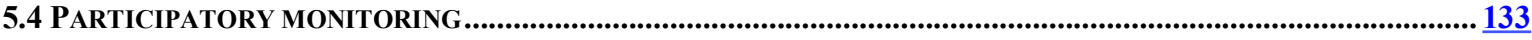

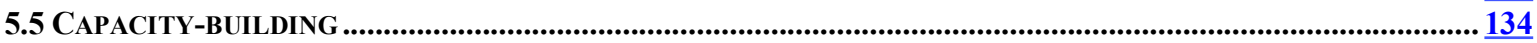

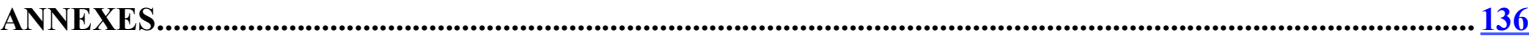

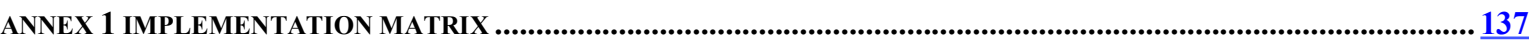

ANNEX 2 CORE POVERTY MONITORING INDICATORS AND STATISTICAL PROGRAM ............................................... 158

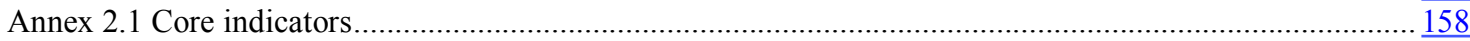

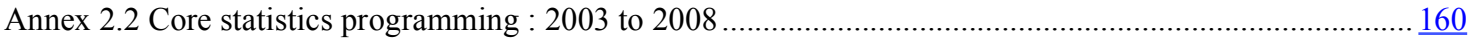

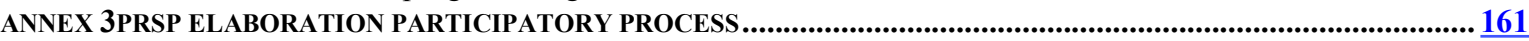

Annex 3.1 Major stages of PRSP elaboration and participatory process ............................................. 161

Annex 3.2 Definition of poverty by different population groups during the participatory consultations.......... $\underline{163}$

Annex 3.3 Poverty reduction actions and strategies derived from the participatory consultations .................. 167

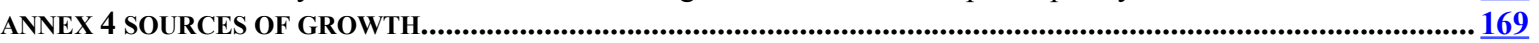

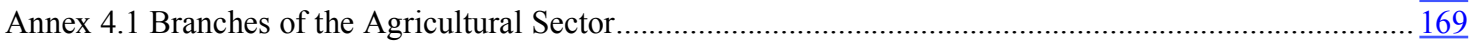

Annex 4.2 Key branches of the industrial sector.......................................................................... 172

ANNEX 5 MEDIUM-TERM MACROECONOMIC FRAMEWORK................................................................................179

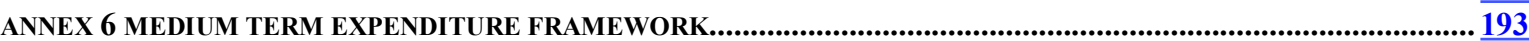

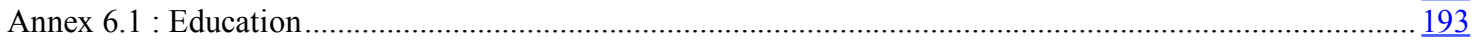

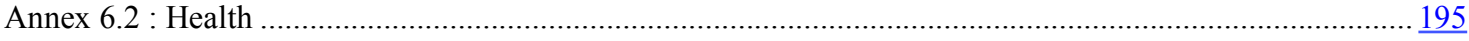

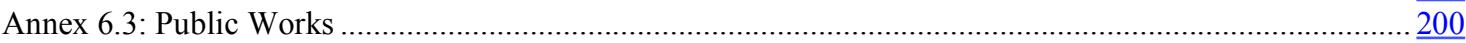

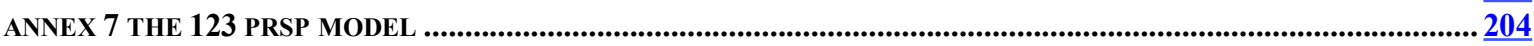

ANNEX 8 RESULTS OF POVERTY DETERMINANTS ECONOMIC MODEL .................................................................206 


\section{GRAPHS AND FIGURES}

Graph 1. The prevalence of hiv/aids in cameroon (population ages 15 to 49 ) ……….........................................

Figure 1. Objectives, priorities, and programs for the education strategy, by sector …………………………....

Graph 3. Percentage completing primary school, depending on strategy options ................................................113

Graph 4. Expenditure on education in the mdgs and prsp scenarios: a comparison............................................113

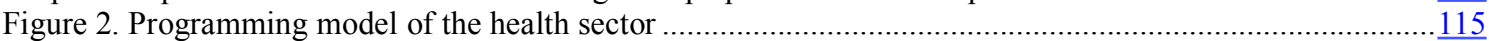

Figure.3. Methodological framework for planning ..................................................................................

\section{TABLES}

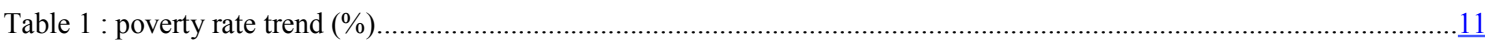

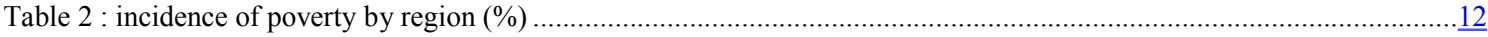

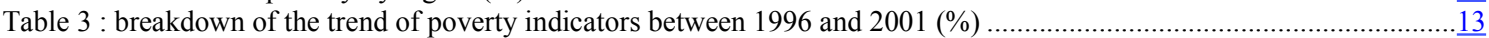

Table 4 : income inequalities : gini index and average expenditure by quintile .........................................................14

Table 5 : poverty incidence by sex and age of household head $(\%)$

Table 6 : literacy of household head and children's school enrollment $(\%)$

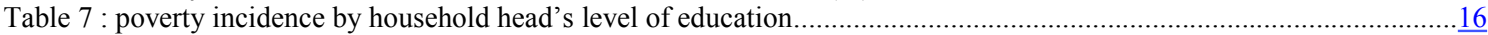

Table 8 : poverty incidence by household head's occupational category ……..........................................................16

Table 9 : variation in poverty incidence following a 5\% change in the poverty threshold ..............................................17

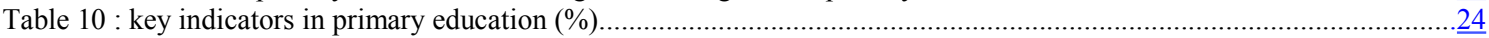

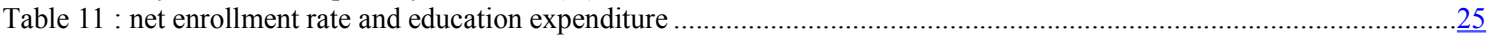

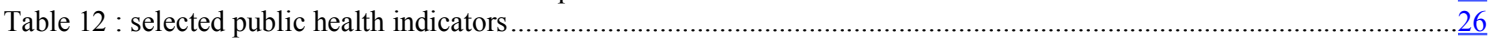

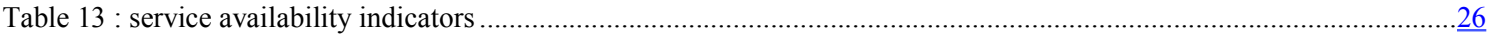

Table 14 : consultation and immunization rates and health expenditures .....................................................................27

Table 15 : access to drinking water, electricity and other basic infrastructure ….........................................................28

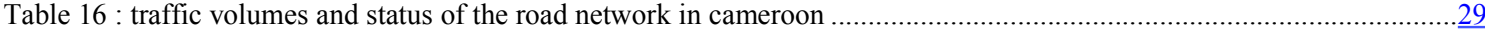

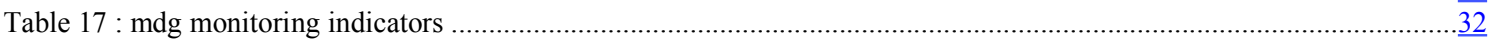

Table 18 : millennium development goals in education $(\%)$....................................................................................

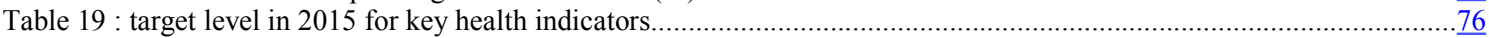

Table 20 : impact of hiv/aids on gdp growth in some african countries ............................................................... 100

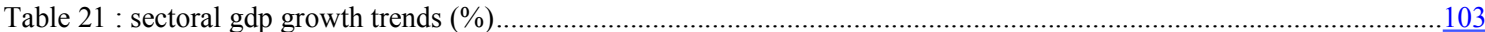

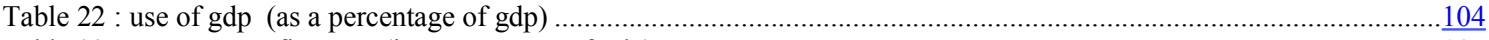

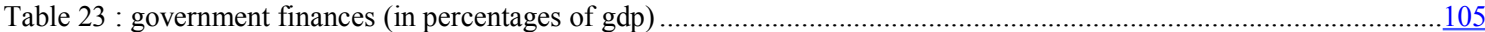

Table 24 : financing and debt (in percentages of gdp) ......................................................................................

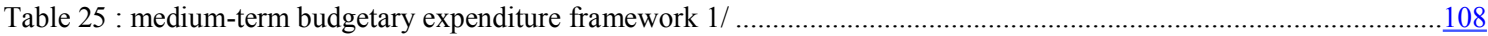

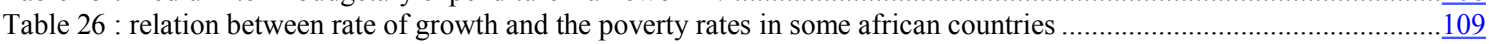

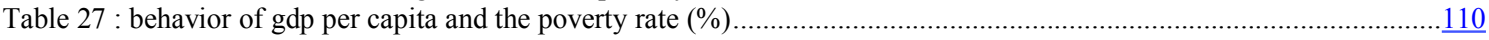

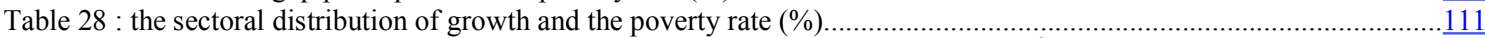

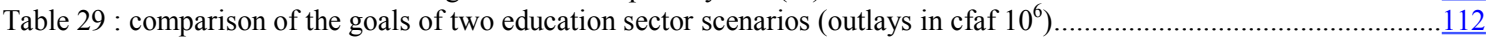

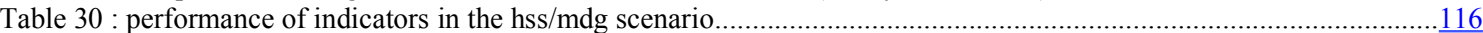

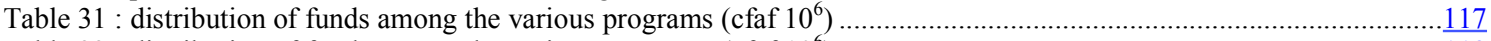

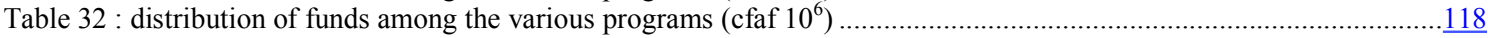

Table 33 : summary of how far each scenario falls short of the millennium development goals ..................................... 119

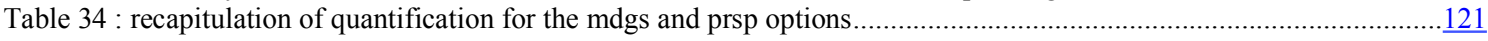

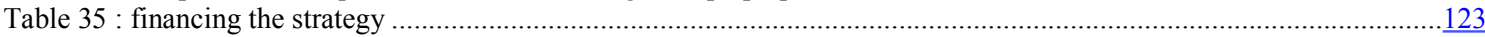

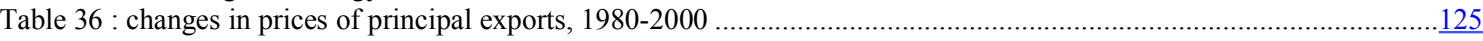

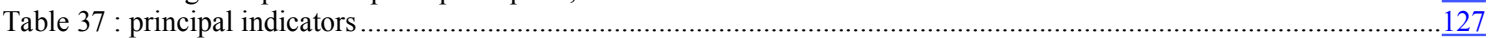

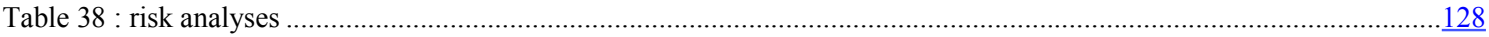

\section{BOXES}

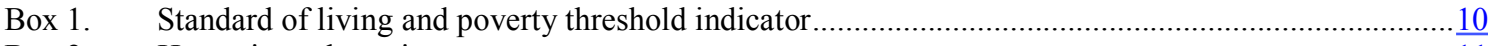

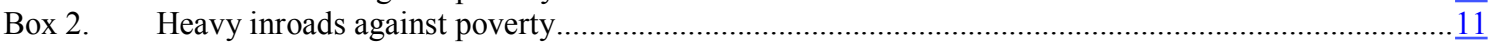

Box 3. The decline of poverty : the growth effect and the redistribution effect........................................13

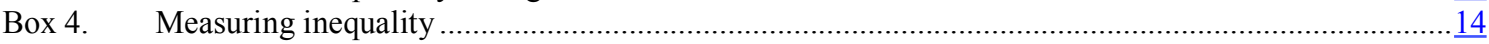

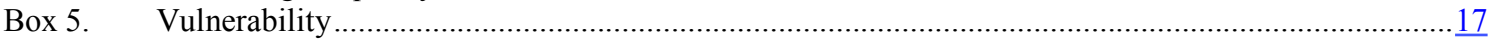

Box 6. Identifying the principal determinants of poverty in cameroon..................................................

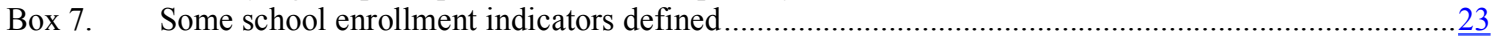

Box 8. Studies of competitiveness and sources of growth in cameroon's economy ……..........................

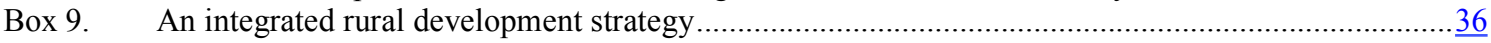

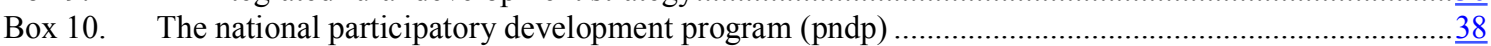

Box 11. The marketing cost of agricultural products: food crops ...................................................... 
Box 12. Comparative advantages of agricultural subsectors.......................................................... 40

Box 13. The forestry environment sector program (psfe) ….......................................................... 44

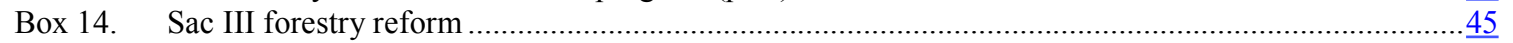

Box 15. The comparative advantage of industrial subsectors .............................................................

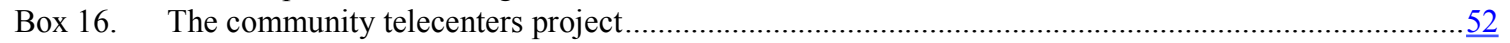

Box 17. A single processing window for external trade..................................................................

Box 18. The national microfinance program support project.......................................................

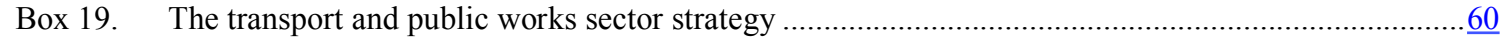

Box 20. Rural road maintenance and rehabilitation strategy................................................................

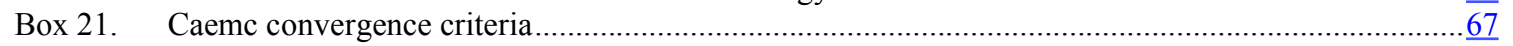

Box 22. Financial cost of the status quo policy in the education sector ...............................................

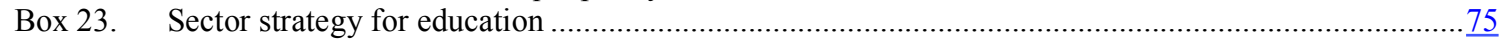

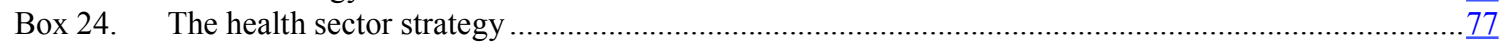

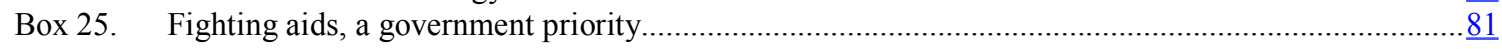

Box 26. Status of the rural employment development support program ................................................ 8

Box 27. National governance program: objectives and main priorities of the priority action plan ................. $\underline{88}$

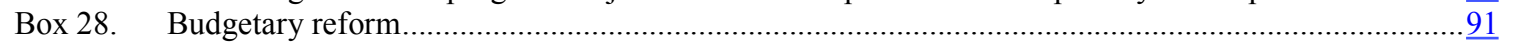

Box 29. Brief description of the macro-econometric model ….......................................................... 98

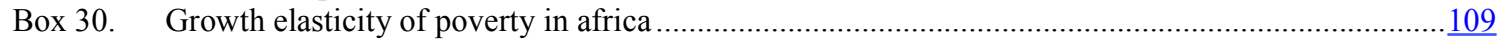

Box 31. The financial simulation model for the education sector ..................................................... 114

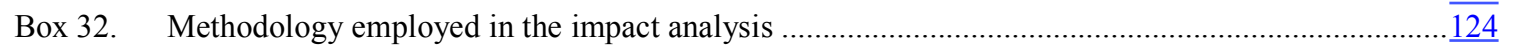




\section{EXECUTIVE SUMMARY}

1. This poverty reduction strategy document has been designed and prepared using a participatory approach that closely involved participants from public administration, private operators, civil society as well as development partners at all stages. The document comprises five chapters. Chapter one provides an overview of recent economic and social developments in Cameroon, which serves as a background for the PRSP. Chapter two analyzes the state and the dynamics of poverty in Cameroon, which serves as a guide to government poverty reduction policy choices and strategy formulation. Chapter three describes the pillars of the short and medium-term growth and poverty reduction strategy. Chapter four provides a quantitative assessment and costing of Cameroon's PRS, including simulation of the short and medium-term GDP growth profile and the related medium-term expenditure framework, a consistency check between the macroeconomic framework and sector strategies, and estimates of the total cost of the strategy. Chapter five explains how the PRS will be monitored and evaluated in a participatory manner. The PRSP also provides various annexes, including a matrix of planned priority actions as well as tables of indicators for monitoring.

\section{Introduction: Recent Developments and Background of the PRSP}

2. Background. At the dawn of the third millennium, Cameroon is at crossroads: it is endowed with significant assets, but it also faces major challenges: to diversify its economy, consolidate growth, and improve the standard of living of its population. Cameroon's many assets include a stable macroeconomic framework - the result of sustained adjustment efforts, an improved business environment that is conducive to private sector development, an enviable position as a development pole within an increasingly open and integrated subregion, a relatively young and well-educated population that is capable of absorbing new technologies and raising productivity, and a stable political and institutional environment. These assets constitute an important stock of "social capital" that can help Cameroon attract foreign investment, diversify the economy, and intensify growth in line with the needs and expectations of its population.

3. Cameroon needs this "social capital" in order to overcome an important "social deficit" which, if left unattended, will weaken the foundations for sustainable growth and undermine the country's social fabric. Despite important gains during the second half of the 1990s, nearly four out of every ten Cameroonians in 2001 were living with an annual income below the poverty line of CFAF 232,547 (roughly equivalent to US\$1 per person, per day, or FCFA 19,000 per month), which represents the estimated annual income necessary for an individual in Yaoundé to buy a "minimal basket" of essential food and non-food items, including health, education, and housing expenditures. Overall, indicators of human development have considerably deteriorated during the crisis years, particularly in education and health, and recent economic improvements have not yet been sufficient and sustained enough to fully remedy the situation, even if the incidence of poverty has begun to regress.

4. In education, access to primary school rose to 95 percent in 2001, partly due to a government decision to eliminate public school fees. However, the primary school completion rate has remained low (56 percent), indicating that only one out of every two children entering primary school manages to successfully complete sixth grade because of high rates of repetition ( 25 percent) and drop-out. Also, only 60 percent of students who complete primary school make a successful transition to secondary education. These weak completion and retention rates are indicative of underlying structural problems, and they have considerable social and economic costs that are rising with rapid population growth. Furthermore, the general health of the Cameroonian population has deteriorated considerably since the early 1990s. The child mortality rate, especially, increased by 12 percentage points between 1991 and 1998, chronic malnutrition rate for children 12 to 23 months old also rose from 23 percent to 29 percent, and the rate of delivery attended by qualified practitioners (doctors or nurses) declined by 5 percent during the same period. Moreover, between 1991 and 2002, the rate of HIV/AIDS infection 
within the sexually active population (people between the ages of 15 and 49) climbed alarmingly from 2 percent to 11.8 percent. At the same time, essential infrastructures have deteriorated considerably with the crisis, implying that the supply of road services, electricity, and drinking water is inadequate to meet the needs of the population and the exigencies of sustained economic growth.

5. Those problems have become more acute with the rapid growth (5 percent) of Cameroon's urban population. Furthermore, its population is relatively young (42 percent are less than 14 years and 72 percent less than 30 years old), and the young population is heavily concentrated in urban areas. While this may constitute an asset for the economy, it also places considerable pressure on social services, infrastructure, and the labor market. Without adequate and sustained attention by the government, these developments may transform major urban centers into breeding grounds for poverty and insecurity, eroding Cameroon's social capital and undermining the very competitive assets it needs to integrate fully into the global economy.

6. The Government of Cameroon (GOC) is well aware of this situation and determined to mobilize the living strength of the country in support of an ambitious but realistic strategy for broad-based human development in Cameroon. To this end, the GOC has prepared a new generation of economic and social reforms that aim to consolidate the achievements of previous programs and stimulate economic growth while also shoring up the social sector. Such a strategy is intended to ensure that enhanced economic growth also translates into real improvements in the living conditions of the population, especially the poor. In August 2000, the GOC took an important step in this direction by preparing an Interim Poverty Reduction Strategy Paper (I-PRSP). Implementation of the I-PRSP was accompanied by a series of specific strategy documents focusing on priority sectors such as education, health, and rural areas as well as essential infrastructures (roads, water); preparation of a social development strategy document is also underway. The now-completed PRSP represents a culminating point in this process.

7. The Main Characteristics and Functionalities of the PRSP. The GOC intends to use the Poverty Reduction Strategy Paper (PRSP) as a framework for blending a new generation of economic and social policies into a coherent set for accelerating growth and fighting poverty in a sustainable fashion. The PRSP is an evolving document that will be fine-tuned as new sector strategies are being prepared and implemented. The government intends to strengthen the PRSP's characteristics and functionality in future versions. Cameroon authorities consider the PRSP to serve as:

- An integrated development framework for Cameroon, which is articulated around macroeconomic and sector strategies for accelerating growth, reducing poverty, and helping Cameroon achieve the Millennium Development Goals (MDG)

- A consultation mechanism bringing together civil society and development partners to discuss major directions in economic and social policies as well as in development strategy management

- A framework for coordinating government programs and donors assistance strategies, including setting priorities, efficiently coordinating the actions of various branches of the administration and the Government, and mobilizing internal and external resources towards achieving Cameroon's sustainable human development objectives

- A medium-term consistency framework for setting budgetary priorities and allocating resources accordingly, while ensuring consistency between resources derived from economic growth or external assistance and the short and medium-term financing requirements of priority sector programs

- A framework for defining and organizing important analytical work programs and strengthening capacities for guiding development management, including statistical work for monitoring the PRS and economic modeling work for preparing medium-term macroeconomic and sector frameworks and performing poverty and social impact analyses. 


\section{Poverty in Cameroon}

8. In the preparation of the PRSP, Cameroon's government commissioned analytical work to assess poverty and identify its characteristics and determinants. The first in-depth quantitative analysis used the results of two major household surveys (ECAM I, 1996, and ECAM II, 2001). The analysis was complemented by a qualitative evaluation of poverty and its determinants, based on participatory consultations with the population across the entire country.

9. The quantitative analysis shows that: (i) the incidence of income poverty in Cameroon remains high, affecting 40.2 percent of the population in 2001, which does, however, constitute a marked 13.1 percent improvement over the 1996 level of 53 percent; (ii) poverty in Cameroon varies considerably according to regions, roughly increasing twofold between urban (22 percent) and rural areas (50 percent); and (iii) the poverty rate is higher for farmers (57 percent) and informal rural sector operators (54 percent), as well as for informal sector workers or the unemployed in urban areas (40 percent). The analysis also highlights the importance of education and infrastructure services in explaining poverty. It shows that one out of two poor people live in a household where the main income provider does not have a primary education and access to basic social services (education, health, water, roads) is more difficult for the poor than for the non-poor.

10. An analysis of poverty dynamics indicates that growth has contributed 11.8 percent of the 13percent decline in poverty incidence between 1996 and 2001, whereas redistribution has accounted for less than 2 percent. Finally, a quantitative analysis of the determinants of poverty confirms the importance of such factors as the agro-economic region, the sector of economic activity, education level, and the availability of and access to infrastructure services in Cameroon.

11. The main conclusions of the quantitative analysis are supported by findings from the participatory consultation with the population, which was organized by the authorities. In particular, this qualitative approach reveals that Cameroon's population perceives poverty as primarily a condition of material deprivation that is characterized by: (i) insufficient resources for meeting essential needs and (ii) poor access to basic infrastructure services such as water, roads, and electricity and to social services such as health and education. The population also perceives poverty as the result of a weakening social value system that translates in moral deprivations, loss of self-esteem, loosening family ties and a weakening sense of family solidarity, as well as widespread ethnic biases and social discrimination. Finally, the population also associates poverty with insecurity, a lack of protection against abuses, and a lack of basic rights and access to essential legal services.

12. The participatory consultations have also helped the authorities to collect valuable recommendations from the population regarding key strategic areas and priority actions for the poverty reduction strategy. In particular, Cameroonian populations have stressed the overriding need for public actions designed to improve their capacity to generate economic activities and "to take their development in their own hands," including government support to the rural sector and to facilitate the insertion both of the poor and young into the economy through well-targeted actions. They also emphasized the need to improve access to water, particularly in the country's northern region, and to develop roads in order to integrate all regions and the poor into mainstream economic activities, including production and trade. They have recommended that the government pay more attention and devote adequate resources to improving education and combating contagious diseases, particularly the HIV/AIDS pandemic and malaria, which together account for most of Cameroon's high morbidity and mortality rates.

\section{The Key Strategic Areas of the PRS}

13. The main conclusions of the previous analyses have helped the GOC to identify seven mediumterm strategic focus areas for attacking poverty, in line with Cameroon's key development objectives: 
- Priority 1: promoting a stable macroeconomic framework;

- Priority 2: strengthening growth by diversifying the economy;

- Priority 3: revitalizing the private sector as the main engine of growth and a partner in delivering social services;

- Priority 4: developing basic infrastructures and natural resources while protecting the environment;

- Priority 5: accelerating regional integration in the framework of CEMAC;

- Priority 6: strengthening human resources and the social sector and facilitating the integration of vulnerable groups into the economy;

- Priority 7: improving the institutional framework, administrative management, and governance.

\section{Strategic Area 1: Promoting a stable macroeconomic environment}

14. After several inconclusive attempts, Cameroon satisfactorily implemented an economic and financial program supported by the International Monetary Fund (IMF) under the Enhanced Structural Adjustment Facility (ESAF) from July 1, 1997, to June 30, 2000. The program aimed to consolidate the governments fiscal position and create conditions for sustained economic growth that translates into significant improvements in the livelihood of the population. The satisfactory execution of this program has contributed the progressive restoration of Cameroon's credibility within the international financial community. As a result, on October 1, 2000, the government committed to a second 3-year economic and financial program supported by the IMF under the Poverty Reduction and Growth Facility (PRGF). More than its predecessor, this second program focuses on poverty reduction and is expected to be concluded by the end of 2003.

15. Thanks to these programs and especially to the concomitant structural reforms undertaken by the GOC, Cameroon's economy has returned to macroeconomic stability, with GDP growth around 4.7 percent per year between 1997 and 2001 and an inflation rate averaging 3 percent during the same period. A key objective of the PRS is to consolidate these important gains and maintain a stable and growth-enhancing macroeconomic framework. In line with the PRGF, the government's short and medium-term objectives are to prudently raise GDP growth from 4.5 percent in 2002 to around 5 percent between 2003 and 2004 while reducing the rate of inflation from 3 percent to 2 percent. In public finance, the main objective is to increase non-oil revenues from 15.6 percent of the non-oil GDP in 2002 to 16.4 percent in 2003 and to limit the overall budget deficit on commitment base to less than 3 percent of GDP. The planned reimbursement of internal debt should increase household savings and shore up private investments. This, combined with increasing public expenditures during the first years of the PRS, would help raise the overall investment rate from 18 percent to around 20 percent of GDP by 2004 in support of growth.

\section{Strategic Area 2: Diversifying the economy for accelerated growth}

16. The government is well aware that the pace of growth during the past few years will not be sufficient to provide Cameroon with adequate resources to bridge the "social deficit," as envisioned in the strategy. Furthermore, Cameroon is experiencing a steady decline in the oil sector that requires offsetting improvements in the non-oil sector. Without increased diversification, economic growth will continue to fluctuate considerably with the frequent terms of trade shocks. This would force the authorities to make frequent adjustments, thus compromising a sustained and consistent implementation of sector strategies and possibly worsening vulnerability and poverty. Therefore, the government is determined to accelerate the pace of reforms in order to diversify the economy and raise the growth rate to around 6 percent on average during the period from 2005 to 2015 . This economic diversification strategy rests on the following essential pillars. 


\section{- Rural Sector: Supporting private operators to foster production and securing population revenue and food security}

17. The GOC will continue its strategy of disengagement from direct production activities, including privatization of state-owned agro-business and commercial enterprises, while promoting policies that support production as well as the social sectors. Within the framework of the PRS, the government has developed an integrated rural sector strategy that adheres to these broad principles. The strategy aims to raise productivity and yields, thus fostering agricultural growth. It includes four priority areas, namely: (i) modernizing production equipment by facilitating medium and long-term financing and strengthening the human resource base through improved professional training, (ii) developing infrastructure in the rural sectors (roads, feeder roads, water, etc.), (iii) restructuring and strengthening the institutional framework and promoting an enabling environment for growth, and (iv) managing natural resources in a sustainable manner.

\section{- Manufacturing Industry: Promoting competitiveness and supporting the development of agro- industries, textile manufacturing, and wood processing to increase the contribution of industry to growth}

18. In the medium term, the industrial sector is regarded as the main engine of growth for sustainable poverty reduction. Reaching the target of 6-7 percent annual GDP growth rate to achieve the 2015 strategic objective will require a greater contribution from industry and services. In this area, Cameroon has a number of important competitive assets, including a privileged geographic position in the Gulf of Guinea with a potential market of 200 million consumers in Central Africa and Nigeria, as well good potential in important production factors such as energy and skilled labor.

19. However, Cameroon has not yet lived up to its potential in terms of industrial development, primarily because of past non-competitive policies. Structural reforms undertaken by the GOC to liberalize the market and spur competition have already improved the situation. In the context of the PRSP, the government plans to prepare an industrial strategy and has also launched two comprehensive studies on the competitiveness and the sources of growth, respectively, of Cameroon's economy. The studies' findings will help the authorities to refine the industrial strategy, including specific actions in support of subsectors with high growth potential. Meanwhile, the GOC's overall industrial redeployment strategy rests on the PRSP's key cross-cutting policies and remains consistent with the general principle of indirect and selective support, but without direct participation by the state in production and commercial activities.

20. Specific support programs could be designed to shore up promising subsectors based on the findings of the aforementioned studies. Preliminary study results indicate that a few sectors deserve special attention, including the textile/garment clusters as well as aviculture industries and the advanced wood processing sectors. They also indicate that agro-business, textile manufacturing, and tropical fruit and vegetable processing constitute good prospects for enhanced growth.

\section{- Services: Promoting tourism, information and communication technologies, transport, and finances}

21. Cameroon has important tourism assets which can be developed to increase value added, generate foreign exchange, and create opportunities for the poor, especially in handicraft and related services. In this context, a tourism sector strategy is being prepared, including several programs, namely: (i) promoting tourism activities, (ii) developing priority sites, (iii) drafting an Investment Code for Tourism, and (iv) undertaking studies for the integration of young graduates into the tourism sector. Implementing such action plans would also increase private investment in related activity sectors such as transport; financial services; agro-businesses; the hotel, food, and restaurant industries; agro-industry; and handicrafts. These activities are especially labor-intensive and thus constitute important vectors for pro-poor growth. 
22. Information and communication technologies also represent an important sector of activity that has seen significant growth recently and has the potential to become one of the major growth vectors for Cameroon in the medium term. Difficult access to, and lack of, information were mentioned during the participatory consultation process as key factors in isolation and poverty. In this area, the government will pursue a two-track strategy. The first track deals with direct promotion activities designed to foster sector development. To that effect, the government created a National Information Communication Technologies Agency (ANTIC, April 2002) to promote the development of information centers (cyber cafes) and to facilitate access by the population, including in rural areas. The government is also considering fiscal measures (such as reducing import tax and duties on computer equipment) for lowering costs and promoting competitiveness and growth. A second track addresses the population's access to information services. To this end, the government drafted an ambitious plan, including opening community multimedia centers in the provinces, to facilitate access to Internet services for landlocked and poor populations, so that they could benefit from valuable information about health, education, agriculture, livestock, and the environment. In a program that will receive financial support from UNDP and UNESCO, the government also intends to increase the number of rural radio stations beyond the 15 that are already operating. Lastly, the government will implement an important outreach program based on modern information technologies in order to shore up the fight against HIV/AIDS nationwide.

\section{Strategic Area 3: Strengthening the private sector as the main engine of growth}

23. The GOC will continue to directly support economic growth by allocating adequate public resources to priority sectors and providing targeted support to pro-poor growth sectors as indicated previously. However, the cornerstone of the government's strategy rests on a dynamic private sector, which would drive economic growth while efficiently distributing its fruits, contribute to a stronger domestic savings, and become an effective partner to foreign investors. Ongoing structural reforms, including price liberalization; the elimination of restrictive credit control practices; simplified tariff systems and customs procedures; the restructuring of the banking sector; privatization and port reforms; and the state's divestiture from productive activities have already helped create a more business-friendly and stimulating environment for the private sector.

24. The GOC is keen on keeping this momentum, and it has committed itself to deepening reforms in the following specific areas: (i) improving the physical environment of businesses by accelerating the development of transport, telecommunication, energy supply, and distribution infrastructures; (ii) improving the institutional and regulatory framework and delivering more efficient public services to businesses; (iii) enhancing security for investment by improving the judiciary system and implementing the new OHADA business legal framework; (iv) promoting Cameroonian products on external markets; and (v) promoting dialogue and partnership with private sector organizations.

25. In addition to this overall strategy, the government is determined to support and use the private sector, especially the SME/SMIs, as the prime instrument for wealth creation and social services development and delivery. The government's strategy for private sector development also has specific components targeting SMEs and micro-enterprises. The objectives are: (i) to reinforce private sector participation in capacity building, including supplying such social services as education and health; (ii) to enhance competitiveness, especially for SME/SMIs with high-growth potential; (iii) to diversify and adapt financial instruments and develop microfinance in order to mobilize financial resources for SME/SMIs and micro-enterprises; (iv) to foster greater organization and professionalism among small enterprises and the handicraft subsectors, for instance, promoting partnerships through the development of networks/clusters, micro-enterprises and business incubation; and (v) to improve the impact of the privatization program on the subcontracting of services to SME/SMIs. The government will also continue its policy of institutional support to and consolidation of the traditional financial sector as well as microfinance through the new subregional regulatory framework. 


\section{Strategic Area 4: Developing infrastructure to support the production and social sectors}

26. The GOC's infrastructure development policy is broad and concerns all key subsectors, including road, air, and sea transport as well as telecommunications, energy, and water. However, within the framework of the PRSP, the participatory consultations have revealed that roads, water, and electricity are the major concerns of the population with regard to infrastructure services. Consequently, the GOC is determined to give priority in infrastructure development to: (i) improving the road network, in both volume (network length) and quality, (ii) improving access to drinking water, (iii) extending coverage in electric power to remote rural areas, and (iv) taking immediate actions to correct current deficiencies in the overall power supply.

\section{○ Developing road infrastructure}

27. The key objective is to interconnect all regions of the country, to integrate the populationsincluding those in rural areas - into the economy, and to improve access to basic social services. At the same time, the strategy will favor labor-intensive techniques, as in road and feeder road maintenance and rehabilitation programs, to create new economic activities and employment opportunities, especially for the poor.

28. These broad objectives are translated into three main programs. The first is new investments to extend the road network. Extension would include the Trans-African road (Cameroon- Nigeria, Cameroon-CAR) in NEPAD, the CAEMC network, and improvements to national trunk roads (particularly the connection between the two major urban centers, Douala and Yaoundé, and all provincial capitals). The GOC road extension plan intends to consider the needs of the various regions as well as the subregional framework. The second leg concerns rehabilitation of deteriorated networks and periodic maintenance of the entire road network. Third is the development of appropriate and more efficient medium and long-term financing instruments for supporting road development, including enhanced private sector participation and improved private as well as public capacities to execute planned road development programs.

29. Learning from past experience, the government has decided to give special attention to road maintenance, particularly rural roads, in order to avoid past mistakes that led to severe deterioration of road assets. To this end, the government has developed a New Rural Road Maintenance Strategy (NSERR), which favors the use of deconcentrated structures in rural road maintenance as well as a greater participation by the beneficiary populations. The distinguishing features of this new approach are that it is primarily community based and decentralized and it favors labor-intensive activities conducive to effective poverty reduction.

\section{○ Improving access to drinking water}

30. During the participatory consultations, the population mentioned serious problems with village water engineering and access to drinking water. Large urban centers have adequate supplies of drinking water, although water quality remains an important concern. The rural world faces both a problem of adequate water supply as well as one of water quality. This partly stems from geological and ecological conditions in certain regions. But lack of adequate policies in the sector is also to blame. Reflecting this rural-urban gap, access to drinking water was estimated at 86.2 percent in urban areas against only 31.3 percent in rural areas in 2001 , with an average of 50 percent for the country as a whole.

31. To address this critical challenge, the government has implemented several sector reform programs, including privatization of Cameroon's national water supply company and elaboration of a "Rural Hydraulic II" program, which aims to enhance access to drinking water for all regions by 2025. In particular, the program seeks to increase the current drinking water coverage in rural areas from 
31,3 percent in 2001 to 75 percent by 2015 and also to make appropriate sanitation services available in all regions. The program constitutes an umbrella structure for several water supply rehabilitation and extension projects, including the construction of priority bore holes and water wells in vulnerable areas, especially in the northern part of the country.

32. For the medium term, a key objective of the PRS is to implement a drinking water production and distribution system in 113 secondary urban centers that host a large proportion of the semi-urban population. To this effect, an extensive program of bore holes and mini-water systems has already received financing from HPIC resources. During the 2003 fiscal year, which is considered a test period, the plan calls for acquiring mobile water plants to supply drinking water for emergency situations such as in cases of important disruption of the water system in urban centers and health structures, severe droughts, epidemics, civil and humanitarian problems, volcanic eruptions, and floods. In parallel, the authorities are also developing a drinking water supply program targeting schools and health centers across the country. This program will include urban and semi-urban sanitation measures for sewage treatment, extension of the primary water drain system, and construction of new purification systems.

\section{- Sustainably managing and protecting the environment}

33. Cameroon is especially endowed with unusually rich and diverse ecological, cultural, and anthropological systems. Nearly 90 percent of African ecosystems are present in Cameroon, and they are located in the Sahelian, Sudanian, forest, mountain, maritime and coastal areas. Cameroon's wildlife is also one of the richest and most diversified on the African continent. It counts about 409 mammal species, 183 reptile species, 849 bird species, and 190 amphibian species. And despite a rapid pace of exploitation in the recent past, Cameroon's forests still cover more than 22 million hectares, making it the second largest producer of forestry products in Africa behind the DRC and the first exporter of wood products from Africa. A sustainable management of these important natural resources will certainly contribute to enhancing growth in industries, exports, and tourism, thus generating foreign exchange while also significantly improving the livelihood of Cameroon's population.

34. However, major obstacles still remain. First, several important ecosystems are rapidly deteriorating because of unsustainable exploitation (poaching, over-grazing, bush fires, and itinerant agriculture). Similarly, because of intensive exploitation, Cameroon's forests have been losing 100,000 hectares per year over the last two decades. The government is keen on renewing efforts to stop the degradation and overexploitation of the country's natural resources. Consequently, it has prepared a Natural Resources Development and Protection Program, especially targeting Cameroon's fauna. In 2001 , under the first phase of the program, the GOC created a network of 6,650,000 hectares of protected areas for fauna covering about 14 percent of the national territory. The protected areas comprise 10 national parks, 6 fauna reserves, 1 fauna sanctuary, 3 zoological gardens, 35 protected hunting areas, and 10 community-managed protected hunting areas. Another component of the program includes targeted actions for rationalizing environmental management (e.g., the Emergency Action Plan and the Interministerial Environmental Committee), as well as a comprehensive strategy to implement the 1992 Rio Agenda 21.

35. The government is also committed to accelerating the implementation of the forestry sector reform agenda, which would ensure sustainable exploitation and increase value added in the subsector while preserving Cameroon's national heritage. The forestry reform program is supported by the donors and aims to: (i) promote sustainable exploitation while preserving ecological stability, (ii) support the emergence of a high-value-added wood processing industry, and (iii) foster community participation in conserving and managing this national forestry heritage. Key measures include: (i) a competitive bidding system for awarding concessions; (ii) transparent planning of new concessions; (iii) implementation of a forestry tax revenue mobilization program (PSRF); and (iv) supervision of forestry development plans in close partnership with two international NGOs for more effective control of logging activities and enforcement of forestry sector regulations. 
36. Another major objective of the forestry reform program is to enhance forest-based income opportunities for village communities, and thus improve the livelihood of the rural population living in the forest zones. In this context, the government plans to grant interested local communities priority rights to designated "community forests" and to remit quote-part of collected forestry tax revenues to local communities.

\section{○ Extending telecommunication services and power to rural areas}

37. For many years, the government has given priority to the development of the telecommunication and power sectors as pillars of its structural reform program. After an unsuccessful first trial, the government has started anew the privatization process for CAMTEL. An action plan has been prepared to this effect, in close consultation with the World Bank, and the process is expected to conclude within the next two years. Meanwhile, the government is committed to enhancing competition and strengthening the regulatory framework for fostering development; it has recently taken steps to shore up the capacities of the national regulatory agency (ART).

38. An important component of the program, which has a direct link to the social strategies, addresses the extension of phone services to rural areas. The program plans to create a public enterprise that would work closely with private fixed and mobile telephone companies in order to extend telephone services and derivatives to the rural populations.

39. As in case of the telephone sector, the energy sector reform program constitutes the main instrument for extending electricity to the population, including to remote rural areas. In order to ensure that the program benefit both economic operators and the population as a whole, the government is taking steps to strengthen the capacities of the regulatory agency (ARSEL) and to work closely with AES/SONEL, the main operator in the energy sector. Both are steps in implementing the investment program that was agreed upon as part of the privatization deal. At the same time, the government is contemplating different options for improving the country's capacity to generate electricity, including the construction of a new hydroelectric power plant.

\section{Strategic Area 5: Accelerating regional integration}

40. Cameroon is the main economic engine of CEMAC, a position that is further strengthened by the country's proximity to Nigeria and to the ECOWAS bloc. In this respect, Cameroon has considerable competitive assets, including political stability, rich endowments of natural resources (energy potential, arable land, etc.), human resources (a relatively high school enrollment rate and a welleducated labor force adequately concentrated in major urban areas), a solid and still improving infrastructure base (ports, roads, airports, etc.) as well as a head start in industrial capacities. The subregional market offers Cameroon good opportunities for the valorization of these assets, which would also help the country to compete more effectively in the global world market. Therefore, the GOC is committed to working in partnership with other countries of the subregion in order to enhance cooperation and accelerate integration in the context of CEMAC and ensure that the fruits of integration are equitably shared among member countries, thus providing the resources for attacking poverty in Cameroon

41. To this end, the government will provide leadership in favor of a general strategy of openregionalism and enhanced cooperation within the CEMAC framework, which would expand markets while improving the global competitiveness of the subregion relative to the rest of the world. In particular, Cameroon is committed to maintaining fiscal discipline and harmonizing tax policy in order to meet CEMAC convergence criteria and contribute to macroeconomic stability, an essential pillar of the fixed exchange rate and the common currency system. The government will also rekindle efforts to strengthen the common market in order to provide private operators a broader scale enhancing and competitive environment that is favorable to industrial development. The regional strategy also plans to strengthen and consolidate financial and interbank markets within a common regulatory framework 
to create an enabling environment for foreign investment, to strengthen cooperation in major physical infrastructure investment projects, and to accelerate integration of the infrastructure services markets. The government will also implement subregional agreements that are designed to facilitate the free movement of populations across borders, including enforcing existing rules of establishment and residency and facilitating related remittances. This would allow labor to cluster into high-growth areas such as in Cameroon's main urban centers while also ensuring that migrant workers could help home countries and communities benefit from the fruit of their efforts.

42. Defining actions have been carried out in each one of those areas. Under the stewardship of Cameroon's government, regional integration has recently acquired new momentum as evidenced by major progress in several areas. Regarding the common trade policy, Cameroon and its partners in CEMAC have reorganized the common external tariff's structure to reduce its maximum level from 30 percent to 20 percent and the number of product categories from 5 to $4(0,5,10,20)$. This is expected to lower the simple average tariff to about 12 to 15 percent. A new generation of reforms is currently being implemented to eliminate non-tariff barriers in order to facilitate intraregional as well as external trade, including simplifying administrative procedures and the transit system and removing road barriers. The common monetary policy has also seen a major development, including the progressive reduction of direct advances to the state (1/10 per year over the next few years) starting in 2003 , the creation of a regional market of government bonds and private stocks, and the implementation of a new currency exchange regulation, particularly the transfer of international operations management responsibilities from public administrations to commercial banks. In the financial sector, the government recently completed the restructuring of the financial system, including the single licensing rule that would allow banks to open agencies in any member country. The government will also contribute to the reform and modernization of the payments system (supported by the World Bank with IDA resources), as well as implementation of the subregional regulatory framework of microfinance adopted by COBAC.

43. Other aspects of regional cooperation have recently seen important developments as well. In the forestry sector, regional cooperation concerning the conservation and sustainable management of the Central African forest has been strengthened with a framework established through COFIMAC. Regarding road transport, the CEMAC road transport master plan as well as the NEPAD network is being implemented. Air transport has also seen important developments, and the regional framework is being used to accelerate the implementation of the Yamoussoukro agreements while Cameroon and its neighbors are working to open a regional airline.

44. In addition to expected growth spillover, the Government of Cameroon is working to ensure that these enhanced integration and cooperation activities will translate into tangible benefits for the poor. The microfinance legislation, especially, is expected to help protect household savings, particularly that of the poor. Similarly, trade facilitation within the CEMAC and improvement in the regional road network will reinforce cross-border exchanges, which typically benefits small and medium-sized operators. In addition, the poor will benefit more directly from targeted cooperation and integration actions relative to the labor market and human resources. For example, in the area of health, the government is using the regional framework to shore up the fight against contagious diseases, especially HIV/AIDS. In particular, the GOC is committed to cooperating with neighboring countries to ensure better protection of regional road and river regional transport corridors, including the CongoUbangui and the Lake Chad corridors. These activities are supported by the UNAIDS and AfDB programs. In the areas of facilitating the free movement of people and integrating the labor market, a soon-to-be-completed CEMAC passport project constitutes an important step.

\section{Priority 6: Building capacity and enhancing human resources}

45. The cornerstone of Cameroon's social strategy is capacity building and enhancing human resources. This priority is a direct response to the challenge of a growing and increasingly young population. It directly strengthens the social sphere but also human capital formation, therefore laying out strong foundations for medium and long-term growth. In this area, the government has prepared 
several sector strategies aiming to: (i) promote basic education for all, (ii) improve the general population's health, particularly mothers and children, (iii) improve living standards in urban areas, (iv) fight unemployment and strive to integrate the vulnerable population into the economy, (v) promote gender equality, and (vi) create an enabling environment for the protection and harmonious development of individuals and families.

\section{- Improving access to basic education and vocational and professional training}

46. The ECAM survey's results and participatory consultations have stressed the importance of education and professional training as a means for escaping poverty. Taking this into account, the government, in October 2000, adopted an education sector strategy aimed at accomplishing the following: (i) broadening educational access while eliminating disparities between boys and girls (100 percent accessibility in 2008 and a 100-percent completion rate in 2015); (ii) improving the quality of basic education; (iii) developing vocational, educational, and professional training; and (iv) improving management and governance throughout the entire education system. The government is determined to implement this strategy in partnership with the private sector and civil society.

47. The government is well aware that reaching universal enrollment will require not only additional resources but increased efficiency in the education system. Indeed, simulations by the government's technical services have shown that without major changes to the current system the 2015 Education For All objective will not be financially sustainable. Therefore, the government has taken important steps toward improving efficiency, including: (i) a policy decision to drastically reduce repetition rates by eliminating repetition within subcycles of the primary school system, (ii) a new policy regulating transition from primary to secondary education, and from lower secondary education to higher secondary education, and (iii) a new teacher recruitment policy. These policies will be supported by other actions designed to improve education management.

48. Also, and as part of the strategy to improve the efficiency of the education system, the government is determined to promote enhanced governance in the education sector. This includes increasing deconcentration and decentralization of resource management, enhancing participatory management, containing costs, and strengthening institutional capacities.

49. Vocational and professional training have become very important in the education system, due to labor market requirements and demands from young people for more professional skills to facilitate their integration into the labor market. In August 2002, the government created the Ministry of Vocational and Professional Training with a mandate to address the need of this subsector. A sector strategy is being prepared that will provide a framework for national vocational and professional training policies.

50. The government is committed to developing effective partnerships with the private sector, NGOs, and communities in order to implement the above strategies, particularly in primary education, technical education, and vocational and professional training. Another important goal of such partnerships is to create a stimulating regulatory framework that would facilitate private initiative in education, from the primary to the tertiary level, while maintaining quality standards and reducing costs.

\section{○ Improving health care}

51. Improving the population's health is a major economic and social development objective as well as a poverty reduction goal. The government plans to achieve this objective by implementing the health sector strategy adopted in October 2001. In particular, a national malaria control strategic plan has been adopted, with a global objective in line with the "Roll Back Malaria" initiative. The purpose is to reduce, by 2010 , malaria-related morbidity and mortality by half, particularly among the most vulnerable populations (children younger than 5 years old and pregnant women). 
52. The government is committed to deploying a maximum effort to implement its AIDS strategy for 2000-2005, adopted in September 2000. The strategy comprises seven major subprograms, including: (i) developing a communication plan involving the media; (ii) undertaking an outreach campaign geared towards youth in schools, universities, and those in non-school environments, including women, workers, and the rural population; (iii) promoting the use of male and female condoms among target populations; (iv) creating centers for prevention and anonymous voluntary screening in each health district; (v) extending the national program for reducing HIV transmission from mother to child; (vi) developing an effective partnership involving communities, associations, the public sector, and private and confessional groups to intensify the fight against AIDS; and (vii) developing medical care and counseling services for people living with AIDS (PPVS) as well as those orphaned by AIDS.

53. The government will also implement the 5-year action plan in the Extended Program for Immunization, incorporating the following priorities: (i) mobilizing additional resources for "immunization independence" and introducing new vaccines (against yellow fever and hepatitis B), (ii) training/retraining staff and rehabilitating/renewing equipment, (iii) developing communication materials to promote behavioral changes, (iv) outsourcing program implementation responsibilities to health districts, and (v) enhancing the program's monitoring and evaluation activities.

54. The government has also taken steps toward improving access to drugs, reactants, and medical supplies essential to the population. These measures have already resulted in a 40-percent lower drug price and greater availability in all health facilities. The government will pursue these efforts by implementing the national pharmaceutical master plan.

\section{- Carrying out a poverty reduction strategy in urban areas}

55. Cameroonian cities, particularly the major urban centers, are confronted with many difficulties: squatting, health hazards, lack of integration in certain neighborhoods, insecurity, unemployment, and a rise in serious crime. Furthermore, as mentioned above, nearly three- quarters of Cameroon's population are less than 30 years old. These young people tend to regroup in urban centers, creating additional pressure for social services, infrastructure, and labor market and adding to urban insecurity.

56. To meet this challenge, the government has prepared an integrated urban development strategy, aiming to (i) improve living standards among urban populations, a major portion of which lives in uncertainty, (ii) strengthen the economic role of towns through the extension, rehabilitation, and maintenance of urban infrastructures, and (iii) develop a social habitat promotion program in urban areas. A study has been initiated toward preparing a national housing policy. At the same time, the government has launched priority actions to correct the most urgent problems, particularly urban transport in Douala and Yaoundé, sanitation and waste management, and social care services targeting street children and mental patients, as well as an awareness campaign among prostitutes about the risks associated with sexually transmitted infections (STI) and HIV/SIDA.

\section{○ Undertaking other social development policies and strategies}

57. The participatory consultations highlighted that a dysfunctional social system in Cameroon, including social exclusions and discriminatory practices, is a major contributing factor to poverty. In response to these concerns, the government intends to finalize, in 2003, a social development strategy to step up the integration of vulnerable groups into the economy and society. In particular, the strategy would aim to create an institutional, legal, and regulatory framework guaranteeing rights and social protection to vulnerable groups. It also intends to (i) finalize the Family and Individual Act, (ii) finalize the draft law relative to the social protection of children, (iii) revise the law relative to the protection of handicapped persons, and (iv) draft a law regarding social integration of the population.

58. The government will also continue its effort to enforce women's rights, recognize their contribution to development, and foster their greater integration into economic activities. To this end, the government will prepare, before the end of 2003, a sector strategy to promote the role of women, 
with the following priorities: (i) improving the legal and social status of women, (ii) improving women's standard of living, (iii) promoting gender equality in every aspect of national life, and (iv) strengthening existing institutional structures and mechanisms to address women's specific problems.

59. The government is also committed to strengthening social security by reforming the system to ensure coverage of all social and professional categories left out by the current system. To that end, the GOC adopted a social security reform strategy document in 1998. Priorities of the strategy are: (i) extending social protection to informal sector operators, the rural population, professionals, traders, and self-employed workers; (ii) improving existing service delivery, including pensions, family care, and professional risks; and (iii) implementing new structures to foster more rigorous management, as well as legislation to enforce the reform.

\section{- Creating employment opportunities and integrating vulnerable groups into the economy}

60. The promotion of income-generating activities, including self-employment for the poor, constitutes a sustainable response to poverty. In its employment promotion strategy, currently in preparation, the government intends to (i) finalize and adopt a national employment policy declaration (PNE), (ii) create a National Employment Observatory, (iii) take appropriate measures to foster the development of SME/SMIs, and (iii) promote labor-intensive activities, particularly in road maintenance.

\section{Strategic Area 7: Improving governance, the efficiency of administrative services, and the institutional framework}

61. Promoting governance and curbing corruption are essential factors for a successful poverty reduction program. During the participatory consultations, the population ranked corruption and poor public management as important determinants of poverty in Cameroon. In the context of implementing the National Governance Program (PNG) adopted in June 2000, the government will emphasize: (i) reinforcing transparency and accountability, (ii) improving the delivery of basic social services, (iii) strengthening the rule of law and the legal and judicial security of investments, (iv) pursuing the decentralization and deconcentration of public management, and (v) improving citizen's access to information on public affairs.

62. The government has also launched major reforms toward improving the efficiency of financial management, particularly improving the quality of public expenditures and the absorptive capacity at the frontline. In particular, a vast budget reform will help track expenditures more efficiently and in a more transparent manner (SIGEFI). An important budget tracking exercise is also being implemented to track expenditures in the areas of education and health. Streamlining such an exercise into the budgeting system will provide the government with an important instrument to track expenditures and assess its efficiency, especially in priority areas, in order to design and implement appropriate reforms.

63. In the medium term, the fine-tuning of sector strategies and instruments will also help strengthen the budget process, facilitate the definition of priorities, and better target expenditures in alignment with the PRSP priorities. Finally, the government understands that the current year-to-year budget management practice is poorly adapted to the medium-term vision and new type of administrative management that the PRSP requires. Work is underway to explore the opportunity for preparing budget estimates to ensure greater consistency between the Medium-Term Expenditure Framework (MTEF) and PRSP priorities. These activities will help make the management of public expenditures more effective. These reforms will require targeted human and technical capacity building within the administration, particularly in the macroeconomic framework, sectoral medium-term expenditure framework, and budgetary planning.

64. Major reforms have also been initiated by the government for budgetary resource mobilization. In this context, the authorities are implementing programs to strengthen tax and customs administration. In particular, the government implemented a large-scale tax reform program aimed at 
modernizing the tax system, strengthening tax administration, and improving its productivity. The reforms have already translated into increased productivity and effectiveness in the system as a whole, as well as a new generation of programs, including the possible introduction of a property tax. This new generation of programs will help modernize the tax system and consolidate gains from past reforms. Similarly, the government has undertaken an important program toward modernizing and improving the efficiency of the customs system. These efforts will contribute to providing the government with the internal resources needed to finance its strategy.

\section{Ensuring Overall Financial Consistency, Costing, and Financing of the PRS}

65. The government carried out an important quantitative analysis to simulate the medium-term growth profiles, taking into account the sector strategies mentioned above. The simulation exercise projected the macroeconomic and financial accounts (GDP and its components, the government's financial operations, balance of payments, monetary situation, and debt). A medium-term expenditure framework (MTEF) was also prepared to indicate how budgetary resources need to be allocated to different sectors according to stated government priorities. In parallel, sector-level MTEFs have also been prepared, providing cost estimates of priority sector programs (education, health, and infrastructures). These programs have subsequently been revised downward in order to ensure consistency between the sector MTEFs and the overall macroeconomic framework.

66. Medium-term growth profile. Simulation results show that an effective implementation of the strategies would raise medium-term growth from an average of 5.5 percent for the period 2004-2007 to an average level of 7 percent between 2008 and 2015. This would mean a sustained average growth rate of 6 percent for the entire projection period. The higher growth rate is from increased contribution from the industrial sector (averaging 7.5 percent for the period) and services (approximately 7 percent for the period). At the same time, the overall investment rate is projected to rise from 18 percent in 2003 to roughly 24 percent during the $2007-2015$ period. In particular, private investment would come from markedly improved private saving, the result of a 3 percent annual per capita growth rate combined with a smaller increase in per capita consumption of about 2.5 percent per annum over the period.

67. Trends in poverty incidence. As a result of a higher per capita growth rate, the income poverty rate would converge toward the 20 to 25 percent MDG target by 2015, depending of the distribution assumption used. Therefore, in the central growth scenario, Cameroon would achieve one of its key millennium development objectives, namely halving income poverty by 2015 .

68. Medium-term expenditure framework (MTEF). The MTEF provides sector budget allocations over the 2004-2015 period. Accordingly, the priority sector shares of domestic resources (including current as well as investment budgets) increases in line with stated strategic objectives, from 3.2 percent to about 4 percent from 2003 to 2015 for education, from 1 percent to about 2 percent for health, from 0.2 to 0.4 percent for social development, and from 1.5 to 2 percent for productive infrastructures.

69. Alignment of priority financing needs with the MTEF. The costing of sector strategies reveals financing gaps between allocated resource envelopes and the financing needs of the main priority sectors. Aligning sector financing needs on MTEF allocations ("PRSP scenario") eliminates financing gaps, but this also implies a lower pace of execution of priority programs and related MDG/PRS objectives.

70. Cost of the strategy. Cameroon's poverty reduction strategy is comprehensive and integrated. Therefore, virtually all sectors are affected by the previously described strategic priorities, programs, and projects. In this context, the authorities have chosen to provide a full budget costing of the strategy, rather than "the marginal cost" of specific "poverty-reduction" expenditure programs. Accordingly, the total cost of the strategy would increase from CFA 950.5 billions in 2003 (that is, the total ministerial budget for FY 2003) to CFA 1,360 billions in 2007, averaging a 9 percent increase

xxii 
per annum. The subtotal for priority sectors would increase from CFA 568 billions in 2003 to CFA 866 billion in 2007, an 11-percent average annual growth rate. The share of the priority sector in the total budget would increase from 56 percent in 2003 to 64 percent in 2007, implying a net gain of 8 percentage points over the period. Priority expenditures could be increased without "crowding out" budget allocations to other sectors because of savings from debt relief. Hence the total budget (ministries and other budget items, including debt servicing) would increase by only 5 percent per annum over the period, which is 2.5 percent below the average nominal GDP growth rate. Domestic resources would cover about 92 percent of the budget, with the remaining 8 percent being covered by external resources.

71. Strategy financing. The government is determined to deploy renewed efforts to marshal external and internal assistance to finance the poverty reduction strategy. In particular, it will pursue the implementation of ongoing reforms to improve performance of the tax system (tax and customs). Similarly, it plans to work closely with its development partners to mobilize external resources and coordinate support to the PRS according to set priorities. Such a partnership will be organized through the consultation process initiated with the preparation of the PRSP, which will also be used for monitoring strategy implementation. Furthermore, the government will deploy efforts to create an enabling environment for the participation of the private sector in the domestic financing of the strategy.

72. Risk assessment. The PRSP's central scenario rests on key assumptions regarding the international environment, economic and social policies, and efficient execution of programs. A change in these assumptions could affect growth and hence the achievement of PRSP objectives. Key risk factors include: external shocks such as a decline in oil prices or a contraction in external resources flows, and persistent internal structural rigidities (for example, power shortages, unsatisfactory execution of the privatization programs, and low absorption of budgetary resources). Simulation results indicate that a decline in oil prices, continuing power problems, and weak mobilization or low absorption of programmed resources would trigger a decline in the average GDP growth rate ranging from 0.5 percent to 1.5 percent per annum according to the selected scenario, compared to the PRSP central scenario. The GOC is aware of such risks and is determined to take appropriate measures to mitigate the impacts of external shocks, protecting priority sectors as much as feasible. Meanwhile, the GOC is determined to accelerate and deepen reforms to reduce the endogenous risks mentioned above.

\section{Implementing and Monitoring the Strategy}

\section{$\bigcirc \quad$ Increasing participatory monitoring}

73. The government plans to step up participation in PRSP implementation, with the close association of all pertinent actors to the execution, monitoring, and evaluation of poverty reduction projects and the overall strategy. It will design approaches to foster the emergence of synergies by pulling together the capacities of all stakeholders, including beneficiaries.

\section{$\bigcirc$ Improving information system and monitoring indicators}

74. To ensure a successful implementation of the PRSP, the government plans to implement a poverty information system to strengthen and upgrade the existing statistical system. The new system would provide public agencies, the private sector, development partners, and civil society with timely information required to: (i) effectively manage poverty reduction activities and (ii) motivate participation by those concerned with such activities, as they would be better informed by a more transparent system. The system will be geared toward: (i) serving the needs of several poverty analysis objectives, (ii) providing timely readings of quantitative or qualitative indicators, permitting regular analysis, and (iii) enhancing the collection of data needed to produce such indicators. 


\section{Conclusion}

75. The success of Cameroon's poverty reduction strategy rests on the full support of and strong adherence by all social partners to the poverty reduction program. For that reason, it has been prepared through an extensive and sustained participatory process involving all stakeholders of Cameroon's society. It is expected that the strategy will evolve and be progressively fine-tuned as sector strategies become available and policies are implemented. The success of the strategy will depend on how effective the government's actions are, how well Cameroon's private sector rises to the challenge of competitiveness and growth, and how effectively civil society and communities are mobilized to take full ownership of the strategy. Success also rests on sustained and stronger support by all development partners. 
This page intentionally left blank 


\section{CHAPTER 1 : RECENT DEVELOPMENTS AND BACKGROUND TO}

THE PRSP

CInternational Monetary Fund. Not for Redistribution 


\subsection{INTRODUCTION}

76. Successive programs of macroeconomic stabilization and structural reform in recent years have succeeded in consolidating the government's fiscal position and improving the business climate, as well as the overall competitiveness of the Cameroonian economy. As a result, Cameroon has enjoyed annual economic growth rates of around 4.5 percent for the last five years, despite the continuing decline of the oil sector. Partly as a result of this solid macroeconomic performance, there has been a marked decline in poverty among the Cameroonian people. In particular, income per capita rose annually at about 2 percent over the period 1996-2001, accompanied by a significant fall of about 10 percentage points in the rate of monetary poverty over the same period.

77. Social conditions, however, have deteriorated considerably over the last two decades, as a result of the economic and social crisis, and growth has been neither deep enough nor durable enough to reverse these trends. Consequently, indicators of public health, education and access to basic services are still alarmingly low, and are in some cases worse than they were in the 1980 s.

78. In recognition of this situation, the government has committed itself to a new generation of economic and social reforms. Their objective is, on one hand, to consolidate the achievements of previous programs and stimulate economic growth, and on the other hand to address the social sphere and to ensure that solid economic performance translates into a real improvement in living conditions. To this end, the government prepared an Interim Poverty Reduction Strategy Paper (I-PRSP) in August 2000. Completion of the I-PRSP was followed by a series of sector strategy papers, focusing on priority sectors such as education, health and the rural sector, for which a social development strategy document is now being prepared. The present Poverty Reduction Strategy Paper (PRSP) is intended to bring shape and coherence to this new generation of economic and social policies, and to provide a frame of reference for Sustainable Human Development in Cameroon.

\subsection{THE MACROECONOMIC SITUATION}

79. Until 1985, following more than two decades of steady growth, Cameroon occupied an enviable position among the sub-Saharan African economies. The continued growth of agricultural output and exports was complemented in the second half of the 1970s by a start at exploiting the country's petroleum reserves As a result, Cameroon recorded average real growth rates in the order of seven percent over a period of some ten years.

80. During the budget year 1985/86, however, export revenues declined sharply. This collapse involved both oil and other exports, and export proceeds dropped by CFAF 329 billion, or 8.2 percent of GDP. The retreat in economic activity accelerated in $1986 / 87$, as a result of steadily falling prices for the country's principal exports (oil, coffee, cocoa, and cotton). Growth rates turned negative, and between 1985 and 1988 the terms of trade deteriorated by half.

81. To address the crisis, the government first adopted a domestic adjustment policy, which quickly revealed its limitations. The effect on the economy of government austerity and a reduced public sector was not enough to root out the deep-seated malaise. Economic indicators continued to deteriorate and the steady decline in incomes led to a 40 percent fall in per capita consumption between 1985/86 and 1992/93. The external debt stock rose from less 
than one-third to more than three-quarters of GDP between 1984/85 and 1992/93. Investment declined from 27 percent to less than 11 percent of GDP. To deal with the pronounced deterioration in government finances, marked by unsustainable cash pressures, civil service wages were drastically cut in 1993.

82. Beginning in 1994, new economic policies based on monetary adjustment, and the resulting competitiveness gains, succeeded in curbing this trend. Indeed, significant improvements were initially recorded in the export sectors and in the fiscal area. Nevertheless, the government's cash flow was still very inadequate and was not even enough to meet current expenses. Domestic and external debt servicing alike were at risk. Mounting payments arrears on the external debt made the country's relations with its partners increasingly difficult.

83. Two new structural adjustment programs concluded in 1994 and 1995 under stand-by arrangements with the IMF ended, like their predecessors, in failure. In particular, the objectives of restoring fiscal balance and servicing the external debt could not be met.

84. In August 1997, after satisfactorily completing a staff-monitored IMF program for the 1996/97 budget year, the government was able, for the very first time, to enter into an Enhanced Structural Adjustment Facility (ESAF) arrangement. The International Monetary Fund thereby threw its support behind the medium-term economic and financial program put in place by the Cameroonian authorities to cover the period from July 1, 1997 to June 30, 2000.

85. Execution of the staff-monitored program in 1996/97 was reinforced by a determined effort to restore Cameroon's external credibility, particularly by clearing external arrears of some US\$500 million. Furthermore, the government involved the private sector and civil society closely in drawing up the three-year economic and financial program, and in the negotiations with the IMF for the August 1997 arrangement. Thanks to this approach, economic operators and civil society were more willing to accept the necessary adjustments and reforms.

86. The government's economic and financial program for the period July 1, 1997 to June 30, 2000 has been satisfactorily implemented, with the decisive support of the international financial community, more specifically the IMF under the ESAF, now the PRGF, the World Bank, with its third Structural Adjustment Loan (SAL), the European Union, the African Development Bank, the Paris Club of bilateral creditors, bilateral creditors not participating in the Paris Club (Saudi Arabia, Kuwait, China) and France, with structural adjustment loans in addition to the debt relief granted under the Paris Club agreement of October 1997.

87. Despite the Naples-terms rescheduling agreement concluded in the fall of 1997 with the Paris Club, external debt service remained a heavy burden on the government's finances, draining away the resources needed by the government to fulfill its essential tasks (i) in fulfilling its sovereign duties (justice, security); (ii) in the social sectors (education and health); and (iii) in maintenance, rehabilitation and upgrading of infrastructure.

88. Cameroon's eligibility for the Enhanced Heavily Indebted Poor Countries Initiative (HIPC), declared by the IMF and World Bank Boards in May 2000, thus opened new prospects for the country, thanks to the budgetary savings that the country would enjoy following the decision point (which Cameroon reached in October 2000). 
89. The solid performance in implementing the three-year economic and financial program 1997-2000 produced a noticeable improvement in macroeconomic stability and laid the basis for sustained economic growth. From 1996/1997 to 1999/2000, annual real GDP growth averaged 4.5 percent, while inflation, measured by the consumer price index, was held to less than 1 percent. The current account deficit excluding official transfers declined from 3 percent of GDP in 1997/1998 to 1.5 percent in 1999/2000, as the terms of trade improved. The government finances improved considerably, thanks to greater mobilization of State revenues and a determined effort at expenditure control.

\subsection{THE SOCIOECONOMIC SITUATION}

90. The improved macroeconomic performance has not been accompanied by a comparable improvement in living conditions. It is true that economic growth has translated into higher incomes per capita (which rose by about 2 percent a year between 1996 and 2001), and a significant drop of 13 percent in the poverty rate, according to a comparison of results of the ECAM I and II household surveys.

91. Yet it is clear that social conditions deteriorated considerably during the years of crisis, and that economic recovery has done little so far to improve them. In particular, the provision of basic social services was severely affected by the government's financial difficulties during those years. The county's road infrastructure has deteriorated for want of maintenance. New road building has been halted. Water engineering and electrification programs, particularly in rural areas, have lagged for lack of financing.

92. The employment situation is of particular concern. The restructuring of public and parapublic enterprises, which entailed the closing of some establishments, a civil service hiring freeze, and labor-shedding measures, led to a significant rise in unemployment. Between 1984 and 1991, employment fell by 10 percent and the unemployment rate reached 17 percent in 1995. Joblessness has affected mainly young people and women, sparking a major expansion of the informal sector. In 2001, for example, roughly 8 percent of the labor force was unemployed: in rural areas, that rate was 2.3 percent, while in the cities it stood at 18.9 percent, reaching a peak in the cities of Douala (25.6 percent) and Yaoundé (21.5 percent). Yet this decline in unemployment is a result primarily of jobs created in the service sector, particularly in the informal economy, where job insecurity remains high. Unemployment is still high among young people, moreover, and is exacerbated by the mismatch between vocational training and the employment needs of the economy.

93. To address the situation, the government launched a series of measures through the National Employment Fund (FNE) designed to: (i) enhance transparency and intermediation in the labor market, (ii) provide vocational adjustment or on-the-job training to the unemployed to help them join the labor force, and (iii) support initiatives for the creation of individual microprojects. The government has also instituted a National Employment and Vocational Training Observatory (ONEFOP) to improve its understanding of the labor market. Other steps have been taken to reduce unemployment, and such as the ongoing dialogue between employers' organizations and the school and university system, and the consultations that the government has instituted through the Interministerial Committee, which has been extended to include the private sector. 
94. In the education sector, budgetary allocations declined sharply over the period, resulting in: (i) a shortage of physical facilities because of the halt to classroom instruction; (ii) a worsening of the pupil/teacher ratio, reflecting the teacher hiring freeze; (iii) shortages of teaching materials and other school supplies. Problems in the education system also reflect other ills, in particular (iv) a lack of equity and ineffective management of the system.

95. Technical education and vocational training. The onset of the crisis sparked a flood of applicants for technical education and vocational training, yet the lack of credit has made it impossible to develop the necessary facilities. Training programs are still inappropriate in light of socioeconomic and vocational realities, and teaching equipment is inadequate and obsolete, meaning that training is more theoretical than practical. As to vocational education, it remains in its infancy, and is still unstructured.

96. These factors have combined to produce a sharp decline in the quality of the education system, as reflected in: (i) high repetition and dropout rates; (ii) geographic and gender disparities in access to schools; (iii) disparities in the installation of school infrastructure; (iv) a deterioration in the quality of teaching and learning; (v) a high degree of centralization and inefficiencies in the management of the education system.

97. Higher education has suffered a similar fate, made worse by capacity problems in the face of a rapidly growing student body. When it was opened in 1962, the Federal University of Cameroon had about 600 students. That number rose to 7000 in 1970, 32,000 in 1990, 50,000 in 1992, and more than 71,000 in 2002. This sharp growth in demand for higher education has led to over-enrollment in the former University of Yaoundé, and the other four universities, at Buéa, Douala, Dschang and Ngaoundéré, have been unable to absorb the overflow.

98. As a result, higher education conditions and performance are deteriorating, with: (i) a worsening of the student/teacher ratio, (ii) inadequate guidance services for students, (iii) lower quality of teaching, and (iv) poor performance by the system, open internally and externally, as illustrated by high failure rates, in the professional faculties especially, and a growing number of graduates who cannot find jobs. There is also an imbalance in the allocation of the university budget, which is tilted towards administrative expenses to the detriment of teaching and research. This has worsened working conditions, and is demoralizing the university community.

99. In the health field, the problems are virtually the same as those in education. Budgetary cutbacks have led to: (i) a halt in the construction and equipping of health facilities; (ii) a freeze on the hiring of public health workers; and (iii) a shortage of personnel in both quantitative and qualitative terms. Moreover, the distribution of health workers across the country is inappropriate, and salary cutbacks for government employees have undermined their performance. As a result, the main quality indicators have deteriorated against WHO standards: there is one physician for every 10,000 inhabitants (compared to 1 for every 3000) and one nurse for every 2,250 inhabitants (compared to 1 for every 1000).

100. The collapse of the health system has come just as new challenges to the health sector have appeared. In particular, (i) the incidence of HIV/AIDS rose from 2 percent to 11.8 percent between 1992 and 2002, and is particularly severe among the working-age population,(ii) tuberculosis has returned with renewed intensity, generally in conjunction with HIV/AIDS, and (iii) malaria is persistent, accounting for between 40 and 50 percent of 
medical visits and 28 percent of hospitalizations. This situation is undermining the health of the Cameroonian people and reducing both the quantity and quality of human capital, which could have a severe impact on the outlook for economic growth over the medium and longer terms. Moreover, life expectancy at birth remains low (59 years) and infant mortality is still high (77 per 1000), as is maternal mortality (430 per 100,000 live births).

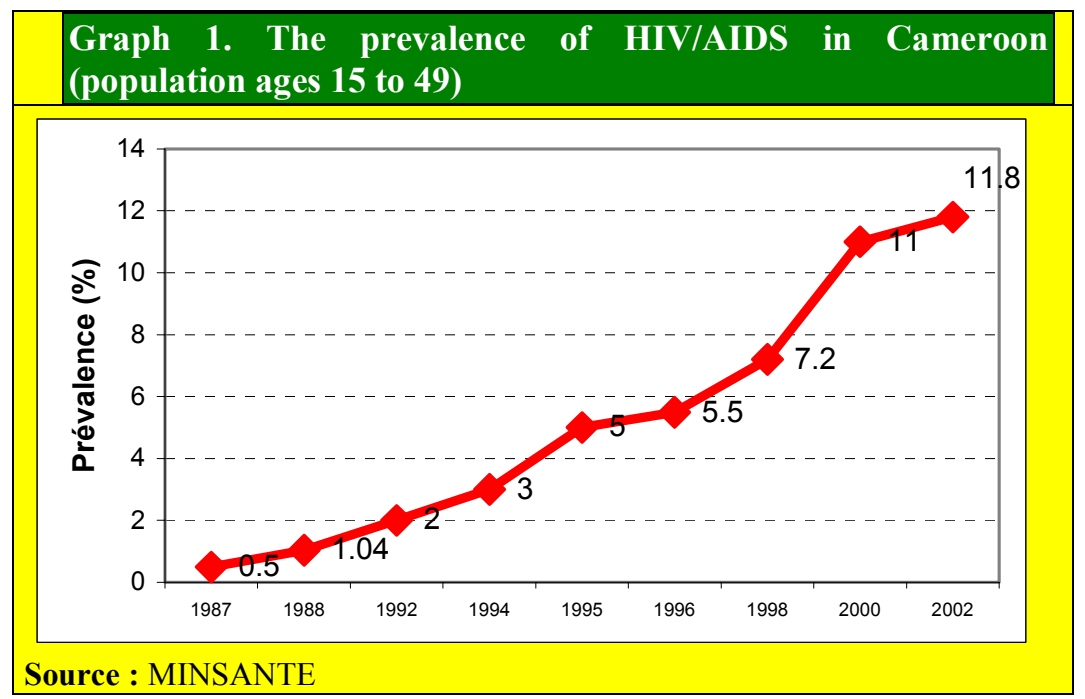

101. At the same time, Cameroon has been experiencing rapid and continuous urbanization, primarily through rural exodus. According to available statistics, nearly half of the country's population now lives in the cities. The average growth rate for the overall population is about 2.8 percent, but it is 5 percent in urban areas, reaching 7 percent in Yaoundé and 6.4 percent in Douala. Projections point to a doubling of the population of these two cities by 2015 . Under the combined impact of uncontrolled urbanization and economic crises, Cameroonian cities, particularly the larger ones, are confronted by many problems including the proliferation of squatter settlements, unhealthful and insecure living conditions, rising unemployment, and growing numbers of homeless people, street children, and the mentally ill.

\subsection{THE CONTEXT AND FEATURES OF CAMEROON'S PRSP}

102. The Cameroonian government is fully aware of the scope of these social problems and the need for a comprehensive medium-term development framework for coordinating all of its economic and social strategies. Such a framework would help to articulate an integrated vision of sustainable human development with a forward-looking set of sectoral strategies and programs for making that vision a reality. The government took some important decisions in this respect in the form of its Poverty Strategy Declaration, adopted in December 1998. The government has also subscribed to the Millennium Development Goals (MDG, September 2000), whereby 191 countries, including Cameroon, adopted a minimum set of targets for poverty reduction and human development by the year $2015 .^{1}$

\footnotetext{
${ }^{1}$ At the Millennium Summit in New York in September 2000, 191 countries, including Cameroon, represented by its Head of State, adopted the Millennium Declaration. It covered the major concerns of the planet, including peace, security and development, and called for reducing poverty, improving health and education, and promoting rights and freedoms, as well as environmental sustainability and the sound management of public affairs. A minimum set of 8 Millennium Development Goals (MDG) was established, and signatories undertook to achieve them by 2015 .
} 
103. A first important milestone in translating this vision into an action plan came in August 2000, when the government prepared its Interim Poverty Reduction Strategy Paper (IPRSP). The actions called for in the interim PRSP have been satisfactorily pursued, consistent with current programs, and the government has placed particular stress on the emergency measures described in that paper with respect to poverty, education, health and HIV, improving road maintenance and supporting SMEs, verifying and discharging the domestic debt, improving governance, and combating corruption. These initiatives have continued in other sectors, and the authorities drew upon this experience in preparing the PRSP. Since then, major efforts have been made to develop sectoral strategies. Strategy papers have now been prepared for national education, health and rural development.

104. The present Poverty Reduction Strategy Paper (PRSP) represents the culmination of these efforts. The PRSP is intended to be an empirical expression of a comprehensive framework for sustainable human development for Cameroon over the medium term, and to chart the course for the country towards the MDG. Although it is still a work in progress, this first version of the PRSP already contains a number of notable features and functionalities that the government intends to build upon, through an adaptive and participatory process.

105. A comprehensive development framework. In substance, the PRSP offers an empirical and integrated framework for the development of Cameroon, one in which macroeconomic, sectoral and social policies work together to promote growth, reduce poverty, and improve other dimensions of human development (education, health, security, etc.). In particular, the PRSP brings the government's macroeconomic and structural reform programs (PRGF and SAL III) into line with its sectoral strategies (education, health, infrastructure, etc.), thereby ensuring that these various pillars of economic and social policy will be mutually reinforcing over the medium term. The government has already adopted a full-scale action program for progressively refining the strategy through the development of other sectoral strategies, in the social area, infrastructure, industry and the urban sector.

106. A coherent financial framework. The PRSP also constitutes a coherent short- and medium-term financial framework for the government. It makes the trend of growth-generated revenues compatible with the financing needs of the sector strategies, while at the same time ensuring that the pace of execution of macro and sector strategies will have an impact on the economic growth path. Similarly, the PRSP offers a framework for bringing government fiscal and budgetary policies into line with the declared objectives of supporting the productive sector and the social sectors. It is the rigorous respect of macroeconomic, budgetary and sectoral frameworks that will ensure the short- and medium-term financial coherence of the PRSP.

107. A framework for coordinating government efforts and external support. The PRSP will be able to achieve its objectives only if sufficient political will and public resources can be effectively mobilized and targeted at the strategic issues indicated in the paper, and if the concomitant programs are properly executed. Similarly, in the case of the sectoral growth "forecasts", their "probability" will depend on the effective implementation of reforms. The PRSP offers a frame of reference for targeting and coordinating government action. It also outlines the place of the various assistance programs offered by the country's development partners. In this sense, it constitutes a framework for mobilizing and coordinating external assistance. The same is true of the government's macroeconomic program, which the IMF is 
supporting in the context of the PRGF, and the World Bank's Structural Adjustment Loan (SAL), for which the PRSP will now serve as the focal point. ${ }^{2}$

108. A framework for consultation and consensus-building with civil society, the private sector and development partners. The PRSP is the result of an intensive process of participation and consultation. The government organized participatory consultations throughout the country in order to enlist the public and civil society in identifying economic and social problems and in formulating the strategy. The government intends to institutionalize this approach for monitoring and periodic review of the strategy. In the same spirit, the government instituted a process of ongoing consultations with development partners for preparing the strategy. It intends to reinforce this process for implementing, monitoring and reviewing the PRSP, and for mobilizing and coordinating external assistance.

109. Underlying the preparation of the PRSP was a series of wide-ranging analytical studies designed to clarify choices, identify priorities, and ensure compatibility between ends and means. In particular, and with the support of the World Bank and the European Union, the government conducted large-scale statistical studies to assess the scope of poverty in Cameroon (ECAM I and II), and to analyze its dynamics and its determining factors as the basis for designing a poverty strategy. A schedule of in-depth analytical studies was prepared for conducting this work in the context of monitoring the strategy and making future revisions to it. With support from the UNDP and the World Bank, the government has also developed an instrument for macroeconomic and budgetary scaling, so as to simulate the growth profile and ensure consistency between the macroeconomic framework and the sectoral strategies of the PRSP. At the same time, sectoral analysis models have been developed for quantifying sectoral strategies and placing them in relation to the macro framework. The government intends to pursue these modeling exercises and to reinforce its technical capacity to monitor the program and the strategy.

110. The government, again with the support of the UNDP and the World Bank, has launched a "Comprehensive economic study on the sources of growth for reducing poverty", 3 to identify competitive industries and recommend support policies. At the same time, the authorities have undertaken a large-scale study on the competitiveness of the Cameroonian economy ${ }^{4}$, an interim report on which is now available. The initial results of these studies were used in formulating the PRSP, while the final results will provide input to the next revision of the paper. Finally, in order to have the strategy reflected regional specificities, a series of socioeconomic studies ${ }^{5}$ has been initiated with UNDP support. These studies should provide an effective instrument for monitoring the execution of the strategy and its impact in the regions, from the viewpoint of effective decentralization.

\footnotetext{
${ }^{2}$ In the context of the PRSP, the World Bank has developed a new instrument for channeling its support, the Poverty Reduction Support Credit (PRSC).

${ }^{3}$ See "Etudes sur les Sources de la Croissance pour la Réduction de la Pauvreté au Cameroun" (Study on the Sources of Growth for Reducing Poverty in Cameroon), BDS, 2002.

${ }^{4}$ See "Etude Documentaire sur la Compétivité de l'Economie Camerounaise" (Documented Study on the Competitiveness of the Cameroonian Economy), Competitiveness Committee, Interim Report, Yaoundé, March 2003.

${ }^{5}$ See report on “Etudes Socioéconomiques du Cameroun”, UNDP, Yaoundé, 2002.
} 


\section{CHAPTER 2 : THE CHARACTERISTICS OF POVERTY IN}

CAMEROON

CInternational Monetary Fund. Not for Redistribution 


\subsection{DEFINITIONS AND PERCEPTIONS OF POVERTY}

111. Assessing the poverty status of a person or group of individuals is a complex undertaking. The concept of poverty is essentially a relative one. Within any human group, the poor are, schematically, that portion of the population whose living conditions are manifestly below the observed average. This allows us to identify a kind of demarcation line between the "poor" and the "nonpoor", one that varies in space and in time. In Cameroon, it is now possible to identify the "poor" population using a variety of sources and a broad range of characteristics that are often closely linked to the sociocultural setting.

112. As part of the participatory process for preparing the PRSP, one of the first concerns of those involved was to ensure that they had properly identified the scourge they were seeking to combat, i.e. what it was that made people "poor". As a prelude to the participatory consultations with grass-roots communities, a seminar was held to launch the PRSP preparation process in January 2000, at which participants defined poverty in terms of material deprivation, food insecurity, lack of access to social services (health, education and basic training), decent employment, safe drinking water, social protection, reliable information, housing, transportation, and the lack of involvement in decision-making.

113. The participatory consultations for preparing the PRSP found that nearly all the people interviewed in the field saw poverty as above all the lack of material or financial resources to satisfy their basic needs. These needs include food, housing, health care, education, safe drinking water, etc. The lack of services for meeting these basic needs in some localities can also make people poor, even in the case of families who have the means to pay for them.

114. In its 1998 Human Development Report on Poverty in Cameroon, the UNDP considers poverty as a complex phenomenon that generally refers to inadequacy of resources and deprivation of choices that would enable people to enjoy decent living conditions. Thus poverty has a multiplicity of dimensions, including poor health and education conditions, lack of access to knowledge, the impossibility of exercising civil rights, lack of dignity and personal confidence, degradation of the environment, etc.

\subsection{THE QUANTITATIVE OR MONETARY APPROACH TO POVERTY}

\subsubsection{Household living conditions surveys}

115. The quantitative or monetary approach to poverty is based on the method of basic food and nonfood needs, identified using data from the two Cameroonian Household Surveys (ECAM I and II) that were conducted nationwide in 1996 and 2001. They provided a clear picture of the status of poverty and living conditions in Cameroonian households.

116. The 1996 household survey (ECAM I), which was the first of a series, was conducted just as Cameroon was emerging from a severe economic crisis that had lasted for nearly a decade. That survey was able to measure the effects of the crisis and of structural adjustment programs on household living standards and conditions. The ECAM II survey, which was undertaken in September 2001, updated the poverty profile and served in preparing benchmark indicators to monitor progress in reducing poverty. It also provided the basis for preparing the complete PRSP. 


\section{Box 1. Standard of living and poverty threshold indicator}

Annual household consumption is the best reflection of living standards relative to income, but it is very difficult to measure. Final household consumption as constructed for this purpose includes, in our context, four distinct elements, namely: monetary consumption, self-consumption, transfers in-kind from other households, and imputed rent.

Self-consumption and transfers in-kind were valued at the time of data collection. Self-consumption was corrected for under-estimation using data collected during the survey on unsold agricultural production. Durable goods were recorded at 5 percent of their user value. Health expenditures considered in the indicator referred to medical consultations. Expenses for hospitalization, medical evacuation and physical rehabilitation devices were excluded.

The benchmark poverty threshold used (based on the basic needs approach) was calculated using a food threshold, to which was added an amount for nonfood basic needs. The food threshold was calculated from a household food basket composed of 61 goods representative of consumer choices as revealed by the survey. Purchasing power parity indices for Yaoundé and other regions were calculated so as to value the basket of goods at Yaoundé prices. For example, an index of 0.950 for a region means that a basket of goods that would cost 1000 CFA francs in Yaoundé will cost 950 CFA francs in this region.

The goods selected were combined to provide a working-age adult with a daily dietary consumption of 2900 calories. This caloric level was selected in light of the adult equivalent scale ("Recommended Dietary Allowance", RDA) used for standardizing household expenditures. It is constructed on the assumption that an adult consumes 2900 calories per day, depending on age and sex. On this basis, the food threshold calculated at Yaoundé prices is 151,398 CFA francs per year and per adult equivalent.

For determining the total threshold, the nonfood portion is estimated as a fraction of the food component. The minimum threshold was set at 232,547 CFA francs, and the maximum threshold at 345,535 CFA francs per year and per adult equivalent. Thus, households where the annual consumption expenditure per adult equivalent is clearly below the minimum threshold are considered "poor". Those whose expenditure falls between the minimum and maximum thresholds are considered as "intermediate", and households whose expenditure exceeds the maximum threshold are considered "nonpoor".

Source : MINEPAT/DSCN

117. The ECAM II survey covered all 10 provinces of Cameroon, and was conducted in both urban and rural areas using a sample of 12,000 households, of which 10,992 were actually visited. By contrast, for the ECAM I survey the country was divided into six strata (Douala, Yaoundé, other cities, forests, high plateaus, savanna) and the sample size was 1800 households, of which 1731 were actually interviewed.

\subsubsection{The standard-of-living indicator and the poverty threshold for 2001}

118. The standard of living indicator used for determining the poverty threshold is annual household final consumption. The poverty threshold was thus set at 232,547 CFA francs in 2001 , versus 148,000 in 1996 . The corresponding incidence of poverty is 40.2 percent for 2001, and 50.5 percent for 1996 . For purposes of comparing the poverty situation between 1996 and 2001, a new threshold of 185,490 CFA francs was estimated. The incidence of poverty was then reevaluated at 53.3 percent in 1996; the indicators for 2001 remained unchanged, ECAM II being taken as the benchmark survey, because of the size of its sample. Most of the ECAM survey data used in this paper for analyzing this dimension of poverty were calculated for comparative purposes. 


\subsubsection{The changing poverty profile in Cameroon}

\subsubsection{Incidence and intensity of poverty}

119. On the basis of the two surveys, ECAM I and II, the poverty rate declined by about thirteen percentage points over the five years, from 53.3 percent to 40.2 percent of the population. ${ }^{6}$ During the same period, the gap by which the average income of poor households fell below the poverty line also improved, shrinking from 19.1 percent in 1996 to 14.1 percent in 2001 . This indicates that in 1996 it required an average annual supplementary transfer of 35,426 CFA francs to lift an individual out of poverty, compared with 26,154 francs in 2001 .
Table 1 : Poverty rate trend $(\%)$

\begin{tabular}{lccc|} 
& 1996 & $\mathbf{2 0 0 1}$ & $\begin{array}{c}\text { Change } \\
\text { As\% }\end{array}$ \\
\hline Incidence (P0) & & & \\
Rural & $\mathbf{5 9 , 6}$ & $\mathbf{4 9 , 9}$ & $-\mathbf{9 , 7}$ \\
Urban & $\mathbf{4 1 , 4}$ & $\mathbf{2 2 , 1}$ & $\mathbf{- 1 9 , 3}$ \\
Total & $\mathbf{5 3 , 3}$ & $\mathbf{4 0 , 2}$ & $\mathbf{- 1 3 , 1}$ \\
Depth (P1) & & & \\
Rural & $\mathbf{2 1 , 5}$ & $\mathbf{1 8 , 3}$ & $\mathbf{- 3 , 2}$ \\
Urban & $\mathbf{1 4 , 7}$ & $\mathbf{6 , 3}$ & $\mathbf{- 8 , 2}$ \\
Total & $\mathbf{1 9 , 1}$ & $\mathbf{1 4 , 1}$ & $\mathbf{- 5 , 0}$ \\
\hline Source_: DSCN, ECAM I and II Reports & \\
\end{tabular}

\section{Box 2. Heavy inroads against poverty}

The poverty assessment study was conducted on the basis of comparability-adjusted data from ECAM I of 1996 and ECAM II of 2001. The main difficulty was to construct comparable aggregates between the two years, given the differences in the questionnaires, the survey methodologies, and the sampling schemes. After careful examination of these differences, a number of correction factors were considered. With these correction factors, 12 welfare measurement indicators were calculated and the sensitivity of the results was analyzed.

A sensitivity analysis of dynamic comparisons (national, urban, rural) and inter-regional comparisons (urban vs. rural) was performed for these 12 indicators. The results show that, regardless of the level of the welfare indicator used, poverty declined between 1996 and 2001.

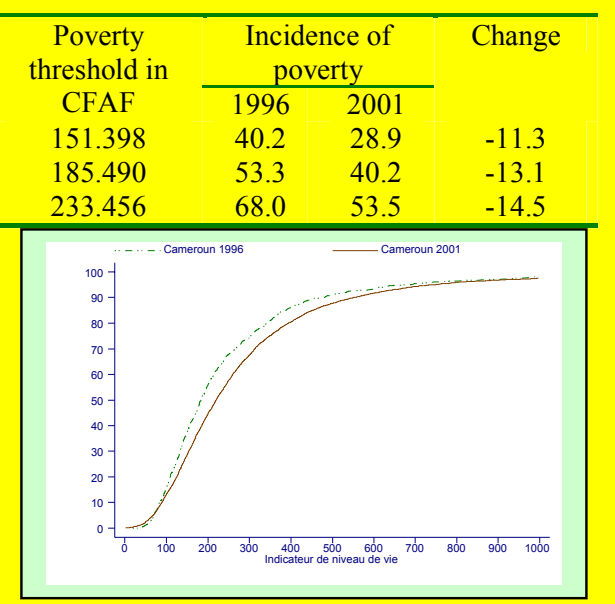

In arriving at this conclusion, we conducted a dominance analysis to confirm that poverty had indeed retreated between 1996 and 2001, regardless of the poverty threshold selected. This analysis involved examining the household income distribution functions for 1996 and 2001, plotted on the same graph. For each point on the curves, the abscissa (horizontal or $\mathrm{x}$-axis) represents a certain income level, while the ordinate (vertical or $\mathrm{y}$ axis) represents the proportion of individuals with incomes below that level. By setting the poverty threshold at any point along the x-axis, we can find the poverty incidence (the ordinate) corresponding to that threshold.

The distribution functions show that, with a "reasonable" poverty threshold, the 2001 income distribution dominates that for 1996. In other words, whatever the poverty threshold selected as reasonable, poverty is consistently lower in 2001 than in 1996. The decline in poverty between 1996 and 2001 is therefore a robust result that is not dependent on the poverty threshold selected. The above table makes clear this decline in poverty between the two years, as illustrated by one of the 12 welfare indicators and three different levels for the poverty threshold.

[Graph: x-axis: "Standard-of-living indicator"]

Source : MINEPAT/DSCN

\footnotetext{
${ }^{6}$ Further calculations were needed to make the two surveys comparable: in particular, the two surveys had to be harmonized. Using a "standard basket," the poverty threshold was reevaluated at 185,490 CFA francs per adult equivalent, per year
} 


\subsubsection{The regional dimension of poverty}

120. The survey results also show that poverty is still more pronounced in rural than in urban areas. In 2001, eight poor people out of ten were living in the countryside, and the incidence of poverty there is more than double the incidence in the cities. An analysis of the depth of poverty shows that it would take an income transfer of some 43,500 CFA francs per year to lift a rural dweller out of poverty, or four times as much as it would take for an urban dweller (10,000 CFA francs). The two largest cities, Douala and Yaoundé, account for 20 percent of the country's total population, but only

\begin{tabular}{|lccc|}
\hline Table 2 : Incidence of poverty by region \\
(\%)
\end{tabular}
11.8 percent of those classed as poor.

121. The analysis of rural poverty shows that it is distributed according to agro-ecological zones. Incidence is highest in the forest zone and in the high plateaus. In those areas, however, there has been a noticeable decline in poverty: the incidence was 55.4 percent and 50.7 percent, respectively, in 2001, compared to 72.5 percent and 62.9 percent in 1996, representing a decline of 17.1 and 12.2 percentage points respectively. On the contrary, the phenomenon has accentuated in the savanna zone (especially in the North and Extreme-North provinces), where the incidence rose by 1.3 percentage points.

122. The situation described above, which is common to many African countries south of the Sahara, suggests that policies and strategies for reducing poverty should place particular emphasis on the countryside, and on a region-by-region approach. 


\section{Box 3. The decline of poverty : the growth effect and the redistribution effect}

A comparative analysis of results from the ECAM I and II surveys suggests that the incidence of monetary poverty declined by about 13.1 percentage points between 1996 and 2001 , from 53.3 percent to 40.2 percent. This decline may have resulted from strong growth in average incomes (the "growth" effect), or from an improvement in income distribution (the "distribution" effect).

A breakdown of the poverty trend in Cameroon shows that its decline is due primarily to the effects of economic growth, rather than to redistribution. Of the 13.1 percentage points by which poverty retreated between 1996 and 2001, 11.8 can be attributed to the growth effect, and only 1.8 to the redistribution effect. It is the cities that have shown the sharpest decline. The incidence of urban poverty has retreated by 19.3 percentage points, compared to 9.8 in the countryside. Yet the advantage that cities have over the countryside in this regard is due to a significant shift in income distribution among urban dwellers. In fact, the redistribution effect has pushed down the incidence of urban poverty by 8.4 percentage points. At the same time, it is gratifying to note that economic growth has benefited urban and rural dwellers alike, accounting in both cases for 11.8 percentage points of the total decline.

\begin{tabular}{ccccccc}
\hline \multicolumn{6}{|c}{ Table 3 : Breakdown of the trend of poverty indicators between $\mathbf{1 9 9 6}$ and $\mathbf{2 0 0 1}(\mathbf{\%})$} \\
\cline { 2 - 7 } & \multicolumn{2}{c}{ Total change } & \multicolumn{2}{c}{ Growth effect } & \multicolumn{2}{c}{ Redistribution effect } \\
\cline { 2 - 7 } & P0 & P1 & P0 & P1 & P0 & P1 \\
\hline Rural & -9.8 & -3.1 & -13.3 & -6.6 & 1.7 & 3.4 \\
Urban & -19.3 & -8.3 & -9.0 & -4.7 & -8.4 & -4.7 \\
Total & -13.1 & -4.9 & -11.8 & -5.9 & -1.8 & 0.6 \\
\hline
\end{tabular}

Source: ECAM I, ECAM II

A similar analysis can be applied to the depth of poverty (P1), which has retreated nationally by 5.9 percentage points, thanks essentially to the growth effect. Again, the cities have shown a sharper decline $(8.3$ percentage points). This is attributable above all to economic growth and, secondarily, to the reduction in income disparities.

The severity of poverty (P2) has also declined nationally by 2 percentage points. This decline was weak in rural areas, and can be attributed to economic growth, while it was fairly strong in the cities, where it is attributable as much to a reduction in income inequality as it is to growth.

Source : MINEPAT/DSCN

\subsubsection{3 "Monetary" inequality in Cameroon}

123. An analysis of the poverty incidence trend is not enough, by itself, to appreciate fully the progress that has been made in reducing poverty. It needs to be supplemented by an analysis of the trend in income distribution disparities, in order to have a complete picture of the dynamics of monetary poverty. In fact, it may be that a relatively equitable income distribution, or a high concentration of households around the mean income and the poverty threshold, is what explains the apparent improvement in poverty incidence.

124. An analysis of income distribution in Cameroon using the ECAM II results shows that there are considerable gaps between the poor and the nonpoor, on one hand, and between urban and rural dwellers on the other. It also suggests that there was little narrowing of inequalities between 1996 and 2001. In fact, average annual income, estimated by mean expenditure per adult equivalent, is eight times higher among the nonpoor as among the poor 
(963,882 CFA francs versus 85,495 CFA francs in 2001), and it is twice as high in the cities as in the countryside (408,115 CFA francs versus 233,734 CFA francs).

125. Yet the gap between the poor and the nonpoor is more pronounced in the cities than in the countryside, indicating that urban income distribution is more highly skewed. Poor rural households devote more than 56 percent of their income to food. Overall, households spend 7.6 percent of their total annual budget on health, and 5.4 percent on education.

\section{Box 4. Measuring inequality}

In the economic literature, it is often recognized that growth can coexist with poverty, if there are no appropriate income distribution policies in place. Such a situation generally translates into a worsening, or at best a stagnation, of income inequalities. Similarly, a more equitable distribution of national income from one year to the next will tend to reduce poverty.

From the results of ECAM I and II, it can be seen that the decline in poverty has not been followed by any narrowing of inequalities. Indicators of inequality, such as the GINI index, log variance and the coefficient of dispersal between the first and last quintiles show that, despite poverty's retreat, inequalities, far from shrinking, have remained constant and have in some cases increased. For example, in 1996 the richest 20 percent consumed seven times as much as the poorest 20 percent; in 2001 , this ratio was eight to one.

These results reinforce the need to accompany growth policies with appropriate policies for distributing the fruits of that growth among all segments of the population. In particular, rural development policies and strategies must recognize these results and seek to correct disparities between cities and countryside.

\section{Table 4 : Income inequalities : GINI index and average expenditure by quintile}

\begin{tabular}{|c|c|c|c|c|c|c|}
\hline & \multicolumn{3}{|c|}{1996} & \multicolumn{3}{|c|}{2001} \\
\hline & Urban & Rural & Total & Urban & Rural & Total \\
\hline Gini index & 0.449 & 0.345 & 0.406 & 0.406 & 0.369 & 0.408 \\
\hline \multicolumn{7}{|c|}{ Average expenditure by quintile (CFA francs) } \\
\hline Poor (bottom $20 \%$ ) & 77,824 & 80,398 & 79,724 & 94,166 & 83,956 & 85,495 \\
\hline Rich (top 20\%) & 66,3805 & 491,856 & 585,168 & 758,960 & 600,618 & 693,882 \\
\hline Total & 322,421 & 200,805 & 243,240 & 408,115 & 233,734 & 294,403 \\
\hline
\end{tabular}

Source : DSCN : ECAM I and II Report

Source: MINEPAT/DSCN

126. A dynamic analysis indicates that, while the incidence of absolute poverty declined over the last five years, this cannot be said of inequalities. In fact, the ratio of the average annual income of the richest 20 percent of households (the top quintile) to that of the poorest 20 percent (bottom quintile) remained high and stable at a factor of about 8 , thereby confirming the robustness of income inequalities. The Gini index also remained stable and relatively high during this period (0.406 in 1996 and 0.408 in 2001). This places Cameroon in the upper midrange of countries in the region in terms of income inequality.

127. The significant progress reflected by the decline in poverty incidence shows clearly that it is sensitive to growth and to welfare policies. Yet the persistence of a high level of inequality also indicates that there is ground to be made up in terms of the impact of policies on poverty. The policies and strategies to be followed in the context of poverty reduction, then, must be designed both to intensify growth, in order to raise average incomes, and to ensure a better distribution of the fruits of growth. 


\subsubsection{The sociodemographics of poverty}

128. Analyzing poverty according to sociodemographic variables allows us to differentiate by sex and age of the household head, as well as by household size. Such an analysis also affords a better understanding of the phenomenon of poverty, and allows for better targeting of policies and actions to reduce this scourge. For example, a policy to promote subsistence crops as a means to food self-sufficiency and income generation could be specifically targeted at women, both as producers and as people who are particularly vulnerable.

\subsubsection{Poverty and the sex and age of the household head}

129. An analysis by sex of the household head yields encouraging

information about the gender gap. The incidence of poverty in households headed by women is slightly lower than that of households headed by men (38.7 percent versus 40.6 percent). It

Table 5 : Poverty incidence by sex and age of household head (\%)

\begin{tabular}{|lllllll|}
\hline & & 1996 & & 2001 & \\
\hline & Urban & Rural & Total & Urban & Rural & Total \\
\hline Sex & & & & & & \\
Male & 42.4 & 60.0 & 54.2 & 22.5 & 49.7 & 40.6 \\
Female & 36.4 & 55.8 & 45.8 & 20.7 & 50.6 & 38.7 \\
Total & 41.4 & 59.6 & 53.3 & 22.1 & 49.9 & 40.2 \\
\hline Age & & & & & & \\
$>30$ & 32.9 & 39.6 & 36.6 & 15.4 & 39.9 & 31.1 \\
years & & & & & & \\
$30-39$ & 35.6 & 48.2 & 42.3 & 17.2 & 45.6 & 34.4 \\
$40-49$ & 35.6 & 56.0 & 45.7 & 21.3 & 48.1 & 37.7 \\
50 year & 46.9 & 65.6 & 60.1 & 26.1 & 53.7 & 44.7 \\
+ & & & & & \\
Total & 41.4 & 59.6 & 53.3 & 22.1 & 49.9 & 40.2 \\
\hline Source: MINEPAT/DSCN : ECAM I and II Reports & & & \\
\hline
\end{tabular}

however, that 3 out of 4 households are headed by men, and that nearly 8 out of 10 poor people live in households headed by men.

130. An analysis by age of the household head shows that the poverty rate rises with the age of the household head, regardless of place of residence. This situation was observable both in 1996 and in 2001. Moreover, the poverty rate rises with household size: poor households have an average of 6.6 members, while the average for nonpoor households is 4.2.

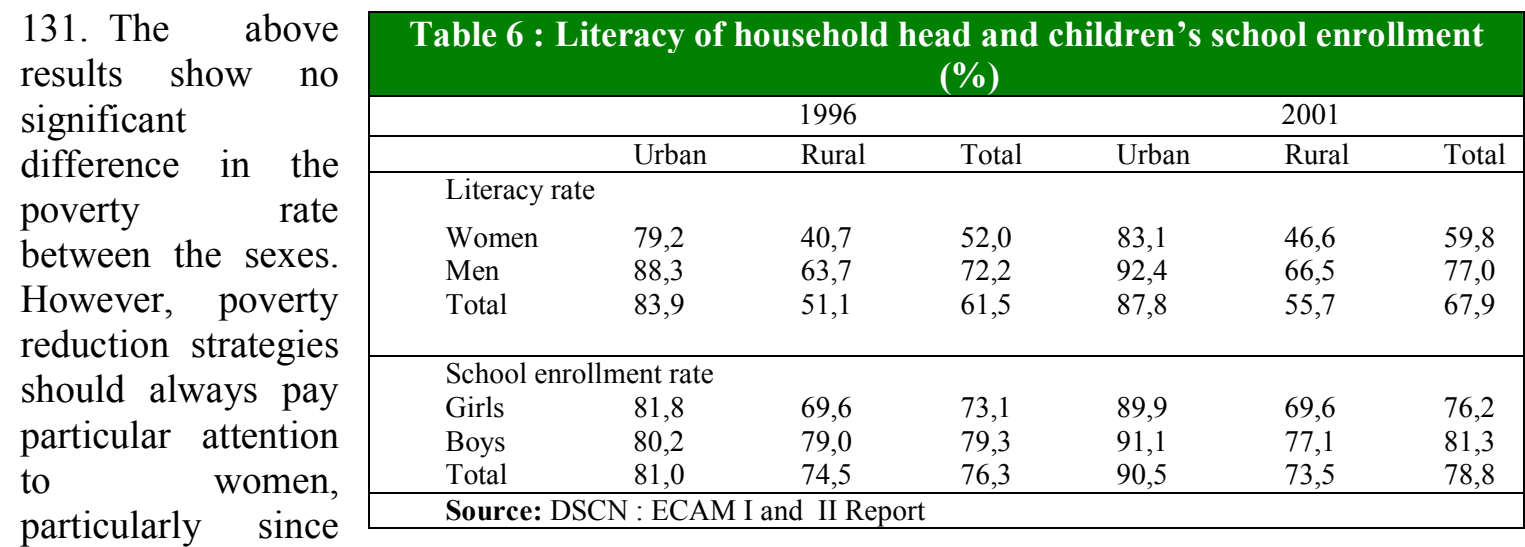

analysis using other indicators reveals great discrepancies between the sexes. For example, school enrollment indicators show women at a disadvantage. Specifically, the adult literacy rate is 59.8 percent for women vs. 77 percent for men, the school enrollment rate is 76.2 percent for girls ages 6 to 14 vs. 81.3 percent for boys in the same age bracket, and only 3.5 
percent of persons living in a female-headed household have access to formal credit, vs. 5.5 percent for those in male-headed households. These elements affect the relative ability of the sexes to generate incomes over the medium-term.

\subsubsection{Poverty and the household head's education level and economic activity}

132. The survey results also show that socioeconomic variables have a significant effect on the profile of poverty in Cameroon. Those with less education, small farmers, farm laborers, and non-management wage earners in the public sector are particularly affected by monetary poverty. Naturally, all of these variables are correlated with the fundamental factors of education and economic activity.

133. As a reflection of the importance of

education among poor households, 40 percent have no schooling (see contribution). Among the total unschooled population, one household in two is poor. This poverty incidence declines as the household head's level of education rises. It is clear, then, both for 1996 and 2001, that the higher the level of education, the greater the chances to earn income and to escape from poverty.

134. The occupation of the household head also appears to be an important factor distinguishing the poor from the nonpoor. Households where the head is a farmer are the category most affected by poverty, with an incidence of 57 percent. These households accounted for nearly 7 poor people out of 10 . Next to this group of households comes the group where the head works in the informal agriculture sector, with an incidence of 54 percent. The households best protected against poverty are those where the head is either a manager in the public or private sector, an employee in the formal private sector, or a proprietor in the informal nonfarm sector. The poverty incidence for these households varies between 7 and 22 percent. Yet non-management public sector employees suffer a poverty incidence of 25 percent or one person in four. This result shows the profound inequalities between the senior ranks and other employees in the public sector.
Table 8 : Poverty incidence by household head's occupational category

\begin{tabular}{|l|c|c|}
\hline & Incidence & Contribution \\
\hline $\begin{array}{l}\text { Senior/managerial } \\
\text { public sector }\end{array}$ & 6.9 & 0.9 \\
$\begin{array}{l}\text { Other public } \\
\text { employees }\end{array}$ & 24.9 & 3.7 \\
$\begin{array}{l}\text { Senior/managerial } \\
\text { private sector }\end{array}$ & 11.3 & 1.6 \\
Other private sector & 16.8 & 2.4 \\
employees & 57.0 & 66.6 \\
$\begin{array}{l}\text { Farmers } \\
\text { Informal farm }\end{array}$ & 54.2 & 2.0 \\
workers & 22.3 & 0.6 \\
Informal nonfarm & 34.4 & 10.4 \\
proprietors & 27.1 & 3.3 \\
Nonfarm self- & 25.0 & 2.9 \\
employed & 13.5 & 0.1 \\
Nonfarm employees & 18.4 & 1.0 \\
Unemployed & 50.6 & 3.2 \\
$\begin{array}{l}\text { Students } \\
\text { Retirees }\end{array}$ & 40.9 & 1.4 \\
$\begin{array}{l}\text { Disabled } \\
\text { Others not in }\end{array}$ & 40.2 & 100 \\
workforce & & \\
Total & & \\
Source: MINEPAT/DSCN : ECAM II Reports \\
\hline
\end{tabular}




\section{Box 5. Vulnerability}

An objective analysis of vulnerability consists of studying the consequences of an exogenous shock on the household living standard indicator. For example, how much would poverty incidence vary if the monetary poverty threshold were raised or lowered by the five percent?

Such an analysis is useful in studying variability. For example, take two different types of household, one headed by a public sector employee or a formal private sector worker, living below the poverty threshold but with a fairly stable income, and a farmer's family that is above the threshold but sees sharp fluctuations in its income (because of poor climatic conditions, difficulties in getting the produced a market, etc.). A standard static analysis of poverty would class the first household among the poor and the second among the nonpoor. Yet both are exposed to a certain degree of vulnerability, the first because it cannot attain the poverty threshold and the second because it must survive periods of extremely low income. If the farmer's family has no access to instruments for smoothing its consumption pattern or raising its income, it may need a safety net of some kind in order not to fall into poverty. On the other hand, the civil servant's family needs a different type of protection, in the form perhaps of higher wages in the public service.

Testing this hypothesis with ECAM II data shows that the poverty incidence would fall or rise by three percentage points. In fact, if for some reason the general price level were to fall by, say, five percent, some households would see their purchasing power increase. The distribution of this windfall among socioeconomic groups shows that 59,389 people in households headed by a public or formal private sector worker, and 70,287 in households where the head is not working or unemployed, would emerge from poverty, and poverty incidence would decline by 1.7 percent and 4.1 percent respectively. The latter category of households derive their income essentially from the extended family network: indeed, 8 to 10 disabled people are cared for by relatives. Retirees constitute a potentially vulnerable category because of their age. On the other hand, with inflation of five percent, for 266,636 people living in households where the head is a farmworker, the minimal needs satisfaction threshold will now be out of their reach, and they will fall below the poverty threshold, with an increase of 3.6 percent in the incidence of poverty.

Table 9 : Variation in poverty incidence following a 5\% change in the poverty threshold

\begin{tabular}{lllll}
\hline & \multicolumn{2}{l}{ Reduction of 5\% } & \multicolumn{2}{l}{ Increase of 5\% } \\
\cline { 2 - 5 } & $\begin{array}{l}\text { Number } \\
\text { affected }\end{array}$ & $\begin{array}{l}\text { Incidence } \\
(\%)\end{array}$ & $\begin{array}{l}\text { Number } \\
\text { affected }\end{array}$ & $\begin{array}{l}\text { Incidence } \\
(\%)\end{array}$ \\
\hline Public/formal private sector workers & 59,389 & 1.7 & 83,439 & 2.4 \\
Farmers & 262,148 & 3.5 & 266,636 & 3.6 \\
Informal workers & 87,007 & 3.1 & 88,225 & 3.1 \\
Not working/unemployed & 70,287 & 4.1 & 46,190 & 2.7 \\
Total & 478,831 & 3.1 & 484,490 & 3.1 \\
\hline
\end{tabular}

Source: DSCN/ECAM II

\subsubsection{The determinants of poverty}

135. Analyses using ECAM II data have identified the principal determinants of poverty, which include education level of the household head, socioeconomic group, and place of residence. Thus, it appears that households where the head has little education are the most vulnerable. The incidence of poverty among households where the head has some higher education is 4.1 percent, compared with 42.5 percent for those where the head has no education. 


\section{Box 6. Identifying the principal determinants of poverty in Cameroon}

- The problem. Formulating and implementing an effective poverty strategy requires an accurate identification of the main factors that "cause" or perpetuate poverty. The preceding analyses, using simple tabulation methods, can help in this identification. Yet the "one factor at a time" method has limitations, because of correlations and interactions between factors. For example, "economic occupation" may reflect the "education" effect, and it may well be that the interaction between "education" and "occupation" is the determining element, since individuals with the same level of education may have different levels of income, depending on the sector in which they work (industry or services, public or private, etc.).

- Methodology. To estimate the contribution of these different determinants, while taking account of marginal effects and interactions between factors, econometric models are often used to explain poverty. Such a model was estimated for Cameroon using data from ECAM II. After several iterations that took account of correlations and interactions between factors, the following variables were selected from the model as "explaining" monetary poverty: (i) place of residence (urban/rural); (ii) household head's level of education; (iii) household head's socioeconomic category; (iv) household head's sex; (v) form of water supply; (vi) type of toilet; (vii) size of household; and (viii) the time needed to reach the nearest paved or all-season road from home.

- Results. The results of the model (see annex) confirm the descriptive analyses of the ECAM II results, and are also consistent with the results of the participatory consultations. They provide a better understanding of how the various phases of poverty interact, and of their relative importance.

Level of education turns it to be a very significant explanatory factor. In fact, ceteris paribus, a household where the head has some higher education is six times more likely to escape from poverty than a household where the head has no education. A poverty strategy, then, should place particular stress on human capital formation.

The household head's sector of occupation is just as important. Households where the head works in the public or the formal private sectors are better off than others. This finding is to be expected, since these workers enjoy an adequate degree of social protection. Other socioeconomic groups (farmers, informal workers, those not working or unemployed) are at higher risk. Farmers are the most affected by poverty: they are three times more likely to be poor than are families where the head works in the public or formal private sector.

The physical environment also has a significant impact on household living standards. Apart from geographic conditions, which are assumed to be exogenous to the model, the lack of economic infrastructure, and in particular the poor state of urban [sic] roads and the isolation of villages, makes life difficult for families. This is reflected in the negative coefficient of the time that it takes a rural dweller to reach the nearest paved or all season road.

- Strategy implications. The results of this analysis confirm those of the quantitative and qualitative surveys on the key components of a strategy for reducing poverty, in particular: (i) education and health for strengthening human capital; (ii) growth, particularly in the formal modern sector, for generating employment and income; and (iii) accessible infrastructure (roads, water, etc.).

Source : MINEPAT/DSCN

136. The socioeconomic category of the household head also has a considerable influence on family living standards. Household heads working in the formal private or public sectors have less chance of being poor, to the extent that they enjoy an adequate degree of social protection. Other groups (farmers, informal workers, those not working or unemployed) are more exposed. Farmers run the greatest risk of poverty: they are three times more likely to be poor than are families where the head works in the public or formal private sector.

137. Finally, rural residence seems to be a risk factor. The poorest families live in the countryside. Yet it is important to note that here the environment has some influence on 
family living standards: apart from geographic conditions, which are assumed to be exogenous, the lack of infrastructure, and in particular the isolation of towns and villages, makes life difficult for the families living in them. The distance to the nearest paved road has in fact been identified as a source of family impoverishment.

\subsection{PARTICIPATORY ASSESSMENT OF POVERTY IN CAMEROON}

138. With a view to developing a credible poverty reduction strategy that would incorporate contributions from all development stakeholders, the government adopted a participatory approach that involved representatives of (i) public and parapublic administrations, including parliamentarians, local government and administrative workers, (ii) economic operators in the private sector, (iii) labor unions and professional organizations, and (iv) civil society organizations, specifically NGOs, community-based initiative groups (groupements d'initative commune, GIC), savings and loan cooperatives (COOPEC), community credit unions (mutuelles communautaires de crédit, MC2), women's associations and youth groups, various organizations (street children, the handicapped, etc.), specific groups (Bororo, Pygmies, fishing communities, etc.), development committees and religious congregations.

139. This approach, which is complementary to the quantitative or monetary approach, is based on participatory consultations designed to understand the manifestations and characteristics of poverty as they are perceived by poor people themselves. The authorities felt that such an understanding was essential for targeting pockets of poverty and for deciding the most appropriate actions to take in light of the needs expressed by the populations concerned. Thanks to this understanding, the government will also have a better appreciation of the nature and volume of resources that must be mobilized for priority projects and programs to combat poverty.

\subsubsection{The main stages of the participatory process for preparing the PRSP}

140. The participatory process for preparing the national poverty reduction strategy ${ }^{7}$ was launched in January 2000 at ECAM, during a seminar-workshop that brought together representatives of public and parapublic administrations, universities, NGOs, religious organizations and donors. This seminar allowed for an initial exchange of views on the determinants of poverty in Cameroon, and on the main components of a strategy for reducing that scourge. At the same time, it sparked deeper thinking about the methodology for organizing participatory consultations at the grassroots level.

141. From March 31 to April 10, 2000, an initial round of participatory consultations took place in all 58 departments of the country, involving 203 target groups representing the various components of civil society, as well as individual activists. In total, nearly 10,000 people, 40 percent of whom were women, expressed their views during these consultations. Discussions were completely open, and were conducted in the presence of observers from the IMF, the World Bank, and the German cooperation agency GTZ. These consultations produced a mass of highly interesting and useful information about the way poverty is viewed at the grassroots level, the way people analyze the causes of poverty, and their proposed solutions for reducing it. The results of this first phase of participatory consultations were presented during a national workshop in May 2000, which brought together representatives of civil society, and in particular those of the groups consulted. This workshop identified the key

\footnotetext{
${ }^{7}$ See Annex 2.1.
} 
themes that should be developed in the Interim Poverty Reduction Strategy Paper (I-PRSP). That paper was presented in October 2000 during a seminar that reinforced the contribution of the private sector and civil society to preparing the PRSP, and laid the basis for those key players to participate in subsequent monitoring and evaluation of the strategy during its implementation.

142. In January 2002, the second round of participatory consultations took place in all 58 departments of Cameroon, involving about 6000 individuals from different social categories. Those consultations allowed people, in particular the poor, (i) to supplement, refine and prioritize the major elements of the regional poverty profile as they emerged from the participatory consultations of March and April 2000, (ii) to learn about poverty reduction activities and strategies planned under the HIPC initiative, (iii) to make suggestions for improving the interim PRSP, and to put forward ideas for poverty reduction projects, and (iv) to discuss the initial ideas on the configuration and operation of the National Poverty Reduction Network. In April 2002, the results of the second round of participatory consultations were presented at a national feedback seminar.

143. From June 26 to July 7, 2002, a working retreat was organized at Limbé for drafting the full PRSP. This work was performed by a multidisciplinary team consisting of national experts and advisers from the public administrations concerned, as well as experts from civil society. The elements emerging from that work were compiled by the Technical Monitoring Committee for Economic Programs (CTS) and finalized during a second retreat at Kribi, from November 28 to December 3, 2002. Between December 6 and 10, 2002, the government transmitted the draft PRSP resulting from the Kribi retreat to civil society representatives, private sector economic operators, donors and governments, requesting their observations or suggestions for improving the paper. Responses were numerous, and most of the proposals received were taken into account, in order to improve the document and ensure a high-quality PRSP.

144. From December 16 to 19,2002 , a public workshop was held to formulate input by civil society to the draft PRSP. At that workshop, participants had the opportunity to: (i) understand the process of preparing and implementing the PRSP, (ii) examine the role and place of civil society in implementing the interim PRSP, (iii) determine a strategy for presenting the contributions of that workshop to other members of civil society, to the government and to donors, and (iv) prepare a strategy for involving civil society as a partner in implementing the PRSP. On December 27, 2002, a seminar was held to allow civil society organizations and private sector operators to exchange views. At that seminar, the main players in poverty reduction provided comments on the draft PRSP, with a view to making last-minute improvements to it before its submission to the Executive Boards of the World Bank and the International Monetary Fund. On March 14, 2003, the paper was presented to the donor community.

\subsubsection{How people perceive poverty}

145. The participatory consultations provided a better appreciation of how people perceive poverty in their daily lives (see annex 2.2). As with the quantitative assessment, incomerelated elements are seen as the most important characteristics of poverty in Cameroon. As people put it, "being poor means not having the money to satisfy basic needs", in particular for food, housing, health care and education. 
146. Other dimensions of poverty emerging from the quantitative approach (education, health, roads, drinking water etc.) are also clearly perceived by the public, according to the results from the participatory consultations. People recognize that, even with adequate incomes, the lack of essential services can keep them poor: "Poverty means trouble in getting safe drinking water, electricity, staple products, it means lack of roads and means of communication"; "poverty is when schools are far away, and when the schools offer no real teaching"; "a poor person is one who is in bad health and has trouble finding medical care".

147. Social dysfunctioning is also seen as a characteristic of poverty. This term covers a number of failures, not necessarily related to financial capacity. As people put it, poverty is characterized by the collapse of morals (delinquency, prostitution, etc.), the loss of selfesteem and of social standing. Poverty also means living with insecurity, crime and unsanitary conditions. It means the inability to seek justice, to enforce rights, and to avoid everyday abuses.

\subsubsection{How people see the determinants of poverty}

148. The participatory consultations helped to identify the "factors" of poverty as seen by poor people themselves. The isolation that some regions suffer through the lack of highways and rural roads was mentioned by the great majority of people consulted as one of the major causes of poverty. This isolation makes it impossible for people in these regions to get their produce to market or to obtain provisions at reasonable cost. More generally, this concern goes hand-in-hand with the problem of access to basic infrastructure.

149. People also complained about the deregulation of many farm products and the lack of adequate policies to support farmers, as further factors for impoverishment. With no support or extension services, farmers have suffered negative fallout from restructuring, in the form of higher prices for inputs and low prices for their produce, in the absence of credit or advances to producers, and great price volatility. People have a feeling that deregulation was not sufficiently thought out, and that it should not imply the total elimination of state support for farmers and rural economy.

150. Bad governance is also cited as a factor for impoverishment. People pointed in particular to corruption, the diversion of public funds, impunity, exorbitant charges for public services, and the failure to decentralize, as factors contributing to the unfair distribution of the fruits of growth and to the persistence of poverty in Cameroon.

151. The fact that some regions of the country are poorly served by communication infrastructure is also seen as a cause of poverty. People there have a feeling that they are cut off from the rest of the country and from the flow of information from the outside world. The inadequacy of social infrastructure and facilities in education and health, and understaffing in those sectors, particularly in rural areas, are seen as a constraint on people's self-fulfillment.

152. Environmental problems specific to certain regions, such as drought, water problems and migratory birds, were also cited as factors of poverty. As well, problems of land title and access, conflicts between farmers and herders, the lack of administrative and economic support, particularly in the forest, coastal and frontier areas, were also commonly evoked as factors of poverty. 
153. Social dysfunctioning, which is seen first of all as a characteristic of poverty, is also cited as a factor of poverty. This term covers a number of failures, not necessarily of a material nature. It involves the collapse of moral traditions, the loss of self-esteem, the decline of family or community solidarity, the lack of public spirit and the resurgence of tribalism and sectarianism, the prevalence of prejudices against social groups with different cultures and ways of life. Insecurity in the face of full-scale banditry and petty crime, HIV/AIDS, early marriage and premature motherhood, and polygamy are also cited as factors contributing to poverty.

\subsubsection{How people would tackle poverty}

154. People proposed a number of solutions for reducing poverty. ${ }^{8}$ Those solutions rely essentially on strategies for generating incomes and improving living standards. Thus, people want the government to continue providing rural extension services and financing for modernizing techniques in farming, fishing and cottage industries, to promote research into the diversification and processing of farming and livestock products, to create processing and conservation facilities so that farm products can be safely taken to market in the major centers in short, to take all the steps necessary to improve living standards in the countryside and stem the rural exodus. Next, they propose adopting an industrial development strategy for mining and forest resources which will promote the creation of processing units that could become real poles of development and attraction for new businesses in many parts of the country. Finally, the proposed solutions extend to rural water and electrification programs, intensifying electrical grids by building micro-dams, and development of sources of drinking water throughout the country.

155. In the education and health sectors, the principal recommendations deal with the equipment, construction and rehabilitation of school and health facilities. People want to see more and better personnel resources devoted to education and health, the construction of new universities attuned to local realities, the reform of school and university curricula to match them with employment opportunities, stressing civics and moral issues as much as technical aspects. Finally, they are asking for greater national solidarity in combating contagious diseases, better care for HIV/AIDS victims, a stepped-up campaign against HIV/AIDS, malaria, meningitis and viral hepatitis, and supplying health centers with generic drugs that are affordable to all segments of the population.

156. Transportation and communication infrastructure features prominently in people's demands. They want isolation to be broken down in every region, through the rehabilitation and maintenance of the existing road network, the paving of major national and interprovincial highways, the expansion and upkeep of rural roads so that farmers can get their output to market, nationwide coverage for communication systems, particularly in the frontier area (radio, television, telephone and other means), and the promotion of information and communication technologies (ICTs).

157. Many recommendations dealt with governance. People proposed restoring government authority, proceeding further with decentralization in the management of public affairs, stepping up the campaign against corruption, reinstituting civics education in school curricula, and strengthening the ability of the police forces to combat insecurity and enforce the law for all.

\footnotetext{
${ }^{8}$ See Annex 2.3
} 


\subsubsection{Monitoring and assessment mechanisms favored during the consultations}

158. The January 2002 consultations provided the opportunity not only to present people with a list of indicators for monitoring poverty reduction policies and activities, but also to share with them the ideas for instituting a National Poverty Reduction Network for the exchange of information on poverty. People welcomed this initiative.

159. They also proposed that (i) the network should have a pyramidal structure, with the village, commune or city district (arrondissement) at the base, (ii) information should be circulated using existing local transmission methods, reinforced by modern communication techniques, and (iii) awareness, information, education and communication strategies should be targeted at the poor in order to explain to them the rationale for the National Poverty Reduction Network and how it will work.

\subsection{THE OTHER DIMENSIONS OF POVERTY AND HUMAN DEVELOPMENT IN CAMEROON}

160. Education, health, and infrastructure services (such as energy, roads, drinking water) constitute a set of essential social goods and services that, together with income, contribute to improving the quality of life. Access to these essential services is a direct indicator of people's well-being. Essential social services contribute to the poverty reduction strategy in two ways: they determine the quality of life, on one hand, and they also help to improve people's ability to earn income and lift themselves out of poverty.

\section{Box 7. Some school enrollment indicators defined}

- The gross enrollment rate compares the number of students registered in primary school to the total population of primary school age. It measures the capacity needed to enroll all children. It tends to overestimate effective coverage, however, because of late enrollment and repetition. This indicator is a mean value that says nothing about how many pupils complete primary school.

- The rate of access to CM2 (the final year of primary school), or the completion rate, measures the percentage of children who complete primary school, and would seem to be more appropriate for measuring progress towards universal primary education.

- The gross primary school access rate measures the ratio of new entrants (non-repeaters) in the first year of primary school to the total population of children of first-year primary school age.

- The retention rate in CM2 measures the percentage of pupils entering the first year of primary school who reach the sixth grade.

- The enrollment profile measures the average school career of individuals under current enrollment conditions. For a cohort of 100 children, it shows the proportion newly registered in each year of study.

Source: MINEDUC

\subsubsection{Education, training and poverty reduction}

161. Overall trends portray a mixed picture for the situation and progress of the education sector. There has been a slight improvement, represented by an increase of about 2.5 percentage points in the overall net enrollment rate between 1995/1996 and 2000/2001 (76.3 percent to 78.8 percent). This trend is consistent with that of the literacy rate, which has gained 6.4 percentage points over the same period (61.5 to 67.9 percent). More encouraging is the fact that the enrollment rate for girls has risen faster than that for boys, recording a significant catch-up of 1.1 percentage point and confirming that Cameroon has been successful in reducing gender disparities and offering economic opportunity. 
162. The enrollment profile measures the effective coverage of each level of schooling, on the basis of school attendance and the completion rate. In 2001, the rate of access to primary school stood at 95 percent, which is close to the target of 100 percent access to the first year of primary school (SIL/CII), and represents an increase of 5 percentage points

\begin{tabular}{|lll|}
\hline \multicolumn{3}{|c|}{$\begin{array}{c}\text { Table } 10 \\
\text { : Key indicators in primary } \\
\text { education (\%) }\end{array}$} \\
\hline Access rate & 2000 & 2001 \\
Completion rate & 90 & 95 \\
Repetition rate & 43 & 56 \\
Source: MINEDUC & 27 & 25 \\
\hline
\end{tabular}
over the year 2000. On the other hand, the completion rate for primary school is still low (56 percent), which means that of every 100 new entrants into primary school, only 56 reach CM2 (the sustainable literacy threshold). In fact, the dropout rate in the first two years (SIL and CP) of primary school is very high. There is a sharp decline in the access rate into secondary school: the transition rate from CM2 to grade 6 is about 60 percent, which means, roughly, that only two out of three new entrants into CM2 will go on to the sixth year of general education. The completion rate for the first cycle of secondary school in 2000/2001 was 67 percent.

163. The quality of the education system. Educational quality is measured not only by academic results but also by learning conditions and the student-teacher ratio. The quality indicators show a deterioration in both these areas. The success rate for the CEPE/FLSC, the examination marking the end of primary school, was 72 percent in 2001 . The repetition rate remains high 25 percent, 21 percent and 31 percent in primary school, the first cycle of secondary, and the second cycle of secondary, respectively. The ratio of pupils to classrooms is 52, ranging from 38 in the South Province to 74 in the Extreme North.

- Teachers. The student-teacher ratio in primary education was 63 during the school year 2000/2001. It ranged from 37 in the South Province to 77 in the Extreme North. Nearly one-half of the secondary school teaching body is underqualified, because of shortcomings either in initial training or in continuous professional development. Moreover, the education supervision system is weak (lack of training for supervisors, too few supervisors, little mobility for supervisors because of logistic constraints, etc.) and does little to improve the situation. To all this must be added a general lack of morale among teachers, because of discrepancies in their status (some enjoy tenure, while others are on temporary contract and are paid much less) and poor working conditions, particularly in the larger cities (where class sizes sometimes reach 100 pupils).

- Shortages of textbooks and teaching materials. Teachers and students alike suffer from a shortage of essential textbooks: with the exception of French and mathematics (for which seven students out of 10 , and five students out of 10, respectively, have the required textbooks), the textbook possession rate for other subjects is only three students in 10. As for the teachers, the shortage of teaching texts is even more glaring: only one teacher in two has a French teaching guide, 3 in 10 have the mathematics guide, and one in 10 has the science guide.

\footnotetext{
${ }^{9}$ The data sources for 2000 and 2001 are different. For 2000, data are taken from the 99/00 survey of Regional Departments of Education, while for 2001 there was an exhaustive survey of all educational institutions.
} 
- The lack of basic facilities (drinking water, electricity, toilets) and specialized classrooms in most schools is a further handicap.

- Health conditions are also of concern (there is no effective school health policy: no infirmary, no basic medications, etc.), and are made worse by the HIV/AIDS epidemic in the schools.

\section{Poverty and level of education}

The foregoing analysis suggests that education conditions for the population as a whole are a cause of concern. Analyses of the poverty profile show that schooling conditions for the poor are even more alarming. These results confirm that there is a significant

\begin{tabular}{|c|c|c|c|c|c|c|}
\hline & \multicolumn{3}{|c|}{1996} & \multicolumn{3}{|c|}{2001} \\
\hline & Urban & Rural & Total & Urban & Rural & Total \\
\hline \multicolumn{7}{|c|}{ Net enrollment rate $(\%)$} \\
\hline Poor & 74,1 & 78,1 & 77,2 & 82,6 & 72,3 & 74,1 \\
\hline Nonpoor & 87,0 & 67,7 & 74,9 & 93,2 & 75,0 & 82,9 \\
\hline Total & 81,0 & 74,5 & 76,3 & 90,5 & 73,5 & 78,8 \\
\hline \multicolumn{7}{|c|}{ Average education expenditure (CFAF) } \\
\hline Poor & 23269 & 14876 & 17314 & 27545 & 13525 & 15973 \\
\hline Nonpoor & 61650 & 28110 & 47655 & 86614 & 38513 & 68001 \\
\hline Total & 46787 & 19069 & 30692 & 79780 & 24810 & 48046 \\
\hline \multicolumn{7}{|c|}{ Share of education spending (\%) } \\
\hline Poor & 5,8 & 4,2 & 4,8 & 6,6 & 3,6 & 4,2 \\
\hline Nonpoor & 4,8 & 2,4 & 3,9 & 6,7 & 3,7 & 5,6 \\
\hline Total & 5,0 & 3,1 & 4,1 & 6,7 & 3,7 & 5,4 \\
\hline
\end{tabular}
link between schooling and poverty in Cameroon. In fact, the net enrollment rate is 8.8 percentage points lower for children ages 6 to 14 living in poor families, compared to those in nonpoor families (74.1 percent versus 82.9 percent in 2001). This discrepancy may reflect the fact that poor people do not have sufficient income to cover school expenses, and/or that access is more difficult for poor children than for the nonpoor, or in "regions" that are poor in relation to others. Moreover, education spending is five times higher among nonpoor families than among poor families $(68,001 \mathrm{CFA}$ francs versus 15,973 CFA francs, on average, in 2001): it accounts for 4.2 percent of poor family budgets, and 5.6 percent for nonpoor families. The difference in the enrollment rate is thus in large part a reflection of monetary poverty, but it also betrays inter-regional differences in capacities and hence in access.

\subsubsection{Health and poverty reduction}

165. General conditions. As with education, health is an important element not only for the quality of life but also for the productivity of the country's human resources. The principal health indicators deteriorated between 1991 and 1990. During that time, the infant mortality rate rose sharply by about 12 points, the chronic malnutrition rate for infants ages 12 to 23 months rose from 23 percent to 29 percent, and the rate of professionally assisted childbirth declined by 5 percentage points. Nevertheless, the vaccination rate (DTP3) improved by nearly 20 percentage points between 2001 and 2002, rising from 43 to 63 percent. 


\begin{tabular}{|lccc|c|}
\hline \multicolumn{5}{|c|}{ Table 12 : Selected public health indicators } \\
\hline Health indicators & $\mathbf{1 9 9 1}$ & $\mathbf{1 9 9 8}$ & Change & $\mathbf{2 0 0 1}$ \\
\hline & & & & \\
Infant mortality rate (per 1000) & 65 & 77 & -12 & \\
Chronic malnutrition rate (\%) & 23 & 29 & -6 & \\
Rate of low birth weight (\%) & 16 & 22 & -6 & \\
Maternal mortality rate (per 100 000 live births) & 430 & 430 & 0 & \\
Assisted childbirth rate (\%) & 63.8 & 58.2 & & \\
Vaccination rate (DTP3 in \%) & 34 & 48 & +14 & 43 \\
Utilization rate of public health facilities (\%) & 30 & & & \\
HIV/AIDS prevalence rate (\%) & 2 & 7.2 & -5.2 & 11.8 \\
\hline Source: MINEPAT/DSCN; EDS 1991 and 1998; Health Sector Strategy & & & \\
\hline
\end{tabular}

166. Indicators of the availability of health services show that much remains to be done in terms of training and recruiting medical and paramedical personnel, and in constructing and equipping health centers. For example, the per capita ratios of physicians, nurses, hospital beds, health centers and pharmacies shown in the

Table 13 : Service availability indicators

$\begin{array}{ll}\text { Doctors/10.000 } & 1 \\ \text { Nurses/3.000 } & 1.3 \\ \text { Beds/1000 } & 1.3 \\ \text { Health Centres/10.000 } & 1.2 \\ \text { Pharmacies } / 100.000 & 1.6\end{array}$

Source: EDS 1991 and 1992; Health Sector Strategy

above table indicate important shortfalls in comparison with WHO recommendations. In 2001, the human resource deficit was estimated at some 9000 persons. Moreover, health personnel are unevenly distributed throughout the country, there is little in the way of human resource planning, and working conditions mean that productivity is low. When it comes to infrastructure and equipment, much of the health system is outdated, facilities are unevenly distributed among the provinces, as well as between urban and rural areas, technical support centers are obsolete, and there is no proper management accounting system for health infrastructure and facilities. Finally, real maintenance expenditure is less than 2.5 percent of what is needed.

167. Public health spending in recent years has fallen far short of needs. For the year 2002, the health sector's needs were estimated at CFAF 320 billion, or 5 percent of GDP, while the health budget represented only 1.1 percent of GDP. Consequently, households continue to bear most of the burden of health costs: direct payment for services accounts for 76 percent of total financing, compared to 18.5 percent from the government, and 5.5 percent from external financing.

168. The epidemiological profile of Cameroon, like that in most countries of sub-Saharan Africa, is dominated by infectious and parasitic diseases. There is also a rising trend in the prevalence of certain pathologies such as hypertension, diabetes and cancer. Infectious diseases such as AIDS, malaria and tuberculosis account for a particularly high share of general morbidity. Health statistics show that malaria alone is responsible for 40 to 50 percent of medical consultations, 40 percent of deaths among children under five years, and 23 percent of hospitalizations, and it consumes about 40 percent of annual family health budgets. As well, there has been a resurgence of diseases that were once on the verge of disappearance, such as tuberculosis $(22,000$ new cases on average each year), often found in combination with the rising incidence of HIV/AIDS (30 percent of tuberculosis victims are also seropositive). Finally, the HIV/AIDS prevalence rate has risen alarmingly in Cameroon, from 0.5 percent in 1987 to 2 percent in 1992, and 11.8 percent in 2002, placing Cameroon number 11 among the countries most affected by this pandemic. 
169. Health and poverty in Cameroon. Apart from these overall trends, an analysis of the poverty profile shows significant differences between the poor and nonpoor in terms of their state of health and their access to care. Most people still turn to formal health services in case of illness. Among those who declared themselves ill in 2001, 3/4 were able to seek consultation at a formal health center, versus $1 / 4$ in informal facilities. Formal health centers are more frequently visited by the nonpoor, and informal facilities by the poor. ${ }^{10}$

\begin{tabular}{|c|c|c|c|c|c|c|}
\hline & \multicolumn{3}{|c|}{1996} & \multicolumn{3}{|c|}{2001} \\
\hline & Urban & Rural & Total & Urban & Rural & Total \\
\hline \multicolumn{7}{|c|}{ Consultation rate in formal facilities (\%) } \\
\hline Poor & 72.2 & 65.0 & 67.8 & 74.0 & 69.0 & 70.0 \\
\hline Nonpoor & 87.5 & 86.2 & 87.0 & 83.8 & 75.3 & 79.1 \\
\hline Total & 82.6 & 75.6 & 79.2 & 81.7 & 72.2 & 75.5 \\
\hline \multicolumn{7}{|c|}{ Consultation rate in informal facilities (\%) } \\
\hline Poor & 28.0 & 35.0 & 32.2 & 25.9 & 31 & 30.0 \\
\hline Nonpoor & 12.5 & 13.8 & 13.0 & 16.2 & 24.7 & 20.9 \\
\hline Total & 17.4 & 24.4 & 20.8 & 18.3 & 27.8 & 24.5 \\
\hline \multicolumn{7}{|c|}{ Immunization rate for infants 12 to 23 months (\%) } \\
\hline Poor & 68.1 & 66.9 & 67.2 & 53.1 & 46.5 & 47.1 \\
\hline Nonpoor & 89.5 & 27.2 & 57.9 & 70.2 & 54.5 & 61.2 \\
\hline Total & 80.3 & 53.4 & 63.3 & 67.0 & 50.3 & 55.3 \\
\hline \multicolumn{7}{|c|}{ Average health expenditure in CFA francs } \\
\hline Poor & 7342 & 5562 & 6044 & 8934 & 6671 & 6937 \\
\hline Nonpoor & 26035 & 15130 & 19903 & 45687 & 19825 & 32178 \\
\hline Total & 18301 & 9423 & 12521 & 39116 & 12922 & 22036 \\
\hline \multicolumn{7}{|c|}{ Household budget share of health expenditure (\%) } \\
\hline Poor & 6.8 & 7.5 & 7.3 & 6.5 & 8.6 & 8.5 \\
\hline Nonpoor & 6.6 & 8.2 & 7.2 & 6.2 & 6.7 & 6.5 \\
\hline Total & 6.6 & 8.0 & 7.2 & 6.2 & 7.9 & 7.6 \\
\hline \multicolumn{7}{|c|}{ Average distance to nearest health center $(\mathbf{k m})$} \\
\hline Nonpoor & & & & 1.10 & 4.96 & 3.31 \\
\hline Total & & & & 1.13 & 5.26 & 3.86 \\
\hline \multicolumn{7}{|c|}{ Source : DSCN : ECAM I and II Reports } \\
\hline
\end{tabular}

170. The vaccination rate for nonpoor children is better than that for poor children, and children are better protected in the cities than in the countryside. In 2001, slightly more than half (55.3 percent) of infants between 12 and 23 months received the complete EPI vaccination dose (source: ECAM). According to the Ministry of Health, that rate is 43 percent.

\footnotetext{
${ }^{10}$ These figures must be viewed in perspective, however, since many of the health facilities visited by patients operate outside official regulations, and are regarded by the Ministry of Public Health as being in the informal sector. As well, the Ministry, in contrast to the ECAM survey, considers private consultations in public health centers to be informal. This approach adds to the justification of the governance measures planned in the health sector strategy.
} 
171. The cost of health services rose nearly three times as fast as the average inflation rate over the last five years, by some 70 percent (from 13,000 CFA francs to 22,000 CFA francs), and it is apparent that financial capacity has a considerable impact on the demand for health services. Annual average health spending per capita is three times higher in urban than in rural areas (39,000 CFA francs vs. 13,000 CFA francs), and four times as high among the nonpoor as among the poor $(32,000$ CFA francs vs. 6,900 CFA francs).

172. According to regional health map data, 54 percent of people live less than five kilometers from an integrated health center. This average figure, however, conceals wide regional disparities, ranging from 43 percent in the province of Adamaoua to 78 percent in the province of Littoral. Moreover, the ECAM II report notes that rural people must travel five times as far as urban dwellers to reach the nearest health facility. Even more strikingly, 98.9 percent of people who must travel more than six kilometers to a health facility live in the countryside, indicating the serious problem of rural health care access.

\subsubsection{Basic infrastructure services}

173. As with health and education, safe drinking water and transportation and energy services, etc., are among the amenities of life. Infrastructure services also affect households' capacity to reach markets and to participate in economic activity.

174. Access to safe drinking water and energy. For the population as a whole, potable water consumption rose substantially over the period. One household in two had access to safe drinking water in 2001, compared to only one in five in 1996. Similarly,

\begin{tabular}{|c|c|c|c|c|}
\hline & \multicolumn{2}{|c|}{1996} & \multicolumn{2}{|c|}{2001} \\
\hline & Urban. & Rural. & Urban. & Rural. \\
\hline \multicolumn{5}{|c|}{ Drinking water (\%) } \\
\hline Poor & 56.6 & 22.4 & 71.5 & 28.2 \\
\hline Nonpoor & 81.0 & 28.4 & 88.3 & 33.4 \\
\hline Total & 73.6 & 25.5 & 86.2 & 31.3 \\
\hline \multicolumn{5}{|c|}{ Electricity (\%) } \\
\hline Poor & 61.4 & 9.6 & 68.2 & 14.9 \\
\hline Nonpoor & 82.9 & 13.6 & 91.0 & 29.0 \\
\hline Total & 76.3 & 11.7 & 88.2 & 23.4 \\
\hline \multicolumn{5}{|c|}{ Time to nearest paved road (minutes) } \\
\hline Poor & & & 8.4 & 75.1 \\
\hline Nonpoor & & & 6.0 & 62.0 \\
\hline Total & & & 6.3 & 67.1 \\
\hline \multicolumn{5}{|c|}{ Fixed telephone (\%) } \\
\hline Poor & & & 6.2 & 0.4 \\
\hline Nonpoor & & & 51.6 & 2.5 \\
\hline Total & & & 46 & 1.6 \\
\hline \multicolumn{5}{|c|}{ Source: MINEPAT/DSCN : ECAM I and II Reports } \\
\hline
\end{tabular}
nearly half of Cameroonian households (46 percent) make use of electric lighting.

175. Yet there are considerable discrepancies, both between urban and rural areas, and between poor and nonpoor households. For example, urban accessibility rates are three times as high as rural rates, both for water and for electricity. On the other hand, these gaps are insignificant as between poor people and the nonpoor, reflecting the "public" nature of these services: when they are available at all in a region or locality, they are accessible to the poor as well as to the nonpoor, consistent with the government's "social" approach to policy in these sectors. ${ }^{11}$

176. Road access. An essential element of the quality of life, and of people's ability to insert themselves in the economy, is the availability of properly maintained roads for transporting persons and goods. Isolation raises transaction costs and is a factor for poverty. Cameroon has

\footnotetext{
${ }^{11}$ The prices charged for water and electricity consumption include a "social" rate that is lower than the normal price.
} 
a relatively dense network of interurban roads spanning some 50,000 kilometers, 53 percent of which are classed as the primary or trunk network. Of this network, 16 percent is paved, nearly 40 percent consists of dirt roads, and 45 percent is non-engineered rural tracks. Traffic counts for the year 2001 show that paved roads and dirt roads together carried about 90 percent of traffic (62 percent and 27 percent). Data also show that nearly 80 percent of paved roads, 73 percent of dirt roads, and 70 percent of rural tracks are in the bad or mediocre condition.

\begin{tabular}{|c|c|c|c|c|c|c|c|c|c|}
\hline \multirow{2}{*}{\multicolumn{2}{|c|}{ Characteristics }} & \multicolumn{4}{|c|}{2000} & \multicolumn{4}{|c|}{2001} \\
\hline & & Paved & Dirt & Rural & Total & Paved & Dirt & Rural & Total \\
\hline \multirow[b]{2}{*}{ Length } & in $\mathbf{k m}$ & 3993 & 9892 & 12110 & 25972 & 4090 & 9929 & 12110 & 26129 \\
\hline & $\begin{array}{l}\text { as\% of } \\
\text { network }\end{array}$ & 15 & 38 & 47 & 100 & 16 & 38 & 46 & 100 \\
\hline \multirow{3}{*}{ Condition } & Standard & 44 & 17 & 24 & 1 & 21 & 27 & 29 & / \\
\hline & Mediocre & 36 & 68 & 0 & I & 37 & 70 & 6 & I \\
\hline & Bad & 20 & 15 & 76 & 1 & 42 & 3 & 65 & I \\
\hline \multirow{2}{*}{$\begin{array}{l}\text { Volume of } \\
\text { traffic }\end{array}$} & in Veh.km & 3878120 & 1273694 & 520730 & 5672544 & 4029428 & 1726157 & $\begin{array}{l}702 \\
380\end{array}$ & 6457965 \\
\hline & As\% & 68 & 22 & 10 & 100 & 62 & 27 & 11 & 100 \\
\hline
\end{tabular}

177. The limited extent of the paved network, and the poor state of the road system as a whole, pose a major obstacle for people in general, and for the poor in particular, in gaining access to basic services. The results of the ECAM show, in fact, that for people living in the countryside it takes on average an hour to reach a paved road, and that for the poor it takes longer than for the nonpoor.

178. An important lesson for formulating the strategy can be drawn from this analysis. Access to infrastructure services in Cameroon is much less satisfactory for rural than for urban dwellers. This factor may well be widening the economic gap between the cities and the countryside, which is home to half the country's total population, and more than two-thirds of its poor. The situation is explained in part by the fact that it is costly to provide infrastructure services to sparsely settled areas. It also reflects the relative weakness of rural people's influence on the budgetary process and of their ability to attract public spending on their behalf. Particular attention must therefore be paid to this situation when it comes to decentralizing public services, to ensure that some zones are not overlooked in favor of others, which would have the effect of fragmenting both the economy and society, and would constitute a major stumbling block to any effort to reduce poverty and inequality.

\footnotetext{
12 Traffic over rural roads is not given in the reference document: an absolute value was estimated as a fraction of traffic using dirt roads.
} 


\section{CHAPTER 3 : GROWTH AND POVERTY REDUCTION STRATEGY}

CInternational Monetary Fund. Not for Redistribution 


\subsection{VISION AND OBJECTIVES}

179. The government wants all stakeholders and beneficiaries to participate in the implementation of its poverty reduction policies and strategies, which draw on two sources of knowledge: on the one hand, a poverty profile built from a nationwide participatory consultation process and living standards surveys, and on the other, the lessons drawn from its long-standing efforts to carrying out macroeconomic, structural, and sector reforms. The central lesson from the analysis of poverty factors and trends is that economic growth, with its generation of many economic and revenue opportunities is essential to poverty reduction.

180. Cameroon's history is a case in point. Between 1996 and 2001, poverty incidence dropped by 13 points, of which 11 points were attributable to growth and only 2 points to redistributive policies. Hence, in developing its strategic approach, the government has endeavored to strike an appropriate balance between the competing requirements of economic performance and social development, with an overarching objective of reducing poverty.

181. The ultimate objective of the government's strategy is to achieve a sustainable and visible improvement in the standard of living of Cameroon's population that would come from tackling the very roots of poverty. This, the government believes, will result from implementing policies designed to create the conditions for strong, sustainable economic growth and from aligning its poverty reduction policies with the Millennium Development Goals (MDGs). More specifically, the government's targets for 2015 are to:

1. Eradicate extreme poverty and hunger, by halving the proportion of Cameroonians living below the poverty line and suffering from hunger;

2. Achieve universal primary education by ensuring that all children are able to complete primary education;

3. Promote gender equality and empower women, by eliminating gender disparities in primary and secondary education, if possible at all levels;

4. Reduce by two-thirds the mortality rate at birth and among children under 5;

5. Improve maternal health, by reducing the maternal mortality rate by three-quarters;

6. Combat and stop the spread of HIV/AIDS, control the incidence of malaria and other major diseases, and reverse the spread of these pandemics;

7. Ensure environmental sustainability by reducing by half the proportion of people without access to safe drinking water, achieve significant improvement in housing by integrating sustainable development principles in national policies, and reverse the current degradation of environmental resources; and

8. Create a global partnership to develop information and communications technologies and to implement policies and strategies that offer decent and productive work prospects to Cameroon's youth. 


\begin{tabular}{|c|c|c|c|c|c|}
\hline \multirow[t]{2}{*}{ MDGs } & \multirow[t]{2}{*}{ Monitoring Indicators } & \multicolumn{2}{|c|}{ Current status } & \multicolumn{2}{|c|}{ Goals and targets } \\
\hline & & Level & Year & Level & Year \\
\hline \multirow{4}{*}{$\begin{array}{l}\text { 1. Eradicate extreme } \\
\text { poverty and hunger }\end{array}$} & $\begin{array}{l}\text { 1. Proportion of population living } \\
\text { below the poverty line (poverty } \\
\text { incidence) }\end{array}$ & 40.2 & 2001 & 25.2 & 2015 \\
\hline & 2. Wasting rate among children & 6.0 & 1998 & 1.5 & 2015 \\
\hline & $\begin{array}{l}\text { 3. Malnutrition rate among } \\
\text { children (12-23 month) }\end{array}$ & 44 & 1998 & & 2015 \\
\hline & $\begin{array}{l}\text { 4. Underweight rate among } \\
\text { children }\end{array}$ & 22.2 & 1998 & & 2015 \\
\hline \multirow{3}{*}{$\begin{array}{l}\text { 2. Achieve universal } \\
\text { primary education }\end{array}$} & $\begin{array}{l}\text { 5. Net primary enrollment ratio } \\
\text { (6-11 years) }\end{array}$ & 75.2 & 2001 & 100 & \\
\hline & 6. Primary school access rate & 90 & 2000 & 100 & 2015 \\
\hline & 7. Primary school completion rate & 56 & 2003 & 100 & \\
\hline \multirow{4}{*}{$\begin{array}{l}\text { 3. Promote gender } \\
\text { equality }\end{array}$} & 8. Gender parity index & 85 & 2000 & 100 & 2015 \\
\hline & $\begin{array}{l}\text { Secondary education gender } \\
\text { parity index }\end{array}$ & 81.5 & 2000 & 100 & 2015 \\
\hline & $\begin{array}{l}\text { Secondary (general } \\
\text { education) }\end{array}$ & 87.5 & & 100 & \\
\hline & $\begin{array}{l}\text { Secondary (vocational } \\
\text { training.) }\end{array}$ & 61.7 & & 100 & \\
\hline $\begin{array}{l}\text { 4. Reduce child } \\
\text { mortality (under-five) }\end{array}$ & $\begin{array}{l}\text { 9. Under-five mortality rate per } \\
1,000 \text { live births }\end{array}$ & 150.7 & 1998 & 42.1 & 2015 \\
\hline \multirow{2}{*}{$\begin{array}{l}\text { 5. Improve maternal } \\
\text { health }\end{array}$} & $\begin{array}{l}10 . \text { Proportion of births attended } \\
\text { by skilled health personnel }\end{array}$ & 41.8 & 1998 & 70 & 2015 \\
\hline & $\begin{array}{l}\text { 11. Number of maternal deaths } \\
\text { for } 1,000 \text { live births }\end{array}$ & 430 & 1998 & 350 & 2015 \\
\hline \multirow{2}{*}{$\begin{array}{l}\text { 6. Combat HIV/AIDS, } \\
\text { malaria, and other } \\
\text { diseases }\end{array}$} & $\begin{array}{l}12 . \text { HIV prevalence among } \\
\text { sexually active population (ages } \\
15 \text { to } 49 \text { ) }\end{array}$ & 11.8 & 2002 & 9 & 2015 \\
\hline & $\begin{array}{l}\text { 13. Prevalence of malaria among } \\
\text { pregnant women and children } \\
\text { (ages } 0 \text { to } 5 \text { ) }\end{array}$ & 45.9 & 1997 & 25 & 2015 \\
\hline \multirow{3}{*}{$\begin{array}{l}\text { 7. Ensure environmental } \\
\text { sustainability }\end{array}$} & $\begin{array}{l}\text { 14. Proportion of land area } \\
\text { protected }\end{array}$ & 10 & 1997 & & 2015 \\
\hline & $\begin{array}{l}\text { 15. Proportion of population } \\
\text { without access to safe drinking } \\
\text { water }\end{array}$ & 49.5 & 2001 & 25 & 2015 \\
\hline & $\begin{array}{l}\text { 16. Proportion of population with } \\
\text { improved sanitation (flush } \\
\text { lavatory, improved pit latrine) }\end{array}$ & 42.8 & 2001 & & 2015 \\
\hline \multirow{3}{*}{$\begin{array}{l}\text { 8. Develop a global } \\
\text { partnership for } \\
\text { development }\end{array}$} & 17. Unemployment rate & 17.1 & 2001 & & 2015 \\
\hline & $\begin{array}{l}\text { 18. Telephone lines per } 1,000 \\
\text { people }\end{array}$ & 6.5 & 2000 & & 2015 \\
\hline & 19. Computers per 1,000 people & & & & 2015 \\
\hline
\end{tabular}

182. Table 1 above shows how much progress has already been achieved and provides a measure of the efforts still ahead to reach the Millennium Development Goals. In selecting its strategic priorities and actions, the government of Cameroon wishes to convey its political commitment to an ambitious but still feasible strategy toward economic growth and poverty reduction. In turn, implementing the strategy will require further efforts to diversify the economy and significantly accelerate real growth, and to put into action targeted measures designed to convert growth into greater poverty reduction. 
183. The growth and poverty reduction strategy proposed by the government is guided by seven clear-cut strategic priorities, each supported by a specific plan of actions. The government will periodically review the strategy in light of future changes in the environment, resource availability, and implementation progress. The strategic priorities are:

- Priority 1: Promotion of a stable macroeconomic framework;

- Priority 2: Strengthening growth through economic diversification;

- Priority 3: Empowering the private sector as the main engine of growth and a partner in social services delivery;

- Priority 4: Developing basic infrastructure and natural resources in an environmentally sustainable manner;

- Priority 5: Accelerating regional integration within the CEMAC framework;

- Priority 6: Strengthening human resources, bolstering the social sector, and promoting the integration of vulnerable groups into the economy;

- Priority 7: Improving the institutional framework, administrative management, and governance.

\subsection{A MACROECONOMIC FRAMEWORK THAT CREATES THE CONDITIONS FOR GROWTH}

184. Maintaining a sound macroeconomic framework is the cornerstone of any growth and poverty reduction strategy. The government is committed to pursuing a prudent, two-pronged policy approach: maintaining macroeconomic equilibrium while creating an enabling environment for growth and private sector development; and allocating appropriate budgetary resources to priority sectors, ensuring actual progress toward poverty reduction and economic growth. In the short term, the two components may at times conflict, but they complement one another in the medium term. Budget policies that accord priority to social sector expenditures may at times be at odds with fiscal stability, increasing debt and debt service and reducing the government's fiscal capacity to maintain this policy course in the medium term. However, insufficient or poorly targeted budgetary expenditures can lead to underinvestment in priority sectors and infrastructure, with an overall negative impact on both growth and poverty reduction.

185. Macroeconomic stability fosters growth and welfare improvement in the medium term. It alleviates the burdens of debt, inflation, and high interest rates that penalize all economic actors and more particularly the poorest households. It reduces the level of uncertainty and country risks and hence decreases the cost of capital. It contributes to maintaining a stable real exchange rate. The latter three factors help improve overall economic competitiveness and foster investment, production, and export diversification, thereby accelerating growth, reducing the volatility of the economy, and maximizing welfare.

186. In this context, satisfactory implementation of the government's two latest economic and financial programs helped the economy return to a stable 4.7 percent growth between 1996 and 2001, with a moderate 3 percent inflation. The implementation of tax and budget reforms has also contributed to putting public finances in order and initiating a debt burden reduction process. Furthermore, domestic saving and investment have also improved as a result of the structural reforms.

187. Despite this progress, developments in 2002 indicate that improvements remain weak. The 4.2 percent estimated growth rate is lower than over the preceding 5 years. Also, the 
annual inflation rate fluctuated between 4.5 percent at end-June 2002 and 2.8 percent, as against 1.3 percent in 2001, mainly owing to variations in food prices; and the current account balance has begun to deteriorate (-0.8 percent of GDP) after being positive for two consecutive years.

188. These developments show that insufficient growth and lack of a solid growth foundation can also lead to macroeconomic disequilibrium. For example, despite a strict monetary policy, inflation could arise if supplies are unable to meet minimal levels of demand (for example, food crops in recent years in Cameroon); public finances could deteriorate as a result of losses in tax receipts due to income stagnation or contraction. In the medium term, low growth is insufficient to create the level of saving and investment needed to diversify the economy. Of course, it is possible to maintain growth driven by primary products (oil, agricultural products) with a relatively low level of investment, as has been the case in Cameroon during the last decade as a result of the rapid expansion of production in the primary sectors (food crops), and growth can be driven in the informal sector through labor-intensive activities and without new investments. In contrast, industrial diversification will require substantial investment and, therefore, a domestic saving and investment rate in excess of 25 percent of GDP. In the absence of diversification, the population will remain vulnerable to unexpected climatic or economic changes, making it harder to avert poverty.

189. The government is cognizant of the need to accelerate growth, strengthen the social sector, and consolidate macroeconomic stability. Therefore, it is determined to redouble its efforts to deepen ongoing structural reforms and to mobilize the population and development partners behind an ambitious yet realistic and sustainable program, aiming to progressively accelerate growth in the medium term while maintaining prudent tax and budgetary policies.

190. The government intends to achieve its objectives by according priority to developing the non-oil sector by diversifying production and exports, and improving tax and customs revenues. This will ensure that an appropriate framework for rapid expansion of the private sector is in place and that the privatization program is vigorously pursued, including the state divestiture from major agroindustries and the concession contracts with private operators for large utilities (railroad, electricity). Within the CAEMC, such increased diversification of the economy is consistent with increased regional integration.

\subsection{CONSOLIDATING GROWTH BY DIVERSIFYING THE ECONOMY}

191. The government understands that macroeconomic reforms alone will not suffice to diversify the economy and significantly reduce its dependence on the primary sector (agriculture and mining), which is vulnerable to changes in natural conditions and in the world market prices of its exports. Moreover, oil, which has greatly contributed to growth and public finances, has been declining for several years owing to the depletion of oil reserves. Therefore, increased diversification of the non-oil sector in the medium term is essential for Cameroon. With such a diversification, growth would return to the 6-7 percent range while revenue would be less volatile, making it possible to maximize the impact of growth in terms of poverty reduction and improvement of the population's welfare.

192. This explains why the government has launched studies on the prospects for economic diversification in order to identify potential sources of growth. The preliminary findings indicate that: (i) Cameroon's economy will rely on agriculture to maintain a growth level one point higher than the 1997-2001 level, contributing to poverty reduction, particularly in rural 
areas; and (ii) Cameroon has noticeably competitive assets in the industrial area that could be developed through appropriate policies and well-targeted measures.

\section{Box 8. Studies of Competitiveness and Sources of Growth in Cameroon's Economy}

Two large studies have been initiated by the government to analyze the competitiveness of the economy and identify potential sources of growth to reduce poverty. While the work is ongoing, preliminary findings have been used to strengthen the growth strategy as set forth in the PRSP.

\section{Study of the sources of growth to reduce Cameroon's poverty}

This study intends to identify potential sources of growth outside the oil sector and to recommend development policies. Studies have been conducted by a group of Cameroon's experts consisting of academic and private sector consultants. The following themes have been studied:

(i) Agricultural subsector;

(ii) Industrial and commercial subsector competitiveness;

(iii) Tourism;

(iv) Private sector development;

(v) Human capital;

(vi) Financial sector;

(vii) Infrastructure-power, telecommunications, and transportation;

(viii) Economic and trade policies.

A summary report is currently being prepared and will be available within the next few months.

Source: "Etude sur les sources de la croissance pour la réduction de la pauvreté au Cameroun," BDS, 2002.

\section{Diagnostic study on the competitiveness of Cameroon's economy}

This study, sponsored by the competitiveness committee, has been financed by GTZ. It is aimed at evaluating competitiveness by analyzing Cameroon's economic framework as well as specific subsector issues. Specifically, the study expects to:

- Provide an overall picture of the competitiveness of Cameroon's economy;

- Highlight the development and growth potential of productive sectors and subsectors, and identify the sectors and subsectors with high potential for contributing to reducing poverty and enhancing competitiveness.

An interim report is available, and work continues to finalize the study.

Source: Competitiveness committee.

\subsubsection{Rural sector: a key sector for economic growth}

193. Cameroon's economy continues to be dominated by the rural sector through its contribution to growth as well as its large poverty reduction potential. According to projections, about half of the country's population lives in rural areas and is employed in agricultural, pastoral, or forestry activities. The poverty profile shows that Cameroon's poverty is primarily rural. 


\section{Box 9. An Integrated Rural Development Strategy}

The government aims at promoting a strong, sustainable, and equitable agricultural sector growth that: (i) reduces poverty; (ii) addresses the needs of a growing demand for food crops at the national level; (iii) integrates the global and subregional markets; and (iv) sustains the sector's performance over time. It has drafted an integrated rural development strategy with four strategic priorities: modernizing production facilities, restructuring the institutional framework, enhancing incentives, and sustainably managing natural resources.

Modernizing production facilities involves: (i) improving access to, and the availability of, production factors such as land, water, and farm inputs for the neediest of the rural population; (ii) promoting access to innovative technology with a stronger link between agricultural research and extension; and (iii) enhancing the competitiveness of the productive subsectors. Emphasis will be placed on small holders while attention will be given to developing small- and medium-scale agricultural enterprises.

The government will encourage the establishment of consultation mechanisms and the development of contractual relationships between input supply companies and agricultural professional and interprofessional organizations. Similarly, the government will facilitate the emergence of a more effective private sector and NGOs that could effectively take over outreach and education activities, rural community organizations, input supplies, veterinary services, seed and plant production, support for trade activities, etc.

Restructuring the institutional framework is intended to promote greater professionalism in the rural sector, including farmers, ranchers, and fishermen, through capacity building. In the forestry subsector, priority actions include carrying out reforms and implementing the results of the institutional review, as well as implementing the forestry environment sector program, which is a key pillar of the government's forestry and environmental policy. In the agricultural and livestock subsectors, measures will be carried out to improve the quality of public service delivery and to redirect policies and institutions toward the needs of the rural poor.

Enhancing incentives will allow the government to: (i) establish, in partnership with the private sector, conditions for more efficient market mechanisms so as to improve access by rural producers to new technology and other requirements, including from the financial sector; and (ii) put into effect and enforce the existing sector regulations.

Sustainable natural resource management will help the government organize and encourage private and community initiatives favoring environmentally friendly development by: (i) coordinating the management of renewable natural resources, involving all administrations and key users (NGOs), civil society, rural communities, etc.; (ii) restoring and safeguarding production potential; and (iii) ensuring reliable local management of rural community infrastructures. All measures will be designed to sustain natural resources, focusing particularly on protecting soil fertility, the conservation of water resources, the conservation of land pasture, and the conservation of biodiversity, etc.

The strategy adopted for the rural sector is a "proactive" growth promotion strategy built on: (i) smallholder agriculture; (ii) semi- and intensive farming; (iii) processing and marketing units of products and subproducts; and (iv) the specific regional agroecological features. The strategy is aimed at implementing active socioeconomic policies to ensure that the benefits of growth are shared. The policy includes rural infrastructure development activities (building village-level warehouses, markets, rural and feeder roads, access to safe drinking water and electricity, etc.) and community development. Emphasis will be placed on a gender-sensitive as well as participatory approach.

Source: MINAGRI/MINEPIA/MINEF.

194. During the 1970s and 1980s, rural sector performance was very weak given the vast potential of the sector. Hence, Cameroon has continued to import cereals to meet the needs of its population. Between 1971 and 1998, imports increased by a factor of 10 from 32,100 metric tons to 348,148 metric tons. Imports per capita increased from $7.2 \mathrm{~kg}$ to $24 \mathrm{~kg}$ during the same period, while cereal production per capita declined from $157 \mathrm{~kg}$ to $85 \mathrm{~kg}$. The deterioration of the productive environment (changes in ecosystems, declining soil fertility, etc.) has threatened the relevance of the current production system. Similarly, the sector is facing cross-sector constraints, particularly a lack of rural infrastructure, inadequate dissemination of economic information, as well as reduced productive capacity. During the 
last decade, the supply response in agriculture has generally been unable to respond to market signals or meet the needs of rural households.

195. The government's integrated rural development strategy will be implemented through several programs highlighting five priority areas: (i) local development; (ii) production development; (iii) institutional support; (iv) sustainable management of natural resources; and (v) financing mechanisms available to the rural sector. The government will also integrate into its rural development strategy cross-sector policies and actions related to land management reform and capacity building.

196. The strategy with regard to the local development priority will endeavor to: (i) strengthen community and commune ability to address local development issues; (ii) support infrastructure construction and the removal of specific constraints at the local level; (iii) develop income-generating activities; and (iv) ensure the active participation of local stakeholders in developing their communities through progressive decentralization. The National Participatory Development Program (PNDP), the Community Development Support Program (PADC), and the RUMPI project constitute the prime operational frameworks.

197. The production development component will focus on: (i) supporting farming development and a better integration of production activities (crop, livestock, and agroforestry production); (ii) developing high-potential subsectors; (iii) promoting the emergence and development of professional and interprofessional organizations; (iv) supporting applied research and demand-driven extension services so as to alleviate priority production and farming constraints; and (v) agricultural product processing.

198. Institutional support and renovation of vocational and professional training will help those in the rural sector flourish in their new environment. Key measures will involve building capacity, allowing: (i) administration to focus on key public functions and provide essential services (training, information, statistics, etc.); (ii) economic operators to improve their competitiveness; and (iii) professional organizations to improve their negotiation capacity and to become valid decision-makers in designing and implementing the strategy.

199. Sustainable natural resource management will be achieved through the forestry/environment sector program, which is the key operational framework (see paragraph 3.3.1.3).

200. Rural sector financing includes two subcomponents. The first (the National Microfinance Program) aims at improving the rural population's access to microfinance institutions and at strengthening the capacity of these institutions. The second plans to implement mechanisms relating to medium and long-term financing for farmers and agriculture-based business investments. 


\section{Box 10.The National Participatory Development Program (PNDP)}

As an expression of its commitment to significantly reduce poverty by 2015 , the government has formulated, with assistance from the international community, the National Participatory Development Program (PNDP). This program, within the context of progressive decentralization, will help the government formulate a mechanism to hold grassroots and public decentralized structures more accountable and get them more involved in their own development.

The PNDP is consistent with the integrated rural development strategy and attempts to create synergies for a functional partnership between grassroots communities, the state, civil society, NGOs, and donors. It is intended to promote an equitable, efficient, and sustainable development of the rural population. Its primary beneficiaries are grassroots communities and/or organizations, neighborhoods, local governments, and other community associations, etc.

The 15-year program will be deployed nationwide in three phases. Its four components are: (i) a rural community development support fund; (ii) support to communes in the context of progressive decentralization; (iii) building local capacity; and (iv) monitoring, evaluating, and communication.

The Rural Community Development Support Fund (FADCR) will cofinance microprojects and activities initiated by neighborhoods, village communities, and other civil society members, on the basis of "commune development plans" drafted in a participatory approach and approved by a communal joint community.

Support of the progressive decentralization of communes aims at preparing communes, institutions, and grassroots communities to better integrate progressive decentralization and poverty reduction in rural areas.

Building local capacity will try to improve the knowledge and skills of development participants in order to make their joint cooperation in poverty reduction efforts more effective. Potential targets of this capacity building exercise are grassroots communities, decentralized and local governments, deconcentrated government agencies, NGOs and service providers, microfinance institutions, etc.

The monitoring/evaluation and communication component will provide development participants with the information and decision-support/management tools needed to accomplish their goals.

Source: MINEPAT.

\subsubsection{Agriculture}

201. Following the construction of major roads to the Eastern and Southern provinces, marketing prospects for starch crops, cereals, fruits, and vegetables appear good in the short and medium term, particularly for plantain, potatoes, onions, and cassava. The support of household farming will be provided by the development of agricultural activities in urban areas. The goal is to foster the emergence of intensive, land-saving, and highly efficient production systems, to provide adequate supplies of local products to urban areas, particularly in fruits and vegetables. Such intensive production systems would offer employment opportunities to a large number of people, currently underutilized, and would therefore increase production.

202. Starch crops. The government's policy is to increase the consumption of fresh and processed food in order to reduce food imports and improve national food security. The main strategic objectives are to: (i) improve farm productivity (enhancing the use of quality seeds and technology through extension services); (ii) market starch crops (stabilize urban supply and pricing); and (iii) promote export- or processing-oriented SMEs/SMIs. A special cassava program will be established because of the product's social and economic importance (a third of cassava production is processed). In addition to meeting the requirements for fresh and processed products, the program will take into account subregional demands and national 
industry requirements for animal food, as well as starch production (see Annex 3.1 for more details on the cassava subsector).

203. Cereals. The national cereal policy aims, among other things, to: (i) consolidate cereal production in current production systems so as to increase food security and raise farmers' incomes; and (ii) promote production, particularly in areas where competitiveness in domestic and subregional markets is good (see Annex 3.1 for more details on corn, millet, and sorghum production).

\section{Box 11.The Marketing Cost of Agricultural Products: Food Crops}

\section{- Issues}

The marketing of food crops incurs many costs (transportation, handling, warehousing, losses). Beyond a certain level, a cost increase would reduce availability and hurt the crop's competitiveness, even if it enjoys a comparative advantage. Therefore, controlling cost is imperative to improving the consumers' as well as the producers' welfare.

\section{- Methodology}

The cost of marketing food crops can be analyzed in terms of its percentage relationship to the total production cost and in terms of the gap between producer prices and retail prices. The gap illustrates the relative share of intermediaries and their profit margins in the marketing system.

\section{- $\quad$ Results}

Intermediaries' margins constitute the single most important source of cost increase. Transportation is second. For some products, such as potatoes, the intermediaries' profit margin is more than 100 percent of producer prices. Transport cost depends on the distance and the season (rainy or dry season). In theory, the cost per kilometer ranges between CFAF 202 on average for a small truck and CFAF 239 on average for a large truck. Estimates in the table below are based on dry season costs, except for potatoes. The destination market is Douala for plantain and Yaoundé for corn and potatoes.

\begin{tabular}{lrrr}
\hline Marketing cost for selected food crops & & \\
\hline & Plantain & Corn & Potatoes \\
\hline Marketing cost as \% of total cost & 31 & 44 & 43 \\
Of which transport cost & 10.2 & 10.1 & 19.5 \\
(Sales price - producer price): producer price (in \%) & 140 & 104 & 200 \\
Intermediaries' margin in \% of producer price & 96 & 26 & 126 \\
\hline
\end{tabular}

The table above indicates that intermediaries (wholesalers, merchants, retailers, carriers) are the primary beneficiaries of food crop marketing. Better-organized producers could decrease the number of intermediaries, thereby increasing their incomes. Maintaining feeder roads is also another important factor.

Source: Study on improving the marketing and competitiveness of Cameroon's agricultural products, Nicolas Gergely, April 2002.

204. The fruit and vegetable subsector holds promise in terms of production and export diversification. Subsector measures include: (i) making better use of domestic and subregional urban markets; and (ii) designing, initiating, and implementing specific development support programs for priority subsectors, such as pineapple, plantain, potatoes, gum Arabic, and onion. Emphasis will be placed on (i) facilitating trade in and exports of fresh products by opening up landlocked production areas; (ii) supporting the organization of wholesale marketing in primary harvesting areas, major trade centers, and border markets; and (iii) improving packaging structures (see Annex 3.1 on potatoes). 


\section{Box 12. Comparative Advantages of Agricultural Subsectors}

\section{- Issues}

The agricultural sector contributes about 40 percent of GDP, represents 21 percent of total exports, and employs a large share of the population. Agricultural competitiveness and growth potential are central issues in the poverty reduction strategy. Which subsectors with comparative advantages would benefit from the implementation of supporting measures to increase production and achieve sustainable growth?

\section{- $\quad$ Methodology}

A microeconomic study on agriculture subsector competitiveness has shed some light on this question. The study focused on Cameroon's key marketed products, including basic food crops: plantain, cassava, corn, and potatoes, poultry from the animal subsectors, and cocoa and coffee. The study calculated the economic return of the production in each subsector. The economic return is seen as a comparative advantage indicator (the domestic resource cost coefficient or "DRC"). Assuming all national production is either exportable or can replace imports to generate or save foreign exchange, DRC measures the cost of gaining or saving foreign exchange through domestic production. When the cost is less than the international prices, the DRC is less than one and the national economy enjoys a comparative advantage.

\section{- $\quad$ Results}

The figures in the following table show encouraging prospects for most of Cameroon's agricultural subsectors. In general, regarding food crops, activities are economically profitable with the exception of intensive or semi-intensive aviculture with the purchase of provender. Simple arithmetic has been used to estimate the average DRC for each subsector. DRCs have been calculated for two or three geographical areas, but because of a lack of data, it has not been estimated for cassava and potatoes: the Ebolowa area (extensive model), the Southwest (semi-intensive model), and Mbam and Lékié (intensive model). Concerning Arabica coffee, an extensive model whereby coffee is intercropped with food crop cultivation, and a semi-intensive model have been used to evaluate subsector performance. The cultivation model for Robusta is typically semi-intensive. Available data have made it possible to calculate the DRC for each subsector as well as the equilibrium producer price. The results show that, while cocoa/coffee subsector activities are economically profitable given that all the DRCs are less than one, in contrast the financial return shows tremendous variation in international prices.

\section{Competitiveness of selected basic food crops}

\begin{tabular}{|c|c|c|c|c|c|c|c|}
\hline Product & Plantain & Corn & & & cultur & & \\
\hline & & & 1 & 2 & 3 & 4 & 5 \\
\hline DRC & 0.58 & 0.50 & 0.65 & 1.1 & 0.95 & 0.8 & 0.70 \\
\hline Marketing cost in $\%$ of total cost & 39 & 51 & - & - & - & - & - \\
\hline
\end{tabular}

\begin{tabular}{lcccccc}
\hline Competitiveness of selected export crops & & & \\
\hline \\
\hline Product & Cocoa & & & \multicolumn{2}{c}{ Coffee } \\
\cline { 2 - 7 } & & & & Robusta & Arabica \\
\cline { 2 - 7 } & Ebolowa & Southwest & Mbam-Lékié & & 1 & 2 \\
\hline DRC & 0,2 & 0.2 & 0.2 & 0.9 & 0.6 & 0.4 \\
Producer price at equilibrium $/ \mathrm{kg}$ & 202 & 227 & 296 & 199 & 423 & 331 \\
\hline
\end{tabular}

Note: With respect to aviculture: (1) denotes intensive or semi-intensive aviculture, including the production of provender; (2) denotes intensive or semi-intensive aviculture with purchase of provender; (3) denotes traditional aviculture; (4) denotes improved traditional aviculture; and (5) denotes intensive egg production.

\section{- $\quad$ Implications for the rural development strategy}

High marketing costs, as mentioned in the preceding box, weigh down highly perishable food crops, resulting in lower producer prices. Improving producer incomes will depend on creating appropriate feeder roads, promoting producer organizations, and improving information. These are the major supports for the sector in the rural strategy and the PRSP.

Source: Study on improving the marketing and competitiveness of Cameroon's agricultural products, Nicolas Gergely, April 2002.

205. Traditional export crops will still play a major role in the economy of rural areas. For subsectors such as cotton, coffee and cocoa, and palm oil, where family farming plays an important role, key recommendations include:

Strengthening the capacity of producer organizations to improve production operations, marketing, and bringing products up to market standards; 
- Improving subsector economic performance in order to increase producer incomes and improve the ratio of competitiveness/cost for major domestic and external markets (see box);

- $\quad$ Consolidating production systems while maintaining their diversity in order to better manage market-related risks. This includes support for producer initiative, as has been done for palm oil in low altitude forest areas, corn in the cotton producing area and central savanna, and fresh vegetables and tubers in most regions.

206. Other promising subsectors. The preliminary results from the sources of growth study highlighted Cameroon's natural propensity for the development of new, promising growth subsectors, such as pepper, horticulture, watermelon, and organic farming. Development prospects for such activities exist in forest areas of the Littoral and Southwest provinces, and in the highland area of the West and Northwest provinces. Additionally, these areas are close to Douala, the main entry and exit port of the country, which is an important asset for export opportunities.

\subsubsection{Livestock, fisheries, and commercial aquaculture}

207. In addition to their important role in improving food security, livestock and fisheries also contribute to the creation of wealth, thanks to the employment they generate and other related activities, including: (i) production of organic fertilizers to improve agricultural yields; (ii) animal traction which improves labor productivity; and (iii) transportation of agricultural products, particularly from landlocked areas to collecting centers or to neighboring markets. The government's strategy concerning livestock and fisheries is also geared toward improving productivity and competitiveness in this subsector, including ruminants, short-cycle cattle farming, semi-intensive cattle farming, nontraditional cattle farming, fisheries, and commercial aquaculture.

208. Ruminants. In pastoral and agropastoral areas, programs will endeavor to: (i) improve the management of pasture lands (Hurum) and of conflicts therein; (ii) train and organize cattle ranchers and agroranchers; (iii) develop cattle fattening or dairy producing activities, particularly in suburban areas of mid-sized towns (Bamenda, Garoua, Maroua, Ngaoundere, etc.); and (iv) improve dairy farm sanitation. Also, the development of producers' organizations and professionalism will be emphasized in order to better integrate the poorest ranchers and agroranchers into the dynamics of agricultural growth.

209. Cattle fattening and processing. This activity is currently being developed around major cities in the Northern part of the country, using cottonseed oil and fodder. Promoting cattle fattening will be the objective of a program especially targeting women and youth to help reduce poverty. The government's strategy for this area will include: (i) restructuring of the cattle subsector; and (ii) promoting a supply chain bringing together extensive breeding activities, animal fattening, and meat processing (canning). These will be consolidated through production contracts, making transactions safer between different levels of the subsector. Renewed economic viability of the subsector would lead to an improvement in livestock productivity per capita.

210. Periurban milk farming. This activity is being developed around major towns in the North and in Bamenda, with the development of a semi-industrial and a traditional marketing sector in urban markets (Maroua, Garoua, Ngaoundere, Bamenda). Small-scale ranchers, and 
very often women living near urban centers, are the key players. In order to ensure economic sustainability, government action will focus on structuring the subsector in order to improve production, processing, and marketing.

211. Short-cycle animal husbandry and village-level stock farming. Aviculture and pig farming are activities usually carried out by women. They play an important role in the nutrition, incomes, and savings of the poorest households in rural and urban areas, and therefore must be supported in the poverty reduction and food security strategy. A particular emphasis will be placed on types of stock farming with very low production costs. Activities are intended to decrease mortality by providing better access to a prophylactic system through a network of village-level female vaccinating agents, and to improve product marketing through producer organizations operating within the subsector.

212. Semi-intensive stock farming. This type of stock farming has growth potential, given the consistent increase in the urban population and their protein needs. Nevertheless, production costs are still too high, making it difficult to compete with imports. This gap could be bridged with a better organization of producers (health coverage, group purchase of inputs, group marketing, etc.). The government will promote professional and interprofessional structures to facilitate consultation and negotiation between the various levels of the subsector.

213. Nontraditional stock farming. The demand for nontraditional stock farming products remains very high in domestic and regional markets. Game ranching would decrease the capture of wild animals, contributing to the conservation of ecosystems. Activities will include improving domestication techniques for a better and more sustainable management of species.

214. Regarding the progressive retrenchment of the state from the productive sector, the government intends to establish consultation mechanisms to: (i) foster the emergence of joint trade organizations; and (ii) define an appropriate institutional and regulatory framework. Its objectives are to: (i) train and structure producers to improve the productivity of their stock in the context of the national agricultural extension and research program (PNVRA); and (ii) improve the accessibility of veterinary equipment and medicine, facilitating the development of private customers for veterinarians.

215. Fisheries. Cameroon has some comparative advantage in the area of industrial and artisanal fisheries. The latter have good growth potential that could benefit the poor population. However, since 1997, industrial and artisanal fisheries have followed different development patterns. While the modern sector has grown substantially, the production of artisanal fishery has been mostly stagnant. The population's food requirements in the subregion, as well as the needs of agroindustry, provide growth prospects as regards the demand for fishery products. Cameroon's coastal areas are particularly favorable to the development of fisheries for crustaceans and other high-value species, for which the demand should continue to grow both domestically and in the subregion.

216. However, several constraints hamper the development of this subsector, particularly: (i) low professionalism in the artisanal subsector; (ii) high cost of acquiring fishing equipment, which is a serious handicap for local formal sector operators; and (iii) difficulties in the transportation and conservation of products. 
217. To foster development in the subsector, the government plans to: (i) improve productivity in artisanal fishery through better organization of fishermen, training, counseling for young fishermen, and facilitating their access to appropriate fishing equipment; and (ii) ensure satisfactory fishing in national waters based on performance contracts signed between the government and the Maritime Artisanal Fishing Development Mission.

218. Commercial aquaculture. While it was once a dynamic activity, aquaculture has declined as a result of technical and organizational problems. The national market offers enormous opportunities, as fish is still the single largest source of animal protein and accounts for the highest import volume. The key issue is to improve the production of young fish with technical solutions, allowing producer groups to become autonomous and to disseminate improved production practices among farmers. The government's strategy aims to provide as many aquaculture producers as possible with the opportunity to become small businesses and rapidly increase their production and incomes by introducing new and more productive species. The number and dynamism of these small trade-oriented aquaculture enterprises will present, in favorable agroecological areas, the opportunity for integrated development based on a large number of independent producers.

\subsubsection{Forestry}

219. In recent years, the government has carried out major reforms aimed at increasing the forestry subsector's contribution to GDP while ensuring the sustainable development of forestry products and protecting other natural ecosystems. The reforms have been fruitful, leading to: (i) a restructuring of the local industry; (ii) high job creation; (iii) an improvement in tax revenue, a portion of which is redistributed to communes and rural communities; and (iv) a more transparent logging rights award system involving the participation of independent observers in logging rights commissions.

220. The 1994 Forest Law and subsequent forest sector reforms have improved the welfare of the forest population, which now receives part of the annual forestry tax (RFA). This group also benefits from the social welfare activities undertaken by the logging companies (in the case of large multiyear logging concessions). The goal of these reforms is to have forestry become: (i) a key sector in poverty reduction; and (ii) a major area for Cameroon's industrialization and exports.

221. Community forestry is of great relevance to the generation of income for the rural population. To sustain it, the government, with the support of its partners, has issued regulations that facilitate access to and direct community involvement in forest production activities. The government wants to further develop community forestry, helping local communities to become long-term rights holders and managers of forest and wild life resources. Community forestry objectives are aimed at: (i) generating income at the individual, group and community level; (ii) ensuring the sustainability of income streams and the forest resource base through simple management plans; and (iii) ensuring that communities feel empowered through training and employment measures.

222. However, because of persistent subsector problems, including illegal logging and weak enforcement of the minimal logging diameter (DME), forestry activities have not yet benefited from the full impact of the recent reforms. In order to improve the sector's economic and environmental performance, the government will ensure the full implementation of the provisions set forth in the Yaoundé Declaration concerning sustainable 
conservation and management of tropical forests, and in the Forest Emergency Action Plan and the reforms introduced under the third structural adjustment credit (SAC III), including:

- securing priority access by local people to forests for community-based forest management;

- creating a forestry stabilization fund to ensure the efficient and transparent redistribution of forest revenue, including forestry taxes, to local communities;

- making management plans mandatory for all production forests and supporting these measures through: (i) transparency in the awarding of further forest concession contracts and the enforcement of logging regulations; (ii) enhanced controls; and (iii) more stringent enforcement of penalties;

- combating the illegal use of forestry resources;

- the preservation and stability of the forest ecosystem, including the development of protected areas;

- implementing regulations relative to nontimber forestry products (NTFPs); and making sure that the new Forest and Environment Sector Program (PSFE) is effectively implemented. The objective of this program is to help the public and private sectors establish better and more sustainable management of forestry and wildlife resources from an ecological and socioeconomic perspective, and to link good sector governance to poverty reduction goals.

223. The government intends to promote high value-added forest industries as a means of creating wealth, jobs, and new markets, both domestically and abroad. The strengthening of downstream support capacities in the wood subsector, involving many small-scale cabinetmaking and woodworking enterprises, will have a positive economic impact on many households, whose incomes depend on this activity. Support to small and informal woodbased enterprise is envisioned in the form of training in technical and management skills, as well as product promotion.

\section{Box 13. The Forestry Environment Sector Program (PSFE)}

The PSFE is a national sector development program prepared by the government and open to financing from all donors as well as to contributions from civil society and NGOs. It aims to create a consistent framework for all interventions made with a view to achieving the national forestry and wildlife policy objectives.

Through the PSFE, the government is seeking to introduce a management information system to effectively monitor and control forestry and environmental activities, by bringing together disparate efforts under various projects and ensuring that all projects are consistent with the country's development objectives.

The PSFE, which will be implemented over 10 years, has six main components: (i) knowledge of research and ecological monitoring; (ii) the development of production forest from state domains, and enhancing the value of forestry products; (iii) preservation of biodiversity and increasing the value of wildlife products; (iv) community management of forestry and wildlife resources; (v) environmental management of development operations; and (vi) institutional strengthening, training, and research.

The PSFE takes into account and creates a consistent framework for a large number of pre-existing activities and projects, including those coming from the National Forestry Action Plan (PASN), the National Environmental Management Plan (PNGE), and the Emergency Action Plan (PAU). The promotion of reforestation and the management of woodlands are covered by specific PSFE subprograms which include: (i) revitalizing the promotion of tree planting under the National Forestry Development Agency (ANAFOR); and (ii) creating fuel wood master plans to supply urban centers in the Northern part of the country.

Source: MINEF. 


\section{Box 14.SAC III Forestry Reform}

For several years, Cameroon's government has been engaged in efforts to improve the management of forestry resources and make them more sustainable. Accordingly, it has carried out important reforms that are regarded as pioneering efforts in the central African subregion. These reforms include:

- The economic and financial audits of the forestry subsector (January 2000). Its recommendations, approved by the government, are included in the July 2000 national budget;

- The publication of regulations concerning the design, approval, monitoring, and control of management plans for production (May 2001);

- Establishing a guarantee system to ensure proper execution of management plans (November 2001); regulations concerned include the parameters necessary to calculate taxes to be paid, and the modalities for control and collection of forest fees;

- The publication of a regulation giving local communities priority over industrial companies when exercising forest exploitation and management rights over lands located in the proximity of villages (December 2001). This regulation describes the modalities to be followed for a community to acquire the rights and to manage a community forest;

- $\quad$ Transforming the National Forestry Development Office (ONADEF) into to National Forestry Development Support Agency (ANAFOR) in June 2002;

- Signing of two conventions with well known international institutions (Global Witness and Global Forest Watch/World Resource Institute) to help supervise the implementation of forest management plans, introduce remote sensing, and provide support for MINEF's field control ;

- $\quad$ Implementing the Secure Forestry Revenue Program (PSRF).

The focus of the forest sector reform in the coming years will be on maintaining independent observers with the commissions presiding over the competitive award of concessions, on maintaining the Independent Observers supporting MINEF in field inspections, as well as on the full implementation of the measures recommended by the forest sector institutional review. However, the government understands that the application of the above reforms remains insufficient and is committed to further improvements leading toward greater transparency in sector activities.

\section{Source: MINEF.}

\subsubsection{Artisanal mining}

224. Despite its potential, mining activity is essentially artisanal. It focuses primarily on gold, diamonds, sapphire, clay, natron, sand, rutile, and building and ornamental stones, etc. At the beginning of the $20^{\text {th }}$ century, artisanal mining contributed about 20 percent of the national economy. Available statistics at the end of 2002 showed that about 30,000 people still work in the subsector, marketing most of their products illegally. Artisanal mining suffers from a lack of skilled manpower, a lack of interest by economic operators, the lack of a technical assistance strategy, the absence of mining artisans' organizations, and a lack of access to microcredit. However, better and more sustained support could help the subsector realize its growth potential.

225. The new mining code established in the context of the subsector development lays the foundation for a new artisanal miner, defining him/her legally as a small-scale businessman engaged in formally marketing mining products. To achieve this objective, the national artisanal mining organization and promotion strategy includes: (i) strengthening institutional capacity; (ii) organizing artisans into professional organizations; (iii) increasing access to technical facilities and information; (iv) improving access to microfinance and microcredit; (v) facilitating and consolidating the marketing system; (vi) offering services to mining communities; and (vii) protecting the environment. 


\subsubsection{Industrialization to enhance and stabilize growth}

226. Until the crisis of the mid-1980s, the industrial sector had evolved into an environment protected by tariff and nontariff barriers, resulting in excessive manufacturing costs. The high cost of power, transportation, and telecommunications, the inadequate infrastructure, and the low productivity of human resources have considerably reduced the competitiveness of Cameroon's industry, despite tariff barriers or quotas, compared to products of its competitors or imported substitutes. The adjustment measures, such as the progressive liberalization of trade and of product as well as input prices have failed to trigger automatic adjustment in the industrial sector. The protectionist measures benefiting these industries in the past have not favored the development of a globally competitive industrial and technological base.

227. The government gives top priority to the industrial sector, which it regards as the key source of poverty reducing growth in the medium term. The public sector has the ambition of minimum annual growth of 6 to 7 percent, possibly reducing poverty by 50 percent by 2015 . This objective can be achieved only with an increased contribution to the national product from industry and services. Furthermore, industrial development will have important spillover effects on agriculture and services, investment and high value-added exports. Moreover, it would offer outsourcing opportunities to the SME/SMI sectors, favoring the development of interindustry links as well as greater integration of the industrial network. Beyond the direct effect of creating jobs and generating revenues, spillover effects on agriculture and services would also contribute to improving the incomes of the poor.

228. Cameroon has some competitive advantages in the manufacturing industry: (i) the privileged geographical position of the country in the Gulf of Guinea, given a potential market of 200 million consumers in Central Africa, including Nigeria; (ii) the availability of a wide range of raw materials and skilled manpower; and (iii) the potential for developing core physical infrastructures (hydropower potential, road network, telecommunications, urban development), as well as financial, human, and institutional resources and making them available to manufacturing industry.

229. Yet the industrial sector's performance remains well below its potential, as it is, like the other productive sectors, constrained by the lack of an appropriate transportation, power, and telecommunications infrastructure, etc. These constraints are analyzed in detail in the private sector section. More specifically, structural weaknesses also restrain the development of Cameroon's industry and reduce its role in the economy. These weaknesses include: (i) the lack of a selective support policy globally promoting the manufacturing capacities of high potential subsectors in areas such as product design and development, the development of appropriate skills, technological expertise, export promotion, outsourcing to SMEs, access to financing, etc.; and (ii) weak vertical integration, with most inputs for the industrial sector, such as raw materials, intermediate products, and professional services (maintenance, advisory services, etc.) being imported.

230. The government is aware of these weaknesses and of the industrial potential of the sector. In the short term, it plans to engage in support activities, supplementing the private sector in order to improve the rate of investment in the industrial sector and strengthen the growth and competitiveness of manufacturing industry in order to: (i) diversify industrial production; (ii) increase manufacturing value-added; and (iii) increase the share of manufacturing exports, including nontraditional exports, in the total. 
231. These actions include physical infrastructure development, institution building, and the implementation of policies supporting industry. First, the focus will be on providing selective direct support to competitive subsectors with high growth potential, taking into account opportunities in the national, regional, and global markets. Preliminary findings in early studies on the industrial subsector indicate that Cameroon could have a comparative advantage in agroindustry, textiles, and wood processing (see box below and Annex 3.3). Other activities include the strengthening of cross-sector services essential to industrial development, or sectors that could have a stimulus effect on industry. These sectors involve the production of key inputs for industrial subsectors or sectors that have a positive impact on the demand for industrial products. These are primarily sectors such as power (electricity and petroleum), transportation, financial intermediation, information and communications technologies, tourism, education, vocational and professional training, housing, and construction and public works.

232. The government is particularly committed to investing in the large-scale infrastructure needed for industrial development, including: (i) rehabilitation of the Wouri bridge, rehabilitation of the Douala and Yaoundé urban infrastructure, and construction of a bridge on the Mayo-Tsanaga; (ii) building infrastructure links between Cameroon, Gabon, Equatorial Guinea, the Central African Republic, Chad, and Nigeria; (iii) building a second bridge over the Wouri; and (iv) constructing a beltway highway around Douala with a connecting link to Limbe, where the harbor is expected to be developed as part of the ongoing redeployment of Cameroon's industrial shipyard.

233. Cameroon's industrial competitiveness depends upon a physical infrastructure and an institutional framework that should provide specialized services in the areas of standardization and quality control, promote exports and investment, create linkages between research institutions, training institutions and productive enterprises, and foster information dissemination and cross-industry relations. Experience in East and South East Asian countries has stressed the importance of combining physical and institutional infrastructures to strengthen industrial capacities. The government can also be an important driving force when working with private support organizations to foster development. The overall objective is to create an enabling environment for direct foreign and domestic investment. A wellfunctioning infrastructure will help reduce transaction costs while having a positive impact on the competitiveness of industrial enterprises and private investments in Cameroon's manufacturing sector.

234. The government plans to develop an industrial redeployment strategy and will take the following actions:

- Carry out an in-depth diagnostic study of the industrial sector: analyze opportunities, strengths, and weaknesses in order to identify and select competitive, high-growth sectors or subsectors, such as agrobusinesses, wood, aviculture, etc., or sectors with strongly positive externalities, such as construction and public works, energy, tourism, information and communications technologies (ICT), etc. The selection criteria will include export potential, the capacity to add value in manufacturing, technological expertise, the availability of critical factors, etc.;

- Prepare selective strategies to strengthen the competitiveness of the subsectors chosen, including specific incentive measures; 
- Analyze export promotion activities for subsectors catering to international markets, modernize export-oriented business in these subsectors, develop partnerships through professional meetings and investment forums, and finally, promote trade;

- Formulate a strategy to strengthen infrastructures that support industry (power, transportation, ICT, etc.), including the preparation and implementation of a powerful master plan, monitor the implementation of the transport sector master plan, prepare and implement an ICT master plan, and introduce the New Information and Communication Technologies Agency (ANTIC);

- $\quad$ Draft policy measures to promote local industrial outsourcing, including such activities as the creation of an outsourcing exchange, the development of industrial partnership arrangements through company visits, sector-focused professional meetings, bilateral partnerships, and the dissemination of information on business opportunities and public procurement;

- Conduct a diagnostic study of the industrial sector, support intermediary organizations, and devise specific strategies to develop their capacity;

- Study the creation of business clusters, consortiums, or networks to improve access to markets, input factors (raw materials, intermediate inputs, technology, etc.), and support services (training, marketing, technology, business opportunities, etc.);

- Evaluate the impact of privatization on the industrial sector by promoting and developing cross-industry relationships, outsourcing to local SMEs/SMIs, etc.;

- Improve research and development activities by creating a consultation framework for research institutes (such as IRAD and CIRAD), universities and other highereducation professional institutions, and businesses;

- $\quad$ Conduct out a human resource development study for the industrial sector.

235. The preliminary conclusions of the industrial subsector study conducted as part of the sources of growth study stress the importance of cluster development in the textile/garment industry, aviculture industries, tertiary wood processing sector, and tropical fruit processing sector. Annex 3.3 contains an analysis of these subsectors. 


\section{Box 15. The Comparative Advantage of Industrial Subsectors}

\section{Issues}

Because of the decline of the oil sector, growth recovery and poverty reduction will require the redeployment of the non-oil industry and high value-added services. Much as in the case of agriculture, the main issue is to identify subsectors with growth potential and in which Cameroon has a comparative advantage. This relates to the broader question of the competitiveness of Cameroon's economy and each of its sectors.

\section{a Methodology}

As for the agricultural subsectors, the competitiveness of these industrial subsectors is determined by the Domestic Resource Cost coefficient (DRC). Subsector competitiveness has been rated according to their DRC. The DRC measures, in terms of real resources, the opportunity cost of producing or saving foreign exchange. It is calculated by dividing the economic value of domestic resource inputs by the international economic value-added. (i) Values of DRC below 1 indicate an efficient use of resources. At this level, the cost of the foreign exchange earned or saved through domestic production is less than the international cost. (ii) Keeping in mind the effect of dynamic links and the fact that some subsectors that are noncompetitive in the short term could become competitive in the medium term with appropriate cross-sector policies, a second group of potentially competitive subsectors has been identified. These subsectors have DRC values ranging from 1 to 1.5. (iii) Subsectors with a DRC value of over 1.5 are considered noncompetitive. Negative DRCs indicate a very inefficient use of resources, corresponding to situations where free trade value-added is negative.

\section{autcome and lessons for a strategy}

The identification of competitive manufacturing subsectors in Cameroon is based on a sample of 44 companies, contributing about 85 percent of the non-oil industrial value-added. (i) Using the above classification, the most promising growth sector (group 1) includes the wood, wood processing, and beverage industries. The wood and wood processing industries merit the authorities' attention in the context of an industrial policy. (ii) Improving the competitiveness of agribusiness, chemical industries, and the rubber, textile, and steel industries should help boost industrial exports. These are potentially competitive subsectors in the medium term. Combined, groups 1 and 2 contribute about three-quarters of the value-added (76 percent) and account for over half of all manufacturing employment (58 percent). (iii) Finally, the cigarette and other manufacturing industries appear very costly and without a promising future. These results give selected and well-targeted support for an industrial redeployment strategy.

Cameroon - Competitiveness of Industrial Subsectors, 1997-2000

\begin{tabular}{|c|c|c|c|c|c|}
\hline Sectors & & $\begin{array}{c}\text { Average DRC } \\
1997-2000\end{array}$ & $\begin{array}{c}\% \text { industrial } \\
\text { value-added, } \\
1994-2000 \\
\end{array}$ & $\begin{array}{l}\% \text { industrial } \\
\text { employment in } \\
2000\end{array}$ & $\begin{array}{l}\text { Net exports (in millions } \\
\text { of CFA francs in } 2000\end{array}$ \\
\hline \multicolumn{6}{|c|}{ 1. Competitive subsectors } \\
\hline • & Wood & 0.97 & 11.66 & 8.79 & 60,117 \\
\hline - & Beverages & 0.88 & 16.49 & 3.41 & $-42,251$ \\
\hline \multicolumn{6}{|c|}{ 2. Marginally competitive subsectors } \\
\hline & Textiles & 1.30 & 10.13 & 5.25 & 53,396 \\
\hline - & Food products & 1.45 & 2.94 & 0.66 & 4,283 \\
\hline & Rubber & 1.28 & 12.05 & 35.89 & 49,171 \\
\hline & Chemicals & 1.47 & 4.42 & 1.78 & $-12,635$ \\
\hline • & Building materials & 1.41 & 6.06 & 0.82 & $-10,469$ \\
\hline - & Steel industry & 1.16 & 12.73 & 1.75 & 68,680 \\
\hline \multicolumn{6}{|c|}{ 3. Costly subsectors } \\
\hline$\bullet$ & Cigarette manufacturing & 2.8 & 2.33 & 0.73 & 1,072 \\
\hline$\bullet$ & Other manufacturing industries & 1.56 & 1.45 & 0.52 & 1,723 \\
\hline \multicolumn{6}{|c|}{ 4. Very costly subsectors } \\
\hline • & Mechanical appliances & * & 1.37 & 0.55 & $-3,581$ \\
\hline
\end{tabular}

* = Negative free trade value-added.

Source: Study on subsector competitiveness, Arsène Nkama, 2000. 


\subsubsection{Developing production support services with high value-added}

236. Similar to the manufacturing sector, with economic diversification there is room for considerable growth in the services industry. With a few exceptions, development in services is driven directly either by government (administrative, social, and infrastructure services) or by the productive sectors (trading services, such as commerce and finance). However, some services, such as tourism, depend on the natural endowment and on targeted policies.

\subsubsection{Tourism, cultural goods and services}

237. A list of all tourist sites prepared by the government reveals Cameroon's vast potential for various kinds of tourism (safari, ecological, seaside, cultural, etc.) in every region. More importantly, the study shows that Cameroon could exceed the current number of 250,000 tourists per year.

238. The government intends to develop the tourism industry by carrying out specific measures and actions, especially implementing a marketing plan prepared by the Ministry of Tourism. The sector development strategy currently being finalized therefore focuses on: (i) tourism promotion; (ii) the development of priority tourist sites (site selection, improved access, and development); (iii) the preparation of a tourist investment code; (iv) enhancing the statistical data collection system for the industry; (v) modernizing the Ngaoundéré National Hotel and Tourism School (ENAHT); (vi) conducting studies to prepare for integrating young graduates into tourism; and (vii) promoting ecotourism by creating a legal framework and test sites.

239. The government expects these actions to: (i) increase the number of domestic and foreign tourists; (ii) boost private investment in tourism-related subsectors, such as the hotel industry, food industry, handicrafts, and transportation; and (iii) create jobs and income opportunities as well as bring in foreign exchange.

240. The government also intends to develop a National Heritage Development Plan that reaffirms Cameroon's national identity, supports the development of tourism, and generates employment. Accordingly, it intends to carry out the following:

- Initiate a national heritage survey that would list, study, and publicize artistic, historical, archeological, or ethnological heritage resources;

- Facilitate the creation, production, and dissemination of works by Cameroonian authors, increase the supply of and demand for cultural goods, and enhance the social status of artists and authors;

- Create a National Museum of Cameroon, which would become the cornerstone of the government's policy for protecting and developing the natural heritage;

- Define and implement film and audiovisual production development strategies aimed at resuming film production and at creating and developing film-related professions;

- $\quad$ Create a National Art And Culture Institute that would serve as a development center for young artists, a life-long training center for all artists, and a center for training trainers in the different forms of art; and

- Improve access to books by opening a modern national library and public reading center. 


\subsubsection{Information and communications technologies (ICT)}

241. Following its liberalization in 1999 with the granting of mobile telephone licenses to two private operators (Orange and MTN), and although landline telephones continue to be managed by the traditional public utility (Cameroon telecommunications (CAMTEL)), growth in the telecommunications sector has been very strong. The number of fixed and mobile telephone subscribers rose from about 100,000 to 750,000 during 1999-2001, a 655 percent increase in three years. The subsector still has enormous growth potential, as the number current subscribers does not exceed 5 percent of Cameroon's total population and the current range of telecommunications products remains limited.

242. During the participatory consultation, the difficulty of accessing, and the lack of, information were mentioned as a determinant of poverty. Moreover, the United Nations considers access to information, this is, access to the knowledge required for basic life functions, to be a major human development indicator. Given the requirements of the New Economy, which is heavily reliant on information, communications, and artificial intelligence, the government will take measures to greatly improve citizens' access to information.

243. The government intends to: (i) open multimedia community centers in each of the 10 provinces to provide the landlocked population with Internet access to important health, education, agricultural, livestock, and environmental information; and (ii) install, with support from the UNDP and UNESCO, new rural radio stations in addition to the 15 already operational stations. The authorities also intend, through the Ministry of Communication, to support the national HIV/AIDS strategy through the implementation of a sector communication plan.

244. The government understands the economic potential of information and communications technologies (ICT) and is committed to promoting the development of this sector. In addition to reducing or eliminating some import duties and taxes for computer equipment, it created a National Information and Communication Technologies Agency (ANTIC) in April 2002. Within its missions, the agency must promote broader access to ICT as well as activities related to these new technologies, which are becoming increasingly popular among the population. 


\section{Box 16. The Community Telecenters Project}

The government has decided to equip 92 communities in rural areas with community telecenters, to promote integrated development in rural areas and improve the rural population's access to information resources via technological innovation. These are common infrastructures providing information and communications technology services to improve the population's standard of living, create income and employment, and to prevent rural outmigration.

This new type of partnership with the local government is part of an overall framework for universal access, connectivity, and reducing the digital gap between rural and urban areas. It also meets community aspirations expressed during the participatory process. The partnership will help: (i) create indirect employment by fostering the development of other activities around the telecenters, generating additional income for local governments; (ii) provide logistic support to the National Early Warning System of the Ministry of Agriculture; (iii) improve the population's standard of living by making farming more profitable and making price, quantity, and product market information available to producers; (iv) disseminate technical information to professionals in rural areas (doctors, teachers, agricultural producers, engineers, etc.); and (v) extend the telecommunication network's coverage to the more remote population.

Site selection for the community telecenters took into account the existence of commercial activities (border markets, cattle markets, cereal markets, major roads and border crossings, etc.), as well as synergy with other ongoing projects such as (i) the Belgium/Cameroon Telephone Extension Project connecting 67 localities and (ii) a project sponsored by the Japanese company NEC, connecting 21 localities.

The CFAF 1,445,000,000 Community Telecenter Project meets HIPC financial criteria. A pilot phase would create 12 test centers to: (i) verify the viability and feasibility of the project; (ii) evaluate the actual cost of installing telecenters; (iii) analyze results and assess the economic impact of telecenters on the environment; and (iv) foster the interest of other populations in telecenters and better target the population's needs.

Source: MINPOSTEL.

245. The Community Telecenter Development Project is an important component of the government's program (see box above). In addition to its direct benefits for communities, its development would help the government focus on improving information exchange between government agencies, perhaps developing a common format for the exchange of documents and information between different parts of the administration, thereby reducing the number of communication media linking public information systems.

246. In addition, the government's program plans to strengthen school and university structures, promote the development of computer centers, help deploy new technologies, and create techno-poles where businesses, public and/or private, would partner with universities to promote knowledge and know-how (for instance helping the development of distance learning capacities), improve school and university curriculums, and henceforth develop a national knowledge base needed to accelerate growth.

247. Specific measures will be taken to: (i) streamline secondary, technical, and professional education subsectors and programs; and (ii) provide schools with computer equipment and maintenance. At the same time, the government wants to use the fiber optic backbone being built with the Doba-Kribi pipeline to study the feasibility of extending this multifilament backbone to Douala and Bafoussam to create a high-speed triangular network that will considerably reduce transaction costs for businesses and communication costs for households. 


\subsubsection{Trade}

248. Domestic trade. More than a third of domestic trade is informal, making it difficult to develop owing to weak and obsolete means of transportation, a lack of professionalism in the business community, and poor distribution and payment systems.

249. The government intends to prepare and implement, in cooperation with the private sector and civil society, an external trade development strategy that will focus on: (i) better controlling marketing systems and ensuring oversight of and professionalism in trade activities; (ii) putting into effect trade facilitation sector policies (reducing official roadblocks, establishing standards, streamlining procedures, etc.); (iii) improving consumer protection; and (iv) preventing product adulteration and contraband.

250. Regional and international trade. The government's strategy was prepared in the context of the CAEMC customs union and Cameroon's membership in the World Trade Organization (WTO) agreements. Important progress is ongoing regarding the CAEMC: (i) the reduction of the common external tariff (to a maximum of 20 percent) as well as the number of tariff categories (from 5 to 4); and (ii) effective execution of the investment community charter provisions, specifically the provisions relating to trade and to the free movement of people, goods, and services.

251. Cameroon has also cosigned the WTO agreement to dismantle tariff barriers, in accordance with subregional agreements. The government will pursue ongoing trade and structural policies aimed at: (i) promoting an open trade and investment-friendly environment; (ii) protecting intellectual rights; and (iii) protecting the environment. In partnership with the private sector, the government intends to prepare an action plan that will help Cameroon's businesses take advantage of export opportunities (in particular in the textile sector) made available by provisions in the June 2000 Cotonou Agreement and the African Growth and Opportunity Act (AGOA) of the United States. 


\begin{abstract}
Box 17. A Single Processing Window for External Trade
Outcome after three years of operation. Cameroon's single processing window for external trade has become a successful example of public-private partnership for the entire subregion. Streamlining external trade procedures has facilitated trade throughout the subregion:

- Conditions are met for all goods to clear the harbor in less than 7 days when documentation is appropriate and is being processed and monitored by well-organized, professional services;

- Obtaining release warrants takes an average of 3.5 days compared to 12 days just three years ago; second vehicles are cleared in less than 24 hours, compared to 3 weeks before the installation of the one-stop office;

- Improved transit organization and performance, especially on the part of customs, where staffing has been increased accordingly.
\end{abstract}

Prospects. The first phase in establishing a physical one-stop office was successfully implemented. In the second phase, the challenge is to computerize the one-stop office to reduce processing time in a sustainable fashion and improve service quality in the Douala port. This would have several advantages:

Operational advantages: The one-stop office computerization will improve the speed and productivity of the business and administration organization. Transport businesses and administrations will have access to the most modern secure communication systems and will be in contact with the world at a minimal cost.

Economic and social advantages: The computerized one-stop office will facilitate trade (by procedures that are simplified, less burdensome, accelerated, and more reliable), strengthen competitiveness (through cost reduction and productivity gains), and improve customs revenues (more transparency and traffic increase). Studies have been conducted by the one-stop office and other port stakeholders. They found that in respect to external trade, transport operators could directly save between CFAF 12,000 and CFAF 15,000 per customs declaration, thereby creating incentives for shipping agencies to use the Douala port for subregional destinations. The reform will be ITC-based, and will improve professional skills and working conditions.

Competitive and strategic advantages: Competition between ports has become more intense, particularly in Africa. In addition to improved services, such as processing time, security, reliability, and the availability of new services to port users (electronic portal, electronic status information: physical, customs, administrative, goods), the urban infrastructure in Douala will also be modernized. These combined actions will strengthen the competitiveness and attractiveness of the autonomous port of Douala, making it a choice harbor for neighboring landlocked countries.

Source: External trade one-stop office.

\title{
3.3.3.4 Transport
}

252. The transport sector's growth has been strong (25.3 percent in 2001/02) owing to favorable economic conditions, such as projects like the Chad/Cameroon pipeline construction. Although the construction phase is coming to an end, Chad's economic recovery, spurred by oil revenues, should maintain the economic health of Cameroon's transport sector inasmuch as Chad's imports are essentially transiting through Douala.

253. The sector is expected to experience considerable growth, partly because of the real sector's spillover effect (product distribution) but also because of a deeper regional integration, allowing Cameroon to maximize its assets as a transit point. For instance, the Douala port reform and related investments will result in sustained medium-term growth for port activities. Furthermore, the construction of major roads through CAEMC and NEPAD (the Cameroon-Nigeria connection) will increase road transport, providing an essential extension to port activities (see details on this major transportation infrastructure development project in section 3.5.1). 


\subsection{REVITALIZING THE PRIVATE SECTOR}

\subsubsection{Improving the private sector environment}

254. Recent structural reforms have improved the business environment with measures aimed at liberalizing the economy, accelerating the state's retrenchment from productive activities, and thereby encouraging private sector development. These measures include price liberalization, the elimination of credit access problems, the simplification of customs procedures and tariffs, restructuring the banking sector, and transportation sector privatization and reforms.

255. Despite the progress made in private sector development, recent studies have shown the persistence of several obstacles. Broadly speaking, these obstacles are linked to excessive transportation and telecommunication service costs, insufficient power supply, cumbersome administrative and judicial systems, and the absence of sustained dialogue between the public and private sectors. These studies also identified specific constraints affecting SMEs/SMIs, microenterprise, and handicraft development, particularly in terms of policies and the support framework, and access to financing. There is still no SME/SMI development policy in this area, which would help the government impart strategic focus to its support for SMEs/SMIs and improve the division of labor and cooperation among partners (state, private sector, support organization, civil society, etc.).

256. To address these deficiencies, the government's private sector revitalization strategy, which is aimed at significantly reducing poverty, plans to create an enabling environment to improve business competitiveness, mobilize domestic resources, and attract foreign private investment. The strategy targets major businesses, SMEs/SMIs, microenterprises, and private sector support institutions. It relies on the following components:

- $\quad$ Promoting private sector support policies, institutions, and infrastructure;

- $\quad$ Strengthening private sector involvement through capacity building;

- $\quad$ Promoting policies targeting SMEs/SMIs, microenterprise, and handicraft development;

- Further mobilizing financial resources toward SMEs/SMIs and microenterprises;

- Improving the impact of the privatization program on SME/SMI development; and

- $\quad$ Strengthening the legal and regulatory framework of OHADA.

257. Private sector support policies, institutions, and infrastructure promotion. Activities will focus on: (i) improving the physical environment of business by speeding up the development of the transportation, telecommunications, power supply and distribution infrastructures; (ii) improving the institutional and regulatory framework, making public service delivery to businesses more effective; (iii) strengthening the legal security of investments by improving the judiciary system and enforcing business laws, particularly the OHADA laws; (iv) strengthening the mechanisms for consultation and dialogue between public and private sector support intermediaries within a broader private sector interministerial committee; (v) developing private sector support organization capacities; and (vi) implementing instruments and structures planned in the Investment Charter, including the Competitiveness Regulatory Council, the Investment and Export Promotion Agency, the Industry and Commerce Observatory, etc. 
258. Promotion of a public-private partnership (PPP) for the development of infrastructure. Given the scope of the country's requirements as regards transportation, power, housing, education, health, and tourism infrastructures, as well as the high cost of investment in these areas, the government intends to promote appropriate public-private partnerships, such as operational leases, concessions, lease or management contracts, build, operate, and transfer (BOT) contracts, or build, own and operate (BOO) contracts to attract national or foreign private investors to executing and/or managing such investments.

259. The investments mentioned above usually entail projects, as well as nonrecourse or limited recourse financing, with a limited government commitment, especially as regards noncommercial risks (political, legal, administrative risks) which fall outside the control of the private partner. The government understands that the relevant legal and financial arrangements are complex and will be attractive to the extent that the country's investment environment is secure. Therefore, the government has launched a study of the legal and institutional framework with a view to significantly improving private sector participation in infrastructure financing in Cameroon. One illustration of this is that invitations to bid have been sent to international private investors for a BOT to construct a second bridge over the Wouri River in Douala.

260. Many advantages and opportunities could come from increased private sector participation in the financing of infrastructures, along with better risk sharing between the public and private sectors by: (i) broadening the region's access to nontraditional financing; (ii) minimizing infrastructure costs through competitive bidding; (iii) improving the country's visibility on international capital markets (bonds, shares, commercial lending); (iv) accelerating infrastructure development so as to make it possible to focus public resources more on critical areas, such as health, education, etc; (v) transferring knowledge via local experts' participation in various technical areas; and (vi) creating jobs and fostering the participation of local businesses in infrastructure construction.

261. Increased private sector participation in capacity development. The government intends to considerably increase private sector involvement in the preparation and implementation of a youth training program, effectively creating appropriate institutional mechanisms and incentives. It will: (i) create accelerated professional training institutes; (ii) develop applied research programs in targeted economic and trade areas; (iii) finance large-scale computer learning programs; and (iv) finance entrepreneurship development programs in higher and tertiary education. These activities are designed to correct deficiencies in human resources, particularly in the productive sector.

262. Targeted policies for the development of SMEs/SMIs, microenterprise, and handicrafts. The government will design policies and specific action plans for these business categories with a view to: (i) strengthening the capacities of high-growth potential and competitive SMEs/SMIs; (ii) fostering microenterprise and handicraft development by promoting entrepreneurship, particularly among women, as well as by imparting practical and professional knowledge through better information dissemination; (iii) promoting business cluster or network development programs targeting SMEs/SMIs and microenterprises; and (iv) accelerating the 10 -year job creation program. This program is designed to create, within five years, about 300,000 jobs through 15,000 to 20,000 micro, small and medium-size enterprises (MSMEs). It will be financed by the government and the international community, including UNIDO, the UNDP, and the ILO. 
263. Mobilizing financial resources for SME/SMI and microenterprise development. Based on in-depth studies, the government intends to create an advisory assistance fund to help SMEs/SMIs overcome obstacles. The fund will be financed by the national budget as well as donor resources. It aims to improve the quality of the credit applications prepared by SMEs/SMIs, build their management capacity, and improve their credit access and capacity to repay. Other measures to improve the channeling of financial resources to the private sector, particularly SMEs/SMIs, are described in section 3.3.5 on financial intermediation.

264. Improving the impact of the privatization program. Activities will be pursued to: (i) improve the impact on the competitiveness of other sectors, which would ensue from better performing privatized infrastructure services in the areas of power, transportation, and telecommunications, and particularly through outsourcing to SMEs/SMIs; (ii) make retraining or outplacement programs more efficient; and (iii) strengthen the competitive framework.

265. Legal and regulatory framework in the OHADA context. Priority will be given to strengthening the regulatory and legal framework, a prerequisite for future investments. Accordingly, the government will continue to implement new business legislation through the Organization for the Harmonization of Business Law in Africa (OHADA), of which Cameroon is a member. It will also fulfill its commitments outlined in the Investment Charter, particularly those relative to (i) protecting business and investment freedom; (ii) securing investments through the establishment of an institutional and regulatory framework; (iii) supporting investors and prompt dispute settlement procedures in respect of commercial and industrial investment and activities; and (iv) implementing an attractive and incentive taxation system for investors. In addition, promoting a competitive environment across all sectors is essential for private sector development, and especially for SMEs/SMIs.

\subsubsection{Financial intermediation in support of the private sector}

266. Cameroon's government has already carried out an in-depth restructuring of the national financial sector, enabling this vital sector of its economy to improve its contribution to financing growth and reducing poverty. Despite these efforts, the banking sector still lacks resources to finance medium- to long-term loans. Moreover, access to credit and production factors, a prerequisite for increased economic participation by the poor, remains very difficult.

267. Indeed, financial intermediation remains shallow, with a ratio of money supply to GDP at 19.5 percent in 2002 . The traditional banking sector has about 250,000 customers, while the decentralized financial sector has 200,000 customers, totaling 450,000 customers for a population of 15 million, reflecting the low rate of bank penetration. Similarly, the insurance sector has few products despite stronger monitoring of the sectors, which has improved its functioning. These statistics bear witness to the considerable potential for financial sector development in the years ahead. The government will continue to implement its financial intermediation diversification and strengthening policy.

268. The banking sector. The government has just completed a bank restructuring to improve their financial soundness. The Central Africa Banking Commission (COBAC) now supervises the banking system. To strengthen the reach of the sector, the government intends, through a reform of the postal financial system and a deeper restructuring of the banking sector, to promote the development of modern structures to mobilize savings and finance economic activities in secondary towns and rural areas. The government will also strictly enforce the commitment made by the party taking over the Banque internationale $d u$ 
Cameroun pour l'Epargne et le Crédit (BICEC), in the context of its privatization, to open new branches and offices close to economic operators, such as tradesmen, small retailers, SMEs/SMIs, and those operating in rural areas.

269. Nonbank financial sector. The government intends to strengthen the implementation of the Douala Stock Exchange (DSX) to effectively channel investors' financial resources to potential users. Accordingly, emphasis will be placed on: (i) DSX management; (ii) promoting, monitoring, and controlling exchange operations; (iii) promoting individual investments; and (iv) preparing and enforcing judicial, legal, and regulatory provisions to protect operators against various crimes, such as insider trading.

270. The government also intends over time, on the basis of in-depth studies, to restore specialized financial institutions while partnering with the private sector in such areas as external trade, agriculture, and SMEs/SMIs. In addition to these institutions, it will enhance financial intermediation through the diversification of financial instruments, such as leasing, risk capital, mutual guarantees, mutual fund investment arrangements (OPCVM, SICAV, and Collective Investment Fund), guarantee funds, or regional development funds to be financed through budget allocations and by external financing. Feasibility studies will be conducted prior to the creation of such funds.

271. In partnering with insurance operators, the government will undertake activities to (i) promote the need for medical insurance coverage, life insurance, and mutual provident schemes with the help of administrative, medical, and political authorities; (ii) improve the accountability and supervision of risk insurance for fire, transportation, building construction, etc.; and (iii) insure public real property or real estate assets.

272. Decentralized financial sector. Cameroon's decentralized financial sector has grown very quickly over the past two decades because of problems in the traditional sector and the expansion of informal economic activities. The combination of structural problems and banking sector crises has prompted strong expansion in microfinance saving and credit structures, including savings and credit cooperatives (COOPEC), the Cameroon Cooperative Credit Union League network (CAMCCUL), the Mutuelles Communautaires de Croissance (MC2) (a community level mutual fund network), the village level savings and loan association under the decentralized rural credit pilot project $(\mathrm{CV})$, and the Cameroon development ACEP project.

273. However, the sector has grown without an appropriate regulatory framework, such as monitoring and surveillance systems such as COBAC. This shortcoming resulted in a lack of protection for depositors and in operations that fail to meet prudential standards. This led the government to establish operating modalities for savings and loan cooperatives. In particular, the government has taken measures to protect investors. In addition to revising the 1992 law on cooperatives, it placed COOPEC under COBAC's control. The government will rely on a decentralized financial system and try to ensure that the poor have access to credit. It will enforce: (i) the COBAC treaty provisions for COOPEC; (ii) the appropriate supervision of existing cooperatives in accordance with the regulations in force; and (iii) appropriate execution of the national microfinance support program (PPMF). Furthermore, the government will continue restructuring the savings and loan sector to make savings more secure and to ensure that the resources mobilized become a strong catalyst for development. 
274. The government intends to strengthen the monitoring capacity in the national microfinance program (PPMF) in order to further mobilize individual investor savings in favor of microenterprises. The success of these initiatives will be measured by their capacity to: (i) increase the lending volume of the microfinance institutions (MFIs) at the local and sector level; (ii) promote good business relations between the MFIs and commercial banks in order to introduce microcredits to their portfolios; (iii) develop new institutions and/or new microfinance networks; (iv) improve small business performance as well as microfinance institutions; (v) help microenterprises evolve into small-scale enterprises; (vi) strengthen the support capacity of Cameroon's association of microcredit institutions; and (vii) develop nonfinancial support services for microenterprise (business development centers, workshops, handicraft houses).

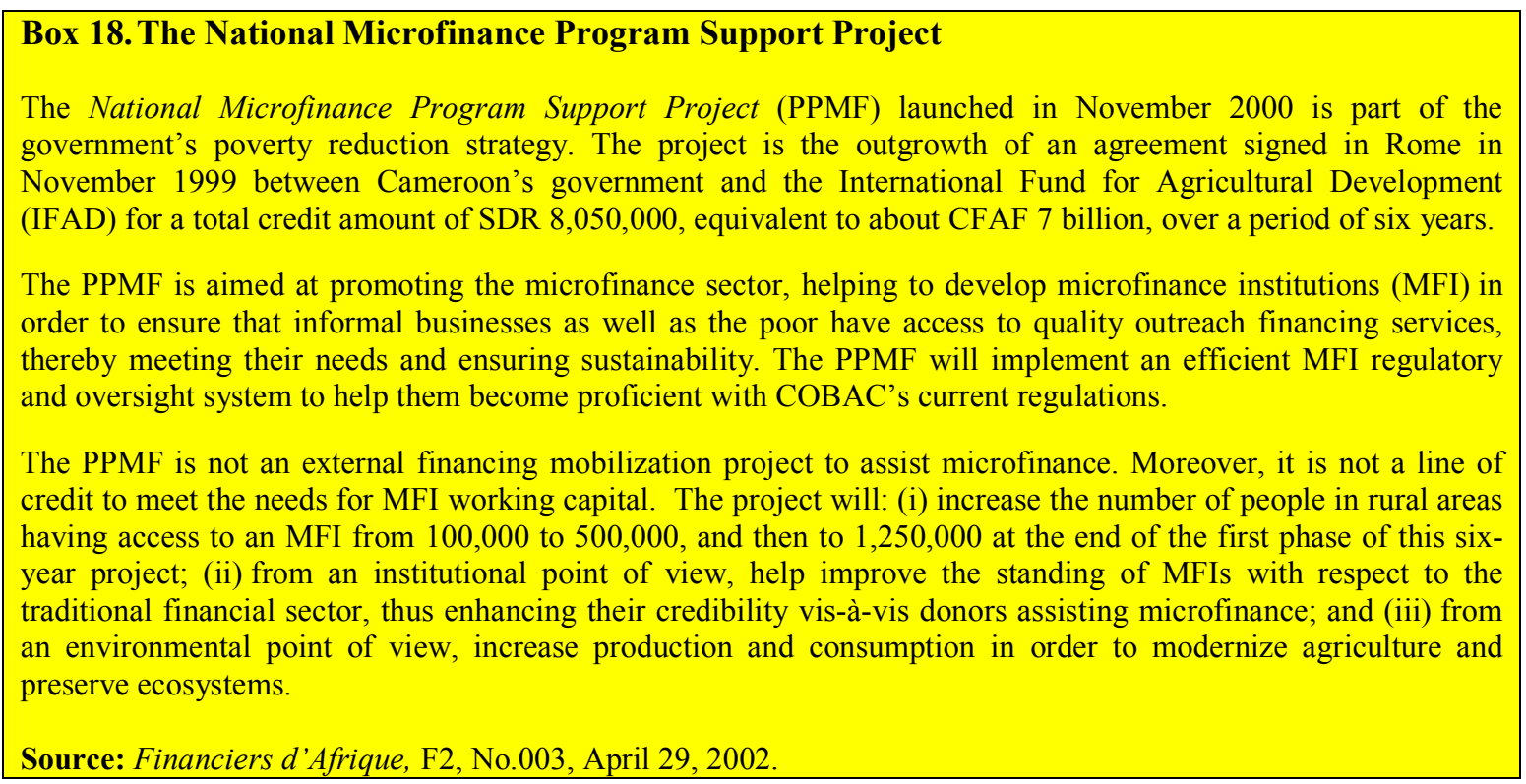

\subsection{INFRASTRUCTURE, NATURAL RESOURCES, AND ENVIRONMENT}

\subsubsection{Transportation infrastructure}

275. During the 1980s, the transportation infrastructure significantly deteriorated because of a lack of road maintenance, the absence of new road construction, inadequate dredging of the Douala port approach channel and other bodies of water, and the lack of railroad track maintenance. During the participatory consultation, the population stressed that improving infrastructure was a major priority.

276. Since 1996, the government has been executing the Transport Sector Program (PST) that it prepared with its partners. The program provides a comprehensive framework for investment to rehabilitate the country's infrastructure and has had some significant achievements: (i) creating a Road Fund (RF) in 1998 to secure resources for road maintenance; (ii) signing a concession contract for the railway; and (iii) liberalizing the maritime sector. The strong growth in the transportation sector is the consequence of favorable economic conditions, including the Chad-Cameroon pipeline project.

277. Additional ongoing reforms include: (i) in rural transport, the preparation and adoption of a rural road rehabilitation and maintenance strategy; (ii) preparation of additional strategies 
in river and lake transport, taking advantage of Cameroon launching its rural transport program (PTMR); and (iii) strategies aimed at developing transport means and services. The government has also carried out an important reform of the harbor sector.

\subsubsection{Road infrastructures}

278. Road and rural road infrastructure rehabilitation and development are priorities in the government's poverty reduction strategy. To ensure that the transport and public works sector fully contributes to Cameroon's development, the government has prepared, specifically for road infrastructure, a transport and public works sector strategy that has achieved the following objectives: (i) defining a priority road network allowing efficient allocation of available resources; (ii) refocusing the state's mission on planning, programming, and monitoring; (iii) privatizing road maintenance, outsourcing it to SMEs; and (iv) making more efficient use of road network maintenance and development financing.

\section{Box 19. The Transport and Public Works Sector Strategy}

The economic crisis that led to accelerated deterioration of the road network marked the start of a reform process, with the government's adoption in 1996 of a transport sector strategy aimed at allocating more funds to road maintenance and development. Regarding the road subsector, the strategy has achieved the following:

- $\quad$ Definition of a priority road network to which most available resources will be allocated;

- $\quad$ Refocusing of the state's mission on planning, programming, and monitoring;

- $\quad$ Privatizing road maintenance, now outsourced to SMEs/SMIs.

In Cameroon, the Transport Sector Program (PST) includes all sector-specific strategies and serves as the framework for all road investments. It is supported by traditional development partners as well as by the Road Management Initiative (RMI) and the Rural Transport Program (PTMR), two programs financed by external partners under World Bank leadership of the Sub-Saharan Transport Policy Program.

Significant results have been achieved, particularly with the 1998 implementation of a Road Fund securing road maintenance resources. This scheme has considerably shortened payment to private enterprises, improved the programming of road maintenance, and helped revitalize private civil engineering and construction firms.

Source: MINTP. 


\section{Box 20. Rural Road Maintenance and Rehabilitation Strategy}

As an integral component of the transport sector strategy, the Rural Road Maintenance and Rehabilitation Strategy adopted by the government is based on community participation and the devolution of rural road management to the communes. It is being implemented as an IDA-financed pilot project that tests the sharing of responsibilities for the rehabilitation and maintenance of the priority road network between the central and local governments in the Center, Northwest, and Southern provinces, following a media campaign to raise awareness in the public. The central government will finance the rehabilitation of the network while communes (the bottom echelon in local government) and the beneficiary population will take responsibility for routine road maintenance once the roads have been repaired. Other rural road programs financed by other donors (AFD, AfDB, IDB, and EU) are being implemented under the same strategy, with a goal of rehabilitating about 8,000 kilometers of rural roads within the next few years by the central government, with routine maintenance being provided locally.

In the context of the government's strategy to strengthen decentralized structures, the communes will assume full ownership of rural roads and become responsible for their maintenance once their rehabilitation has been financed by the central government. Maintenance at the commune level will include small- or medium-scale activities. For efficiency reasons, the communes will outsource maintenance to local SMEs, organizations, or existing associations.

The above provisions are part of a rural road management law project, which will translate into legal terms the rural road management and rehabilitation strategy adopted by the government and supported by all stakeholders, including the central administration, communes, civil society, and donors, following wide consultations at the central and provincial levels.

The law has the following objectives:

- Reducing poverty by improving accessibility and standards of living in rural areas;

- Bottom-up partnership development, resulting in greater participation of road site populations and road users in the management of rural roads, and particularly in financing, maintaining, and safeguarding them;

- Implementing a new institutional framework by: (i) refocusing Ministry of Public Works services on planning, programming, budget management, control and monitoring, and evaluation; (ii) devolving to the rural communes the responsibilities and competencies needed to create and maintain rural roads; (iii) creating rural road management coordination and consultation structures at the provincial level, with municipal councils; (iv) reinforcing the mayors' prerogative of the public power to prevent, and to institute legal proceedings and punish, any willful damages to the rural road network (following a simplified and accelerated procedure); and

- Organizing a sustainable rural road maintenance fund ensuring financial availability and quick and efficient transparent payment procedures.

Source: MINTP.

279. Priority programs. The public works and transport sector strategy is reflected in a number of priority programs in the PRSP. The government intends to (i) extend the transAfrican road in NEPAD; (ii) develop a subregional road network in the CAEMC zone; (iii) improve national network trunk roads; and (iv) improve maintenance of the existing network.

280. Program 1: The trans-African road. Cameroon represents a development pole for both subregions because of its strategic position between West and Central Africa. Implementing the former design of the trans-African road is therefore a development priority. The road links Nigeria to Central Africa, crossing Cameroon at Ekok, Mamgé, Bamenda, Bafoussam, Foumban, Nyamboya, Banyo, Tibati, Meidougou, and Garoua-Boulaï.

281. Program 2: CAEMC subregional network. The roads in this network link Cameroon to the neighboring CAEMC countries, including Equatorial Guinea, Gabon, Chad, the Central 
African Republic, and Congo. The main trunk roads are: (i) Ambam-Kyé-Ossi; (ii) AmbanEking; (iii) Ngaoundere-Touboro-Moundou (Chad); (iv) Garoua-Demsa-Nigeria border; (v) Mora-Limani-Banki (Nigeria); (vi) Sangmélima-Djoum-Congo border; and (vii) Kousséri- N'djaména (Chad).

282. Program 3: National network trunk roads. Good trunk roads are needed to meet the requirements of decentralization (linking the national capital to provincial capitals) and to permit the socioeconomic development of landlocked areas (linking the industrial harbor centers, consumption centers, and tourist sites). It is planned to extend the current paved road network to ensure connection between: (i) the two major urban centers in the country (Douala and Yaoundé) and/or provincial capitals; (ii) department capitals to provincial capitals; and (iii) major rural agricultural production areas to their natural markets, usually urban centers.

283. Program 4: Safeguarding the existing road network system. The existing road system should be maintained to ensure its sustainability and functionality. Therefore, rural roads have a high priority. In the New Rural Road Maintenance Strategy (NRRMW), road maintenance will be devolved to deconcentrated entities with a greater involvement of beneficiary populations. The strategy departs from the policies governing other road networks, in that it is more community-based and decentralized, and focuses on implementing labor-intensive activities. These also improve the accessibility of villages, plantations, and factories, facilitate the collection of agricultural products and the shipment thereof to markets and urban centers, and promote the supply of consumable and agricultural or industrial inputs to rural areas.

284. Auxiliary measures will be included in the rural road project, organizing the training of road site populations as well as road maintenance committees responsible for routine maintenance of the rehabilitated network, allowing effective control over the road network by local governments.

\subsubsection{River and lake transportation}

285. River and lake transportation has witnessed a strong development in areas where rivers and lakes are navigable. It is one of the most important means of transportation as it ensures the mobility of people in these areas and is virtually the only means of transportation for their freight. However, it is still penalized by poor lending, loading and unloading arrangements, and the lack of organized docking, resulting in the deterioration and loss of goods.

286. To correct these deficiencies, the government's strategy in this area will: (i) create a structure to define and support the river and lake transportation development policy; (ii) formulate, in consultation with the private sector, a well-adapted legal and regulatory framework to improve the management and organization of such transportation; (iii) launch a pilot project, in partnership with the private sector; (iv) plan a strategic investment program; and (v) put in place a monitoring and evaluation scheme to better focus, organize, stimulate, and manage the supply of and demand for river and lake transport. Similarly, the government will finalize a strategy to promote less costly intermediate community transportation.

\subsubsection{Port activities}

287. The Douala harbor has a capacity of 7.5 million metric tons per year, which could be extended to 10 million. The volume of traffic was about 6.3 million metric tons in 2002 , with an average growth rate of 8 percent per annum since 1994. The port has a strategic position at 
the bottom of the Gulf of Guinea that allows it to play an essential role in all Central African countries.

288. A large port reform program is being implemented to strengthen port performance. The key features of the reform include major institutional restructuring and infrastructure modernization and rehabilitation activities, as well as the transfer of management responsibilities for part of the industrial and commercial sectors to the private sector. These activities include the operation, management, and maintenance of the modernized container terminal in the port of Douala as well as towage and marine services in the port. The reform has been complemented by the establishment of a single processing window for external trade (GUCE) to reduce product handling and transit costs.

\subsubsection{Construction and public works}

289. The government will take appropriate measures to promote this branch of industry to substantially reduce construction costs in Cameroon. Emphasis will be placed on (i) improving the subsector's efficiency (raw material processing, manufacturing and import of cement products and structural iron, etc.); (ii) enhancing the professionalism of SMEs/SMIs engaged in construction and promoting subcontracting to local SMEs/SMIs through international consortiums and other protective measures; (iii) promoting quality assurance in construction; (iv) initiating nationwide programs (social housing, roads, harbors, airports, etc.) to step up the contribution of construction and public works to GDP growth; (v) developing the subsector labor pool; and (vi) promoting new ways to finance infrastructure, including management or concession contracts relating to public/private partnerships developed for major public works.

\subsubsection{Natural resources and environment}

290. As Cameroon is richly endowed with natural resources, it has the potential to become self-sufficient in energy. This potential should be developed to stimulate economic development and reduce poverty.

\subsubsection{Mining and power}

291. In the mining sector, several deposits have been identified. Despite the promulgation of the mining code and its implementing provisions, investment continues to be scarce. The government will consequently make the new legislative and regulatory framework more attractive for large-scale investments in the sector. To this end, the authorities will finalize studies to make available specific information on the real potential of existing deposits and on prospecting for new deposits.

292. Promulgation of the mining and gas codes by the government clearly demonstrates its willingness to develop both sectors. In the area of solid mining, the new code guarantees the operator investment safety and provides incentives for the exploration and development phases. All those measures are designed to increase the chances of developing Cameroon's mining resources.

293. In the hydrocarbons sector, proven reserves and oil production are steadily decreasing. However, as part of the effort to implement a new oil code, the government will pursue oil prospecting on the mainland and in the open sea. In the oil product subsector, the regulatory framework will also introduce competition in the downstream activities of the oil sector, which will result in job creation in the medium and long term through the promotion of local 
small- and medium-sized enterprises and industries (SMEs/SMIs). For efficiency purposes, the government will adopt a package of measures seeking to: (i) elucidate the role of the various operators involved in the hydrocarbons sector; and (ii) promote prospecting and private investment in the sector.

294. As regards the electricity subsector, despite the privatization of the power utility, Société Nationale d'Electricité (SONEL) and the existence of a legal and regulatory framework liberalizing and introducing competition in the electricity subsector, the supply of electrical energy and the quality of service rendered to the public in general still have huge shortcomings. In the face of such difficulties, the government has opted for a strategy aimed at: (i) facilitating maximum development of the existing potential, through appropriate incentives giving priority to basic hydroelectricity, additional natural gas, and renewable energy for remote areas not yet connected to the interconnected grid; and (ii) making sector management more transparent and efficient.

295. A new institutional framework was established with the creation of the Agence de Régulation du Secteur de l'Electricité (ARSEL) and the Agence d'Electrification Rurale (AER). The new framework opens the way for competition in the electricity sector. The specifications for the agreement governing the concession of SONEL provide for substantial investment to meet the high demand for electrical energy at a time when large-scale industrial investment projects are under consideration. At that level, the contribution of the government in its capacity as grantor is essential for the success of the investments.

296. On the basis of the different options open to the nation, the government will look into the best methods of supplying energy to the productive sector as well as to households. The authorities therefore plan, in the medium and long term, to build hydroelectric power plants, thermal stations, and ministations or other plants in areas outside the interconnected grid.

\subsubsection{Water and sanitation}

297. Problems of village water engineering and access to safe drinking water appeared to be a major handicap to the grassroots populations, according to the findings of the participatory consultations. Besides the main urban centers where coverage in safe drinking water is still to be improved, the rural world is still confronted with an acute water problem because of the adverse geoecological situation of certain regions and lack of an appropriate policy for the sector. Access to safe drinking water is low considering the average per capita income in Cameroon. It was estimated at 86.2 percent in urban areas and 31.3 percent for rural areas in 2001 .

298. Faced with that situation with multifaceted repercussions (impact on public health, output, and agropastoral methods and techniques), the government has undertaken to implement a series of reforms, including the privatization of the Water Corporation (SNEC), which constitutes one of the major components. Such reforms are designed to promote access for all to safe drinking water by 2025 by substantially reinforcing the water supply projects approved under the "Rural Water Supply II" Program.

299. That program seeks to: (i) substantially improve the current coverage rate of the rural areas in terms of safe drinking water and raise the access rate to 75 percent by 2015 ; (ii) make adequate sanitation services available for the protection and evaluation of the quality and quantity of water, taking into account the protection of the natural ecosystem, public health, 
and human resource development, and (iii) to identify objective and relevant indicators for the programming and integrated management of safe drinking water and sanitation projects.

300. Besides extension and rehabilitation of the safe drinking water supply plants and pipeborne water programs, drilling of boreholes and wells will, as a matter of priority, be accelerated in underprivileged areas. The specific medium-term objective is to implement a program for the production and distribution of safe drinking water in 113 secondary and densely populated semi-urban centers.

301. On the basis of available studies as well as hydrogeological considerations and the various ongoing programs jointly implemented with various development partners, a comprehensive program for the construction of boreholes and mini water supply systems was launched with HIPC funding. In FY03, considered a pilot period, it is also envisaged to acquire mobile water stations capable of supplying safe drinking during emergencies (severe water shortages in built-up areas and health facilities, severe drought, epidemics, civil and humanitarian incidents, volcanic eruptions, floods). Simultaneously, a safe drinking water supply program is envisaged for schools and health centers throughout the national territory. That program will be backed by a package of sanitation measures principally in urban and semi-urban areas, focusing on wastewater treatment, extension of the primary sanitation network, and the construction of new water purification plants.

\subsubsection{Environment}

302. Cameroon enjoys a striking ecological, cultural, and anthropological diversity. Nearly 90 percent of African ecosystems are represented there and are divided into vast Sahelian, Sudanese, forest, mountain, marine, and coastal zones. Development of those ecosystems has always been conducted in a disorganized manner, thus resulting in the destruction of vast ecosystems in the last few decades. Such damage primarily derives from diverse phenomena provoked and sustained by conscious or unconscious action by man-poaching, overgrazing, uncontrolled bush fires, and shifting agriculture.

303. Cameroon has among the richest, most diverse wildlife on the African continent, including about 409 species of mammals, 183 species of reptiles, 849 species of birds, 190 species of amphibians, and 39 species of butterflies. In order to preserve certain ecosystems, Cameroon opted for management of biodiversity through a network of protected areas. All the wildlife reserves in 2002 covered about 14 percent of the national territory, or $6,656,000$ hectares. Among other facilities, the network includes ten national parks, six wildlife reserves, one wildlife sanctuary, three zoological gardens, 35 hunting zones, ten hunting zones under community management, etc.

304. In Cameroon, forests covering over 22 million hectares have been exploited for several decades now for lumber, timber, and firewood. Other nonligneous resources such as its wildlife and other forest products have been as well. That kind of exploitation does not go without perceptible impact on the environment. As a matter of fact, the forest is receding by about 100,000 hectares per annum.

305. In respect of biodiversity, in the last several decades Cameroon has recorded significant damage to its ecosystems (marine and coastal, humid tropical forest, and wooded tropical savannah notably) following the unsustainable exploitation of its biological resources. Such exploitation is due among other things to: (i) destructive agricultural, forestry, and pastoral 
practices; (ii) population pressure; and (iii) overexploitation of forest, water, wildlife, and floral resources.

306. Despite the existence of a Framework Law on environmental management and the adoption of the poaching control strategy included as part of the Emergency Plan of Action, coupled with the establishment of an Interministerial Environment Committee, poaching and biodiversity degradation still remain acute. To remedy the situation and preserve the various ecosystems, the government formulated a coherent strategy embodying the principles adopted in AGENDA 21 in Rio in 1992.

307. The strategy places emphasis on environmental appraisals, notably the impact studies required for any project pertaining to development, engineering works, equipment, or installation liable to damage the environment, as well as on environmental audits. Furthermore, the government has formulated a biodiversity strategy and an associated action plan. Implementation of that action plan will help promote sustainable management and exploitation of the various ecosystems and thereby (i) comply with international conventions, regional agreements, and sector plans on biodiversity, such as the International Convention on Intervention in the High Seas, the Kano Convention, the National Forestry Plan; and (ii) draw up a contingency plan for the management of hydrometeorological and/or geophysical disasters, etc.

\subsection{ACCELERATING REGIONAL INTEGRATION WITHIN CAEMC}

308. Cameroon constitutes a pole of growth within CAEMC. The country will pursue an openness and cooperation strategy within CAEMC in order to broaden the scope of markets while ensuring the overall competitiveness of the zone in relation to the rest of the world. The openness policy may induce a host of multiplier effects and, depending on the nature of their impact on the various countries, increase the stability and intensity of growth. Cameroon undertakes to maintain fiscal discipline as required by the convergence criteria, to enhance the depth of its financial and interbank markets, and to improve the physical infrastructure to allow for better integration of the labor, goods, and capital markets. Cameroon intends to rely on NEPAD in order to revitalize the regional integration process.

\subsubsection{Macroeconomic Policies}

309. Monetary policy. The government of Cameroon has undertaken to pursue efforts toward the modernization of the monetary policy within CAEMC, to achieve greater market liberalization. That policy must be reflected in (i) the progressive elimination of direct advances from the Central Bank to states with effect from 2003 (by one-tenth per annum over the next 10 years); and (ii) the creation of a regional state securities and private bond market. The government is determined to effectively implement the new exchange regulations in the CAEMC zone, particularly the devolution of authority for the management of foreign transactions from the public administrations to the commercial banks.

310. Fiscal Policy. To encourage the harmonization of development policies within the CAEMC zone, the member states have adopted macroeconomic convergence criteria. In keeping with these criteria: (i) the budget balance of each member state must be positive or nil by 2004; (ii) the overall public debt of each member country must not exceed 70 percent of GDP by 2004; (iii) domestic and external payments arrears must not be allowed to accumulate and must be eliminated by 2004 ; and (iv) the annual inflation rate in each country must not 
exceed 3 percent. Efforts are underway to secure tax harmonization in the zone, notably by introducing and harmonizing the value-added tax (VAT).

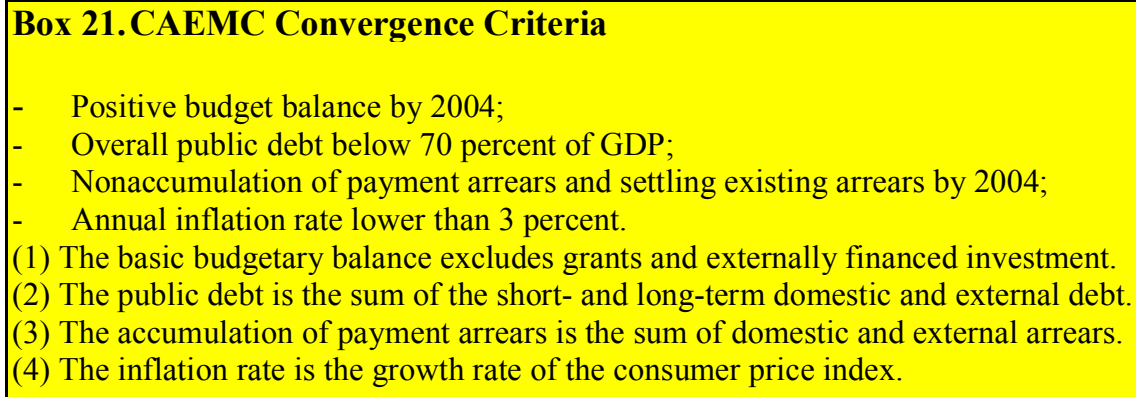

\subsubsection{Trade policies}

311. Trade policy and the Customs Union: In this regard, Cameroon will play a key role in defining an action plan to eliminate the shortcomings observed during implementation of the 1994 tax and customs reform (complexity of customs procedures, weaknesses in the transit system, etc.) and the informal border constraints (presence of numerous unofficial roadblocks). The government will review nontariff barriers in order to encourage a second reform aimed at streamlining and further liberalizing trade systems and introducing possible harmonization with neighboring regional groupings. In particular, Cameroon is committed to: (i) playing a major role in reinforcing regional integration within the BEAC zone through a substantial reduction in the common external tariff (to a maximum of 20 percent) and in the number of categories (from 5 to 4); (ii) specifying the practical and operational modalities of the common Investment Charter within CAEMC; and (iii) accelerating the establishment of the Community Development Fund (FODEC), as well as fostering cooperation with other Member States.

\subsubsection{Financial policy}

312. The government has just completed the reorganization of the system. Single-tier approval will henceforth help banks open branches in any member country. The government will pursue efforts to improve financial deepening through: (i) the implementation of reforms in the payment system (supported by IDA); and (ii) application of the regional legislation on microfinance adopted by COBAC. Furthermore, the government intends to speed up the operational phase of the Douala Securities Exchange. Efforts are underway to set up a common framework.

\subsubsection{Infrastructure}

313. Road transport: The CAEMC has drawn up a transportation master plan targeting the construction and rehabilitation of over 10,000 kilometers of roads by 2006 to link four of five capital cities within the zone. Links with Nigeria and the Democratic Republic of Congo (2,000 kilometers) will be established after 2006. Several donors (EU, ADB, World Bank, AFD, etc.) which have been involved in funding the road network for a number of years would support the extension projects.

314. In more concrete terms, the objective is the construction of asphalted roads in the medium term to facilitate traffic flow from one country to another. Cases in point are (i) the 
Cameroon-Central African Republic highway, with the construction of the Bertoua-GarouaBoulaï road covering a distance of 247 kilometers, which has been operational for over a year; (ii) the Cameroon-Chad highway, with the construction of the Ngaoundere (Cameroon)Moundou (Chad) road covering a distance of 393 kilometers, including 265 kilometers within Cameroonian territory. This road functions on the principle of rail and road complementarities; (iii) the regional Gabon-Cameroon and Cameroon-Equatorial Guinea highways, with the proposed construction of two bridges, one spanning the Ntem River at Eboro, along the Yaoundé-Libreville highway; the other spanning the Ngoazik River along the Cameroon-Equatorial Guinea highway. The recent inauguration of the Nsimalen-Ebolowa highway in 2002 constitutes a decisive step toward linking Yaoundé to border towns in Gabon and Equatorial Guinea by asphalted roads.

315. Maritime/River transportation. Cameroon is in the process of completing institutional port reforms and transferring industrial and commercial activities to the private sector. Reduced costs and shorter delays will help improve competitiveness, benefiting all the countries of the subregion, notably the Central African Republic and Chad.

316. Air transportation. The liberalization of air transportation will be progressively implemented in accordance with the framework established by the Yamoussoukro Decision (2000). In particular, Cameroon will abide by ICAO rules to improve air transportation safety. Furthermore, studies and consultations are underway for the creation of a subregional airline.

317. Telecommunications. In an effort to consolidate the achievements toward economic and social integration, CAEMC Member States decided to promote the interconnection of their telecommunications networks. The Terms of Reference for the feasibility studies on the interconnection of the Telecommunications Networks of CAEMC Member States were adopted in August 2002. A Community Telecommunications Code is under formulation.

\subsubsection{Labor market}

318. Convinced that subregional integration is an effective strategy for its insertion into an increasingly global economy, Cameroon and the other CAEMC Member States agreed to accelerate a number of actions, including the immediate introduction of the CAEMC passport and the elimination of all impediments to the free movement of persons, goods, services, and capital.

\subsubsection{Areas for sector cooperation}

319. Forestry. Cameroon will reinforce regional cooperation in the sustainable conservation and management of the forests in Central Africa through COFIMAC, which is responsible for ensuring the proper implementation of forestry policies, conservation, and the protection of protected trans-border zones.

320. Health. As part of HIV/AIDS control efforts, the government will focus among other things on the regional road and river transportation corridors (particularly the Congo-Ubangi river corridor and Lake Chad). The actions will be supported by the UNAIDS program and the AfDB.

321. Education. Cameroon undertakes to maintain its support for subregional training institutions, namely, the Ecole Inter-Etats des Douanes (EIED) (Customs); the Institut Sousrégional multisectoriel de technologie appliquée, de planification et d'évaluation de projets 
(ISTA) (economic planning); and the Institut Sous-régional de Statistique et d'Economie Appliquée (ISSEA) (statistics).

322. Tourism. A Permanent Tourism Commission was created within the CAEMC. This Commission is responsible for all matters pertaining to tourism and especially for formulating any measures to promote tourism in the CAEMC zone.

\subsection{STRENGTHENING HUMAN RESOURCES AND THE SOCIAL SECTOR}

323. The population of Cameroon is estimated at 15.5 million inhabitants in 2002, with an average age of about 22. It is thus an essentially young population which is growing at an average annual rate of 3 percent. At that rate, it is expected to reach about 24 million by 2015 . This rapid growth will require the accelerated creation of wealth in order to meet the basic needs of the population and facilitate access by all to essential basic social services.

324. Faced with this challenge, the government intends to promote the national population policy that was revised and adopted in March 2002. That policy seeks to achieve a suitable match between population growth, progressive human resource development, and the available accessible resources. The policy primarily seeks: (i) improvement in the health status of the population in general, and that of mothers and children in particular; (ii) the promotion of basic education for all, and especially for girls; (iii) intensification of the fight against unemployment; (iv) promotion of gender equality and equity; (v) environmental preservation; and (vi) improvement of the conditions necessary for safeguarding the wellbeing of the family and the individual, thereby ensuring personal advancement.

325. Beyond those social objectives, the human resource development strategy constitutes the lynchpin of the private sector growth and support strategy. It seeks to enhance Cameroon's human capital and thus improve the overall competitiveness of the economy. To that end, the government created a Ministerial Department in August 2002 and will step up investment in technical education and vocational training. The development of vocational skills will require improving technical and vocational training that is essentially oriented toward the aforementioned sectors to be developed. Institutional mechanisms will be introduced to encourage greater private sector involvement in the formulation and implementation of training programs, including specific measures for vocational capacity building. Those guidelines are reflected in sector strategies and programs, particularly in the education and health sectors, as set forth below.

\subsubsection{Education and training}

326. The educational strategy seeks to attain the Millennium Development Goals (MDGs), namely to raise the primary school access and completion rates to 100 percent by 2015 and to improve the girl/boy parity index to 1 over the same period. 


\begin{tabular}{|c|c|c|c|c|c|}
\hline \multirow[t]{2}{*}{ MDG } & \multirow[t]{2}{*}{ Progress evaluation indicators } & \multicolumn{2}{|c|}{ Current situation } & \multicolumn{2}{|c|}{ Goals } \\
\hline & & Level & Year & Level & Target year \\
\hline \multirow{3}{*}{$\begin{array}{l}\text { Ensure primary } \\
\text { education for all }\end{array}$} & $\begin{array}{l}\text { Net primary enrollment ratio } \\
\text { (ages 6-11) }\end{array}$ & 75.2 & 2001 & 100 & \\
\hline & Primary school access ratio & 90 & 2000 & 100 & 2015 \\
\hline & Primary school completion rate & 56 & 2003 & 100 & \\
\hline \multirow{4}{*}{$\begin{array}{l}\text { Promote gender } \\
\text { equality }\end{array}$} & Girl/boy parity index & 85 & 2000 & 100 & 2015 \\
\hline & $\begin{array}{l}\text { Secondary school girl/boy parity } \\
\text { index }\end{array}$ & 81.5 & 2000 & 100 & 2015 \\
\hline & $\begin{array}{l}\text { General secondary } \\
\text { education }\end{array}$ & 87.5 & & 100 & \\
\hline & $\begin{array}{l}\text { Technical secondary } \\
\text { education }\end{array}$ & 61.7 & & 100 & \\
\hline
\end{tabular}

327. The findings of the 2001 Cameroon Household Survey (ECAM II) and the information gathered during the participatory consultation highlighted the importance of education and technical and vocational training as a decisive factor in poverty reduction. This being so, the government adopted a sector strategy for education in October 2000, seeking to achieve the following objectives: (i) improve access to education while correcting disparities; (ii) increase the quality of education provided; (iii) develop efficient partnership with the private sector and civil society; and (iv) improve the management and governance of the education system.

328. The strategy is focused on: (i) universal primary education; (ii) improved access and equity at the other levels of education; (iii) improved quality and relevance of the curricula; and (iv) improved management and governance.

329. Early childhood (subprogram 3): The government intends to promote preschool education and to encourage initiatives in favor of its community-based development in order to prepare children for subsequent learning. To that end, the government would: (i) increase educational facilities, especially in rural areas; (ii) involve local communities, NGOs, communities, and families in the funding of preschool education; (iii) diversify nursery school premises coupled with integrated, flexible programs; and (iv) train teachers and conduct effective monitoring and supervision of the functioning of preschool facilities.

330. Primary education (subprogram 4): To make this level of education universal, the government will take measures to increase education supply and boost education demand, especially for girls, through: (i) the construction and rehabilitation of school buildings; and (ii) the recruitment of and in-service continuing education for teachers. The government also encourages the enrollment of all children of school age and, jointly with certain development partners (UNICEF, UNESCO, NGOs, etc.), will mount an awareness and information campaign sensitizing parents and communities on the benefits of educating all children. This will go in tandem with poverty reduction. 


\section{Figure 1. Objectives, Priorities, and Programs for the Education Strategy, by Sector}
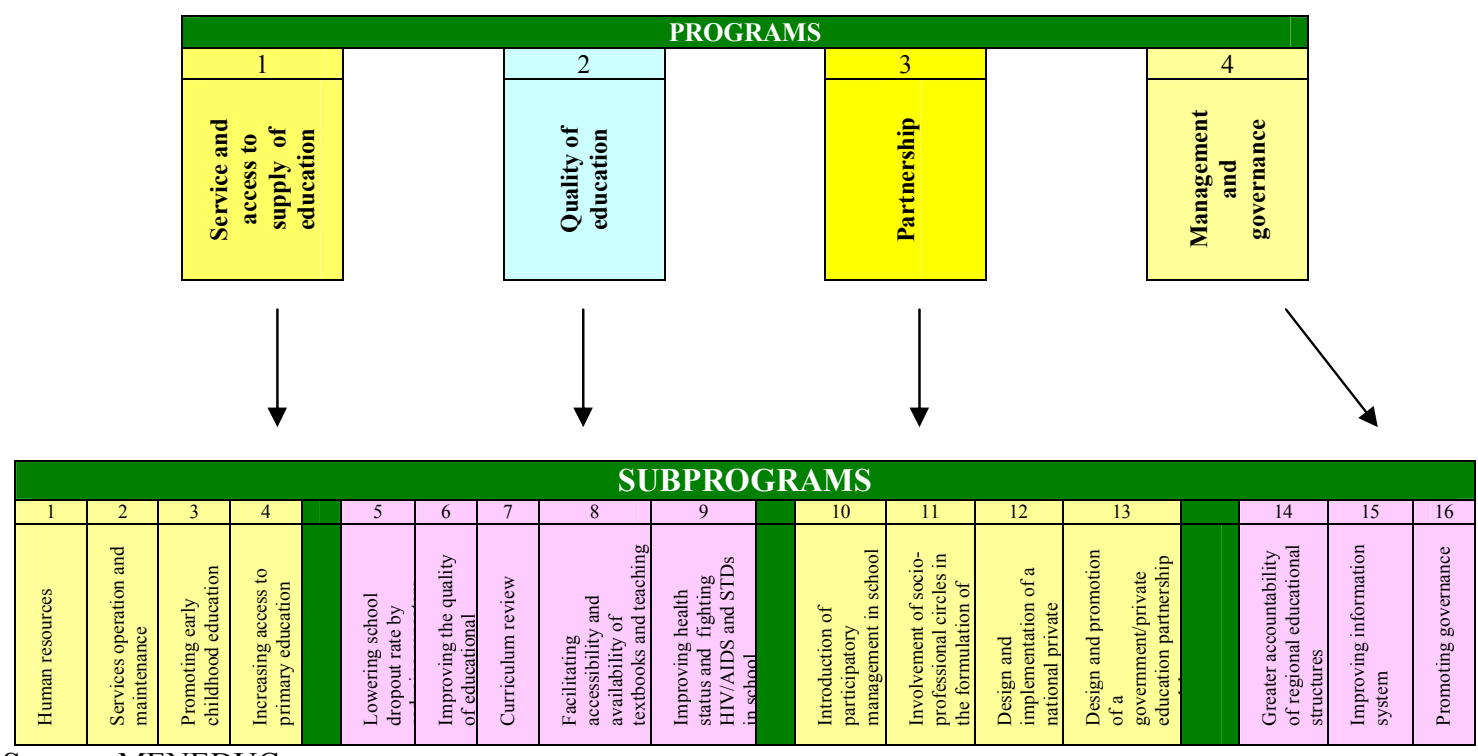

Source: MENEDUC

331. General secondary education (subprogram 5): The main measures to be adopted will focus on increasing the number of classrooms and recruiting a sufficient number of teachers. The measures will help to: (i) increase the number of admissions into the primary cycle; and (ii) consolidate the partnership with the private sector, notably with regard to the increase in the number of classrooms and the improved management of private institutions, within the framework of a Secondary Education Law currently under preparation.

332. Curtailment of the dropout rate (subprogram 6), improvement of the quality of teaching (subprogram 7), and curriculum review (subprogram 8): In 2001/02, as previously observed (paragraph 2.4.1), the survival rate at the end of primary education is 56 percent whereas the access level in the first grade is 95 percent. Repeaters account for 25 percent in primary education, 21 percent in the lower cycle of general secondary education, and 31 percent in the higher cycle of general secondary education. The initial financial projections established from the education policy choices currently implemented by the Ministry of National Education clearly show the long-term budget unsustainability of such a strategy. Aware of the limits of such a policy, the government of Cameroon is in the process of preparing new policies to enhance the effectiveness of education spending and still achieve MDG targets.

\section{Box 22. Financial Cost of the Status Quo Policy in the Education Sector}

The government devised a tool for performing simulations of the medium-term prospects for education and funding of the sector. The simulations show major budgetary imbalances in the absence of policy or educational measures to improve access, internal efficiency, the quality of instruction, and management and governance within the system. In this context, the cost of achieving the MDG objectives would call for additional funding on the order of CFAF 43 billion in 2003, and roughly CFAF 100 billion in 2011. Mobilizing funding to meet those needs would prove very difficult.

Source: MINEDUC. 
333. Among other things, these policies address reducing grade repetition rates and increasing the transition rate from primary to secondary education, with the set objective of lowering the repetition rate, as soon as practicable, to a threshold of 10 percent at each education level (compared to average rates of 25 percent in primary education, 21 percent in the first cycle of secondary education, and 31 percent in the second cycle of secondary education). This presupposes the implementation of "activist" policies in both administrative and pedagogical areas.

- In primary education, the authorities intend to review the evaluation and certification systems through the creation of three subcycles (SIL/ $\mathrm{Cl} 1+\mathrm{CP} / \mathrm{Cl} 2, \mathrm{CE} 1 / \mathrm{Cl} 3+\mathrm{CE} 2 /$ $\mathrm{Cl} 4, \mathrm{CM} 1 / \mathrm{Cl} 5+\mathrm{CM} 2 / \mathrm{Cl} 6 / \mathrm{Cl} 7)$. In this context, the content of curricula is structured over two years of study without any possibility of repeating two classes in the same subcycle. Teachers will be provided with educational tools for formative evaluation, to help them monitor the progress made by their students during the cycle and provide assistance to slow learners; for summary evaluation purposes, at the end of each subcycle a yardstick is applied so that access to the new subcycle may be properly regulated, based on relevant learning. The accompanying measures for this arrangement are: (i) revision of teaching methods; (ii) application of differentiated educational methods (remedial teaching and assistance to slow learners); (iii) systematizing the measurement of student educational achievements; (iv) reducing class size to a maximum of 50 students in the short term and to 47 by 2011; (v) assigning qualified, experienced teachers to classes in the first and last subcycles; (vi) the improvement of in-service continuing education for teachers; (vii) providing students and teachers with textbooks and instructional materials; and (viii) the formulation of a community-based early childhood development policy, etc.

- Secondary education under the Education Orientation Law provides for two cycles of studies (the first cycle is limited to 5 years, the first two years of which are devoted to observation, and a second two-year cycle: $1^{\text {ère }} /$ lower sixth and terminale/upper sixth). The authorities contemplate: (i) reinforcement of school counselling and guidance to help steer students with obvious skills for this type of education toward technical and vocational education; and (ii) increased accountability in schools in general, and in the education community at large, as part of the educational projects included in School Councils.

334. Technical and vocational education. Until 2002, the education strategy was characterized by the absence of a national development policy in the technical and vocational education subsector. In August 2002, a Ministry of Technical Education and Vocational Training was created. It is currently formulating a sector strategy to implement a national technical education and vocational training policy. Its key missions consist in:

- Producing, in addition to the school map, a mapping of existing investment opportunities in human capital based on proven potential for socioprofessional integration;

- Creating specific pilot technical and vocational training institutions by ecological zone, to take advantage of opportunities offered by the environment;

- Re-examining the training certificate award strategy with a view to establishing a partnership with professional societies, corporations, and guilds;

- Reorganizing the rural crafts/domestic science sections (SAR/SM) in order to foster a breeding ground for rural entrepreneurs and curb the rural exodus;

- Readjusting training programs and courses, in concert with socioprofessional circles, civil society, and national and foreign partners; 
- Formulating and implementing an advanced training plan for staff and workers;

- Conducting studies on training needs for the labor market;

- Building the capacity of existing structures by equipping the workshops in Technical Schools and Vocational Training Centers;

- Modernizing technical education and vocational training through the creation of highly efficient training institutions adapted to the needs of the regions;

- Raising public awareness, through diverse techniques including information, education and communication (IEC), of the role and importance of technical education and vocational training, in order to dispel the derogatory perception of technicians' skills.

335. Efficient partnership (subprograms 11 to 14), which is already reflected in the management of educational institutions and will be further developed, particularly in the areas of technical education, technology and vocational training, and the promotion of private education. To this end, government will mount an awareness, information, and training campaign among the various partners (parents, local communities, NGOs, religious denominations, trade unions, private promoters, development associations, donors, etc.), with a view to evolving a platform codifying the contribution of each partner in school projects in the framework of School Boards.

336. The government will build on the comparative advantages of the private sector to establish private educational institutions throughout the country to improve access to education and equity, and to enhance the quality and relevance of universal education. To this end, the government will strengthen the already existing cooperation with private promoters involved in education and training activities through the following measures: (i) simplification of procedures for founding private schools; (ii) making set tuition fees affordable to parents; (iii) offering incentives for the creation of private schools in rural areas; and (iv) broadening the scope of state intervention through multifaceted support ranging from subsidies for the transfer of teachers from public to private institutions to the improvement of educational infrastructure. In addition, the government will negotiate the debt incurred by the private education sector with the National Social Insurance Fund (Caisse Nationale de Prévoyance Sociale (CNPS)) and the Tax Administration, after prior auditing of the debt.

337. Management and governance (subprograms 15 to 17). The government will devote particular attention to promoting good governance in the educational sector, notably through the deconcentration and decentralization of resource management, participatory management, cost control, and institutional capacity building in the education sector. To this end, the competent authorities have issued directives including: (i) setting up a career development administration; (ii) transferring teachers and physical education/sports instructors' career management from the Civil Service Ministry to the Ministry of National Education; (iii) improving the information system in the Ministry of National Education by drawing up a school map and regularly publishing a statistical directory of the education sector; (iv) including statistics courses and school record-keeping as part of teacher-training programs; (v) ensuring the regular production of management statistics at all levels of the educational system; (vi) facilitating access to new information and communications technologies within the educational system; and (vii) reinforcing the supervisory and evaluation structures in the Ministry of National Education.

338. Higher education. Conscious of the role and place of higher education as a laboratory for "know how" and considering the spiraling number of students, the government created 
five additional state universities following the 1993 reform: Yaoundé (Soa), Douala, Buea, Dschang, and Ngaoundere, thus bringing the total number of state universities to six. The basic orientations of the reform are as follows:

- Participation of various partners in the management and funding of higher education;

- The greatest possible autonomy in academic matters and management;

- Equal access to higher education for all Cameroonians;

- Professionalism and increased education supply;

- Multidisciplinary and deconcentrated education;

- Opening up to the local, regional, national, and international environment;

- Efficient use of the existing infrastructure and available resources; revitalization of interuniversity and international cooperation.

339. Furthermore, in consultation with socioprofessional circles the authorities devised a regulatory framework leading to the founding of a number of private higher education institutions. On the basis of problems identified in the higher education and vocational training subsectors, the authorities plan jointly with the private sector and civil society to: (i) improve access to, and the quality and the relevance of instruction in, all the universities through the rehabilitation and construction of academic and social infrastructure, notably lecture rooms, auditoriums, university dormitories, tutorial and practical work rooms, laboratories, and libraries; (ii) set up a network built on information and communications technology in order to reinforce interuniversity and international exchanges; (iii) grant franchises for university services such as lodgings, restaurants, and transportation; (iv) develop programs adjusted to meeting the new needs of the labor market and facing the changes occurring in the modern world; and (v) control Sexually Transmitted Infections (STIs) and HIV/AIDS in universities. 


\section{Box 23. Sector Strategy for Education}

Economic recovery, which brought about an increase in the budgetary allocation to the education sector (23.1 percent of the state budget in 1999/2000), helped the government to adopt measures aimed at making this sector one of the engines of growth and poverty reduction. Thus, in 2000/ 2001 the gross enrollment ratio was estimated at around 99 percent (90 percent for girls) and the net ratio was around 78 percent (67 percent for girls). The school access rate was about 95 percent and the completion rate about 56 percent. Major efforts were made to mitigate the constraints associated with the demand for education. In addition, there is a slight increase in preschool, primary, and secondary school enrollment as well as a slight reduction in regional disparities in education.

Furthermore, a number of actions were taken and others are being implemented, notably:

- $\quad$ provisions for optional approaches (purchases, subsidies, etc.) under the national textbooks policy;

- $\quad$ providing educational materials to all public primary schools;

- $\quad$ STD and HIV/AIDS control campaign in schools;

- The elimination of school fees in public primary schools (measure in force since the beginning of the 2000/2001 academic year) and allocation to schools, at the beginning of each academic year, of the required minimum (minimum package) of instructional materials;

- construction and rehabilitation of classrooms in public primary and secondary schools, with focus on priority education zones (ZEPs);

- elimination of CEPER's monopoly in textbook publishing and distribution;

- creation of a Conseil National d'Agrément (National Approval Board) for textbooks and instructional material), whose members have been appointed;

- the launching (in order to build the 2,500 new classrooms required to reach the completion point for the HIPC Initiative) of construction of 1,145 classrooms under the Public Investment Budget (BIP) for FY00/01 and FY01/02; other HIPC-funded construction is planned;

- recruitment of 1, 260 temporary teachers (IVAC) under HIPC funding during FY01/02;

- integration of 1,700 temporary teachers into the civil service in the Ministry of National Education;

- formulation, with a view to multifaceted partnership with private education, of draft laws and decrees and implementing provisions relating to this category of education. Finalization of the draft laws will require detailed analysis during a consultative workshop prior to introduction in the official channels for adoption, signing, and promulgation;

- formulation and publication of a statistical directory for $2000 / 01$ and paving the way for the systematic local production of school statistics on a decentralized basis, followed by the production of provincial and national school maps;

- formulation and signing of legislation on the implementation of the special status of the teaching profession;

- formulation and signing of a new organizational chart for the Ministry of National Education, providing for decentralized staff management. Henceforth, career management, postings, and promotions, for instance, will be conducted at provincial level. Implementation of the new arrangements is being carried out under the SIGIPES program.

- distribution of textbooks to students and teachers in priority education areas;

- allocation of generic drugs and first aid products to all public and private primary as well as public secondary schools;

- mounting of awareness and information campaigns to promote enrollment of large numbers of children, with particular emphasis on the ZEPs.

Several other measures are helping to promote the sector revitalization program. These include: (i) measures to reduce regional disparities and gender-based discrimination in access to school; (ii) provision for devolution of responsibilities to the communities (participatory management of schools/institutions); (iv) the construction of water supply points and latrines in schools; (v) control of STIs and HIV/AIDS in the school environment; and (vi) control of training costs, etc.

Source: MINEDUC

340. The government also plans to: (i) develop continuing and cooperative vocational education in all state universities through increased financing for higher education, diversification of learning opportunities, and the emergence of new technologies; (ii) develop, strengthen, and improve vocational training by taking the needs of companies into account; (iii) promote prospecting and the development of transfer and career enhancement activities; 
(iv) train and recruit highly qualified teachers and provide them with adequate educational tools and techniques in order to reduce migration to foreign universities; and (v) mobilize private funding in the higher education sector, through incentives.

\subsubsection{Health}

341. As indicated in the following table, the key health indicators of Cameroon deteriorated between 1991 and 1998.

\begin{tabular}{|lccc|}
\hline \multicolumn{4}{|c|}{ Table 19 : Target Level in $\mathbf{2 0 1 5}$ for Key Health Indicators } \\
\hline INDICATORS & $\mathbf{1 9 9 1}$ & $\mathbf{1 9 9 8}$ & $\mathbf{2 0 1 5}$ \\
\hline Neonatal mortality rate (per1,000) & 31.1 & 37.2 & 10.4 \\
Infant mortality rate (per 1,000) & 65 & 77 & 21.7 \\
Child mortality rate (per 1,000) & 65.6 & 79.9 & 21.9 \\
Infant-child mortality rate (per 1,000) & 126.3 & 150.7 & 42.1 \\
Malnutrition rate (12-23 months) (\%) & 32 & 44 & 16.0 \\
Maternal mortality rate (per 100,000 live births) & 430 & 430 & 107.5 \\
\hline Source: EDS 1991 and 1998; Millennium Summit documents. & & & \\
\hline
\end{tabular}

342. Therefore, improving the health of the population is certainly a key objective for economic and social development and for poverty reduction. The government expects to do so by implementing the Health Sector Strategy adopted in October 2001.

343. In the context of its efforts to better control diseases, the government of Cameroon intends to reduce the overall morbidity burden attributable to the communicable diseases that so far have been highly prevalent throughout the country. Most of these diseases are preventable through an effective policy combining prevention, information, and treatment. This policy will help fill the wide gap in economic growth resulting from a heavy morbidity burden due particularly to infectious diseases (malaria, HIV/AIDS, tuberculosis, onchocerciasis etc.) commonly known as diseases of poverty. Controlling these diseases will unleash the productive potential of labor and thereby extend the range of productive opportunities, significantly raising the rate of economic growth.

344. Combating malaria. The government has restructured the National Malaria Control Program by creating the Central Technical Group (GTC) with a Permanent Secretariat as well as provincial units to combat this disease. The program has acquired 150,000 mosquito nets and an equal number of insecticides using HIPC funding. Distribution of these mosquito nets and insecticides to pregnant women began in early 2003, and the Ministry of Health will acquire 660,000 additional mosquito nets and insecticides during the year. 


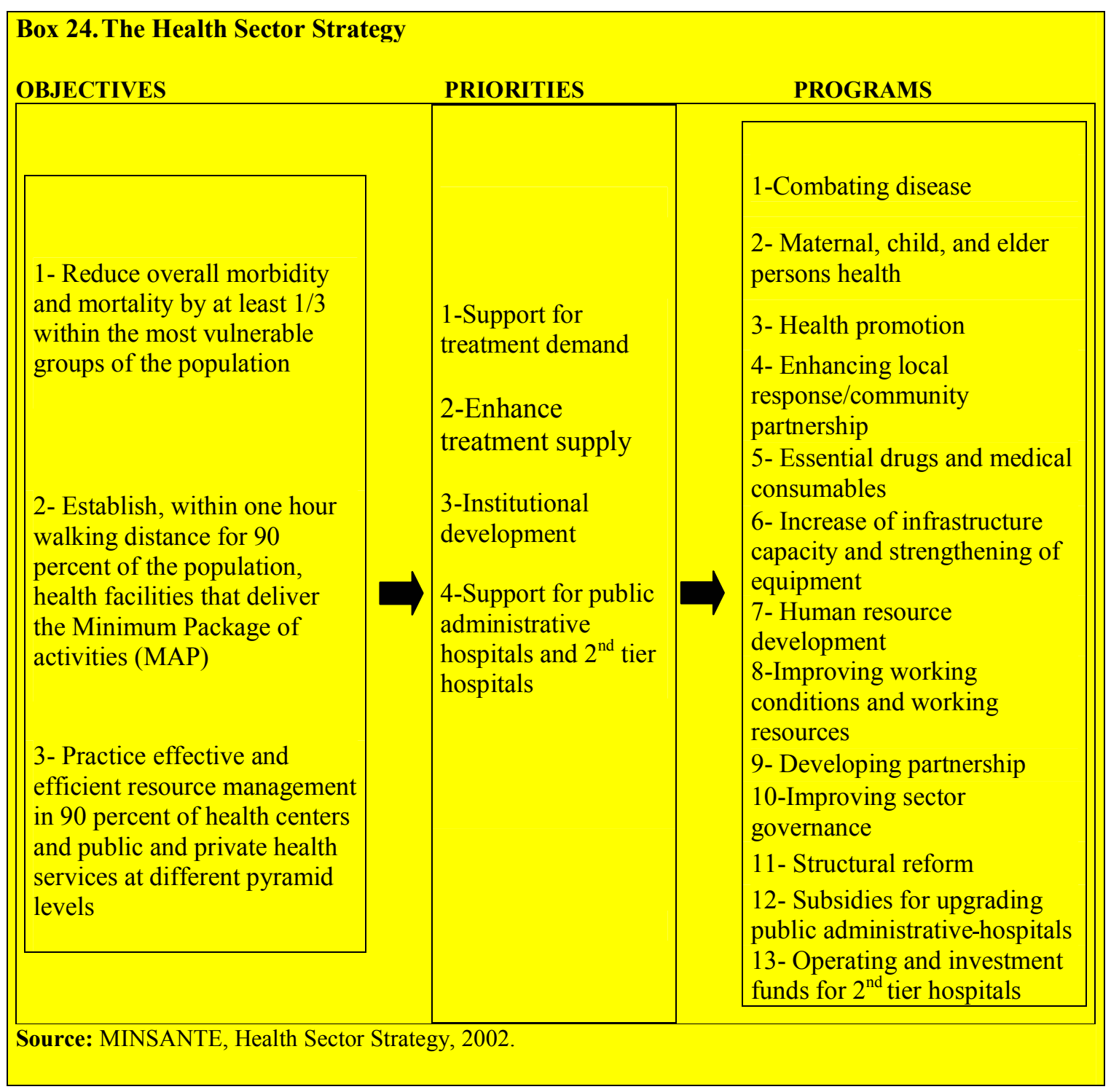

345. On the other hand, the government has adopted a National Anti-Malaria Strategic Plan in line with the "Roll Back Malaria" Initiative, which aims to halve malaria-related morbidity and mortality by the year 2010, particularly among the most vulnerable segments of the population (children under 5 and pregnant women). To this end, the government plans to: (i) improve the quality of care and treatment; (ii) intensify vector control; (iii) train personnel; (iv) promote malaria control; (v) develop partnerships; (vi) strengthen operational research; (vii) conduct integrated epidemiological surveillance; and (viii) set up supervision, monitoring, and evaluation mechanisms.

346. Combating STIs and HIV/AIDS. The government has effectively integrated AIDS drugs (ARVs and medicine for treating opportunistic infections) into the national supply and distribution system for essential drugs, reagents, and medical supplies. It also subsidizes antiretroviral drugs (ARVs), whose monthly cost for an individual ranges between CFAF 15,000 and CFAF 28,000 since August 1, 2002 in both the public and private health sector. The national HIV/AIDS control program procures condoms for target groups. Since the beginning of 2003, their distribution has been contracted out to NGOs through partnership 
agreements. The government has also decentralized the fight against AIDS by establishing an operational Provincial Technical Group (GTP) in each province.

347. The government will also continue implementing the 2000/2005 Anti-AIDS Strategic Plan adopted in September 2000. Its implementation will entail: (i) developing a communication plan involving the public and private media; (ii) raising awareness among youths in schools, at universities, and out of school, women, public and private workers, and persons living in rural areas; (iii) promoting the use of male and female condoms within target populations; (iv) encouraging voluntary testing; (v) creating anonymous prevention and voluntary testing centers in each health district; (vi) extending the national program for reducing mother-to-child HIV transmission; (vii) community participation through dialogue structures and associations; (viii) developing mechanisms for the medical and psychosocial care of people living with HIV/AIDS (PLWHA); (ix) developing a solidarity system for PLWHA; (x) developing and strengthening partnerships (public, private, secular, etc.); (xi) strengthening epidemiological and behavioral surveillance; (xii) capacity building; and (xiii) expanding research.

348. Fighting Tuberculosis. The government has restructured the National Tuberculosis Control Program by creating a GTC with a permanent Secretariat and provincial units. The program has used HIPC funds to acquire drugs. The government has been able to reduce the cost of treatment from an average of CFAF 30,000 to CFAF 5,000 per month. It has also formulated a National Tuberculosis Control Program covering all the 10 provinces, which will be adopted soon. When this plan is implemented, the government will: (i) create a Diagnosis and Treatment Center (DTC) for every 50,000 to 100,000 inhabitants by using the "DOTS" strategy; (ii) enhance technical supervision by involving provincial hospitals; and (iii) build the capacities of other health workers outside DTCs to effectively handle cases.

349. Fighting onchocerciasis. The government will continue implementing the action against onchocerciasis in partnership with NGOs and other partners using the following strategies: (i) distribution of Ivermectine through communities to all persons eligible for treatment in hyper- and meso-endemic zones; (ii) passive distribution of Ivermectine in hypo-endemic zones; (iii) training of staff involved in onchocerciasis control; (iv) development of operational research; and (v) information, education, and communication targeting the population at risk.

350. The Expanded Program on Immunization (EPI). The authorities reorganized this program by creating a Central Technical Group (GTC) with a permanent Secretariat. They will also implement strategies contained in the five-year EPI plan of action centered on: (i) the mobilization of additional resources within the framework of vaccination independence (GAVI); (ii) basic training and in-service training for the health personnel responsible for implementation; (iii) the rehabilitation/renewal of equipment, vehicles, and the cold chain; (iv) development of communication for behavior change; (v) entering into contracts with health districts; (vi) implementing an injection safety policy; (vii) introducing new vaccines such as those against yellow fever and hepatitis B; (viii) strengthening the monitoring and evaluation of program activities; (ix) strengthening EPI management at all levels; and (x) enhancing epidemiological surveillance.

351. Essential drugs, reagents, and medical supplies. The government will improve access to such items by the population by making them available at all health facilities. The government has already reduced the prices of essential drugs, reagents, and supplies by about 
40 percent to make them affordable to the population. A substantial supply of essential drugs, acquired under the HIPC program, will help improve their availability at health facilities.

352. To attain its main objectives in the sector, the government will implement the National Pharmaceutical Master Plan by: (i) developing an effective national system for the supply of drugs, reagents, and medical consumables (the SYNAME), with central facilities and branches with decentralized management; (ii) organizing an operational system to ensure the quality of medical tests and pharmaceutical products; (iii) organizing a selection system for pharmaceutical products, preferably under their common international denomination; (iv) developing pharmaceutical research; (v) establishing a pharmaceutical monitoring system; (vi) subsidizing selected expensive therapies and medical tests; and (vii) facilitating private sector access to generic drugs, etc.

353. Emergencies, epidemics, and disasters. The government will establish effective care mechanisms focused on the following strategies: (i) the development of effective epidemiological surveillance sentinel posts for rapid decision-making and adequate response; (ii) staff training at all relevant levels; (iii) the development of emergency operational networks; (iv) information, education, and communication interventions; and (v) operational research.

354. Financing health care. The government will take appropriate measures to help cover the cost of treatments by instituting a health risk-sharing system through the development of health mutual benefit associations. In this connection, a support unit for the development of health mutual benefit associations has been created at the Ministry of Public Health, with the mandate to: (i) draft a mutual health insurance code; (ii) coordinate the stakeholders involved in supporting mutual health insurance; and (iii) undertake a pilot study on the development of community-level health mutual benefit associations.

355. Maternal, adolescent, and elderperson health. The government will implement the following strategies to effectively address the health problems of these groups: (i) developing a minimum package of activities in the form of curative, preventive, and proactive care at each level of the health pyramid; (ii) promotion of reproductive health adapted to the needs of adolescents/youths; (iii) basic and in-service training for all health care providers; (iv) enhancing operational research in reproductive health; (v) enhancing the health information system for program management; and (vi) monitoring and evaluating reproductive health activities.

356. Food and nutrition focused on the child. With the support of development partners, the government will implement programs which, by 2011, will help to: (i) halve protein-energetic malnutrition among children under 5; (ii) reduce anemia by one-third among women of childbearing age and preschool children; (iii) eradicate vitamin A deficiency among children under 5; and (iv) eradicate iodine deficiency-related difficulties.

357. To achieve this objective, the Cameroonian authorities will strive to: (i) encourage and support breast-feeding as well as better infant nutrition practices; (ii) improve the policy of systematically administering iron and folic acid supplements to pregnant women and promote the production and consumption of iron-rich local food products; (iii) administer Vitamin A supplements to all women who delivered and to children aged 6 months to 5 years; and (iv) promote the consumption of iodine-enriched salt in all households, etc. 
358. Noncommunicable diseases. Some noncommunicable diseases constitute major public health problems. Accordingly, the government intends to strengthen the noncommunicable diseases program by implementing the following measures: (i) developing an operational epidemiological surveillance system for noncommunicable diseases; (ii) developing systematic screening for breast, cervical, and prostate cancer; and (iii) developing screening for diabetes, high blood pressure, and sickle cell anemia. 


\section{Box 25. Fighting AIDS, a Government Priority}

According to the information available, the first 21 cases of AIDS were diagnosed and reported in Cameroon in 1986. Subsequently, the epidemic spread rapidly among the sexually active population. In 1999, the total number of new cases stood at 20,419 cases. The prevalence rate rose from 0.5 percent in 1987 to 2.1 percent in $1991,3.8$ percent in 1998, and 11 percent in 2000.

Analysis according to sex and age indicates that (i) youths are most affected with 43 percent of the seropositive individuals being between age 15 and 35; (ii) women are more affected than men by a $3: 2$ ratio; and (iii) transmission is predominantly heterosexual, with 90 percent of infections occurring during unprotected sexual intercourse and 10 percent resulting from blood transfusion and mother-to-child infection.

According to UNAIDS estimates, of the 540,000 Cameroonians living with HIV/AIDS (PLWHA) in 1999, some 290,000 were women aged 15 to 49 , while 22,000 were children aged 0 to 14 . Projections indicate that $1,500,000$ Cameroonians lived with HIV/AIDS in 2001 and that more than 150,000 had been orphaned by the disease.

The epidemiological situation is marked by a rapid increase in seropositivity and in people with full-blown AIDS, particularly among youths, women, the armed forces, truckers, and sex workers.

The senior government leadership became involved in the fight against HIV/AIDS early on through: (i) creation of a committee to combat AIDS and the national AIDS control program in 1986; (ii) formulation of a short-term program against AIDS in 1987; and (iii) drawing up two medium-term plans against AIDS covering the 1988-92 and 1993-95 periods, and the preparation of a framework plan against AIDS for the 1998/2000 period. No satisfactory results were obtained through these programs.

In September 2000, the government adopted a strategic plan to combat AIDS for the 2000-05 period. By means of this plan, the authorities expect to reverse the epidemic's current trend, reduce seropositivity to less than 10 percent, and cut the incidence of HIV by 25 percent among youths, men in uniform, and women between now and 2005 .

The strategic plan has been implemented within a context of a new and improved program management, a multisectoral approach, and the decentralization of intervention. It is aimed at encouraging: (i) future generations of Cameroonians aged 5-14 to adopt a healthy life style in the HIV/AIDS era; (ii) adults to understand that they live in a world with HIV/AIDS and must adopt responsible sexual behaviors; and (iii) Cameroonian society to revive basic solidarity in support of persons living with HIV/AIDS. The outcome of the implementation process has been to:

- Broaden the national response through (i) the development of sector plan by the public administration (the Ministries of Defense, National Education, Youth and Sports, Territorial Administration and Decentralization, Women's Conditions, Social Affairs, and Higher Education); (ii) the signing of agreements with religious groups and private sector enterprises to wage the battle against HIV/AIDS within these communities, especially through preventive education, the promotion of condom use, and care for AIDS patients;

- Enhance local response by setting up a process involving local authorities and communities by creating local NGOs;

- Strengthen and organize program management through: (i) the creation of a joint monitoring commission, which meets regularly; (ii) the creation of a multidisciplinary central management team; and (iii) capacity building and improved logistical support for the central management team, as well the identification of offices for that team;

- Reduce the price of antiretroviral drugs, the cost of which ranges has ranged between CFAF 15,000 and CFAF 28,000 per month and patient since August 1, 2002.

The government is committed to the program, and its financial support has risen continually since 1986 from CFAF 53 million in 1986 to slightly over CFAF 1.6 billion in 2000. Donor willingness to support the program has also increased. Of the CFAF 213 billion in HIPC resources over the 2000-2003 interim period, the AIDS Control Program has received CFAF 5.4 billion with a view to accelerating the implementation of the following interventions: (i) 100 percent condom use; (ii) counselling and voluntary testing; and (iii) behavioral changes among youth.

Source: MINSANTE.

\subsubsection{The urban poverty reduction strategy}

359. Rural exodus resulted in the accelerated and unplanned urbanization observed over the past two decades coupled with economic crisis. Cameroon's cities, particularly the largest of 
them, are facing a number of teething problems such as explosive shanty towns, the lack of sanitation, the inaccessibility of certain areas, insecurity, unemployment, and increased organized crime. With urban areas growing at an average of 5 percent per year (7 percent for Yaoundé and 6.4 percent for Douala), nearly 60 percent of the Cameroonian population is expected to be urban-based around 2015.

360. Rapid urbanization brings the major challenge of accommodating an increasingly young population. The Cameroonian population, like that of many other African countries, is relatively youthful, with an average age of 22 years; nearly 42 percent of Cameroonians are under 14 and more than two-thirds are under 30 . As is the case everywhere, this population tends to concentrate in urban areas, resulting in increased pressure on social services, infrastructures, and labor markets. This is a situation that calls for heightened and sustained attention by all.

361. The government of Cameroon is well aware of the extent of the problems and is in the process of drawing an integrated urban development policy. Its objectives are to: (i) improve the living conditions of urban dwellers, a majority of whom live under precarious conditions; and (ii) reinforce the economic role of towns by strengthening urban infrastructures (extension, rehabilitation, and maintenance). This will not only improve living conditions, but will also and especially support industrial growth and services and help to integrate youths, women and other groups into economic channels.

362. Although the strategy is still at a conceptual level, short-term actions are already underway to address the most pressing problems. They include:

- the rehabilitation of basic infrastructures (road maintenance and street lighting);

- opening up poorly served areas and reorganizing squatting areas;

- the development of urban extension zones and secondary urban centers;

- sanitation (basic drainage system, rehabilitation of storm water purification plants);

- the management of household and industrial waste (building garbage dumps, construction of public latrines, removal of pieces of wreckage, etc.);

- low-cost housing;

- attending to the needs of street children and mentally deranged persons;

- sensitizing prostitutes on the risks of sexually transmitted infections (STIs) and HIV/AIDS;

- combating insecurity.

363. The development of a low-cost housing promotion program in urban areas constitutes one of the government's major priorities in its poverty alleviation strategy policies. In 2003, the government will initiate a study on the preparation of a national housing policy in order to establish an appropriate sector strategy. The study should help: (i) establish an adequate regulatory and institutional framework; (ii) clarify the respective roles of the state (including decentralized territorial communities), the housing agencies such as MAETUR, a public corporation responsible for planning urban areas and construction in rural and urban sites in Cameroon, the Société immobilière $d u$ Cameroun (SIC), a mixed capital corporation responsible for the construction of quality housing and the management of the state property of Cameroon, Crédit foncier du Caméroun (CFC), a housing finance institution, and the private sector (iii) identify incentives for this kind of program without prejudice to other initiatives and (iv) identify sources of funding for the program. The government has 
established a pilot project for the construction of model houses to meet the important and pressing needs of the cities of Douala and Yaoundé, to be financed by the CFC.

364. The government will continue to encourage partnership agreements between the state and decentralized territorial communities to increase the participation of local actors in improved town management. Steps will be taken to strengthen the capacities of various urban stakeholders with the prospect of participatory management in operational, monitoring, and appraisal activities. This will entail improving the intervention capacities of technical units and enhancing control over works, particularly in the urban communities of Douala and Yaoundé.

365. In the urban transportation sector, the government has decided to allow private transportation companies to operate in the cities of Yaoundé and Douala on the network of the former Urban Transport Company of Cameroon (SOTUC). Invitations to bid were launched. In Douala, the selected company, SOCATUR, has provided residents with a service that has been appreciated although its fleet is still inadequate. In Yaoundé, however, the operator selected failed to fulfill the conditions and new bids have been invited. Pending the completion of the process, the government has issued temporary licenses to several private bus owners to provide a service on some of the lines of the Yaoundé urban network, with the objective of improving local transportation services for the poorest and most vulnerable by increasing supply.

\subsubsection{Other social development policies and strategies}

366. Participatory studies have revealed that socially dysfunctional behaviors such as the social exclusion of certain categories of persons and the lack of consideration they have to endure are yet another dimension of poverty. The government, with the support of various partners, wants to respond to this concern and to mitigate inequalities and the exclusion of vulnerable social groups. It will therefore establish an institutional, legal, and regulatory framework guaranteeing the rights and social protection of these groups.

367. With this in mind, the government intends to produce its Social Development Strategy in 2003. This document will define short- and medium-term actions in the areas of: (i) social protection and national solidarity; (ii) the promotion of gender equity and equality; (iii) social education; (iv) employment; and (v) the satisfaction of basic needs.

368. The government also intends to: (i) finalize the Loi portant code des personnes et de la famille, an Individual and Family Rights and Protection Act; (ii) draft a law on social protection of the child; (iii) amend Law 83/013 of July 21, 1983 on the protection of the disabled; and (iv) enact a law on the social integration of the population.

369. Infrastructure and mechanisms for social integration. The government will take measures aimed at encouraging the creation of socio-collective structures and provision of social services and programs adapted to the specific conditions of the socially disadvantaged. Consequently, it will take appropriate measures for: (i) the rehabilitation of social centers and social action services; (ii) the development of social integration social services; and (iii) the promotion of mutual aid networks and social community actions. To that end, it will encourage the creation of a Special Decentralized Fund, financed using its own resources and other resources from the HIPC Initiative, official development assistance, and the C2D, a 
French debt reduction initiative. A feasibility study on the creation of the Fund will be conducted in order to determine its operating methods and systems.

370. Social reintegration. Steps will be taken to develop effective reception facilities, promote rehabilitation, and improve the provision of social services for socioeconomic integration. To this end, the following will be carried out: (i) the restoration of training institutions for the disabled; (ii) the diversification of effective rehabilitation facilities depending on the type of disability; (iii) the promotion of community action networks and alternative services for the disabled; and (iv) the development of social integration services for the socioeconomic integration of the handicapped.

371. The efforts of the government and donors will also be focusing on improving conditions for the special protection of children that are in particularly difficult situations. Specific measures to attain that objective include: (i) restoring rehabilitation institutions for minors who are social misfits; (ii) opening transit and reception centers to attend to street children and minors victimized by various forms of trafficking and exploitation; (iii) caring for AIDS orphans; (iv) improving social services for supervising children who are in conflict with the law; and (v) promoting special protection, deviance prevention, and open centers for the rehabilitation of youthful offenders.

372. The government will also take measures aimed at maintaining family stability and promoting responsible parenthood. Emphasis will be placed on: (i) the harmonization of family law by finalizing the individual and family rights code; (ii) the development of family educational programs for responsible parenthood and the social component of the fight against HIV/AIDS; and (iii) the implementation of family psycho-social support programs and the development of accompanying local mutual aid and training initiatives.

373. Women. The government will continue its efforts to improve living conditions for women, respect for their rights, the effective recognition of their contribution to development, and their integration into income-generating economic activities. For this purpose, the government will produce before December 2003 a Women Development Strategy. Its priorities are: (i) enhancing the socio-legal status of women; (ii) improving women's living conditions; (iii) promoting gender equality and equity in all sectors of national life; and (iv) enhancing structures and institutional mechanisms.

374. The plan of actions aimed at enhancing the socio-legal status of women would lead to less women being the victims of violence and discriminatory practices, and to a better protection of women's rights and dignity. It includes: (i) heightening awareness of the legal provisions on the rights and duties of women in Cameroon; (ii) improving women's access to justice; and (iii) disseminating and enforcing national and international legal instruments for the development and well-being of women.

375. Specific priorities in improving women's living conditions include: (i) strengthening women's economic power, with a focus on rural women; (ii) eradicating gender disparities in the primary and secondary school enrollment of girls; (iii) introducing incentives to encourage girls to opt for science and industrial technical subjects; (iv) facilitating the access of women of childbearing age and adolescents to reproductive health services and information; and (v) promoting appropriate technologies to reduce the workload of women. 
376. Steps will be taken to: (i) encourage women's access to loans and inputs and promote women's entrepreneurship; (ii) eradicate biases and discrimination in the school enrollment of young girls, and promote civic, economic, political, moral, and legal education for women and young girls; (iii) make the general public, and women in particular, more aware of the importance of environmental sanitation to health; and (iv) enhance women's knowledge of family planning and popularize responsible parenthood education, etc.

377. The promotion of gender equality and equity in all sectors of national life will help to mainstream the gender approach in development projects and build the capacities of female development professionals as regards the gender approach. The government intends to achieve this through a wide range of actions including advocacy, institutional mechanisms for the coordination of the gender approach in development activities, the training of decision makers, planners, and personnel from the ministries dealing with women-related issues, and the publication of practical manual for the popularization of gender issues, etc.

378. To improve existing structures and institutional mechanisms, the government intends to: (i) establish an autonomous body for the collection, pooling, analysis, and distribution of data and information on the situation of women; (ii) improve the intervention capacity of field workers in the promotion of women; and (iii) build the capacity of decentralized or specialized ministry departments dealing with women's affairs, especially women's development centers and centers for appropriate technology.

379. Social protection. The government plans to reform the social protection system to ensure that it covers socio-professional categories that are still not included. To this end, it will: (i) extend social protection to other population categories, notably the informal sector, rural dwellers, and self-employed professionals, tradesmen, and artisans; (ii) improve existing benefits, notably pensions, family allowances, and professional risks to adapt them to the current socioeconomic context and international norms; and (iii) create new institutions to ensure rigorous management and laws to pave the way for reforms.

380. The government has launched ten studies in order to ensure the success of its reform of the social security system. It is determined to use the findings of these studies to select key priorities to guide the reform strategy, in particular improving knowledge on: (i) the harmonization of the benefits level for all categories of beneficiaries so as to reduce social inequalities; (ii) separate management of branches and the creation of autonomous funds to ensure the sustainability of systems and avert the shifting of deficits from one branch to another; (iii) the private management of social security funds to improve administration and security; (iv) an increased presence in the highest employer and employee management organs which contribute to the social security regime; (v) the adoption of a mixed system including an allocation scheme and single premium method to better mobilize savings and ensure substantial revenues, sustaining the system while maintaining the principle of solidarity for the most disadvantaged; and (vi) redefinition of the role of the state.

\subsubsection{Job creation and the integration of disadvantaged groups into the economy}

381. The government considers the promotion of income-generating activities, especially selfemployment among the poor, as an appropriate and sustainable response to the problem of poverty. This in turn requires both improving the capacity of the poor to generate sufficient income to meet their basic needs and providing sustainable financing to essential social services. 
382. The government will encourage the production of goods and services primarily required by the poor, ensuring that its assistance targets financially and economically sustainable activities. In this connection, it will support the initiatives of the poor in the most promising sectors such as food crop production, agroprocessing, small-scale livestock, and counter season crops.

383. In the job creation strategy it is currently preparing, the government will: (i) finalize and adopt the National Employment Policy Statement (PNE); (ii) establish information systems on the labor market; (iii) take measures for the development of SMEs/SMIs; (iv) conduct a study on employment in the informal sector; (v) take steps to promote labor-intensive activities (identified under the French acronym HIMO); and (vi) step up the fight against HIV/AIDS within professional circles.

384. Creating job opportunities and income-generating activities for the poor will require:

- building the capacity of the poor to generate sufficient income for their basic needs;

- simplifying administrative procedures to help informal sector businesses create jobs and income-generating microprojects. 


\section{Box 26. Status of the Rural Employment Development Support Program}

\section{Situation of program}

For more than two decades, the development of activities in the informal economy sector has been used as a strategy to combat unemployment. Most of these activities are found in rural areas and are primarily related to agriculture, livestock, and crafts. That is why FNE, as part of its mission of creating employment and combating poverty, has for a decade supported rural activities by offering the stakeholders training as well as material and financial resources for more profitable production through the Rural Employment Development Program (PADER).

\section{2 assessment report}

Since the launch of PADER in 1996, 15,634 rural dwellers have been trained and become self-employed in various rural activities at a cost of about CFAF 3.3 billion, including CFAF 1.75 billion for training and CFAF 1.55 billion for self-employment. Targeting more than 20 different products, these projects located in more than 50 villages have enabled self-employed people to achieve an average business turnover of CFAF 1,500,000 and a net profit of about CFAF 500,000 per annum.

The objective of the program in 2003 is to train and provide self-employment to 1,926 people of both sexes interested in working in the following subsectors and willing to live in the following localities:

\begin{tabular}{lclc}
\hline Product/region & $\begin{array}{c}\text { Number of } \\
\text { developers }\end{array}$ & Product/region & $\begin{array}{c}\text { Number of } \\
\text { developers }\end{array}$ \\
\hline 1. Rice & $\mathbf{8 0 0}$ & $\mathbf{2 .}$ Dry beans \\
Maga (Far North) & 200 & Tignere (Adamaoua) \\
Bandounga (West) & 200 & Batoum (West) \\
Modelle-Banakuma \& Ndop (Northwest) & 300 & Nyokon \& Ngwaya (Central) \\
Ndikimeki (Central) & 100 & Nkombou,Nkienegang \& \\
& & Ngwaya (West) \\
\hline$\quad$ Maize & $\mathbf{2 0 0}$ & $\mathbf{4 .}$ Garlic \\
3. & 75 & Bakong-Bangoulap (West) \\
Wovia \& Bota (Southwest) & 50 & Mokom (Far North) \\
Nsimalen (Central) & 75 & \\
Garoua Boulai (East) & & \\
\hline$\quad$ Tiles and earth brick making & $\mathbf{2 6}$ & \\
5. & 26 & \\
Ebolowa (South) & & \\
\hline Source: FNE. &
\end{tabular}

Source: FNE.

The project amount of CFAF 713,925,900 will be funded as follows:

FNE funds (launching, monitoring, and evaluation) $\quad 19,975,900$

Project developers' contributions $220,550,000$

HIPC Funds $\quad 473,400,000$

It is expected to generate CFAF $1,723,250$ in income, CFAF $812,371,000$ CFAF in profits, and more than CFAF $1,200,000,000$ CFAF in net income.

\subsection{IMPROVING GOVERNANCE}

\subsubsection{Improving governance toward greater population welfare}

385. As discussed in the preceding chapters, improving the welfare of all is the overarching objective of government's growth and poverty reduction strategy. The strategy rests on stimulating private initiative to make it the driving force of the economy and a major vector for the social distribution of wealth, and on making the ordinary citizen the ultimate actor and beneficiary of development. Therefore, the role of the government is to create a physical and institutional environment that is conducive to developing private initiatives and to improving the well-being of citizens. Governance capacities - in terms of institutions, management, and 
decision-making procedures - will need to be built in order to help the government carry out the following key functions:

- Providing strategic management of development;

- Enhancing the participation and mobilization of active forces;

- Enforcing the rule of law and ensuring the security of goods and persons;

- Managing public funds;

- Fighting corruption;

- Reforming the procurement process;

- Managing human resources; and

- Informing citizens.

386. Keeping this in mind, by 1996 the government of Cameroon had begun to prepare a National Governance and Corruption Prevention Program (PNG), the full implementation of which started in the second half of 2000. Feedback from the population during the participatory consultations points to the need for such a program.

\section{Box 27. National Governance Program: Objectives and Main Priorities of the Priority Action Plan}

In June 2000, the Head of State approved the National Governance Program (PNG) that was prepared through a participatory process involving all active forces within Cameroonian society with a view to achieving the following strategic objectives:

- Support sustainable growth and development;

- Reduce the poverty and social exclusion which affect a large part of the population;

- Build capacities for major actors, notably the government, private sector, civil society, media, and decentralized territorial communities;

- $\quad$ Promote government/private sector/civil society partnership;

- Strengthen the rule of law to better protect human rights; reform the judicial system;

- Introduce a real culture of accountability in public affairs management; and

- Improve transparency in the functioning of government apparatus; combat corruption.

To ensure effective implementation of the PNG, in August 2000 the government adopted a Priority Action Plan to improve governance and fight corruption. Actions are grouped into the following seven priority areas:

- Pursuing improved tracking of public expenditure;

- $\quad$ Strengthening social sector management, notably education and health;

- In-depth reform of procurement procedures;

- Enforcing the rule of law and ensuring the legal and judicial security of investments;

- Improving citizens' information on public management;

- Intensifying the combat against corruption; and

- Identifying and executing community-level pilot programs, to be managed by local governments.

\subsubsection{Strategic development management}

387. In an open and global economy, with increased competition and a rapidly changing world context, the government has to provide clear medium- and long-term directions to citizens and investors so as to improve visibility and predictability, and to make the environment more secure. To this end, the government of Cameroon will build public capacity in:

- Forecasting and defining national development priorities;

- Planning and implementing economy-wide and sector policies; 
- Developing strategic initiatives in priority sectors;

- Programming and monitoring of infrastructure, land planning, and housing development projects;

- Improving the efficiency of market operations;

- Designing human resources development policies;

- Preparing sector framework laws and programs;

- Building the capacities of various economic actors;

- Implementing an incentive system;

- Designing and implementing decent employment policies;

- Building capacities for regulatory agencies;

- Assessing the implementation of public policies (privatization programs, sector policies, etc.).

\subsubsection{Motivating and mobilizing Cameroon's active forces around a common vision}

388. Economic and social development, and therefore poverty reduction, requires the mobilization and involvement of all the social partners, including the most disadvantaged or vulnerable groups, in identifying and solving problems, as this will create a climate of confidence between government and civil society, as well as broad agreement on and support for the government's overall policy stance. The government must stimulate a dynamic participation with the broadest representation of society. This requires a well-adapted consultation framework involving all partners: the government, civil society, NGOs, development partners, and the private sector. Significant progress has been made in creating such a participatory momentum and the government will continue: (i) building the capacities of social partners to engage in productive dialogue; (ii) maintaining a continuous dialogue through systematic consultations among social partners, including grassroots communities; (iii) promoting a climate of mutual confidence; and (iv) strengthening consultation and civil dialogue organs.

\subsubsection{Enforcing the rule of law and ensuring the security of goods and persons}

389. The government is well aware that the confidence of citizens and investors depends upon their perception of the effective existence of the rule of law in Cameroon, ensuring that the laws and regulations that govern society are applied fairly and evenhandedly. Therefore it plans to continue its efforts to enforce the rule of law and to ensure the protection of civil and political rights for all, equal access to justice, and legal security for persons, goods and investments.

390. With a view to consolidating the rule of law and the security of investments, in January 2002 the authorities launched a technical audit study of the Cameroonian legal system that should identify the problem areas explaining the poor performance of Cameroon's justice system and should recommend institutional measures to improve the quality of the judicial process in terms of turnaround time and appropriate enforcement of the rule of law. The study is expected to be completed in the course of 2003. One of its important outcomes would be to pave the way for the design of an action plan to introduce long-awaited reforms to the justice system, as its effective functioning is of vital importance for the development of private investment.

391. Significant progress has been made to create the Audit Court (Chambre des comptes) and the Constitutional Council (Conseil constitutionnel), two key institutions 
provided for in the Constitution of January 18, 1996. A draft law specifying the organization, composition, attributions, and operations of the Audit Court has already been prepared. It will be promulgated in 2003 and its President will be appointed in 2004. The legal status of its members is currently being drawn up and will be published in 2003. The law establishing the Constitutional Council is expected to be adopted and promulgated in 2003.

\subsubsection{Public resources management}

392. Expenditure Management. Significant progress has been made in implementing the action plan for the improvement of public expenditure management adopted in December 1998, especially in terms of the tracking of public expenditure. Key actions that have been taken include:

(i) Implementing the first phase of a deconcentration of the Integrated Civil Service Management System (SIGIPES);

(ii) Phasing in the Integrated Public Finance Management System (SIGEFI), including the adoption of a classification of budgetary expenditure by economic type (see box below).

(iii) Conducting audits in key areas, including: (i) organizational and technical audit of public control mechanisms and systems; (ii) audit of investment expenditure execution and control procedures; (iii) annual audits in the health, education, public works, agriculture, and livestock ministries; (iv) audit of the accounts and organizational and operational audit of SNH, the National Oil Company; (v) economic and tax audit of the forestry sector; and (vi) audit of procurement systems.

(iv) Appointing an independent observer in the logging license commissions and in procurement commissions. 


\section{Box 28. Budgetary Reform}

The budgetary reform undertaken since 1998 under the action plan for the improvement of public expenditure management offers the authorities a framework for accountability and good budgetary practices that sets out the conditions for successful implementation of government's governance and poverty reduction program.

\section{The reform addresses four priority areas:}

(1) Reform of the state budget nomenclature: The new budget nomenclature prepared with the assistance of the European Union and the IMF and World Bank offers three levels of classification: a functional classification, a classification of administrative expenditure units, and classification by economic nature of revenue and expenditure. It facilitates the execution of sound budgetary policies, strategy-based allocation of funds, accountability of financial managers, and effective monitoring of expenditure.

(2) Expenditure tracking by (i) securing fiduciary documents and streamlining their distribution; (ii) computerizing expenditure tracking through the DEPMI expenditure management system; and (iii) clarifying the roles of different actors in the chain of expenditure to avoid duplication and unnecessary control.

(3) Development of an information system, resulting in the implementation of DEPMI in all the 11 provincial sites for the monitoring of budgetary execution, followed by the interconnection between the 11 provincial sites and the central site to facilitate country-wide tracking of budgetary operations. Today, DEPMI produces management reports on budget execution and traces all budget operations from commitment to payment.

(4) Streamlining of budget execution procedures and controls, including (i) automation of price control through a computerized market price list at all levels of financial control; (ii) reducing the number of steps in the processing of files from 40 to four within five years; (iii) enhancing ex-ante and ex-post control to increase the efficiency of expenditure; and (v) preparing procedure manuals for users and financial managers to improve their understanding of the expenditure system.

Source: Budget Directorate/MINFI.

393. Mobilizing budgetary resources. Modernizing the tax system and strengthening the tax authorities (customs and taxes) are also essential to public finance stabilization and consolidation.

394. Important ongoing reforms have already resulted in a substantial productivity increase in the Tax Directorate. To sustain these gains, a new reform program, the "second generation reforms" (with a 2005 timeframe), has been developed to address the following priority areas:

- at the organizational level, the reorganization along functional lines of central services of the Tax Directorate, with the creation of a unit responsible for the management of large companies and of a training center;

- in terms of legislation, continued simplification of the tax system with the introduction of an income tax reform and continuation of studies for the creation of a value-based property tax;

- in terms of computerization, implementation of the computerization master plan, equipment of services and integration into the SIGIPES system;

- in terms of tax collection, enhancing tax recovery by posting qualified personnel to all tax offices; 
- in terms of training, participation in an initial course at ENAM, strengthening continuing education, and creating a Center for Retraining and Continuing Education;

- in terms of tax control, enhancing control programming and monitoring and making procedures less cumbersome and more efficient;

- in terms of assistance to taxpayers, the publication of a taxation procedures manual specifying the rights and obligations of taxpayers, creation of administrative structures to improve customer service to enterprises, including a specialized for large enterprises ,and authorized tax management centers for small taxpayers;

- enhancing administrative efficiency; (i) improving staff motivation with regular payment of allowances and a more enabling environment within the Tax Directorate; (ii) introducing new management and personnel evaluation methods, including management by objectives; (iii) systematic monitoring of taxpayers and the institution of a dialogue with economic operators; (iv) reorganization of Tax Centers along functional lines, making them one-stop tax offices that can service the full range of taxpayers' requirements (tax base assessment, control, collection, and disputes).

- in terms of communication, creation of a consultation framework for socioprofessional groups, simplification of tax forms, and creation of a website and of a telephone center.

395. The authorities have launched a vast program to restructure and modernize the Customs Administration with the dual objective of enhancing the mobilization of non-oil budgetary revenue and improving the competitiveness of the national economy and that of the CAEMC subregion in general. Its main objectives are to:

- increase revenues from international trade by modernizing customs administration, reducing tax exemptions, and combating physical and white collar fraud;

- establishing a customs tax system promoting competitiveness in the production and distribution of goods and services, with improved specialized taxation systems for different economic activities, including transit;

- reducing the duration of customs clearance for imports and exports at ports, airports, railways, and road transit points, particularly at the Douala port.

396. The government is aware that these are ambitious objectives. With the help of development partners, it has drawn up a detailed action plan that it is implementing under the supervision of an ad hoc committee. The action plan addresses eight priority areas:

- Simplifying customs procedures and streamlining control;

- Modernizing services and computer systems;

- Improving the monitoring and control of tax exemptions and suspensive arrangements;

- Designing and implementing a customs enforcement system through the strengthening of human resources and equipment;

- Designing and implementing a human resource incentive policy framework, improving working conditions and making staff more accountable;

- Reorganizing the Customs Directorate to better adjust its mission to changes in the domestic economic environment;

- Clarifying the respective roles of customs and its partners in external trade; 
- Improving transparency in the relation between customs and other public or private organizations involved in custom clearance procedures.

397. Specific measures in each of the priority areas have already been implemented. Preliminary results are encouraging in terms of enhanced revenues as well as the satisfaction of users of customs services.

398. More broadly, the revision of the decree of February 7, 1962 on the financial system of Cameroon began in 2001 with the assistance of the European Union and other development partners. It will lead in 2003 to the adoption of the Cameroon Public Finance Act that will update public finance legislation by integrating measures drawn up by the government in the context of past economic and financial programs as well as current reforms, in particular its introduction of multiyear programming of public expenditure. The new law should: (i) make accounts easier to read; (ii) improve the management of appropriations; (iii) make public finance procedures more efficient; and (iv) improve the accountability of public managers.

\subsubsection{Fighting corruption}

399. Fighting corruption is an essential component of the National Governance Program. During the consultations, the population ranked corruption as a key determinant of poverty, directly related to public management. The Priority Action Plan includes measures to curb excessive centralization of budget management, corruption among civil servants, the apparent impunity of public funds embezzlers, red tape in the state apparatus, and the paucity of information concerning public management made available to the ordinary citizens.

400. The creation of anticorruption units was among the first measures taken. Their membership includes members of the civil society and civil servants. They have been established in all ministries and in some public agencies. Their activities are coordinated by a National Anticorruption Observatory under the authority of the Prime Minister, Head of Government, who personally chairs the meetings of an ad hoc anticorruption committee. The government plans to make these units more effective with better financial resources and equipment.

401. In addition to the reforms specifically implemented under the Priority Action Plan to improve government anticorruption efforts, the government will carry out other reforms planned under the National Governance Program or included under the Poverty Reduction and Growth Facility (PRGF) arrangement. In particular, it is committed to improve reporting, including the production of periodic reports on budget execution in all ministries and parastatal organizations, and to enforce legal sanctions against public managers convicted of mismanagement or financial embezzlement.

\subsubsection{Procurement reform}

402. Reforming procurement is another priority aimed at improving public expenditure management. The reform objectives include: (i) integrating public procurement in the expenditure system; (ii) reducing corruption by enforcing ex-ante and ex-post control; (iii) regulating procurement through the Procurement Regulatory Agency (ARMP); (iv) making procurement officers more accountable; (v) overhauling laws and regulations relative to procurement, ensuring they are effectively enforced; (vi) speeding up the 
procurement process; and (vii) developing capacities and training all actors involved in procurement.

403. Important measures were taken as a result of this reform, including: (i) the publication of Decree No. 2000/155 of June 30, 2000 modifying and supplementing some of the provisions of Decree No. 95/101 of June 9, 1995 regulating procurement, and Decree No. 2000/156 of June 30, 2000 modifying and supplementing some of the provisions of Decree No. 95/102 of June 9, 1995 on the mandate, organization, and operation of procurement commissions; (ii) the dissemination of guidelines for the application of current procurement regulations; (iii) the creation of a new Procurement Regulatory Agency (ARMP) in February 2001; (iv) the legal obligation for procurement officers to prepare and update a procurement plan for the investment program; (v) the organization of workshops on procurement programming; (vi) the organization of several training sessions on the criteria used in the evaluation of bids and the use of qualification criteria of bidders; (vii) the appointment of a deputy chair of procurement commissions; (viii) the preparation of a booklet containing directives for bidders to public procurement and of a decree relative to procurement taxation, specifying tariffs and taxes to be borne by the government; (ix) institutionalized presence of independent observers in procurement commissions and recruitment of independent auditors to audit procurement contracts upon their completion; (x) the creation of a computerized records system at the ARMP; (xi) the harmonization of working methods and documents for independent observers; and (xii) developing a standard model for the invitation to bid. The reform should culminate in the adoption of a Procurement Code, which according to the reform's plan of action should become available by the end of the first half of 2003.

\subsubsection{Human resource management}

404. Progress has been made in two key areas: the delivery of social services and the civil service incentive framework, as illustrated in the education and health sectors. In the education sector for example, regulations have been introduced to gradually decentralize personnel management, improve the status of teachers, and involve civil society in the management of public schools (see paragraph 3.7.1).

405. A strategy has been adopted to ensure an efficient and decentralized management of teachers and other educational staff. It is based upon the following principles: (i) recruitment of teachers dictated by actual vacancies and budget availability; (ii) deconcentration of career management at the level of the "department" for preschool and primary school teachers, at the level of the province for secondary school teachers, while leaving recruitment of teachers and budget allocation at the central level, but on the basis of requirements expressed at the departmental or provincial level, and recruitment and wage payment at the ministerial level, as well as staff training on these new management procedures at all levels. Improving the status of teachers led to the progressive application, within the limits of available budgets, of the financial benefits provided for in Decree No. 2000/359 of December 5, 2000, granting a special status to the teaching profession in the Ministry of National Education.

406. A more participatory system of school management with representation of all stakeholders was introduced under Decree No. 2001/041 of February 19, 2001 on the organization of public schools and the attribution of school administration officials and its texts of application, notably the related by-laws and circulars concerning: (i) the functioning and management of an Education Development Solidarity Fund; (ii) the organization and 
functioning of public preschools and primary schools; (iii) the execution of public school budget and the functioning of School Councils, of the permanent Commission, and of a Council of Students' Delegates within public schools. The School Councils were effectively created and began operating during the 2001/2002 school year.

407. In the health sector, the government has issued Decree No. 2001/145 on the special status of public health workers, reflecting a consensus reached by the health worker unions and the authorities. Its provisions on the financial benefits for health workers took effect in 2002. Discussions on the introduction of a participatory management system were held in 2002 and should be concluded in 2003 with the publication of a legal text formalizing the consensus. Special attention has been paid to issues raised by the outsourcing of health work and to the collaboration between the Ministry and public or private health centers.

408. In addition to measures already taken to improve the delivery of education and health services, the government plans to carry out the following activities in 2003: (i) budget tracking to identify the sources of tax evasion and other problems contributing to the lack of effectiveness of public expenditure; and (ii) beneficiary assessment regarding the delivery of social services.

\subsubsection{Information to citizens}

409. The August 2000 Priority Action Plan includes specific actions to improve citizens' access to information on public management: (i) adopting, after conducting a diagnostic study, legislation giving citizens the right to information on public affairs and setting conditions for the free circulation of such information; and (ii) establishing appropriate institutional mechanisms to help each citizen obtain information on public affairs. The diagnostic study will be carried out during 2003 once the necessary funds have been secured. Pending the completion of this reform, steps have been taken to facilitate citizens' access to information on public management through the government information system.

410. In the area of grassroots development, the Priority Action Plan contains measures to facilitate the identification and execution of pilot community projects. An action plan for the implementation of this measure was adopted in November 2002. Its implementation is envisaged in 2003. By implementing this project, the government seeks to step up citizens' involvement in the daily management of public affairs at the level of local communities. It plans to extend the pilot experience throughout the country as decentralization is phased in. The pace of decentralization is expected to accelerate following the creation in the Ministry of Territorial Administration and Decentralization of a post of Minister delegate in charge of Decentralized Territorial Communities, with responsibility for implementing the reforms required to effectively complete the decentralization of the state. 


\subsection{THE CORE SCENARIO OF THE PRSP}

411. The PRSP sectoral policies were described in the foregoing chapter. The following sections analyze their estimated macroeconomic, budgetary, and social repercussions in five stages: (i) the quantitative impact of the sectoral strategies on the short and medium-term macroeconomic outlook; ii) simulation of their macroeconomic and financial effects; (iii) analysis of their effects on monetary poverty (incidence and distribution) and the other social dimensions; (iv) the medium-term public expenditure profile (medium-term budget framework - MTBF), cost, and financing; (v) analysis of the risks and alternative scenarios in the event of shocks or major deviations from the core scenario.

\subsubsection{Methodology}

412. The process: Continuing the participatory approach used in drafting the PRSP, the authorities have organized technical meetings with specialists in the different sectors in order to grasp specific aspects and to obtain growth estimates for each sector. With World Bank support, the education and health sectors have also listed their requirements and calculated the cost of meeting them. A study is also under way to achieve a more accurate estimate of the costs of the rural sector strategy. Their budgetary outlays take into account the availability of funds in the medium-term budget framework. All sectors have adopted the same iterative and participatory approach to refine their strategies and obtain a clearer overall picture.

413. The instrument: Before discussing the hypotheses and outcomes, it should be noted that the approach used to determine the growth profile is descriptive, not prescriptive. The growth rate is not set at the level required to achieve the poverty reduction objective, as it is, for instance, in a centralized planning system or a financial programming exercise. It is a product of the policies and programs implemented in the framework of the PRSP and of the behavior of economic agents. The growth profile is determined with the help of a macroeconometric model in which potential and sectoral outputs depend on three sets of factors: (i) the long-term determinants of productive capacity (human capital, infrastructure, direct investment flows); (ii) short and medium-term fluctuations in demand (relative prices, terms of trade); and (iii) sector-specific exogenous factors (voluntarist transformation policies in certain branches of activity, as in the case of the timber industry) or private sector investment programs (specifications of privatized firms: electricity, the railways, etc.).

414. The other key sources of information are: (i) the sectoral strategy papers describing the objectives, programs, and public and private sector efforts in priority areas, including education, health, and the rural sector; and (ii) studies of the sources of growth. 


\section{Box 29. Brief description of the macro-econometric model}

Methodology: An endogenous growth model was estimated to project short and medium-term growth by branch of activity. The determinants of growth comprise: (i) the fundamentals of medium and long-term production capacity, especially private physical capital driven by private investment, productive public capital (infrastructure, etc.), human capital driven by performance in education, health, and the job market and (ii) factors causing short-term fluctuations in demand (budgetary and monetary policies) or supply (natural or business cycle shocks), which are also included in the equations for determining sectoral growth rates. Dynamic econometric methods (error correction model) were used to estimate the equations of the Cameroonian model. The sectoral growth functions take the following generic form:

$Y=f($ Exogenous, Physical Capital, Human Capital, Current international economic situation)
Or Exogenous $=\{$ Sectoral strategies and new policies $\}$
Current international economic situation $=\{$ Prices, exchange rates, world growth $\}$

The other components of the macro framework are determined by accounting equations and standard financial programming principles. The Medium-Term Budget Framework breaks resources down into budget allocations by sector, according to exogenous codes reflecting government priorities.

\section{Sequence:}

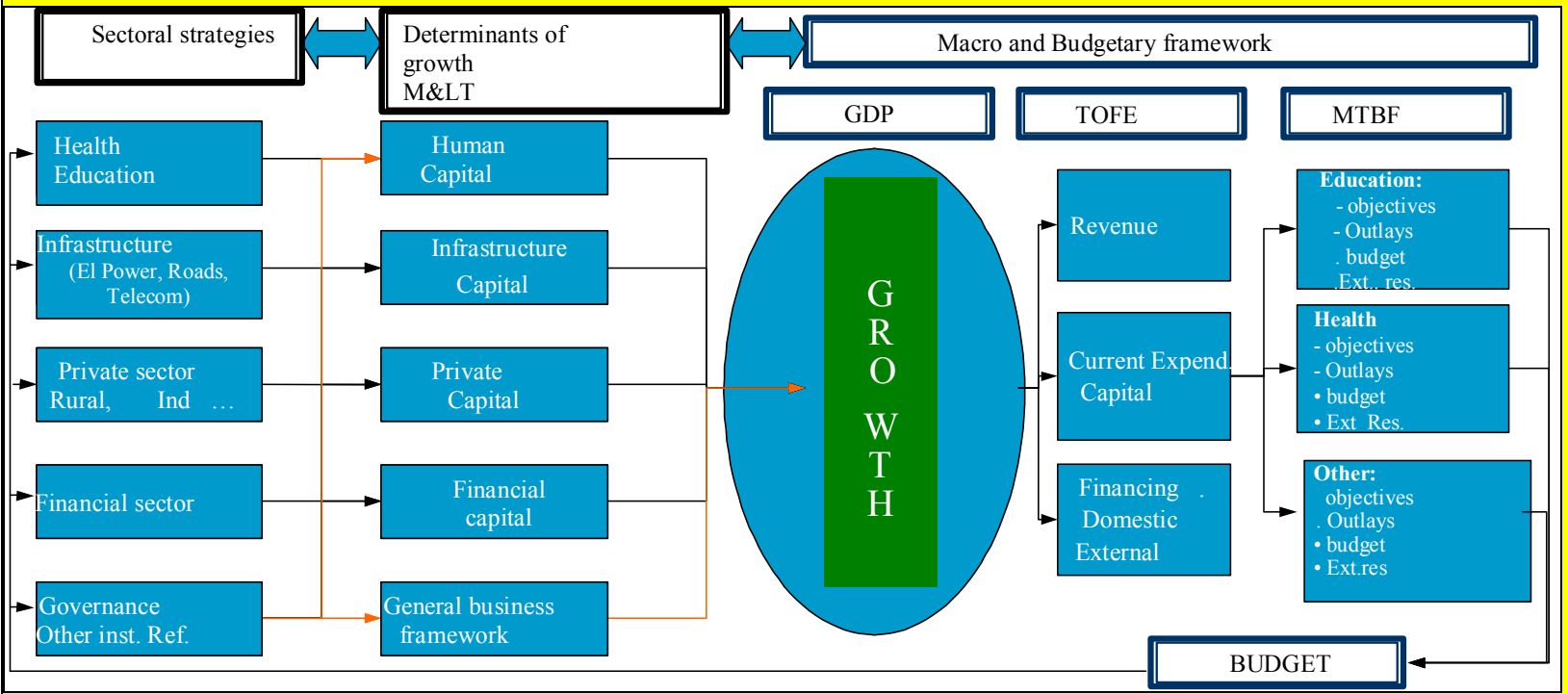

Outcomes: By way of illustration and to justify the choices made in the PRSP, the following simulation shows the effects on growth and poverty of allocating resources in the PRSP priority sectors: education, health, infrastructure, etc. The results indicate clearly that, if implemented effectively, the PRSP's mix of social and other policies would lead to annual growth rates of over six percent in the medium term.

\begin{tabular}{|c|c|c|c|c|}
\hline & \multicolumn{2}{|c|}{2007} & \multicolumn{2}{|c|}{2015} \\
\hline & Growth & Poverty & Growth & Poverty \\
\hline Extrapolation of current trends in the priority sectors $(\%)$ & 5.0 & 36.0 & 5.9 & 29.0 \\
\hline Growth entirely related to exactly the same Education (\%) & 5.3 & 35. & 6.4 & 27.0 \\
\hline outlays as those envisaged in the core Health (\%) & 5.4 & 35.0 & 6.6 & 26.8 \\
\hline scenario of the PRSP for: $\quad$ Infrastructure (\%) & 5.2 & 35.6 & 6.3 & 28.0 \\
\hline PRSP scenario combination & 6.0 & 37.1 & 7.1 & 25.2 \\
\hline
\end{tabular}

Source: MINFI/DP 


\subsubsection{The short-term outlook}

415. The short-term outlook is shaped by extrapolating from recent trends and reconciling the last year of the PRGF and the first year of implementation of the poverty reduction strategy. As in the previous two years, the macroeconomic context in 2003 will be characterized by a decline in the oil sector (-2.9 percent), accompanied by considerable uncertainty regarding oil prices until the Iraqi crisis has run its course. The power sector is also a source of concern, despite a projected increase in electricity output of 6.2 percent, offsetting the 4.6 percent decline in 2002.

416. Demand will continue to be driven by public expenditure of HIPC funds, with Cameroon expected to reach completion point and to implement the first year of the poverty reduction strategy. Use of the Chad-Cameroon pipeline should also boost subregional demand, given Chad's demographic weighting in the CAEMC, and thereby mitigate the negative impact of net exports on growth.

417. Based on the above hypotheses, growth in 2003 is projected at 4.5 percent, a slight decline compared to performance over the past five years, in which growth averaged 4.7 percent a year. Inflation, which picked up over the past two fiscal years as a result of increases in food crop prices, should slow to around 2.8 percent for 2003, thanks to improvements in the output and marketing of food crops. With revenue and expenditure totaling approximately 19 percent and 18 percent of GDP, respectively, there should be a fiscal surplus of approximately 1 percent of GDP in 2003.

\subsubsection{The sectoral strategies and medium-term growth potential}

418. Studies indicate that over the medium and long term the principal obstacles to sustainable growth in Cameroon are (i) human capital shortcomings, which translate into low labor productivity; (ii) insufficient infrastructure, which means high costs and weak factor competitiveness and (iii) the dearth of effective investment. This impression is confirmed by the findings of the participatory consultations, which pointed to infrastructure (roads, access to drinking water, etc.) and to the education and health sectors as the priorities of the Cameroonian population. These three pillars - human capital, infrastructure, and physical and financial productive capital - are built into the model as the determinants of medium-term growth.

419. Human capital. The work force comprises approximately 50 percent of the population. The economically active population has had, on average, three years of primary education. ${ }^{13}$ While this average is among the higher levels found in Central and West African countries, it is weak for growth based on human, rather than natural, resources. The Government intends to promote the development of human resources as the cornerstone of vigorous and more equitable growth. Indeed, apart from the direct effect of general government on noncommercial GDP, expenditure on education has a long-term indirect impact on productive capacity by generating an increase in the stock and productivity of human capital. It is estimated that expenditure in the education sector will increase the human capital stock by approximately 10 percent over the next ten years. That will raise GDP growth by one percent over the period.

\footnotetext{
${ }^{13}$ Sacerdoti and Tang, (1998), "The impact of human capital on growth : evidence from West Africa”, IMF WP/98/62
} 


\begin{tabular}{|c|c|c|c|}
\hline & Country & Methods & $\begin{array}{c}\text { Impact on } \\
\text { growth of GDP }\end{array}$ \\
\hline Arnd and Lewis (2000) & South Africa & CGE & -0.8 to -1 \\
\hline Bonnel (2000) & 47-86 countries & "cross section" & -0.7 \\
\hline $\begin{array}{l}\text { Kambou, Devarajan and } \\
\text { Over (1991) }\end{array}$ & Cameroon & CGE & -0.5 to -1.2 \\
\hline Over (1992) & 30 & $\begin{array}{l}\text { Economic \& } \\
\text { demographic modeling }\end{array}$ & -0.3 to -0.6 \\
\hline Sackey and Raperla & $\begin{array}{l}\text { Botswana, Lesotho, } \\
\text { Namibia, Swaziland }\end{array}$ & $\begin{array}{l}\text { Economic \& } \\
\text { demographic modeling }\end{array}$ & -1 to -1.5 \\
\hline
\end{tabular}

420. Health, like education, is both a social objective and a factor stimulating growth by enhancing human capital. It is estimated that 71 percent of deaths in Africa are caused by diseases that could be eradicated. The incidence of AIDS signifies an enormous economic cost. That of malaria shortens life expectancy and reduces GDP by between 1 and 1.5 percentage points. The estimates underscore that a 5 percent increase in expenditure on health is associated with a one-fifth of one percent net increase in GDP growth over the medium term. ${ }^{14}$.

421. Infrastructure affects transaction and production costs and therefore the overall competitiveness of the economy. Road infrastructure has a significant impact on agriculture. Electricity is a major factor in industry, and transportation and telecommunication facilities are key factors for the services sector. As discussed in Chapter 3, the strategy of boosting and diversifying growth relies on the development of human capital, accompanied by a comparable increase in the volume and quality of physical capital, particularly infrastructure. Indeed, the estimations show that a 5 percent increase in the average stock of infrastructure translates into an increase of approximately 1.5 percent in medium and long-term productive capacity.

422. Private investment and foreign investment: Improvements in infrastructure, human resources, and the business environment help to raise the rate of private investment and the cost-effectiveness of total investment. In 2001, investment was approximately 18 percent of GDP, with private sector investment accounting for 15.5 percent and the public sector 2.5 percent. The apparent effectiveness of the investment averaged around 25 percent (an incremental capital-output ratio of 4). Such a ratio is not enough to sustain increased diversification of the economy. Even with enhanced effectiveness, the investment ratio needs to be in the order of 25 percent of GDP to sustain average annual growth of 6 to 7 percent. The experience of a number of countries that have achieved economic take-off and managed to cruise at 6-8 percent annual growth rates suggests that this kind of simultaneous increase in

\footnotetext{
${ }^{14}$ The impact of health on growth is often measured by variables such as the population/number of doctors ratio. The budget is more widely distributed in Cameroon than medical specialists, who are concentrated in Yaoundé and Douala. Health expenditure would therefore appear to be a more appropriate explanatory variable of the impact of health.
} 
both the volume and effectiveness of investment requires a major inflow of foreign investment. $^{15}$

423. The foreign direct investment/GDP ratio, which is still weak considering per capital income and the country's potential, declined from 1.36 in 1991-1994 to 1.17 in 1995-1998, while the same ratio tripled for the sub-Saharan African countries as a whole. ${ }^{16}$ It is estimated that current efforts to promote private sector development and good governance, and to complete the privatization program in Cameroon will take this ratio up to 1.5 percent by 2007 and to approximately 2 percent of GDP by 2010 to sustain growth.

\subsubsection{The medium-term growth outlook by branch of activity}

424. Growth in recent years has relied partly on the oil sector. Its contribution will, however, decline over the next 10 years. The government's efforts to stimulate growth should therefore be directed to strengthening competitive advantages in other branches of activity, including industrial activities, so that they can replace oil and generate more diversified and more equitably distributed growth. These policies and programs have begun to translate into quantified medium and long-term sectoral growth outlooks. The following sections assess the implications of these policies for growth projections in the main branches of activity.

425. Food agriculture: The rural development strategy aims to increase food output by 10 percent per year, thereby raising both supply and the share of food agriculture in the growth of GDP and of exports. Demand from oil-exporting neighboring countries (Chad, Equatorial Guinea, Nigeria, Angola) combined with strong growth of the urban population (4.8 to 5 percent a year) will ensure that there is a market for food products. On the supply side, policies designed to enhance factor productivity and specific programs for increasing yields and expanding land used for farming will make it possible to meet that increased demand. Thus food agriculture is expected to grow nearly 4 percent a year in the medium term, up a good two points over annual growth for the past ten years.

426. Traditional export crops will grow at a moderate pace. The (nonoil) export price index is expected to grow 6 percent on average. Studies of the sources of growth indicate that other products could emerge and take off following implementation of specifically targeted promotional policies, as happened with pepper, for instance. These trends thus point to an increase in the rate of growth from 2.8 percent in 2003 to 4.2 percent in 2015.

427. Fishing and livestock farming: Improvements expected in livestock farming and fisheries could also increase their shares in growth, with rates averaging 4 percent and 2.5 percent, respectively, over the projection period.

\footnotetext{
${ }^{15}$ According to Borensztein et al (1998) and Wacziarg (1998), a 1 percent increase in foreign direct investment produces an increase in domestic investment of between 0.5 and 1.3 percent, depending on the country concerned, as well as a 0.3 to 0.8 percent increase in the rate of growth of GDP per capita.

${ }^{16}$ M. Pigato, 2000, «Foreign Direct Investment in Africa : Old Tales and New Evidence », Africa Region Working Paper Series, No 8, World Bank, November 2000.
} 
428. Forestry: The forestry sector should regain its former dynamism. In response to the law restricting exports of unbarked logs, several operators have imported obsolete material to start initial processing of the timber. With the increase in forestry license fees and the new system for allocating forestry development units, only more advanced processing will be profitable and, by the same token, encourage exploitation of so far neglected species. Moreover, assignment of all the forestry units will reduce the amount of illegal felling and expand the formal logging industry's output. British technical cooperation experts reckon that the sector could grow 8 percent per year. Taking into account adjustment lags and reticence on the part of operators who would like to secure a profit on the material they already have, the framework model assumes growth in output from 2.07 million cubic meters in 2002 to approximately 3 million in 2006 and 3.5 million in 2015, that is to say, an annual average growth of 4 percent over the projection period.

429. Certain branches of manufacturing, such as wood processing and agroindustry have comparative advantages ${ }^{17}$. These advantages should lower factor costs and increase productivity once policies to develop human resources and infrastructure have been implemented. Furthermore, electricity output will eventually increase substantially (at an annual average rate of over 6 percent) thanks to investments being studied or expected from privatization. These factors will revive manufacturing industry, especially metalworking industries, textiles, and the cement industry. The rate of growth of manufacturing could thus reach 8-10 percent by 2015, an average gain of more than three points over the period, compared to the past five years.

430. Construction and Public Works (BTP) would benefit from budget allocations in the priority sectors (health, education, the rural sector) rural) for the construction and rehabilitation of basic infrastructure, in addition to the impact derived from a housing policy. Consequently, this sector is projected to enjoy accelerated annual growth of approximately 7.5 percent over this period.

\footnotetext{
${ }^{17}$ A. H. G. Nkama, «Etude sur les Sources de la Croissance pour la Réduction de la Pauvreté au Cameroun: Compétitivité des filières industrielles» [Study on the Sources of Growth for Reducing Poverty in Cameroon: Competitiveness of branches of industry] June, 2002.
} 


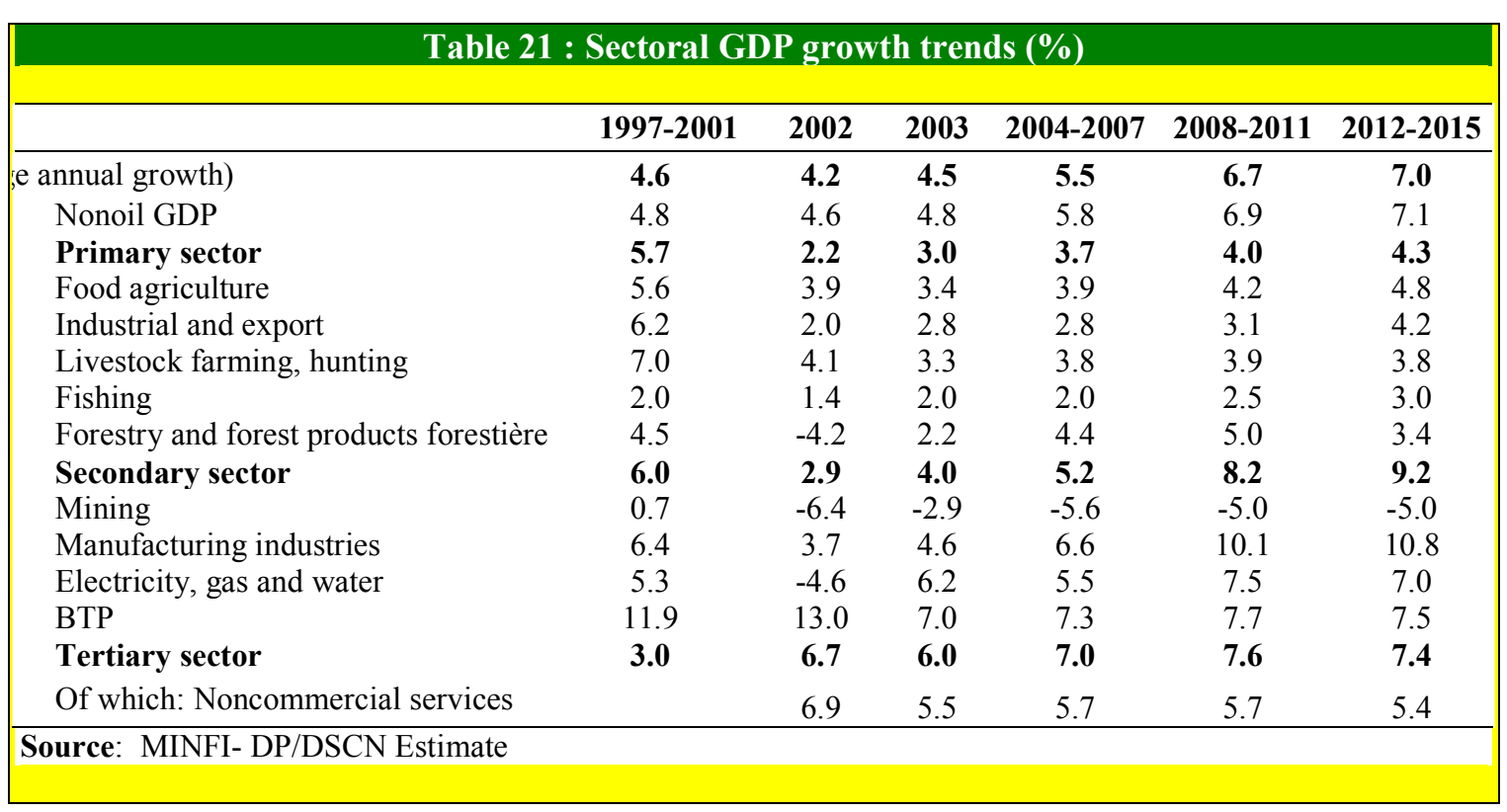

431. Commercial services should continue to expand in tandem with trends in the primary and secondary sectors (trade and transportation). This expansion will also be fed by noncommercial general government services in the context of public expenditure related to implementation of the strategy. Likewise, communication technology-related activities (mobile telephony, the Internet) will continue to enjoy the remarkable growth rates of the past few years. Moreover, as the financial market gets off the ground (the Douala securities exchange, and Treasury bond issues as of 2004), the financial sector will be transformed and the additional expansion of financial services will constitute another growth factor. Finally, plans to develop the tourist industry, if actually implemented, could increase this subsector's contribution to national output. It is projected to average 7.5 annual growth over the period.

\subsubsection{The medium-term macroeconomic and financial framework}

\subsubsection{Gross national product: a breakdown}

432. Based on the sectoral developments outlined above, GDP should achieve sustained medium-term growth. From 4.5 percent in 2003, the growth rate should reach 6 percent in 2007 and remain steady at around 7 percent between 2011 and 2015, in other words annual growth would average 6 percent, a point and a half net improvement over growth in 19992002. As a result, income per capita would enjoy a net increase of approximately 3 percentage points a year over the projection period. Thanks to dynamic growth in the food output sector, the primary sector as a whole would grow by an additional two percentage points a year between 2002 and 2010, the non oil secondary sector by five points, and the tertiary sector by almost one point.

433. On the demand side, the trade deficit, which widened in 2002, turns into a surplus as of 2003. The current account balance is expected to continue to show a deficit with the decline of oil export revenue but to improve gradually thereafter as the nonoil sector takes off and eventually to show a surplus between 2011 and 2015. Despite declining oil output, exports are in fact expected to increase as a percentage of GDP thanks to food farming and manufacturing output. Imports, which have recently surged with the construction of the Chad-Cameroon 
pipeline, are projected to grow at more moderate rates over the projection period, even though capital goods imports will continue to expand as a result of economic diversification.

\begin{tabular}{|c|c|c|c|c|c|c|c|c|c|c|c|c|c|c|c|c|}
\hline & \multicolumn{3}{|c|}{ Estimates } & \multicolumn{13}{|c|}{ Projections } \\
\hline & 2000 & 2001 & 2002 & 2003 & 2004 & 2005 & 2006 & 2007 & 2008 & 2009 & 2010 & 2011 & 2012 & 2013 & 2014 & 2015 \\
\hline Domestic demand & 96.8 & 99.1 & 101.5 & 101.4 & 102.1 & 102.7 & 102.7 & 102.0 & 101.4 & 100.8 & 100.2 & 99.8 & 99.7 & 99.7 & 99.7 & 99.5 \\
\hline Consumption & 79.6 & 81.0 & 83.4 & 83.1 & 83.7 & 83.8 & 83.4 & 82.3 & 81.2 & 80.1 & 79.0 & 78.1 & 77.6 & 77.1 & 76.7 & 76.0 \\
\hline Private sector & 68.9 & 69.7 & 71.7 & 71.2 & 71.7 & 71.6 & 71.1 & 70.0 & 69.0 & 68.0 & 67.0 & 66.3 & 65.9 & 65.6 & 65.4 & 64.9 \\
\hline General government & 10.7 & 11.3 & 11.8 & 11.9 & 12.0 & 12.1 & 12.2 & 12.2 & 12.2 & 12.1 & 12.0 & 11.8 & 11.6 & 11.5 & 11.3 & 11.1 \\
\hline Investment & 17.1 & 18.1 & 18.1 & 18.3 & 18.5 & 18.9 & 19.3 & 19.7 & 20.3 & 20.7 & 21.2 & 21.7 & 22.2 & 22.7 & 23.0 & 23.4 \\
\hline GFCF & 17.1 & 18.1 & 18.1 & 18.3 & 18.5 & 18.9 & 19.3 & 19.7 & 20.3 & 20.7 & 21.2 & 21.7 & 22.2 & 22.7 & 23.0 & 23.4 \\
\hline Private sector & 14.2 & 15.2 & 15.3 & 15.2 & 15.4 & 15.8 & 16.2 & 16.6 & 17.0 & 17.3 & 17.7 & 18.2 & 18.6 & 19.0 & 19.5 & 19.9 \\
\hline General government & 2.9 & 2.9 & 2.7 & 3.1 & 3.0 & 3.1 & 3.1 & 3.2 & 3.3 & 3.4 & 3.5 & 3.5 & 3.6 & 3.6 & 3.5 & 3.5 \\
\hline Changes in inventory & 0 & 0 & 0 & 0 & 0 & 0 & 0 & 0 & 0 & 0 & 0 & 0 & 0 & 0 & 0 & 0 \\
\hline Net exports & 3.2 & 0.9 & -1.5 & -1.4 & -2.1 & -2.7 & -2.7 & -2.0 & -1.4 & -0.8 & -0.2 & 0.2 & 0.3 & 0.3 & 0.3 & 0.5 \\
\hline Exports of goods \& serv. & 31.3 & 29.5 & 28.0 & 27.5 & 26.1 & 25.1 & 24.6 & 24.7 & 24.9 & 25.1 & 25.4 & 25.8 & 25.8 & 25.9 & 26.1 & 26.5 \\
\hline Imports of G \& S & 28.0 & 28.6 & 29.5 & 28.9 & 28.3 & 27.8 & 27.3 & 26.7 & 26.3 & 25.9 & 25.6 & 25.5 & 25.5 & 25.6 & 25.8 & 25.9 \\
\hline GDP at market prices & 100,0 & 100,0 & 100,0 & 100,0 & 100,0 & 100,0 & 100,0 & 100,0 & 100,0 & 100,0 & 100,0 & 100,0 & 100,0 & 100,0 & 100,0 & 100,0 \\
\hline $\begin{array}{r}\text { Sources: IMF (1995/199 } \\
\text { MINFI/DP (Pr }\end{array}$ & 1) & & & & & & & & & & & & & & & \\
\hline
\end{tabular}

434. Growth is projected to be sustained by investment, with the investment ratio averaging 23.4 percent of GDP by 2015, a 5-point gain over 2002, reflecting above all dynamic private investment supported by government and foreign investment efforts. This upturn is based on a hike in private savings, following moderate but sustained growth of private consumption (approximately 5.7 percent). Consumption per capita is thus expected to achieve a net 3 percent annual gain over the period, which indicates an improvement in the standard of living of the population.

\subsubsection{Money}

435. In line with community options, the projections assume gradual elimination of drawings $^{18}$ on the BEAC (at a rate of $1 / 10$ per annum for loans to the Treasury of over CFAF 225 billion and CFAF 22 billion in the case of consolidated loans) and on the banking system as a whole (at the rate of CFAF 10.8 billion per annum). This results in a reduction in net credit to the government and a comparable increase in credit to the private sector, which is projected to increases its share of GDP by 4.6 points between 2003 and 2015. By injecting liquidity into the economy, repayment of the domestic debt makes it possible to sustain consumption or strengthen private saving, to finance private investment or, possibly, government requirements by purchasing Treasury bonds, as is envisaged as of 2004 . It is noticeable, however, that, despite a three-point gain over the period, the ratio of money supply to GDP remains low in Cameroon compared to countries with comparable per capita income. (Cf. Table of Principal Indicators).

\footnotetext{
${ }^{18}$ Source: BEAC.
} 


\subsubsection{The balance of payments and external indebtedness}

436. The trade balance improves in the projections, despite a decline in oil exports, because of an upswing in the nonoil sector. Reduction of the burden of interest on the foreign debt will lead to an improvement in the current account and overall balances. Consequently, gross foreign assets are consolidated at approximately 30 percent of sight liabilities of the monetary system, which exceeds the statutory ceiling of 20 percent. The projections indicate that, with the scheduled debt relief, the external debt remains sustainable. There is even a net improvement in sustainability ratios. In particular, debt servicing diminishes as a percentage of exports.

\subsubsection{Public finance}

437. Despite major government efforts in support of the strategy, public expenditure is projected to remain stable as a percentage of GDP (approximately 17 percent over the whole period). That is partly thanks to the reduction in debt servicing outlays, which has made it possible to cover the bulk of priority expenditures. At the same time, tax and customs reforms currently under way, the expansion of the tax base by, inter alia, trimming exemptions and enforcing payment of special taxes (in the forestry, livestock farming, fishing, and mining sectors), and the income tax reform should enhance nonoil revenue as a percentage of nonoil GDP. As a result, the fiscal deficit should narrow and financing requirements would remain moderate, compared with the size of the growth effort.

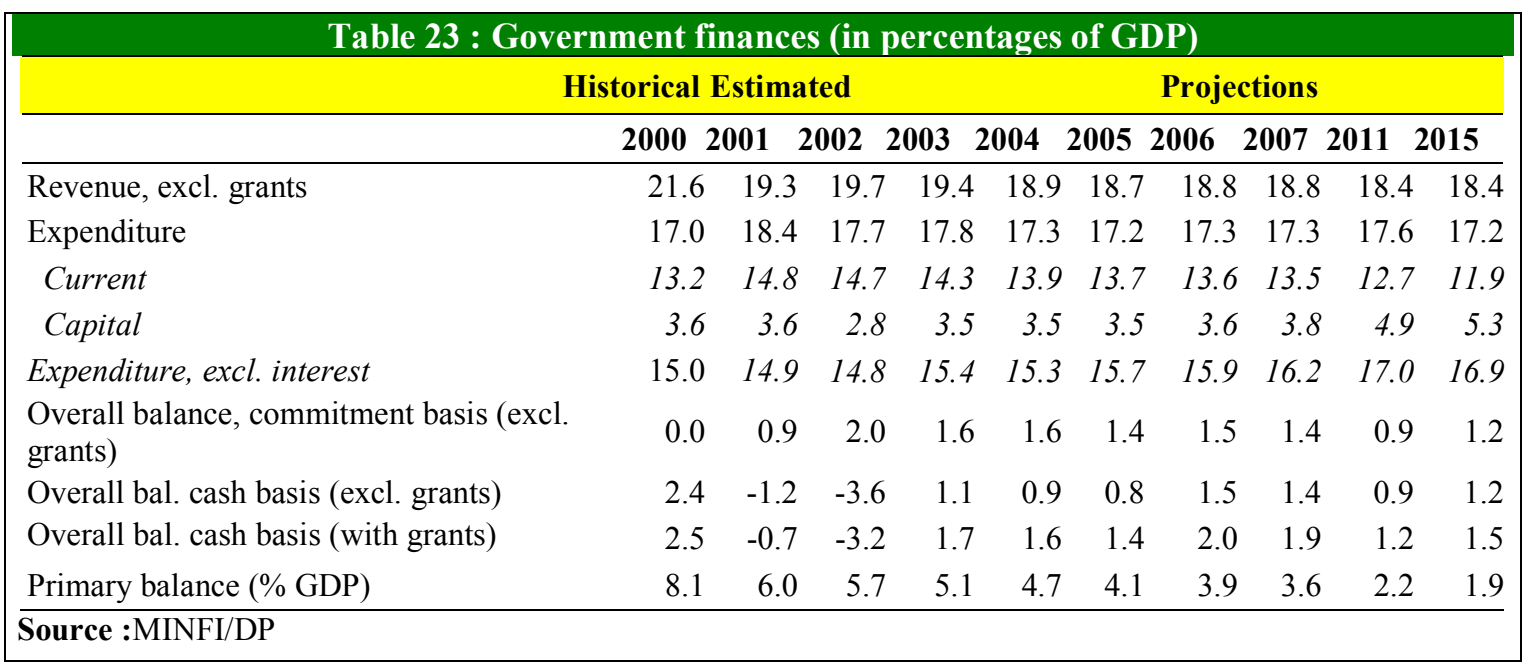

438. Revenue: In order to maintain macroeconomic stability, the government is committed to intensifying efforts to offset the decline in oil revenue by increasing nonoil revenue. That will enable it to meet the targets under the PRGF program, particularly the goal of increasing nonoil revenue to over 16 percent of nonoil GDP (16.4 percent in 2003). This upward trend reflects growth in the nonoil GDP and expectations with regard to the performance of the financial authorities.

439. Public expenditure - especially, wages, outlays for goods and services, and capital expenditure associated with implementation of the sectoral strategies for health, education, and infrastructure - is projected to grow over the initial years of the period. Wages, transfers, 
and subsidies are likely to increase by half a percentage point of GDP in 2007 and two and a half points by 2015 . This trend is possible without exceeding budgetary expenditure limits thanks to the reduction in debt servicing.

440. Financing: the overall balance (on a commitment basis, excluding grants) should improve, stabilizing at approximately 1.5 percent of GDP. That balance indicates that the strategy is indeed sustainable. Financing requirements appear moderate over the whole period, given the size of the growth effort. In fact, the resources expected at the conclusion of the Paris Club negotiations and as a result of additional debt forgiveness by France under the C2D initiative should cover most of the financing requirements. The authorities also intend to borrow from the population by issuing Treasury bonds to finance the residual requirements, as of 2004.

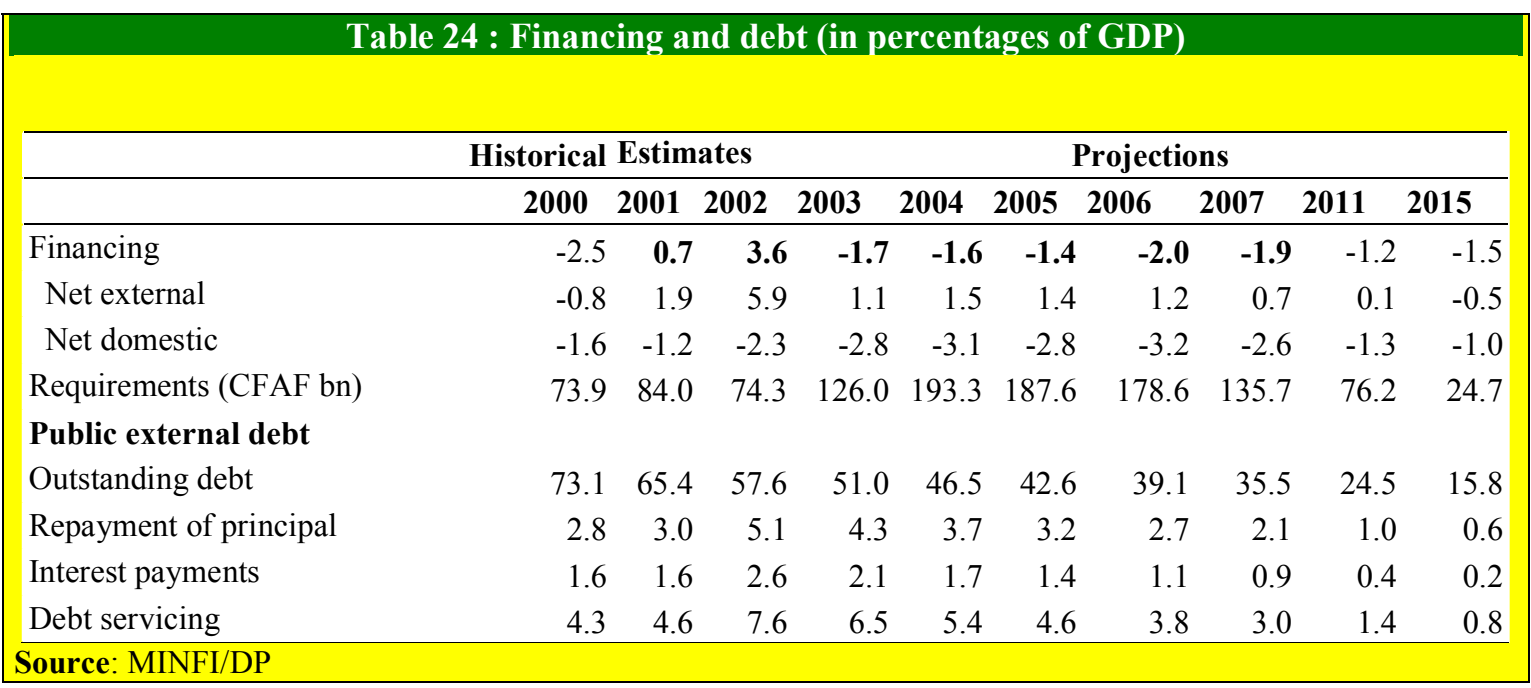

\subsubsection{The medium-term budget framework (MTBF)}

441. The MTBF helps to ensure financial compatibility between the sectoral strategies and the National Budget. It makes it possible to quantify the budgetary implications of the sectoral strategies and to reconcile the financing requirements of the strategies with the resources generated by growth (own funds) or derived from external assistance. The MTBF thus ensures consistency between budget estimates in which expenditure is classified functionally (by sector or ministry) and the Government Funds Flow Account ("TOFE") estimates, for which they are aggregated and projected according to economic category (current or capital). Finally, the MTBF guarantees consistency between the level of operating expenses and capital outlays.

442. Apart from these functions, the MTBF is also a tool for targeting budgetary policy. Indeed it is by using the budgetary allocation codes that the government establishes trade-offs in the assignment of resources to priority and other sectors. In this way it can stimulate certain sectors. These funds have a direct impact on achievement of sector goals and an indirect effect on growth.

443. In line with the government's option, the MTBF of the PRSP reflects a strengthening of priority sectors, especially the social sectors, during the first five years of the strategy (20032007). That will make it possible to recover lost ground, above all in education, health, and infrastructure. However, as the minimum sectoral targets are met, the emphasis will shift to 
expenditure supporting the productive sector, especially infrastructure and support for productive and export-oriented activities.

444. Education: the education sector strategy envisages budgetary support that will take the share of education in primary expenditure from 17 percent in 1995-2000 to 25 percent in 2001-2010. Capital expenditure is projected to grow faster than operating expenditure because of catch-up needs and due to special efforts to boost technical education and vocational training.

445. Health: The government's efforts in this sector focus on strengthening basic health care coverage and stemming the spread of endemic diseases such as AIDS. Implementation of the sectoral strategy will involve a major increase in budgetary outlays that will take the health sector's share of primary expenditure from 5 percent in 2000 to 10 percent in 2008 and toward the goal of 2 percent of GDP in 2015. According to the strategy, this growth will be spread uniformly among salaries (incentives, training), other operating expenses (medicine, maintenance, etc.), and capital outlays.

446. Productive infrastructure emerges in the participatory consultations with the population as one of its priorities. Based on the MTBF, the growth of budget expenditure in this field will increase its share from 1.5 percent in 2003 to approximately 3 percent in 2015. Thanks to structural reforms, including privatizations, this effort will be on top of growing private sector involvement, especially in electric power, water, and telecommunications.

447. Most of the poor in Cameroon are to be found in rural areas. Poverty reduction therefore implies developing those areas. Efforts to improve both infrastructure and crop yields are to be stepped up. As a result, the share of this sector in primary expenditure is projected to increase from 4 percent over the past five years to 7 percent in the projection period. This increase looks modest, given the importance of the sector, but it refers only to the budgetary cost of supplementary programs, not to expenditure on education, health, and infrastructure in rural areas. 


\section{Box 30. Growth elasticity of poverty in Africa}

\section{The issue}

The first of the eight principal millennium development goals is to halve, by 2015 , the percentage of people living below the poverty line (US\$1 per person per day), compared to 1990 . That raises a key question about the level of economic growth required for each country, given the structure of its economy.

\section{The methodology}

One way to address this issue is to resort to a simple model of the dynamics of monetary poverty. Its basic tenet is that changes in monetary poverty depend on both the average rate of growth ("growth effect") and the behavior of monetary inequality ("distribution effects"). The stronger and more sustained the rate of growth, the greater the impact on the poverty rate. However, the greater the inequalities, the weaker the impact of growth on poverty reduction. This can be expressed as a simple equation breaking down the behavior of the poverty rate:

Growth $($ poverty rate $)=$ el $1 *$ growth $($ income per capita) + el $2 *$ growth (inequality index), where ell and el2 represent the growth rate elasticity and the inequality index elasticity of the poverty rate, respectively.1/

\section{The outcomes and implications for Cameroon.}

This equation has been estimated for a great many African countries. The numbers show that in order to halve poverty by 2015 , for Africa as a whole, based on 1990s conditions, average annual growth would have to be 6.79 percent. For Cameroon, the figures indicate an income per capita elasticity of the poverty rate of approximately 1.18. This means that, assuming no change in inequality, a one percent increase in per capita income would translate into a 1.18 percent reduction in the poverty rate. These outcomes are within the range of those obtained on the basis of the ECAM surveys, taking the sectoral distribution of growth into account.

The upshot of these analyses is that Cameroon will indeed need to grow by a sustained 6-7 percent a year if it is to attain the first of the development goals of the millennium. The macroeconomic framework projections for Cameroon's PRSP outline the sectoral and temporal parameters for growth of this magnitude.

\section{Table 26 : Relation between rate of growth and the poverty rates in some African countries}

\begin{tabular}{lccccc}
\hline Country or Region & $\begin{array}{c}\text { Average } \\
\text { consumption per } \\
\text { inhabitant (PPA } \\
1985)\end{array}$ & $\begin{array}{c}\text { Poverty } \\
\text { index in } \\
\%\end{array}$ & $\begin{array}{c}\text { Gini } \\
\text { coefficient } \\
\%\end{array}$ & $\begin{array}{c}\text { Growth } \\
\text { elasticity of } \\
\text { poverty }\end{array}$ & $\begin{array}{c}\text { Required rate } \\
\text { of growth } \\
\%\end{array}$ \\
\hline Cameroon & $\mathbf{1 0 6}$ & $\mathbf{5 0 . 2}$ & $\mathbf{4 3}$ & $\mathbf{- 1 . 1 8}$ & $\mathbf{5 . 5 8}$ \\
Côte d'Ivoire & 56 & 29.49 & 37 & -1.06 & 5.90 \\
Senegal & 71 & 51.16 & 54 & -1.08 & 6.30 \\
Gabon & 127 & & 43 & -1.01 & 6.66 \\
Kenya & 59 & 49.37 & 58 & -0.58 & 9.6 \\
Africa & 76 & 43,5 & 43 & $-0,95$ & 6,79 \\
\hline
\end{tabular}

Source: Economic Report on Africa, 1999 by the U.N. Economic Commission for Africa - ECA 1999

1 / Note: The exact equation used is: $g(p 0)=\varepsilon \cdot(1-\eta) \cdot g(y)+\omega \cdot g(q)$; where $\varepsilon$ is the income per capita elasticity of the poverty rate; $\eta$ the income per capita elasticity of the poverty line; and $\omega$ the inequality coefficient $q$ elasticity of the poverty rate. 


\begin{tabular}{|c|c|c|c|c|}
\hline Key indicators: & $1996-2001$ & $2002-2007$ & $2008-2010$ & $2011-2015$ \\
\hline $\begin{array}{l}\text { National income (average annual growth) } \\
\text { Core scenario } \\
\text { Extrapolation of recent trends (1996-2002) }\end{array}$ & $\begin{array}{l}4.7 \\
4.7\end{array}$ & $\begin{array}{l}5.1 \\
4.7\end{array}$ & $\begin{array}{l}6.7 \\
4.5\end{array}$ & $\begin{array}{l}7.0 \\
4.5\end{array}$ \\
\hline $\begin{array}{l}\text { Per capita income (average annual growth) } \\
\text { Core scenario } \\
\text { Extrapolation of recent trends } \\
\end{array}$ & $\begin{array}{l}1.9 \\
1.9 \\
\end{array}$ & $\begin{array}{l}2.3 \\
1.9 \\
\end{array}$ & $\begin{array}{l}3.9 \\
1.7 \\
\end{array}$ & $\begin{array}{l}4.2 \\
1.7 \\
\end{array}$ \\
\hline $\begin{array}{l}\text { End-of-period poverty rate, elasticity } \mathbf{- 1 , 0 5} \\
\text { Core scenario } \\
\text { Extrapolation of recent trends }\end{array}$ & $\begin{array}{l}40.2 \\
40.2 \\
\end{array}$ & $\begin{array}{l}35.3 \\
36.2 \\
\end{array}$ & $\begin{array}{l}28.1 \\
33.0 \\
\end{array}$ & $\begin{array}{l}21.9 \\
30.0 \\
\end{array}$ \\
\hline $\begin{array}{l}\text { End-of-period poverty rate, elasticity }=\mathbf{- 0}, \\
\text { Core scenario } \\
\text { Alternative extrapolation of recent trends } \\
\end{array}$ & $\begin{array}{l}40.2 \\
40.2 \\
\end{array}$ & $\begin{array}{l}37.0 \\
37.5 \\
\end{array}$ & $\begin{array}{l}31.9 \\
35.3 \\
\end{array}$ & $\begin{array}{l}27.2 \\
33.2 \\
\end{array}$ \\
\hline $\begin{array}{l}\text { Source: MINFI- DP/DSCN estimate } \\
\text { (1) Elasticity for Cameroon is estimated at } \\
\text { for African countries, with only weak transm }\end{array}$ & $\begin{array}{l}\text { taking distrib } \\
\text { between grol }\end{array}$ & $\begin{array}{l}\text { on effects int } \\
\text { and poverty }\end{array}$ & $\begin{array}{l}\text { account; the c } \\
\text { duction. }\end{array}$ & erage is 0.7 \\
\hline
\end{tabular}

449. The projections indicate that with growth averaging six percent per annum over the period, the targeted poverty rate of 20 to 25 percent by 2015 would be achieved. The figures also indicate that with the growth path extrapolated from recent trends, and elasticity in the 0.7 to 1.05 range, the poverty reduction target would not be met. With a weaker response of poverty to growth (elasticity of -0.7 ), however, the millennium goal would not be achieved.

450. A more refined analysis, taking into account the sectoral distribution of growth, confirms the above findings. Given the different redistributive effects, the impact on poverty reduction varies according to the sector the growth comes from. The contribution of each branch of activity to poverty reduction will therefore depend on the extent to which growth impinges on poverty ("transmission") and on the weight of the sector in the total working population. Thus, one percentage point of growth in the rural sector could contribute more to poverty reduction than one point of growth in the oil sector. The findings of the ECAM household survey have made it possible to infer the incidence of poverty from the rate of growth of each sector. The overall poverty rate is thus the average of the sectoral rates weighted by each sector's share of the working population.

451. As mentioned earlier, the poverty rate projection taking sectoral distribution into account is in the range of 20 to 25 percent by 2015 . However, it varies considerably from one branch of activity to another. For example, in households in which the head of household works in the primary sector, the poverty rate is projected to decline by approximately 22 points from its 2001 level. That decline is only three points in households where the head of household works in the secondary sector, despite stronger growth in the secondary sector than in the primary sector. This is because of transmission channels between growth and poverty are less effective in the secondary sector. 


\begin{tabular}{|c|c|c|c|c|c|c|}
\hline & & 1996 & 2001 & 2007 & 2011 & 2015 \\
\hline \multirow[t]{2}{*}{ Overall } & Income (av. annual gr) & 5.0 & 4.8 & 6.0 & 7.0 & 7.1 \\
\hline & Poverty ( $\%$ pop, weighted average) & 53.3 & 40.2 & 37.1 & 31.7 & 25.2 \\
\hline \multirow{2}{*}{$\begin{array}{l}\text { Primary sector } \\
\text { (elasticity : }-1,2 \text { ) }\end{array}$} & Income (av. annual gr) & 0.1 & 0.7 & 3.8 & 4.0 & 4.6 \\
\hline & Poverty (\% pop.) & 62.7 & 54.1 & 49.7 & 43.2 & 32.5 \\
\hline \multirow{2}{*}{$\begin{array}{l}\text { Secondary sector } \\
\text { (elasticity: }-0,70 \text { ) }\end{array}$} & Income (av. annual gr) & -4.2 & 2.8 & 6.6 & 8.8 & 9.9 \\
\hline & Poverty (\% pop.) & 45.9 & 23.9 & 24.3 & 21.7 & 20.9 \\
\hline \multirow{2}{*}{$\begin{array}{l}\text { Tertiary sector } \\
\text { (elasticity: }-0,90 \text { ) }\end{array}$} & Income (av. annual gr) & 2.3 & 4.8 & 7.2 & 7.9 & 6.9 \\
\hline & Poverty (\% pop) & 36.3 & 23.3 & 21.7 & 19.2 & 16.0 \\
\hline
\end{tabular}

\subsection{SECTORAL STRATEGY FRAMEWORK PROJECTIONS (CADRAGES) AND CONSISTENCY WITH THE PRSP}

452. Methodology: The sectoral strategy frameworks were established in two stages.

- First, the ministries for each sector used a planning tool to quantify their strategies. This framework (known as the "MDGS") shows the cost involved in implementing programs and projects designed to achieve the goals described in Chapter 3. It also points out any financing gap vis-à-vis the domestic and external resources available in the PRSP budgetary framework.

- At a second stage, the sectoral ministries revised their scenarios to bring them in line with the funds released under the core scenario of the PRSP and allocated in the MTBF, as described above. The lowering of budget appropriation ceilings led to a revision of projects and programs and initial targets. This scenario (known as the PRSP) is thus constructed in such a way that no financing gap can persist.

\subsubsection{Education}

\subsubsection{Framework consistent with education strategy/MDGS}

453. Implementation of the education sector strategy is aimed at meeting the MDGS targets of raising enrollment in primary school from 95 percent to 100 percent and the retention rate through to the end of primary school from 43 percent to 80 percent by 2011 . The principal regulatory and pedagogical measures contemplated to improve the quality of the educational system and achieve these results are: (i) granting teachers official status and raising their pay as of 2003; (ii) reducing the grade repletion ratio from 25 percent to 10 percent by 2008 . This scenario, known as the "scenario consistent with education strategy/MDGS," envisages recruiting on average 2,700 teachers and building on average 2,700 classrooms per year in the primary schools sector between 2003 and 2011. 


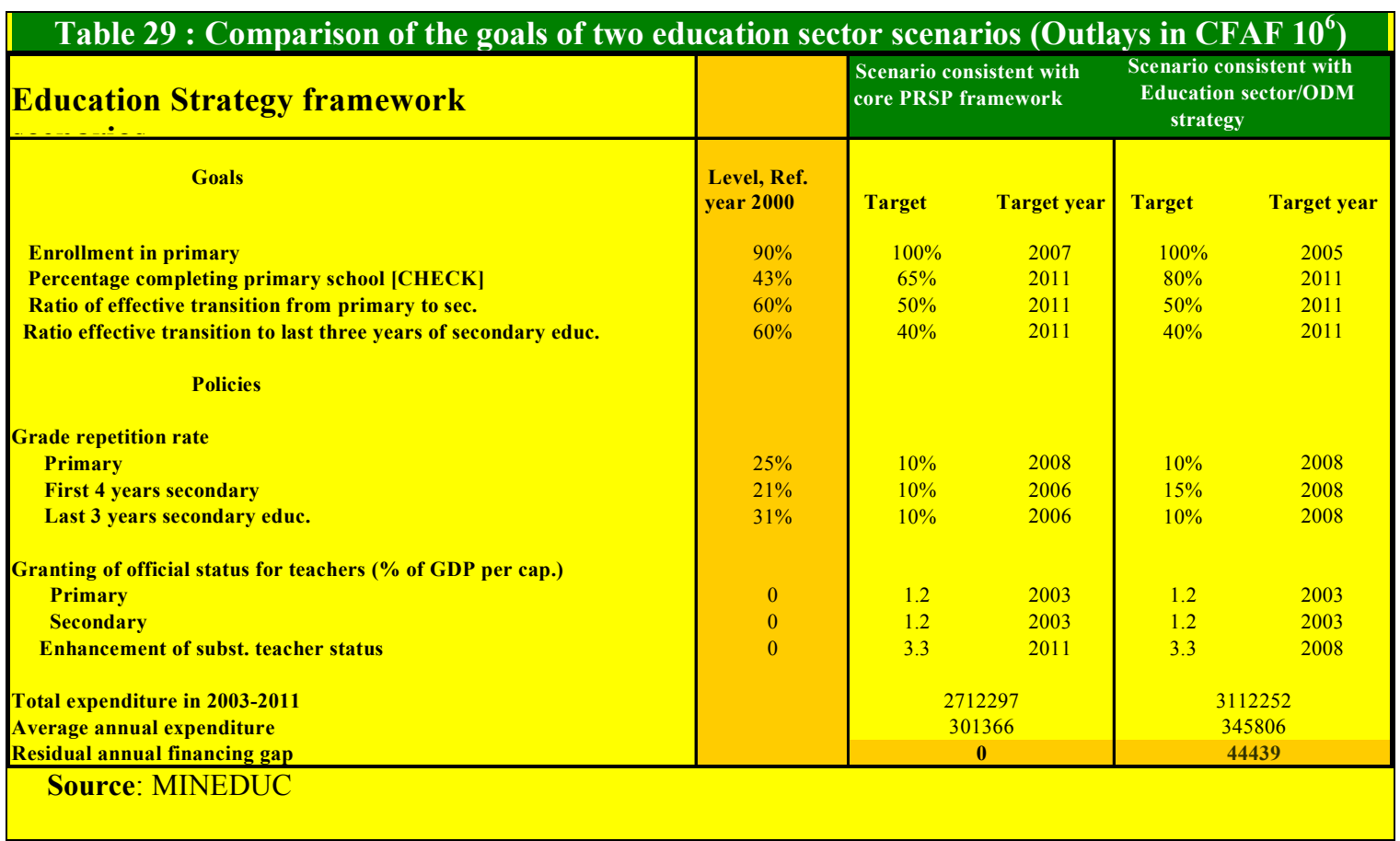

454. The overall cost of the 2003-2011 strategy in this scenario is estimated at CFAF 3,112 billion, i.e., an annual cost of approximately CFAF 346 billion, and an annual financing gap vis-à-vis domestic resources of CFAF 44 billion.

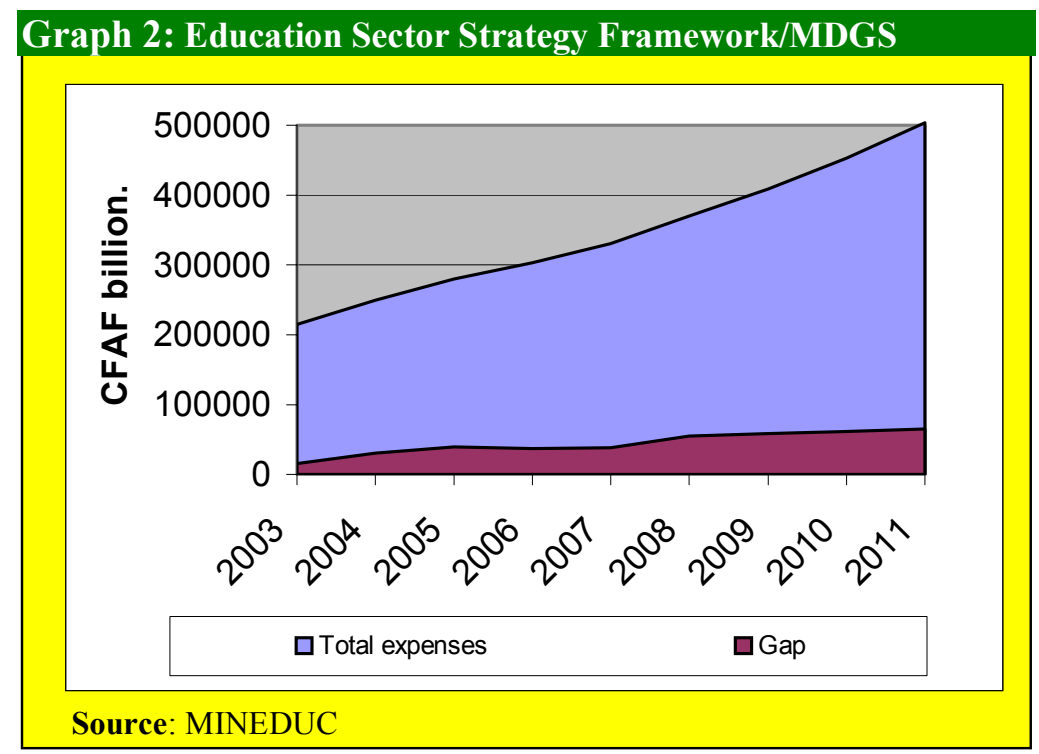




\subsubsection{Framework consistent with the PRSP core scenario}

455. This finding led the authorities to construct a second scenario called the "PRSP scenario," which attempts to keep needs within the budget appropriation ceilings and thereby eliminate the residual requirements gap. This process involved revising goals or procedures. The main changes are that the deadline for achieving universal access is postponed from 2005 to 2007 , and the primary school retention rate will increase to 65 percent, rather than 80 percent, in 2011. The disaggregated MTBF for the education sector, by program and subprogram can be found in Annex 6.

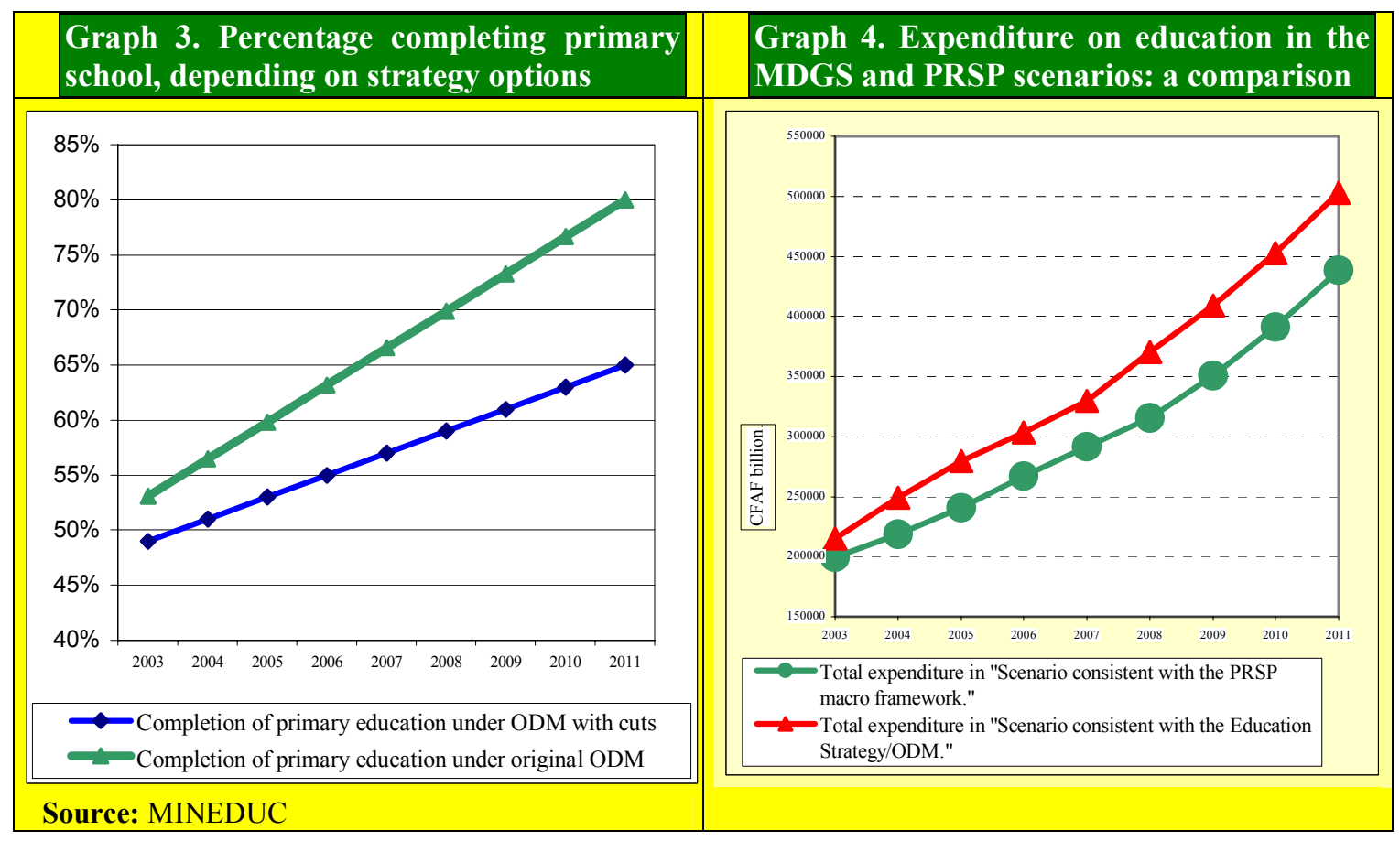




\section{Box 31. The financial simulation model for the education sector}

The model makes it possible to compare resources available with the expenditures attached to education policies, grouped under two headings: current or capital expenditure. The targets and parameters chosen are established for the base year serving as a reference for the simulation (the year 2000), and the decisionmaker adopts the target value at the end of the program (2011) or for a year prior to that in which a target value is supposed to be met (for instance, it is projected that the primary school grade repetition ratio drops from 25 percent to 10 percent by 2008).

Government funds block: the size of GDP and the amount of revenue allocated to education is established according to the macroeconomic model used by the Directorate of Economic Projections.

Current expenditure block: This block covers preschool, primary, secondary (first four years and last three years of general secondary education), technical education, and higher education. By way of illustration, we shall describe the estimation of expenditure at the primary school level related to the targets.

- Estimation of the wage bill: Apart from specifying the "normal" age population and its changes over time, the model is based on the enrollment ratio for the first year of primary school and on the percentage of those enrolling in the first year who continue through to the last year of primary school. These two sets of data, along with the grade repetition ratio (on which appropriate policies can have a decisive impact), make it possible to determine the gross enrollment ratio at the primary school level as well as the number of students enrolled in the education cycle; they are then divided into private and state school pupils. It is then not difficult to estimate, on the basis of the number of pupils, the number of teachers needed each year during the program, by looking at the pupil/teacher ratio and the goals with respect to that ratio. To estimate the wage bill associated with the number of teachers employed, the model has to be fed data on the characteristics of the teachers recruited during the program, with respect to their educational, training and other qualifications, and on the wage policies in place.

- Estimation of other outlays: An adequate overall appropriation ceiling is then established, which will eventually lead to trade-offs between different functions. This determines how overall expenditure should develop, measured against the teachers' wage bill. This method also serves to establish the overall budget for government-run primary schools and the total budget including possible subsidies for private school operating expenses.

Capital expenditure block for classroom construction: First it is necessary to identify changes in the number of class-groups in primary education and in both secondary school cycles. The number of classgroups is then linked to the number of class rooms needed each year to reach the simulated enrollment targets. From the data on the stock of classrooms, one can derive requirements in terms of annual flows. By using an average unit cost of constructing and fitting out a primary and a secondary school classroom, the model then estimates capital expenditure on new classrooms for these education levels.

Comparing expenditure with available funds: On the basis of estimates of likely government revenue and consolidated estimates of current and capital expenditure, it is possible to gauge the country's capacity to finance the different structural educational policy programs simulated by the model.

Source: RESEN Methodology, Alain Mingat and al.

\subsubsection{Health}

456. Initially, the sectoral strategy/MDGs was quantified by using a standard US $\$ 40^{21}$ per person per year estimate of the cost of providing a minimum universal access health care

\footnotetext{
${ }^{21} \mathrm{~J}$. Sachs, Macroeconomics and Health, estimates the cost of a minimum health package at between US\$34 and US\$40 per person.
} 
package of the kind prescribed in the MDGs. This budget was then distributed among programs and projects on the basis of the real needs expressed in the sectoral strategy and a financing gap was established. This appropriation ceiling was then revised to match available resources under the MTBF of the PRSP, in order to eliminate the financing gap.

457. Simulations were carried out using software developed by the authorities, with World Bank assistance, which is based on 13 programs, each of which is subdivided into 54 subprograms. (See the graph below and Annex 6.2.). The model makes it possible to measure changes in operating expenses in relation to capital expenditure, as well as changes in personnel costs and, in particular, changes in doctor/population and nurse/population ratios, and the share of maintenance and capital expenditure on central services compared to such outlays for the decentralized services.

\subsubsection{The sectoral strategy scenario framework/Millennium development goals}

\section{Figure 2. Programming model of the Health Sector}

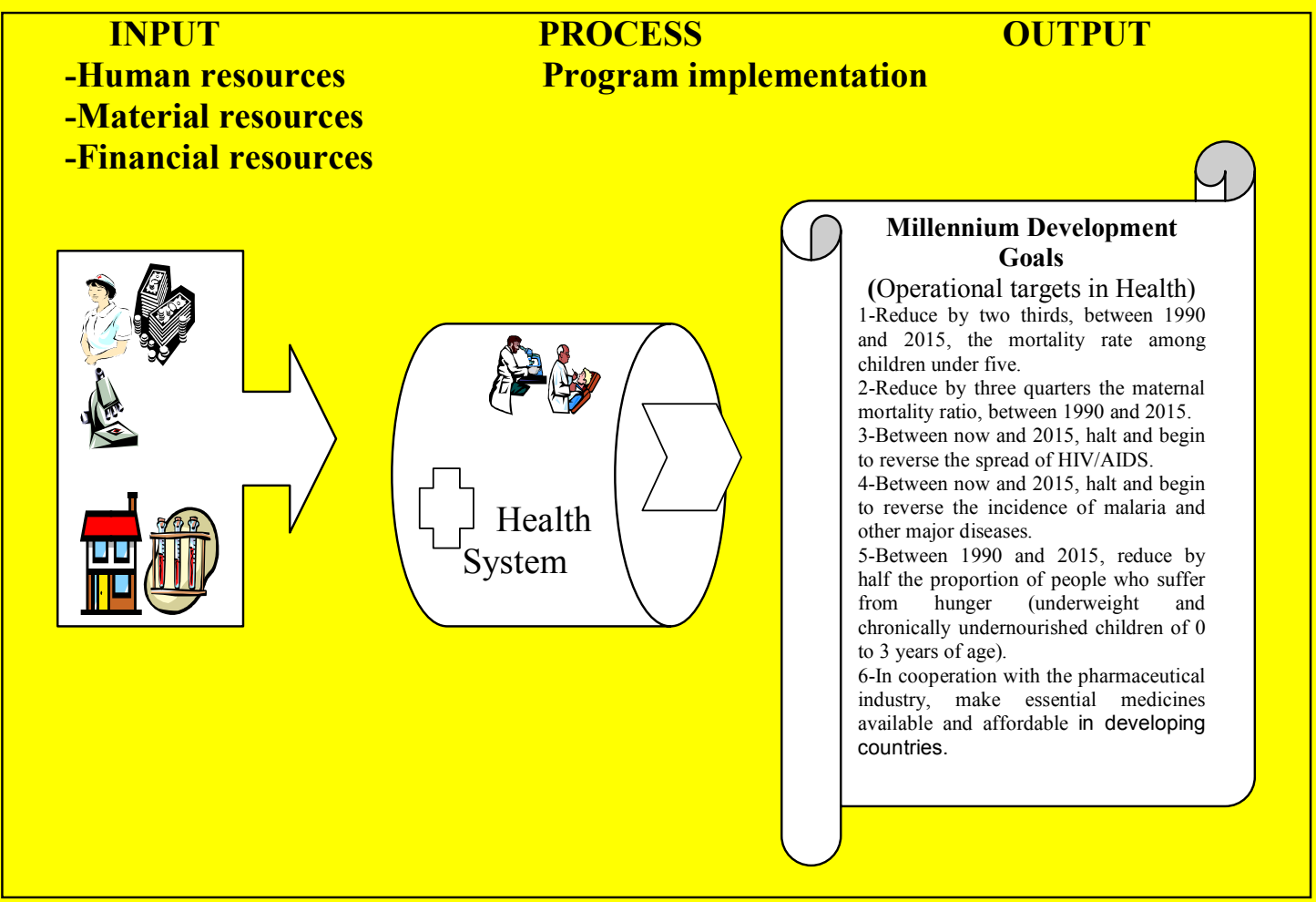

Source: MINSANTE

458. This scenario measures the efforts needed to achieve not only the health sector strategy targets but also the millennium development goals, starting from the situation taken as a reference. This strategy facilitates the deployment of all the human, financial, and material resources needed to improve Cameroon's principal health indicators. Specifically, it envisages reducing by two-thirds the mortality rate among children under five, and cutting the maternal mortality ratio by three-quarters. The spread of AIDS and the incidence of malaria decline in this model by approximately seven points between 2001/02 and 2015. 


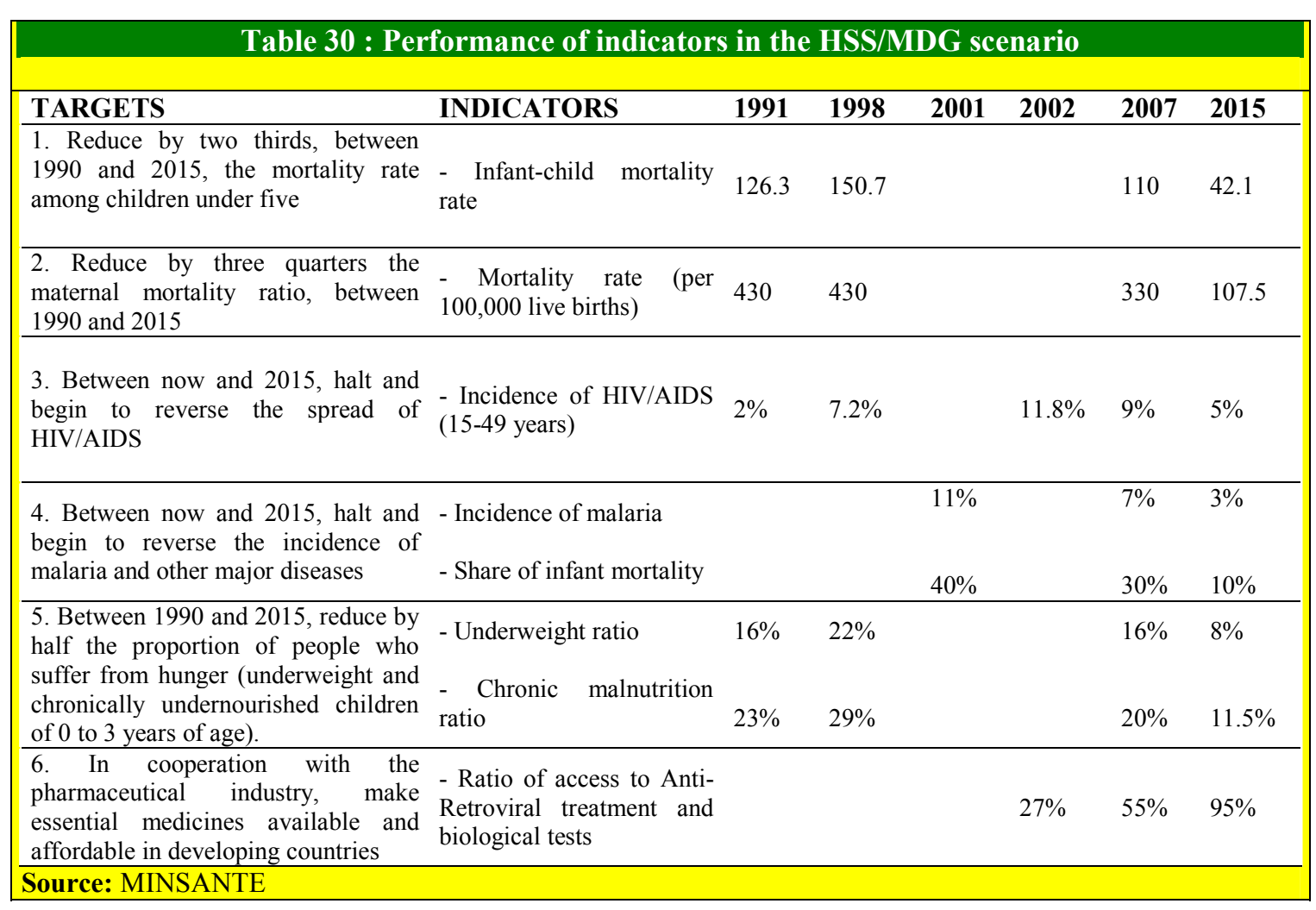

459. The overall cost of implementing this strategy is projected at CFAF 722 billion between 2003 and 2007, which, given the domestic and external funds available, implies an accumulated residual financing requirement of CFAF 115 billion through to end-2007, i.e. an average annual gap of CFAF 29 billion between 2004 and 2007. 


\section{Table 31 : Distribution of funds among the various programs (CFAF 10 ${ }^{6}$ )}

\begin{tabular}{|c|c|c|c|c|c|c|}
\hline Programs & 2003 & 2004 & 2005 & 2006 & 2007 & $\%$ \\
\hline Fighting malaria & 23060 & 53400 & 53702 & 48429 & 51200 & 31.8 \\
\hline Mother, child, and elderly health care & 2409 & 3939 & 4476 & 5119 & 5936 & 3.0 \\
\hline Health promotion & 285 & 883 & 1675 & 1826 & 2284 & 1.0 \\
\hline Strengthening local response/community partnership & 150 & 450 & 547 & 776 & 904 & 0.4 \\
\hline Essential medicines and supplies & 1200 & 1828 & 2339 & 2409 & 3071 & 1.5 \\
\hline Strengthening infrastructure and equipment & 20405 & 25711 & 29428 & 29357 & 34825 & 19.3 \\
\hline Human resource development & 26126 & 27236 & 28633 & 30362 & 32830 & 20.1 \\
\hline Working conditions and facilities & 19453 & 23873 & 25248 & 26918 & 31328 & 17.6 \\
\hline Partnership development & 585 & 892 & 989 & 1089 & 1204 & 0.7 \\
\hline Improvement of administrative procedures & 620 & 969 & 1257 & 1523 & 1854 & 0.9 \\
\hline Structural reform & 400 & 610 & 777 & 941 & 1146 & 0.5 \\
\hline \multirow{2}{*}{$\begin{array}{l}\text { Subsidies for rehabilitation of government agencies (EPA) } \\
\text { Operating and investment credit for second category hospitals }\end{array}$} & 1900 & 2897 & 3689 & 4470 & 5441 & 2.5 \\
\hline & 500 & 763 & 971 & 1177 & 1432 & 0.7 \\
\hline TOTAL EXPENDITURE & 97094 & 143450 & 153731 & 154395 & 173456 & 100 \\
\hline AVAILABLE FUNDS & 97094 & 105477 & 119361 & 133548 & 151018 & \\
\hline \multicolumn{7}{|l|}{ Domestic financing (MINSANTE budget) } \\
\hline & 59442 & 75200 & 90500 & 101500 & 119000 & \\
\hline External financing & 37652 & 30277 & 28861 & 32048 & 32018 & \\
\hline \multicolumn{7}{|l|}{ FINANCING GAP } \\
\hline & 0 & 37973 & 34370 & 20847 & 22438 & 0 \\
\hline
\end{tabular}

\subsubsection{The scenario consistent with the PRSP's macroeconomic hypotheses}

460. As with education, the health sector strategy scenario consistent with the PRSP's macroeconomic hypotheses takes budgetary constraints into account. In this second scenario the cost of the strategy amounts to CFAF 606 billion, or some CFAF 120 billion a year. 


\begin{tabular}{|c|c|c|c|c|c|c|c|}
\hline Programs & 2003 & 2004 & 2005 & 2006 & 2007 & TOTAL & $\%$ \\
\hline Fighting malaria & 23060 & 26951 & 31944 & 36563 & 39072 & 157590 & 26.0 \\
\hline Mother, child, and elderly health care & 2409 & 3353 & 3821 & 4483 & 5224 & 19289 & 3.2 \\
\hline Health promotion & 285 & 883 & 1675 & 2690 & 3278 & 8810 & 1.5 \\
\hline $\begin{array}{l}\text { Strengthening local response/community } \\
\text { partnership }\end{array}$ & 150 & 450 & 547 & 776 & 904 & 2827 & 0.5 \\
\hline Essential medicines and supplies & 1200 & 1828 & 2339 & 2409 & 3071 & 10847 & 1.8 \\
\hline Strengthening infrastructure and equipment & 20405 & 17080 & 20375 & 20147 & 24234 & 102240 & 16.9 \\
\hline Human resource development & 26126 & 27236 & 28633 & 30362 & 32830 & 145188 & 23.9 \\
\hline Working conditions and facilities & 19453 & 21566 & 22345 & 26918 & 31328 & 121609 & 20.1 \\
\hline Partnership development & 585 & 892 & 989 & 1089 & 1204 & 4759 & 0.8 \\
\hline Improvement of administrative procedures & 620 & 969 & 1257 & 1523 & 1854 & 6223 & 1.0 \\
\hline Structural reform & 400 & 610 & 777 & 941 & 1146 & 3874 & 0.6 \\
\hline Subsidies, rehabilitation of government agencies & 1900 & 2897 & 3689 & 4470 & 5441 & 18397 & 3.0 \\
\hline $\begin{array}{l}\text { Operating and investment credit for second } \\
\text { category hospitals }\end{array}$ & 500 & 763 & 971 & 1177 & 1432 & 4842 & 0.8 \\
\hline TOTAL EXPENDITURE & 97094 & 105477 & 119361 & 133548 & 151018 & 606498 & 100.0 \\
\hline AVAILABLE FUNDS & 97094 & 105477 & 119361 & 133548 & 151018 & 606498 & \\
\hline Domestic financing (MINSANTE budget) & 59442 & 75200 & 90500 & 101500 & 119000 & 445642 & \\
\hline External financing & 37652 & 30277 & 28861 & 32048 & 32018 & 160856 & \\
\hline FINANCING GAP & 0 & 0 & 0 & 0 & 0 & & \\
\hline
\end{tabular}

461. Expenditure cuts would undoubtedly entail sacrificing part of the goals, as the Table below shows. Progress in indicators such as the infant mortality rate or the maternal mortality rate, which reflect the overall performance of the health system, is slower than in the previous scenario. For instance, the infant-child mortality rate is $75 / 1000$ as opposed to $42,1 / 1000$ in the foregoing exercise: a 32.9 point difference. The reversal of widespread endemic diseases, such as AIDS and malaria, is also weaker. The incidence of AIDS is projected at 9 percent in 2015 , compared with 5 percent in the previous scenario, and that of malaria is shown as 8 percent, rather than 3 percent. The health indicators can only be improved by substantial investment in the health sector. The major gaps between millennium development goals and the outcomes projected in the scenario consistent with the macroeconomic hypotheses of the PRSP underscore the need for an active and sustained effort to raise additional funds. 
Table 33 : Summary of how far each scenario falls short of the millennium development goals

\begin{tabular}{|c|c|c|c|c|c|c|c|c|}
\hline \multirow{2}{*}{ TARGETS } & \multirow{2}{*}{ INDICATORS } & \multirow[t]{2}{*}{$\begin{array}{l}\text { Base level } \\
1998\end{array}$} & \multicolumn{3}{|c|}{2007} & \multicolumn{3}{|c|}{2015} \\
\hline & & & MDGS & PRSP & Shortfall & MDGS & PRSP & Shortfall \\
\hline $\begin{array}{l}\text { 1. Reduce by two thirds, between } \\
1990 \text { and } 2015 \text {, the mortality rate } \\
\text { among children under five. }\end{array}$ & $\begin{array}{l}\text {-Infant-child } \\
\text { mortality rate (per } \\
1000)\end{array}$ & 150.7 & 110 & 130 & -20 & 42.1 & 75 & -32.9 \\
\hline $\begin{array}{l}\text { 2. Reduce by three quarters the } \\
\text { maternal mortality ratio, between } \\
1990 \text { and } 2015 \text {. }\end{array}$ & $\begin{array}{lr}- & \text { Maternal } \\
\text { mortality } & \text { rate (per } \\
100,000 & \text { live } \\
\text { births) } & \\
\end{array}$ & 430 & 330 & 400 & -70 & 107.5 & 350 & -242.5 \\
\hline $\begin{array}{l}\text { 3. Between now and } 2015 \text { halt and } \\
\text { begin to reverse the spread of } \\
\text { HIV/AIDS. }\end{array}$ & $\begin{array}{l}\text { - Incidence of } \\
\text { HIV/AIDS (15-49 } \\
\text { years) }\end{array}$ & 2002 & 9 & 11 & -3 & 5 & 9 & -4 \\
\hline \multirow{3}{*}{$\begin{array}{l}\text { 4. Between now and } 2015 \text { halt and } \\
\text { begin to reverse the incidence of } \\
\text { malaria and other major diseases. }\end{array}$} & \multirow{3}{*}{$\begin{array}{l}\text { - Incidence of } \\
\text { malaria (\%) } \\
\text { - Share of infant } \\
\text { mortality }(\%)\end{array}$} & 2002 & & & & & & \\
\hline & & 11 & 7 & 10 & -3 & 3 & 8 & -5 \\
\hline & & 40 & 30 & 35 & -5 & 10 & 25 & -15 \\
\hline \multirow{3}{*}{$\begin{array}{l}\text { 5. Between } 1990 \text { and } 2015 \text {, reduce } \\
\text { by half the proportion of people } \\
\text { who suffer from hunger } \\
\text { (underweight and chronically } \\
\text { undernourished children of } 0 \text { to } 3 \\
\text { years of age). }\end{array}$} & \multirow{3}{*}{$\begin{array}{lr}- & \\
\text { ratio } & \\
& \\
- & \text { Chronic } \\
\text { malnutrition ratio }\end{array}$} & 1998 & & & & & & \\
\hline & & 22 & 16 & 19 & -3 & 8 & 12 & -4 \\
\hline & & 29 & 20 & 23 & -3 & 11.5 & 17 & -5.5 \\
\hline $\begin{array}{l}\text { 6. In cooperation with the } \\
\text { pharmaceutical industry, make } \\
\text { essential medicines available and } \\
\text { affordable in developing countries. }\end{array}$ & $\begin{array}{l}\text { - Ratio of access to } \\
\text { Anti-Retroviral } \\
\text { treatment and } \\
\text { biological tests } \\
(\%)\end{array}$ & 2002 & 55 & 30 & -25 & 95 & 35 & -55 \\
\hline
\end{tabular}

\subsubsection{Road infrastructure}

462. The procedure adopted consisted of:

- Establishing goals in terms of the length and maintenance of the network for asphalted roads, roads on priority land, high priority rural roads, and non-priority roads;

- Quantifying the costs of implementing the planned activities based on unit costs of roadworks; and

- Comparing the resulting expenditure with total available resources. 


\section{Figure.3. Methodological framework for planning}

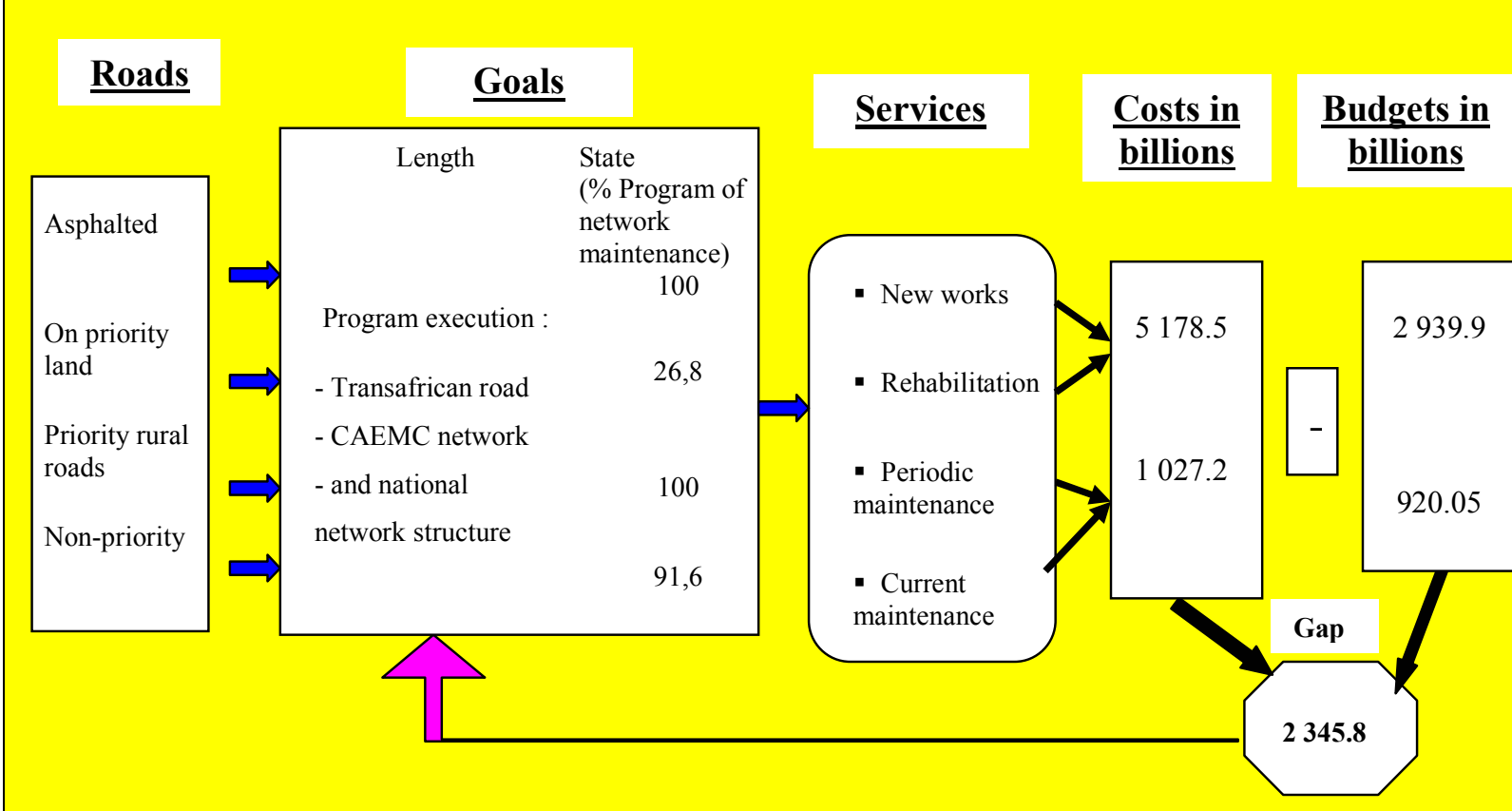

The works derive from the established goals (which can still be adjusted). The diffference between the cost of these works and the budget indicates either the amount of extra financing needed or the budgetary surplus, as the case may be.

\subsubsection{Framework consistent with the MDGs}

463. The MDGS strategy aims to accomplish 100 percent of the goals described in the strategy, which entail adding approximately $7,070 \mathrm{~km}$ of roads and rehabilitating $29,500 \mathrm{~km}$, while maintenance is to be performed on the whole of the road network by 2015. This scenario has a financing gap over the next 13 years (2003-2015) of approximately CFAF 2,345 billion.

\subsubsection{Road infrastructure framework consistent with the PRSP macroeconomic framework}

464. To take available resources into account, the authorities drew up a second framework, which revises the schedule and the volume of works. To that end, the additional road building refers to the Transafrican highway and the CAEMC network only. The rehabilitation investment will be for $23,300 \mathrm{~km}$, of which $1,651 \mathrm{~km}$ will entail new construction. 


\begin{tabular}{|c|c|c|c|c|c|c|c|c|c|c|}
\hline \multirow{3}{*}{ 营 } & \multirow{3}{*}{ Services } & \multirow{2}{*}{\multicolumn{2}{|c|}{ Goals }} & \multirow{2}{*}{\multicolumn{3}{|c|}{$\begin{array}{l}\text { Volume of services (in } \\
\qquad \mathrm{km} \text { ) }\end{array}$}} & \multicolumn{3}{|c|}{ Costa } & $\begin{array}{c}\text { Overall } \\
\text { gaps }\end{array}$ \\
\hline & & & & & & & \multicolumn{4}{|c|}{ (in CFAF billions) } \\
\hline & & Expansion & $\begin{array}{l}\text { State } \\
(\% \text { maint. } \\
\text { progr. })\end{array}$ & 2003 & 2007 & 2015 & 2003 & 2007 & 2015 & \\
\hline \multirow{4}{*}{$\begin{array}{l}0 \\
0 \\
\sum_{0} \\
.0 \\
0 \\
0\end{array}$} & New construction & Program & \multirow{4}{*}{100} & 544.0 & 544.0 & 544.0 & 136.0 & 136.0 & 136.0 & \multirow{4}{*}{2345.8} \\
\hline & Rehabilitation & $\begin{array}{l}\text { execution: } \\
\text { Trancafrican }\end{array}$ & & 2739.0 & 2789.0 & 2789.0 & 322.97 & 322.97 & 190.84 & \\
\hline & $\begin{array}{l}\text { Periodic } \\
\text { maintenance }\end{array}$ & \multirow{2}{*}{$\begin{array}{l}\text { highway, the } \\
\text { CAEMC } \\
\text { network, and the } \\
\text { structure of the } \\
\text { national network }\end{array}$} & & 2839.0 & 2839.0 & 3691.0 & 30.47 & 30.75 & 32.75 & \\
\hline & $\begin{array}{l}\text { Current } \\
\text { maintenance }\end{array}$ & & & 10994 & 21338 & 43984 & 18.36 & 35.34 & 72.50 & \\
\hline \multirow{4}{*}{ 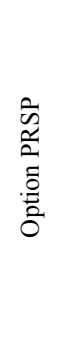 } & New construction & \multirow{4}{*}{$\begin{array}{l}\text { Program } \\
\text { execution: } \\
\text { Transafrican } \\
\text { highway, and the } \\
\text { CAEMC network }\end{array}$} & \multirow{4}{*}{75} & 127.0 & 127.0 & 127.0 & 31.75 & 31.75 & 31.75 & \multirow{4}{*}{0} \\
\hline & Rehabilitation & & & 1623.0 & 2070.0 & 1627.0 & 187.94 & 239.71 & 188.41 & \\
\hline & $\begin{array}{l}\text { Periodic } \\
\text { maintenance }\end{array}$ & & & 2700.0 & 2700.0 & 3610.0 & 34.87 & 34.87 & 42.29 & \\
\hline & $\begin{array}{l}\text { Current } \\
\text { maintenance }\end{array}$ & & & 11059 & 17551 & 31802 & 18.29 & 28.78 & 51.79 & \\
\hline
\end{tabular}

\subsection{FINANCING THE POVERTY REDUCTION STRATEGY}

\subsubsection{The overall cost of the strategy}

465. The strategy described in the PRSP is integrated and its seven core components (axes) cover almost all the principal sectors. In particular, the strategies in priority sectors describe all the activities in those sectors during the PRSP period, and not just "poverty expenditure." Even in the non-priority sectors, all the sector programs are henceforth encompassed by the PRSP framework. That being so, the authorities opted to quantify all budget estimates rather than have a separate quantification of "poverty expenditure" and to provide a breakdown of the costs of the priority sector programs (Cf. the education sector and health sector frameworks, etc.).

466. To summarize: the estimations show that the total cost of the strategy rises from CFAF 950.5 billion in 2003 to CFAF 1,360 billion in 2007, an average annual increase of approximately 9 percent. The priority sector subtotal is projected to increase from CFAF 568 billion in 2003 to CFAF 866 billion in 2007, an average annual increase of 11 percent. The priority sectors share in this total is projected to rise from 56 percent in 2003 to 64 percent in 2007, a net gain of 8 points over the period. These increases in priority expenditure, without crowding out other sectors, are possible thanks to debt forgiveness. That explains why total budget estimates (ministerial remits, other items, and debt servicing) are only projected to increase by approximately 5 percent over the whole period, that is to say, two points less than nominal GDP. Domestic resources are projected to cover approximately 92 percent of the ministry chapters. 
467. The authorities intend to work with development partners to raise external funds and to improve coordination of the assistance provided to ensure that it matches the strategy's priorities. That will continue as part of the consultation process used to draw up the PRSP and of the strategy implementation follow-up process.

\subsubsection{Financing the strategy}

468. Mobilizing domestic resources. Tax revenue will continue to provide the bulk of the domestic funding of the strategy. As mentioned earlier, the strategy for raising tax revenue will focus on three key areas:

- Expansion of the tax base. This will entail the following measures: (i) At the legislative level: pruning exemptions, especially exonerations from VAT and income tax (eliminating the income tax exemption in return for reinvestment); (ii) At the administrative level: systematic identification and registration of new taxpayers, activation of link-ups with Customs (control of imports files) and with the Budget office (control of files on government suppliers), the gathering of data from large public and private enterprises, and securing the loyalty of registered taxpayers, thereby increasing the tax return ratio; (iii) At the organizational level: raising the effectiveness of existing security programs (drills, forets) and expediting implementation of the recently created mining revenue security program; and (iv) introduction of the income tax reform in 2004, along with the property tax.

- Rationalization of tax audits. This should ensure that all enterprises are audited within a four-year period. Here the emphasis will be on training personnel, using less cumbersome and more profitable audit procedures, and allowing inspectors to specialize in certain broad categories of business activities.

- Improved collection and, especially, application of the new procedures issued in the tax procedures manual.

469. As regards customs, the authorities intend to improve on-site inspection and documentary audits with respect to merchandise, to tighten supervision of exonerations and special status traffic, to step up one-stop shop activities, especially through computerization of services, and to strengthen the role of the joint Tax and Customs unit in gathering information and in joint audits.

470. External funding. The government intends to work with development partners in order to raise external funds and improve coordination of the various kinds of assistance, taking into account the priorities of the strategy. This partnership will continue in the context of the consultation process put in place for the drafting of the PRSP and as part of the follow up to strategy implementation. In addition, the government will proceed to create all the conditions needed to encourage private sector participation in the financing of the strategy. 
Chapter 4: Macroeconomic and sectoral framework

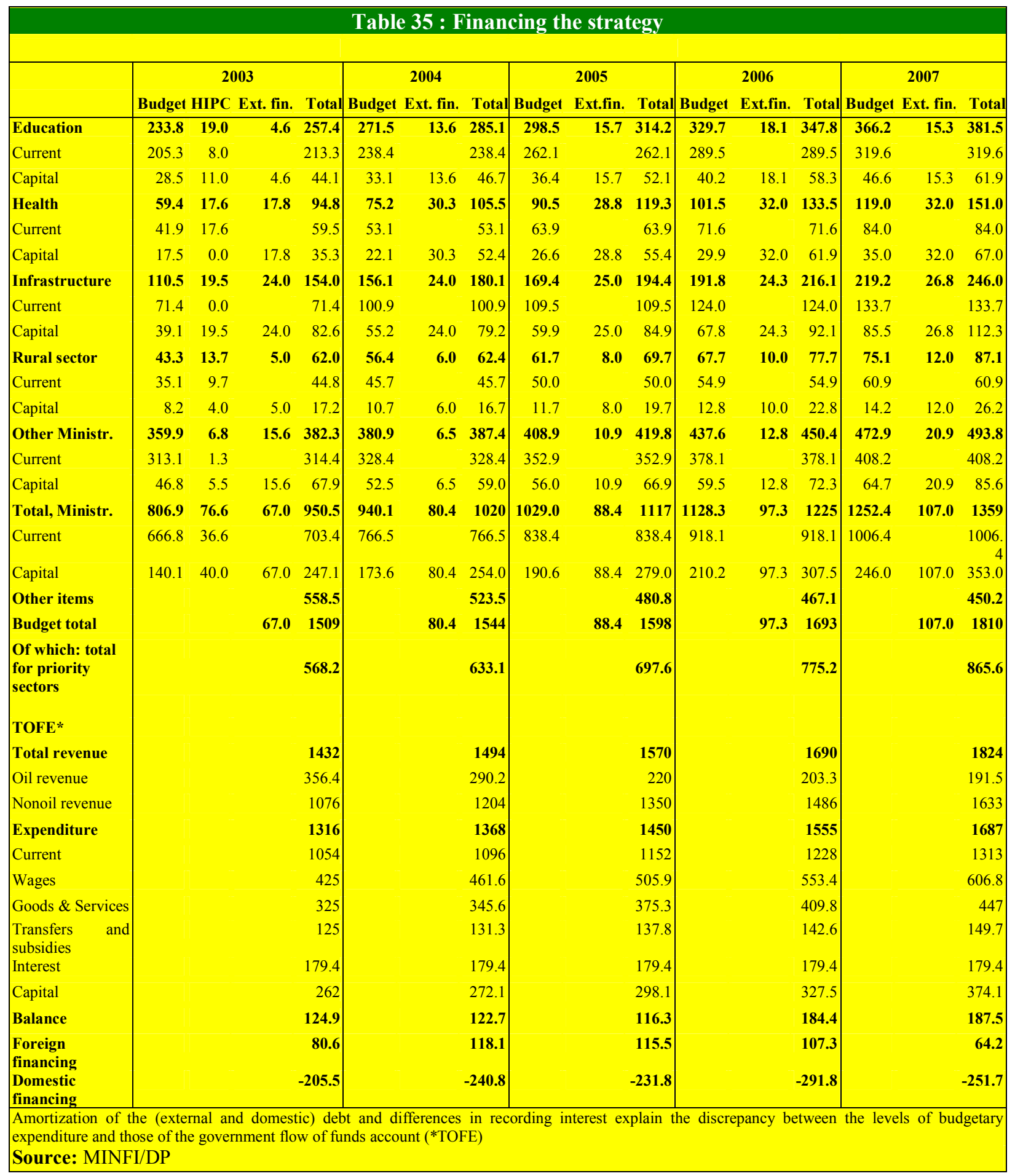




\subsection{RISK ANALYSIS AND ALTERNATIVE SCENARIOS}

471. The PRSP scenario is based on a certain number of key hypotheses with respect to the international environment, economic and social policies, and effective execution of programs. Adverse external shocks such as the fall in oil prices or a contraction in the expected flow of funds from abroad, ongoing domestic structural rigidities (power shortages, weak foreign investment flows, unsatisfactory execution of the privatization program, or a low ratio of absorption of the funds allocated in the budget) could reduce growth and hence the impact on poverty. Such a contraction in the budget could lead the government to make difficult adjustments in sectoral outlays, which could hamper the achievement of PRSP goals. The following sensitivity analyses make it possible to gauge the impact of these risk factors on growth and poverty profiles as well as the implications for availability of funding in the priority sectors.

\subsubsection{Methodology}

472. The impact analyses are based on the core scenario. They entail modifying the hypotheses regarding PRSP risk factors and measuring the effects of those modifications in terms of deviation from the growth rate and from the budget allocations in the MTBF. The impacts on the poverty ratio are inferred, as above, from the rate of growth, by using the elasticities method. The analysis is expanded to take into account household income distribution effects. To do that, an interface was constructed between the macroeconometric endogenous growth model and the 123PRSP model (a simple computable general equilibrium model) which comprises a detailed "households" module with 10 groups of households (deciles). The inference regarding the behavior of monetary inequalities is made by comparing the increase in the income of the poorest 20 percent of households with that of the richest 20 percent. The main findings of the analysis are shown in the Table below.

\section{Box 32. Methodology employed in the impact analysis}

The 123PRSP model was obtained by linking the 123 module (a simple computable general equilibrium model) and the ECAM II household module to the macroeconomic projections model. This combination of tools makes it possible to gauge the impact of external shocks (such as the terms of trade), changes in fiscal policy (such as a change in public expenditure), or structural reforms (of physical, financial, and social infrastructure, for instance) on medium-term growth, relative prices, and the distribution of household income. The different modules of the 123PRSP model are described at greater length in an annex.

Source: MINFI/ DP and DSCN

\subsubsection{Impact of adverse shocks on the terms of trade}

473. The Cameroonian economy remains highly dependent upon a small number of primary sector exports. In 2000, not counting crude oil, represented approximately 50 percent of total exports. International prices for these products are particularly volatile, which constitutes a risk for the producer and affects production paths and hence the variance of national income. The Table below shows contrasting trends for the principal products over the past 20 years (+20 percent and 38 percent, respectively, for cocoa and bananas and -14 percent, -35 percent, and -20 percent, respectively, for oil, coffee, and cotton). The variation coefficients (ratios of standard variation to average prices) are particularly high, reflecting the extremely volatile nature of world prices for these products. 


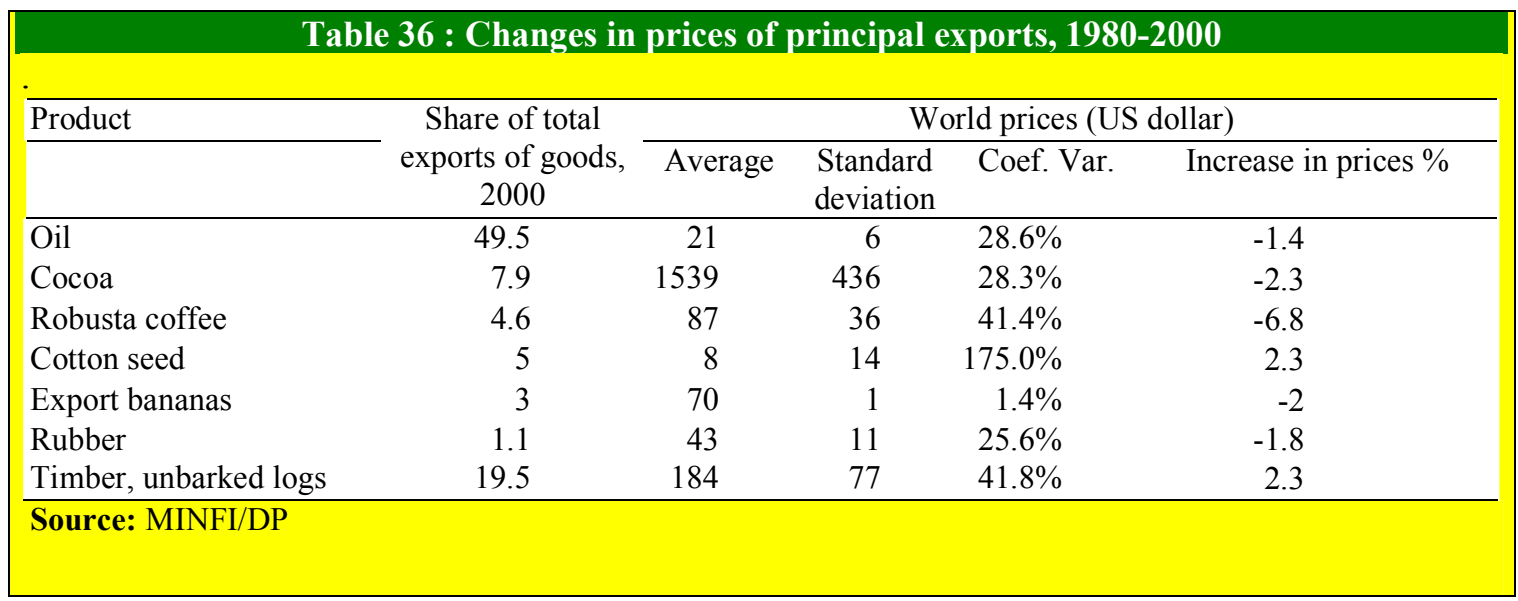

474. The above Table shows that all products, including oil and cocoa, have undergone major fluctuations over the past five years. The crises in Iraq and Côte d'Ivoire are likely to bring about significant shifts in the prices of these products in 2003 and, possibly, in 2004-2005. If trends so far observed in 2003 continue, prices are likely to remain high. If not, if the Iraqi crisis is solved quickly, the oil market could be affected by excess capacity and stable or declining prices in 2003-2005. Rapid settlement of the crisis in Côte d'Ivoire would create similar conditions in the cocoa market.

475. To gauge the effect of these shocks on the PRSP scenario, two simulations were carried out. In the first, ongoing tensions in 2003, settled in early 2004, would produce increases in the prices of oil and cocoa in 2003. That could translate into an increase in GDP of approximately 1.4 percent in 2003 and 2004, followed by a reversal of the trend in 2005-2007 as prices fall. All in all, the average effect over the period remains moderate, with an overall gain of 0.11 percent in annual growth. Thus budget adjustments would be negligible.

476. In the second simulation, the crises are over quickly, and prices for these products decline in 2003. The results indicate a substantial drop in growth of approximately one fifth of a point per annum, tapering off by 2007. Own revenue losses are significant (averaging 38 billion per annum compared to the core scenario), obliging the authorities to make proportional cuts in the budget allocations for the priority sectors. The "social effects" - on distribution and poverty-are also substantial. Thus, household incomes fall by an accumulated 5 points in 2004-2007. The decline is more pronounced ( -1.5 points of deviation) for the poorest 20 percent of households than it is for the richest 20 percent. With the reduction in the average rate of growth, poverty reduction gains are smaller (by about 1.2 points) in 2003-2007, putting the poverty rate at 35.8 percent in 2007 , as opposed to the 34.6 percent contemplated in the core scenario. Even stronger growth after 2007 would therefore be needed to offset this slippage and attain millennium goals by 2015 .

\subsubsection{Effects of a contraction in the expected flow of funds from abroad or of persisting fund absorption problems.}

477. Implementation of the PRSP is based on the hypothesis that concomitant financing requirements are covered by external assistance. In its core framework projections (cadrage central), the government has already pointed to a major domestic effort to reduce residual financing requirements to a very realistic level. However, it remains possible that those residual funds do not materialize, or that structural problems continue to hamper effective 
absorption of them in programs and projects. To assess the importance of these concerns, two simulations have been run to show (i) the effects of a halving of "expected" external financing and (ii) the effects of weak absorption of funds. The main difference between the two scenarios is that one entails a contraction of the whole budget contemplated in the MTBF, while the other entails different levels of cuts in the sectoral budget allocation ceilings because of differences in the absorption rates of the various sectors (education, health, etc.) and in the nature of the expenditure (current or capital).

478. As summarized in the Table below, a halving of expected resources would reduce growth by approximately 0.6 points per annum in 2004-2007, which would mean that average annual growth for the period would be 5 percent, rather than 6 . Taking into account the adjustments that would have to be made to balance the government's fund flow account (TOFE) following the new financing constraint, the budget ceiling (domestic and external funds combined) contemplated in the core budget projections would have to be revised downwards by approximately 110 billion per annum for the whole period. This would lower the likelihood of carrying out sectoral programs and hence the chances of achieving the strategy's goals.

479. The effects of persisting fund absorption problems are equally dire. The scenario assumes sectoral absorption capacities that would result in an average rate of absorption of 94 percent for current expenditure and 66 percent for capital expenditure over the whole period. The average rate of growth would be 4.4 percent (compared with 5 percent in the foregoing case) and budgetary adjustments would be even more marked.

\subsubsection{Ongoing problems in the electric power sector.}

480. Persisting structural problems in the energy sector would impair the chances of lowering production costs and raising the competitiveness of industry, and hence the possibility of achieving deeper and more diversified growth. To assess the importance of these problems, the effects of only a "timid" revival of the sector were simulated by positing an average increase in energy supply of only half the planned level ( 3 percent instead of 6 percent) and a 5 billion a year cut in the budget allocation for the sector. The consequences in terms of GDP are almost as significant as in the case of an adverse shock in the terms of trade. The average rate of growth falls by approximately 0.4 of a point per year, and average annual own revenue also falls short by some 23 billion compared to the scenario. The domestic budget would therefore have to be trimmed by the same amount or the losses would have to be offset with alternative resources in order to balance the fiscal accounts. A downward adjustment in expenditure of that magnitude would contribute to a further decline in the medium-term growth rate, impairing the chances of achieving the strategy's goals.

481. Finally, the analysis contemplates each of the risk factors separately. However some of these factors interact and simultaneous developments are quite likely. For instance, the availability of foreign funds is linked to the capacity to absorb those funds. Development partners would certainly be ill inclined to schedule additional resources if they perceive that structural problems are hampering absorption and therefore effective use of them. Likewise, power supply problems and vulnerability to the terms of trade are both linked to the structural shortcomings that limit competitiveness and the possibility of placing the economy on a more sustainable footing. The foregoing analysis shows that any combination of these "risk factors" would take growth back to the average rate of the past few years, jeopardizing the 
achievement of the PRSP goals. That means that the government must strive even harder to implement its program of structural reforms if the strategy is to succeed.

\begin{tabular}{|c|c|c|c|c|c|c|c|c|c|c|c|c|c|c|c|c|}
\hline & & & Tabl & le 37 & : Pri & incip & pal i & dis & itors & & & & & & & \\
\hline & & Estima & & & & & & & & Project & ctions & & & & & \\
\hline & 2000 & 2001 & 2002 & 2003 & 2004 & 2005 & 2006 & 2007 & 2008 & 2009 & 2010 & 2011 & 2012 & 2013 & 2014 & 2015 \\
\hline & & & & & & & & & FAF bill & lions & & & & & & \\
\hline GDP at current prices (current & 6074 & 6474 & 6869 & 7386 & 7887 & 8406 & 9008 & 9729 & 10544 & 11468 & 12497 & 13642 & 14893 & 16259 & 17752 & 19403 \\
\hline GDP at constant prices(CFAF & 3950 & 4139 & 4313 & 4507 & 4727 & 4981 & 5268 & 5584 & 5935 & 6326 & 6755 & 7226 & 7728 & 8267 & 8845 & 9472 \\
\hline & & & & & & & & Annual & growth & $($ in $\%)$ & & & & & & \\
\hline GDP/per capita & 2.0 & 2.0 & 1.4 & 1.7 & 2.1 & 2.6 & 3.0 & 3.2 & 3.5 & 3.8 & 4.0 & 4.2 & 4.2 & 4.2 & 4.2 & 4.3 \\
\hline Consumption per capita & 0.2 & -2.1 & 2.8 & 1.3 & 2.0 & 2.1 & 2.2 & 2.2 & 2.5 & 2.9 & 3.0 & 3.4 & 3.7 & 3.7 & 3.8 & 3.7 \\
\hline GDP at constant prices & 4.8 & 4.8 & 4.2 & 4.5 & 4.9 & 5.4 & 5.8 & 6.0 & 6.3 & 6.6 & 6.8 & 7.0 & 7.0 & 7.0 & 7.0 & 7.1 \\
\hline Prices & & & & & & & & & & & & & & & & \\
\hline GDP deflator & 3.2 & 1.7 & 1.8 & 2.9 & 1.8 & 1.2 & 1.3 & 1.9 & 2.0 & 2.0 & 2.0 & 2.1 & 2.1 & 2.1 & 2.1 & 2.1 \\
\hline Consumer prices & -1.0 & 1.3 & 2.8 & 2.8 & 2.6 & 2.1 & 2.0 & 2.0 & 2.0 & 2.0 & 2.0 & 2.0 & 2.0 & 2.0 & 2.0 & 2.0 \\
\hline Prices of exports & 24.3 & 2.7 & 1.4 & 2.2 & -2.4 & -2.1 & -1.1 & 1.3 & 1.7 & 1.9 & 1.9 & 1.9 & 1.9 & 1.8 & 1.8 & 1.8 \\
\hline Prices of imports & 3.4 & 2.7 & 3.2 & 0.2 & 0.1 & 1.5 & 1.7 & 2.0 & 2.0 & 2.0 & 2.0 & 2.0 & 2.0 & 2.0 & 2.0 & 2.0 \\
\hline Terms of trade & 20.9 & 0.0 & -1.8 & 2.0 & -2.5 & -3.6 & -2.7 & -0.6 & -0.4 & -0.2 & -0.2 & -0.2 & -0.2 & -0.3 & -0.3 & -0.3 \\
\hline & & & & & & & As a & percen & ntage of & f GDP $(\%$ & & & & & & \\
\hline GDP at market prices & & & & & & & & & & & & & & & & \\
\hline Primary sector & 42.7 & 42.4 & 42.9 & 42.4 & 42.3 & 42.4 & 42.3 & 41.9 & 41.3 & 40.6 & 39.9 & 39.1 & 38.4 & 37.8 & 37.3 & 36.8 \\
\hline Secondary sector & 19.6 & 17.7 & 16.9 & 16.4 & 15.1 & 14.6 & 14.3 & 14.1 & 14.1 & 14.1 & 14.1 & 14.2 & 14.2 & 14.4 & 14.6 & 14.9 \\
\hline Tertiary sector & 34.9 & 37.1 & 37.4 & 38.4 & 39.8 & 40.2 & 40.6 & 41.2 & 41.8 & 42.5 & 43.2 & 43.9 & 44.5 & 45.0 & 45.3 & 45.5 \\
\hline Components of demand & & & & & & & & & & & & & & & & \\
\hline Consumption & 79.6 & 81.0 & 83.4 & 83.1 & 83.7 & 83.8 & 83.4 & 82.3 & 81.2 & 80.1 & 79.0 & 78.1 & 77.6 & 77.1 & 76.7 & 76.0 \\
\hline Private & 68.9 & 69.7 & 71.7 & 71.2 & 71.7 & 71.6 & 71.1 & 70.0 & 69.0 & 68.0 & 67.0 & 66.3 & 65.9 & 65.6 & 65.4 & 64.9 \\
\hline Public & 10.7 & 11.3 & 11.8 & 11.9 & 12.0 & 12.1 & 12.2 & 12.2 & 12.2 & 12.1 & 12.0 & 11.8 & 11.6 & 11.5 & 11.3 & 11.1 \\
\hline Gross Fixed Capital Formation & 17.1 & 18.1 & 18.1 & 18.3 & 18.5 & 18.9 & 19.3 & 19.7 & 20.3 & 20.7 & 21.2 & 21.7 & 22.2 & 22.7 & 23.0 & 23.4 \\
\hline Exports, Goods \& services & 31.3 & 29.5 & 28.0 & 27.5 & 26.1 & 25.1 & 24.6 & 24.7 & 24.9 & 25.1 & 25.4 & 25.8 & 25.8 & 25.9 & 26.1 & 26.5 \\
\hline Imports, Goods \& services & 28.0 & 28.6 & 29.5 & 28.9 & 28.3 & 27.8 & 27.3 & 26.7 & 26.3 & 25.9 & 25.6 & 25.5 & 25.5 & 25.6 & 25.8 & 25.9 \\
\hline Government & & & & & & & & & & & & & & & & \\
\hline Revenue, excl. grants & 21.6 & 19.3 & 19.7 & 19.4 & 18.9 & 18.7 & 18.8 & 18.8 & 18.6 & 18.6 & 18.4 & 18.4 & 18.5 & 18.6 & 18.6 & 18.4 \\
\hline Oil & 7.2 & 5.2 & 5.4 & 4.8 & 3.7 & 2.6 & 2.3 & 2.0 & 1.9 & 1.7 & 1.5 & 1.3 & 1.1 & 1.0 & 0.8 & 0.7 \\
\hline Nonoil & 14.4 & 14.1 & 14.4 & 14.6 & 15.3 & 16.1 & 16.5 & 16.8 & 16.7 & 16.9 & 16.9 & 17.1 & 17.4 & 17.7 & 17.8 & 17.7 \\
\hline Expenditure & 17.0 & 18.4 & 17.7 & 17.8 & 17.3 & 17.2 & 17.3 & 17.3 & 17.6 & 17.5 & 17.6 & 17.6 & 17.6 & 17.6 & 17.4 & 17.2 \\
\hline Current & 13.2 & 14.8 & 14.7 & 14.3 & 13.9 & 13.7 & 13.6 & 13.5 & 13.4 & 13.1 & 12.9 & 12.7 & 12.5 & 12.2 & 12.1 & 11.9 \\
\hline Capital & 3.6 & 3.6 & 2.8 & 3.5 & 3.5 & 3.5 & 3.6 & 3.8 & 4.2 & 4.4 & 4.7 & 4.9 & 5.1 & 5.4 & 5.4 & 5.3 \\
\hline Expenditure, excl. interest & 15.0 & 14.9 & 14.8 & 15.4 & 15.3 & 15.7 & 15.9 & 16.2 & 16.6 & 16.8 & 16.9 & 17.0 & 17.1 & 17.2 & 17.1 & 16.9 \\
\hline Overall balance, cash b. (excl. grants) & 2.4 & -1.2 & -3.6 & 1.1 & 0.9 & 0.8 & 1.5 & 1.4 & 1.0 & 1.1 & 0.9 & 0.9 & 0.9 & 1.0 & 1.1 & 1.2 \\
\hline Overall balance, cash b. (with grants) & 2.5 & -0.7 & -3.2 & 1.7 & 1.6 & 1.4 & 2.0 & 1.9 & 1.5 & 1.5 & 1.3 & 1.2 & 1.2 & 1.3 & 1.4 & 1.5 \\
\hline Primary balance & 8.1 & 6.0 & 5.7 & 5.1 & 4.7 & 4.1 & 3.9 & 3.6 & 3.0 & 2.8 & 2.4 & 2.2 & 2.0 & 2.0 & 1.9 & 1.9 \\
\hline Financing & & & & & & & & & & & & & & & & \\
\hline Net external & -0.8 & 1.9 & 5.9 & 1.1 & 1.5 & 1.4 & 1.2 & 0.7 & 0.9 & 0.6 & 0.6 & 0.1 & 0.0 & -0.1 & -0.4 & -0.5 \\
\hline Of which: drawings & 1.2 & 1.3 & 1.1 & 1.7 & 2.5 & 2.2 & 2.0 & 1.4 & 1.6 & 1.3 & 1.2 & 0.6 & 0.5 & 0.3 & 0.1 & 0.1 \\
\hline Net domestic & -1.6 & -1.2 & -2.3 & -2.8 & -3.1 & -2.8 & -3.2 & -2.6 & -2.4 & -2.2 & -1.9 & -1.3 & -1.2 & -1.2 & -1.0 & -1.0 \\
\hline Monetary survey & & & & & & & & & & & & & & & & \\
\hline Money supply & 16.5 & 17.5 & 19.5 & 19.6 & 19.9 & 20.3 & 20.6 & 20.8 & 21.1 & 21.3 & 21.5 & 21.8 & 22.0 & 22.2 & 22.5 & 22.7 \\
\hline Credit to the economy & 11.5 & 11.8 & 12.1 & 12.3 & 12.7 & 13.1 & 13.4 & 13.8 & 14.1 & 14.5 & 14.8 & 15.2 & 15.6 & 16.0 & 16.5 & 16.9 \\
\hline Debt & & & & & & & & & & & & & & & & \\
\hline External debt & & & & & & & & & & & & & & & & \\
\hline Outstanding & 73.1 & 65.4 & 57.6 & 51.0 & 46.5 & 42.6 & 39.1 & 35.5 & 32.6 & 29.8 & 27.3 & 24.5 & 22.2 & 20.0 & 17.8 & 15.8 \\
\hline Repayment of principal & 2.8 & 3.0 & 5.1 & 4.3 & 3.7 & 3.2 & 2.7 & 2.1 & 1.8 & 1.5 & 1.2 & 1.0 & 0.8 & 0.7 & 0.6 & 0.6 \\
\hline Payment of interest & 1.6 & 1.6 & 2.6 & 2.1 & 1.7 & 1.4 & 1.1 & 0.9 & 0.8 & 0.6 & 0.5 & 0.4 & 0.3 & 0.3 & 0.3 & 0.2 \\
\hline Debt service & 4.3 & 4.6 & 7.6 & 6.5 & 5.4 & 4.6 & 3.8 & 3.0 & 2.6 & 2.1 & 1.7 & 1.4 & 1.1 & 1.0 & 0.9 & 0.8 \\
\hline Debt servicing/exports & 13.8 & 15.5 & 27.3 & 23.5 & 20.6 & 18.4 & 15.4 & 12.1 & 10.4 & 8.5 & 6.8 & 5.6 & 4.3 & 3.8 & 3.3 & 3.0 \\
\hline Source: MINFI/DP & & & & & & & & & & & & & & & & \\
\hline
\end{tabular}




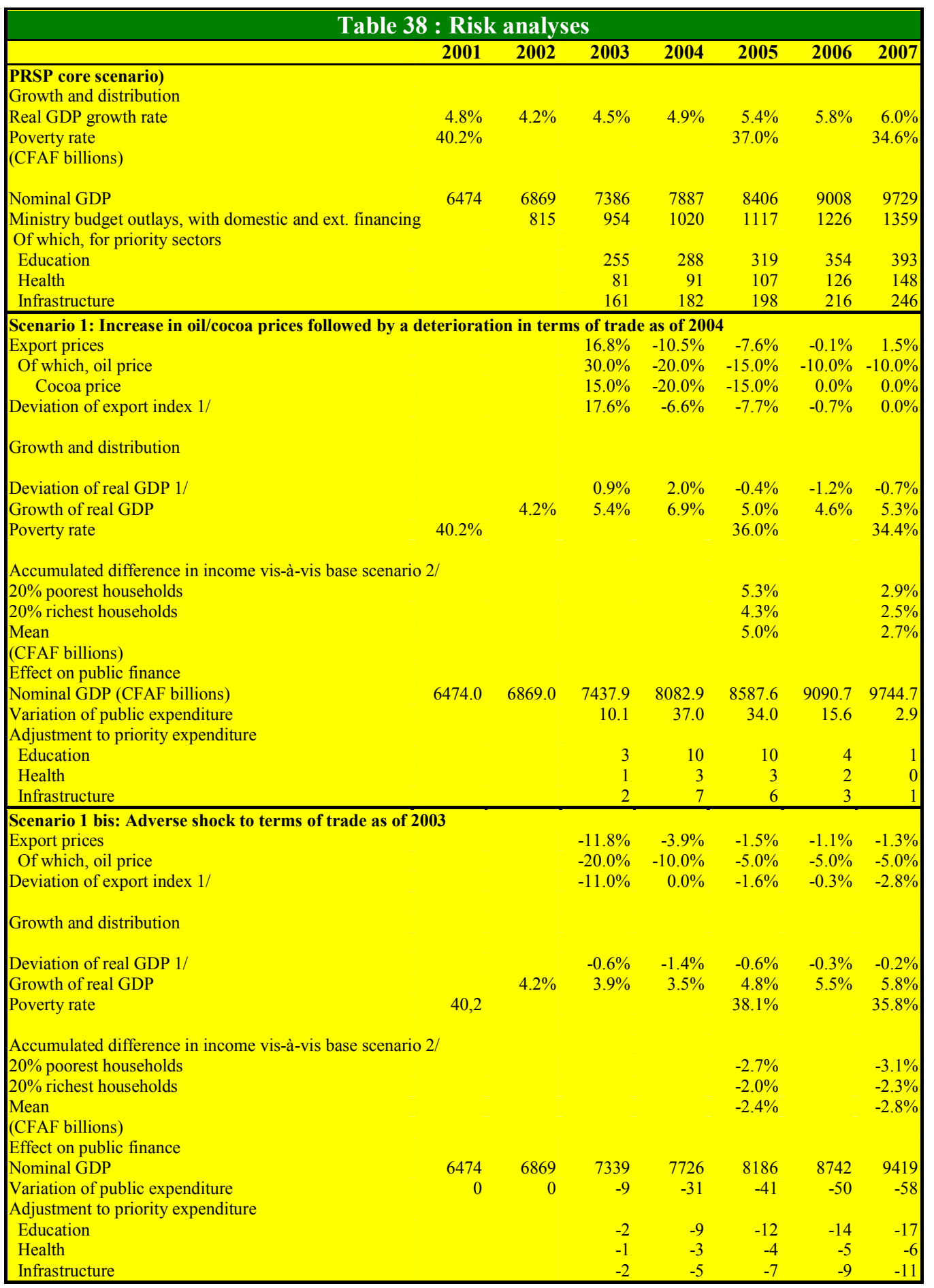




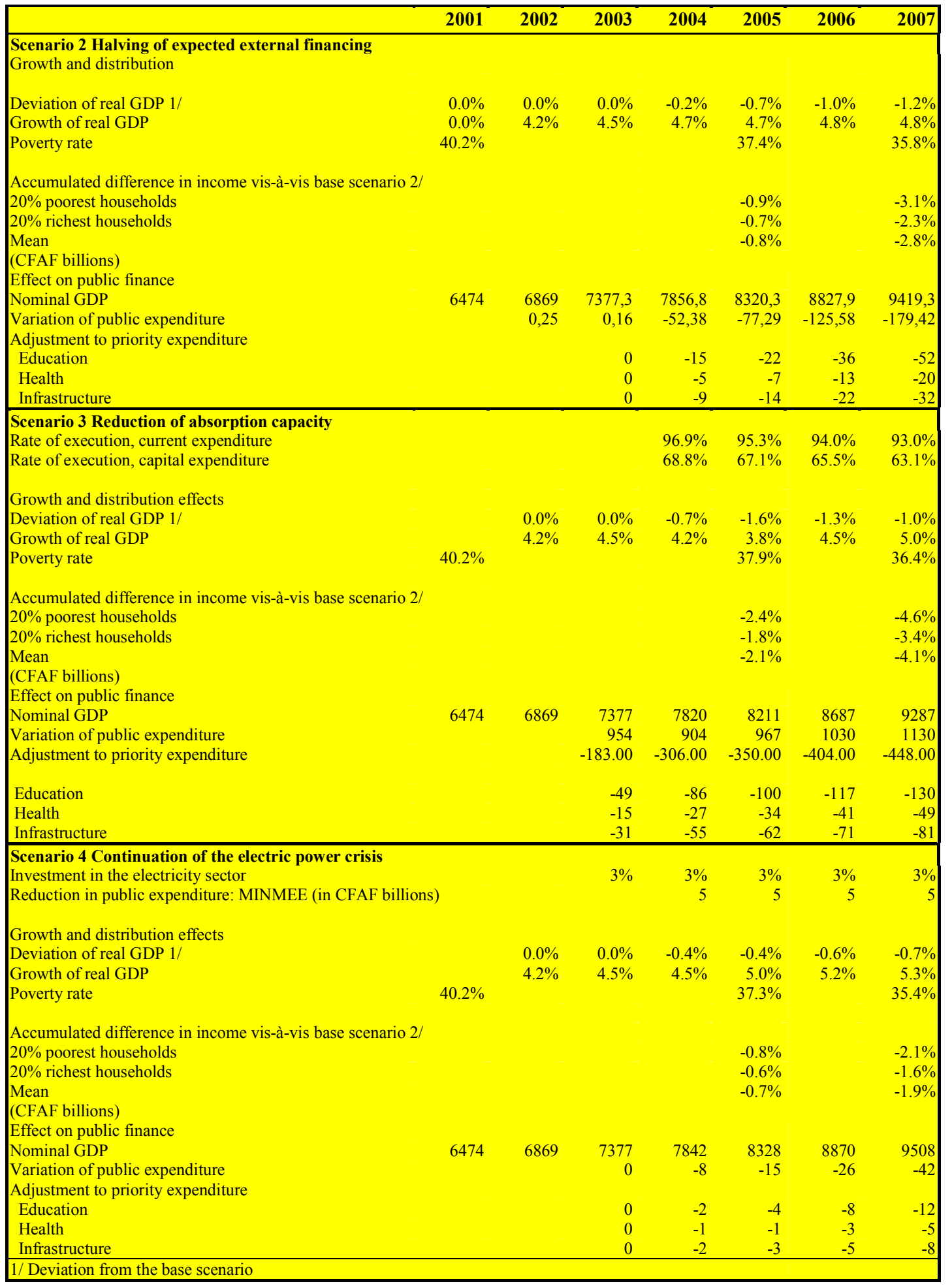


CHAPTER 5 : THE INSTITUTIONAL FRAMEWORK AND MECHANISMS FOR IMPLEMENTING AND MONITORING THE STRATEGY

CInternational Monetary Fund. Not for Redistribution 
Chapter 5: The Institutional framework and mechanisms for implementing and monitoring the strategy

\subsection{STEERING AND OVERSEEING IMPLEMENTATION OF THE STRATEGY}

482. The Cameroonian authorities consider that the system for steering and overseeing implementation of the strategy will be a key factor for its success. That system must be based on clear institutional arrangements and mechanisms tailored to match the multi-sector, multifaceted nature of the poverty reduction program.

483. Since satisfactory execution of the PRSP is one of the completion point requirements under the HIPC Initiative for Cameroon, supervision of implementation of the strategy through to completion point will fall to the Committee for Supervising and Coordinating Government Activities for Reaching Completion Point under the HIPC Initiative, established on October 28, 2002 by a decree of the Prime Minister and Head of Government, who chairs it. At the technical level, monitoring and evaluation activities will be coordinated by the Technical Committee for Monitoring Economic Programs (CTS), which will keep in close contact with all the agencies involved. The CTS will issue half-yearly reports on execution of the PRSP, based above all on data and/or analyses produced by the National Institute of Statistics, the ministries in each sector, and qualified nongovernmental entities.

484. The PRSP implementation reports will focus on the principal indicators selected (Cf. Annex 1) and develop gradually as reliable measurement of those indicators becomes possible. Once the periodic PRSP reports have been validated by the Interministerial Supervision Committee, they will be disseminated through channels that reach as many segments of the population as possible: the poor, in particular. Participatory evaluation of the outcomes will take place, above all, in the context of meetings between the authorities and civil society organizations designed not just to inform all the parties concerned but also to collect relevant observations and suggestions from all sides regarding monitoring of the strategy and to devise possible corrective measures when performance is unsatisfactory.

\subsection{MONITORING AND EVALUATION MECHANISMS}

485. The government intends to conduct the monitoring and evaluation of execution of its poverty reduction strategy in close coordination with all those involved. In that context, it will set up a reliable information mechanism to keep track of the situation and observe progress in combating this social blight. It will focus on three aspects: monitoring of execution, monitoring of impact, and monitoring of participation in the process.

486. The monitoring-of-execution component covers physical and financial implementation of all the actions, programs, and investment projects identified and included in the poverty reduction strategy. Monitoring will be conducted with a participatory approach involving central government and sectoral offices, local governments, the private sector, lenders and donors, and the beneficiaries.

487. In relation to the National Budget, monitoring of PRSP execution will be facilitated by the progress already made with installation of an integrated information system for managing public finance and the new classification of fiscal revenues and expenditure items. From now on, these two tools make it much easier to trace outlays according to their function. Particularly in the case of expenditure on poverty reduction, the Government intends to improve monitoring capabilities, from budget preparation, through the execution phase, to 
Chapter 5: The Institutional framework and mechanisms for implementing and monitoring the strategy

(external and internal) audits. Improvements will be developed along the main lines identified during the joint evaluation with the IMF and the World Bank.

488. The impact-monitoring component comprises analysis of poverty trends in monetary terms and with respect to the living conditions of the population. It will be carried out by (i) setting up a minimum statistical program (PSM) geared to specific objectives; (ii) monitoring relevant indicators that permit periodic analysis of changes in poverty; and (iii) gathering the data needed to produce the monitoring indicators.

489. Participatory monitoring will be carried out using appropriate methods. It will enable the authorities to hear what grassroots population groups think about the steps being taken to reduce poverty.

\subsection{IMPROVEMENTS IN THE PRODUCTION AND DISSEMINATION OF STATISTICAL INFORMATION}

490. In its concern (i) to keep track of the implementation of the actions it undertakes; (ii) to measure the outcomes and their impacts on target groups; and (iii) to take any necessary corrective measures, the government intends to establish appropriate statistical documentation facilities on poverty reduction. To that end, the focus will be on operations to collect data on household income and expenditure, on the supply, accessibility, and utilization of essential social services, especially education, health, and drinking water, and on nutrition, housing, and sanitation. In addition to these major items, further indicators will be constructed on vulnerable or disadvantaged groups, on gender issues, and on governance.

491. Producing these indicators presupposes a wide range of statistical operations. With that in mind, a minimum statistical program (PSM) was adopted by the National Statistics Council in May 2001. Apart from that program, targeted surveys will be conducted in specific fields and a strong effort will be made to tap administrative sources. The cost of setting up the PSM will be evaluated early in 2003 and reflected in the medium-term budget framework. The government will finance implementation of it out of National Budget resources, including part of the HIPC funds. It will also ask for technical and financial assistance from its development partners.

492. In 2003-2006, the authorities intend to carry out the following operations already contemplated in the PSM: the Third General Population and Housing Survey (RGPH), the employment survey, the informal sector survey, the survey on household consumption and living standards, the education survey, the Third Demographic and Health Survey (EDS III), with a special section on HIV/AIDS, the survey on the living environment in Yaoundé and in Douala, data gathering for the production of the second survey of social performance indicators in respect of women and children (TBS II), the survey to monitor public expenditure in the education and health sectors, known as the Public Expenditure Tracking Survey - PETS), the agricultural survey, and the nationwide 1.2.3 survey focusing especially on employment and the informal sector.

493. The authorities also envisage organizing targeted surveys in special areas for which information is lacking or which have not been explored, such as the environment, HIV/AIDS, vulnerable groups (children, the disabled, the elderly, etc.). They hope to make good use of administrative reports, which will make it possible to produce data at shorter intervals, above 
Chapter 5: The Institutional framework and mechanisms for implementing and monitoring the strategy

all with respect to statistics on activities in rural areas and in the basic social sectors, such as education, health, safe water supplies, electricity, etc. The authorities will see to it that these data are representative of the different regions and of other categories, such as gender. To that end, they intend to take steps to greatly enhance the quality of the information gathered, expand coverage, reduce time lags, and produce data on a regular basis.

494. The authorities also intend to ensure that the various data collecting activities and the sources of data are properly coordinated in order to ensure a coherent set of operations fully geared to poverty reduction. With that in mind, the schedule for carrying out the whole set of data gathering activities will take into account the indicators monitoring implementation of the first year of the PRSP, which is one of the requirements for completion point under the enhanced HIPC initiative.

495. The indicators generated will be disseminated through a wide range of channels and media to ensure that they reach the different categories of users. Apart from the traditional vehicles for disseminating and publishing statistical data (publications, seminars, the media, etc.), the following tools and channels will also be used: (i) Poverty Mapping, which will provide geographic visualization of all the poverty data and assist the planning and targeting of poverty reduction activities; (ii) the National Poverty Reduction Network, a forum for dialogue and consensus-building that will permit social supervision of all poverty reduction activities; and (iii) the General Data Dissemination System (GDDS), supported by the IMF.

496. With support from UNFPA, the government has also begun putting together economic and socio-demographic databases. In the course of 2003, with the help of the World Bank and France (through AFRISTAT), the government also intends to construct a web site at the National Institute of Statistics.

497. To carry out all these activities, the authorities are committed to improving the national statistical apparatus so that it can produce the required data on time. The National Statistics Institute was established already in April 2001 and steps have been taken to install its statutory bodies. The government will also seek to improve cooperation between the Institute and sectoral statistics departments.

498. To coordinate statistical activities, the government intends to reorganize the National Statistics Council so as to allow this body to perform its function as the body that sets strategic guidelines for statistical standardization, operational planning, and the allocation of resources. The idea is to structure it as a set of specialized committees with statutory members appointed for one or several terms.

\subsection{PARTICIPATORY MONITORING}

499. As in the preparation of the Poverty Reduction Strategy paper, the government will implement poverty reduction strategies and monitor and evaluate them in close coordination with all stakeholders. Thus, it intends to set up a National Poverty Reduction Network (RNRP), which will act above all as a forum for sharing experiences and exchanging data among groups and regarding participatory approaches, as well as a framework for societal supervision of all the activities undertaken to implement the poverty reduction strategy. 
Chapter 5: The Institutional framework and mechanisms for implementing and monitoring the strategy

500. With a view to establishing this mechanism, the government-with support from the UNDP, the GTZ, and the World Bank-organized a seminar in July 2001 to launch the preparation for such a network. The seminar identified the first features of the future network, namely how it would be structured, the links between its different parts, its management, steering, and orchestration mechanisms, its sources of financing, and its relations with the development stakeholder community. That meeting was attended by key figures in civil society, representatives of NGOs and of different religious confessions, university professors, trade union representatives, mayors' associations, and government officials.

501. As an instrument for coordinating and supporting poverty reduction activities, the RNRP will be open to all development players and facilitate a lasting partnership between civil society and the government. Through this network, the government expects to gain insight into the way grassroots segments of the population rate the effectiveness and efficiency of public spending on the social sectors and basic infrastructure, and to hear their suggestions for improving budget execution.

502. The authorities first want to test this Network in a pilot scheme before extending it to the whole of Cameroon. Considerable emphasis will be placed on communication.

503. The Committee for Consultations and Monitoring of HIPC Resources Management is one of the links in the nationwide participatory monitoring chain. Established by a decree of the Prime Minister, Head of Government in December 1, 2000, this committee is a consultative body responsible for ensuring proper use and equitable and optimal allocation of HIPC funds in combating poverty and enhancing governance. Thus, the Committee is above all responsible for (i) assessing the eligibility for HIPC financing of programs and projects proposed by the sectoral ministries, local governments, civil society organizations, and donors and creditors; (ii) issuing an opinion on the programs and projects to be financed out of HIPC funds that the government submits to Parliament for approval; (iii) proposing possible improvements to the HIPC-financed program; (iv) prescribing audits and/or periodic inspections of physical and financial project execution; and (v) making recommendations aimed at ensuring transparency and efficiency in the use of HIPC funds.

504. With its 19 members (7 representing the government, 5 representing bilateral and multilateral donors and creditors, and 7 representing civil society and the private sector), this Committee constitutes a highly innovative mechanism for preparing and adopting public expenditure projects and programs, and then monitoring management of part of the national budget. Although it was installed in June 2001, the Committee has not been fully operational, which explains the delay in executing the HIPC expenditures program. Significant operational improvements have taken place since November 2002, which have resulted in (i) the drafting and adopting of the Committee's internal rules of procedure, which define how it operates; (ii) adoption of a manual of procedures for determining the methodology and criteria to be used in preparing and selecting projects eligible for HIPC financing; and (iii) the approval of a second set of projects in November 2002, following on those approved in October and December 2001.

\subsection{CAPACITY-BUILDING}

505. The government intends, in the execution stage of the program, to continue and step up the participatory process launched in January 2000, with a view to preparing the interim 
Chapter 5: The Institutional framework and mechanisms for implementing and monitoring the strategy

poverty reduction strategy paper. It then became apparent that it was important to enhance the skills of the different actors, to enable them to make an accurate assessment of the situation and articulate corrective measures. This capacity building is a sine qua non for endowing the participatory monitoring and evaluation system with the tools it needs to be fully effective.

506. The government will call upon its partners for development for the technical and financial support needed to undertake well-targeted activities to boost the ability of local and central government officials, as well as that of organized groups in civil society to perform participatory monitoring and evaluation tasks effectively. In this context, a robust information and awareness campaign will get under way in 2003 to familiarize all stakeholders with the indicators chosen and how they develop.

507. As regards statistical monitoring of implementation of the PRSP and of progress made in poverty reduction, the authorities are expecting to strengthen coordination of the activities carried out by the national statistical system and the capacity of the Institute to produce the data it is responsible for. Sectoral statistical departments will also be strengthened so that they can improve both the quality and regularity of the data they produce.

508. In its concern to facilitate the involvement of private sector and civil society players in the participatory process of implementing, monitoring, and evaluating the actions envisaged in the PRSP, the government intends to organize seminar-workshops to boost skills at different levels. These activities will take place in the framework of the National Poverty Reduction Network. 
ANNEXES 
Annex : Implementation matrix

\section{ANNEX 1 IMPLEMENTATION MATRIX}

STRATEGY AND MEASURES FOR REDUCTION OF POVERTY

\begin{tabular}{|c|c|c|c|c|c|}
\hline Strategy & $\begin{array}{l}\text { Field/ } \\
\text { policy }\end{array}$ & Actions/measures & Performance indicators & Timetable & $\begin{array}{l}\text { Assigned } \\
\text { responsibility }\end{array}$ \\
\hline \multicolumn{6}{|c|}{ Axis 1: Promotion of a stable macroeconomic framework } \\
\hline & \multicolumn{5}{|c|}{ 1.1 Macroeconomic and budgetary framework } \\
\hline & \multirow{3}{*}{ 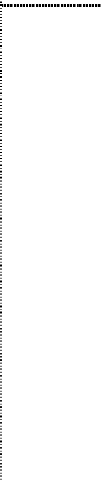 } & $\begin{array}{l}\text { Maintain a stable macroeconomic framework and implement in satisfactory fashion } \\
\text { the final year of the second three-year economic and financial program under FRPC }\end{array}$ & $\begin{array}{l}\text { Satisfactory execution of the third year of the FRPC } \\
\text { program. }\end{array}$ & 2003 & $\begin{array}{l}\text { MINFI } \\
\text { MINEPAT }\end{array}$ \\
\hline & & $\begin{array}{l}\text { Use the budget savings from the debt reduction in line with the criteria provided in } \\
\text { the decision document (control and monitoring mechanisms) }\end{array}$ & $\begin{array}{l}\text { Satisfactory execution of projects deemed acceptable by } \\
\text { the CCS/PPTE and approved by the government. }\end{array}$ & 2003 & $\begin{array}{l}\text { MINFI } \\
\text { MINEPAT } \\
\text { CCS/PPTE }\end{array}$ \\
\hline & & $\begin{array}{l}\text { Execute in satisfactory manner the Third Structural Adjustment Credit (CAS III); } \\
\text { observe the closing date of the Credit: June } 30,2003\end{array}$ & $\begin{array}{l}\text { Conditionality for withdrawals from the floating tranche } \\
\text { intended for port facility renovations implemented; } \\
\text { Formulation of the privatization of banana, hevea and } \\
\text { palm oil production (CDC); } \\
\text { Preparation of pertinent competitive bidding documents; } \\
\text { Preparation of the CAMTEL preliminary business plan } \\
\text { and specifications; } \\
\text { Finalization of the transaction documents for } \\
\text { transforming SNEC into a concession. }\end{array}$ & 2003 & $\begin{array}{l}\text { MINFI } \\
\text { MINEPAT }\end{array}$ \\
\hline & \multicolumn{5}{|c|}{1.2 Pursue the implementation of the action plan for improving the management of public expenditure } \\
\hline & & Continue implementation of the SIGEFI & $\begin{array}{l}\text { Installed interfaces; } \\
\text { Timely production of summary tables on the execution of } \\
\text { the government budget. }\end{array}$ & 2003 & $\begin{array}{l}\text { MINFI } \\
\text { MINEPAT }\end{array}$ \\
\hline & & Improve the quality of public investment outlays & $\begin{array}{l}\text { Formulation of quality criteria for expenditures; } \\
\text { Production of periodic reports on physical and financial } \\
\text { performance of the budgets. }\end{array}$ & Ongoing & MINEPAT \\
\hline & & Ensure exhaustive and reliable production of Treasury account balances & Regular production of reliable balances. & Ongoing & MINFI \\
\hline & & Systematize audits of public expenditure & $\begin{array}{l}\text { Methodology and procedures of audits determined and } \\
\text { published; } \\
\text { Regular publication of audit reports. }\end{array}$ & Ongoing & $\begin{array}{l}\text { MINFI } \\
\text { MINEPAT }\end{array}$ \\
\hline & & Continue formulation of targeted budgets & $\begin{array}{l}\text { Targeted budgets drawn up and submitted to the National } \\
\text { Assembly. }\end{array}$ & $\begin{array}{l}\text { Starting in } \\
2005\end{array}$ & \\
\hline & & Pursue a prudent policy of indebtedness favoring borrowing at concessional rates & $\begin{array}{l}\text { Degree of concessionality of borrowing approved by the } \\
\text { government. }\end{array}$ & Ongoing & $\begin{array}{l}\text { MINFI } \\
\text { MINEPAT } \\
\text { CAA }\end{array}$ \\
\hline
\end{tabular}


Annex : Implementation matrix

STRATEGY AND MEASURES FOR REDUCTION OF POVERTY

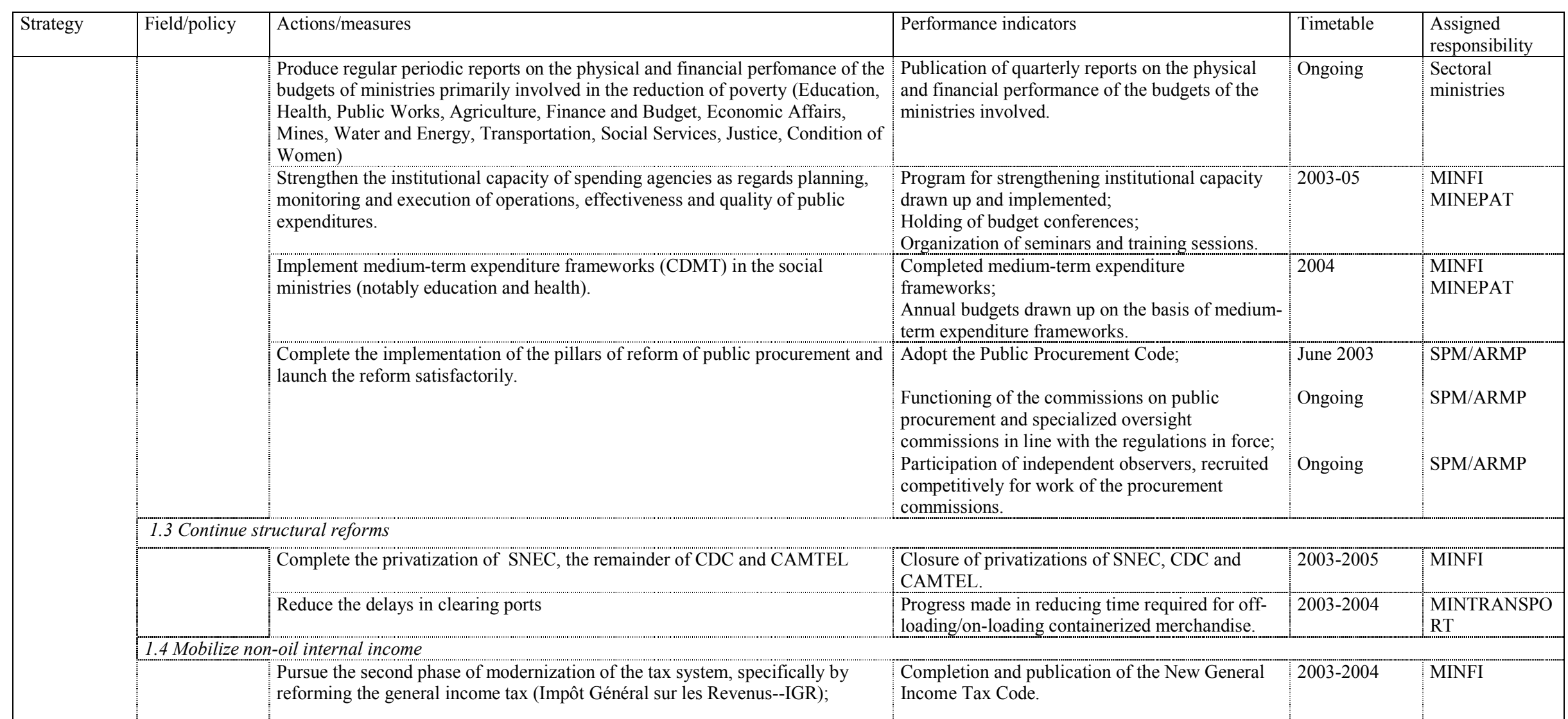


Annex : Implementation matrix

STRATEGY AND MEASURES FOR REDUCTION OF POVERTY

\begin{tabular}{|c|c|c|c|c|c|}
\hline \multirow[t]{2}{*}{ Strategy } & Field/policy & Actions/measures & Performance indicators & Timetable & $\begin{array}{l}\text { Assigned } \\
\text { responsibility }\end{array}$ \\
\hline & & $\begin{array}{l}\text { Continue to implement the program for securing customs revenue, specifically } \\
\text { by securing and limiting exemptions from customs duties. }\end{array}$ & $\begin{array}{l}\text { Strengthening of security; } \\
\text { Publication of the limited list of exemptions; } \\
\text { Monitoring of compliance with the exceptional } \\
\text { exemptions list. }\end{array}$ & $\begin{array}{l}\text { Starting in } \\
2003\end{array}$ & MINFI \\
\hline \multicolumn{6}{|c|}{ Axis 2: Strengthening growth through diversification of the economy } \\
\hline & \multicolumn{5}{|c|}{2.1 Development of the rural sector } \\
\hline & & $\begin{array}{l}\text { Improve peasant access to modern, high-yield farming techniques by } \\
\text { disseminating widely results of agronomic research }\end{array}$ & $\begin{array}{l}\text { Identification of research results; } \\
\text { Popularization and publication of research results; } \\
\text { Number or peasant organizations having accessed } \\
\text { the results of agronomic research. }\end{array}$ & Ongoing & $\begin{array}{l}\text { MINAGRI } \\
\text { MINEPIA } \\
\text { MINEF } \\
\text { MINREST }\end{array}$ \\
\hline & & Promote community development & Implementation of the PNDP and the PADC. & Ongoing & $\begin{array}{l}\text { MINAGRI } \\
\text { MINEPIA } \\
\text { MINEF }\end{array}$ \\
\hline & & $\begin{array}{l}\text { Promote and support the development of farmers' associations and other } \\
\text { organizations linked to farming }\end{array}$ & $\begin{array}{l}\text { Development of a concerted strategy for } \\
\text { promoting and developing peasant organizations; } \\
\text { Number of organizations supported. }\end{array}$ & Ongoing & $\begin{array}{l}\text { MINAGRI } \\
\text { MINEPIA } \\
\text { MINEF } \\
\text { MINAT }\end{array}$ \\
\hline & & $\begin{array}{l}\text { Launch specific high-priority programs of rural support for development and } \\
\text { organization of priority sectors }\end{array}$ & Start-up of specific programs. & 2003 & MINAGRI \\
\hline & & Get the Forestry/Environment Sectoral Program (PSFE) under way & Effective operation of the (PSFE) & 2003 & MINEF \\
\hline & & Finalize and implement the law on the equalization fund & Finalized, published, implemented law. & $2003-2004$ & $\begin{array}{l}\text { MINEF } \\
\text { MINAT } \\
\text { MINFI }\end{array}$ \\
\hline & & Draw up and implement a master plan for rural water supply & $\begin{array}{l}\text { Completion and publication of the master plan for } \\
\text { rural water supply. }\end{array}$ & 2004 & $\begin{array}{l}\text { MINAGRI } \\
\text { MINEPIA } \\
\text { MINMEE }\end{array}$ \\
\hline & & Enforce the law on community forests & The law voted on, published and implemented. & $2003-2004$ & MINEF \\
\hline
\end{tabular}


Annex : Implementation matrix

STRATEGY AND MEASURES FOR REDUCTION OF POVERTY

\begin{tabular}{|c|c|c|c|c|c|}
\hline Strategy & Field/policy & Actions/measures & Performance indicators & Timetable & $\begin{array}{l}\text { Assigned } \\
\text { responsibility }\end{array}$ \\
\hline & & Regulate the use of non-ligneous forest products & Published, promulgated law & $2003-2004$ & MINEF \\
\hline & & $\begin{array}{l}\text { Improve the productivity of the fishing industry by introducing new species of } \\
\text { hatchlings }\end{array}$ & Number of hatchlings introduced & $2003-2005$ & MINEPIA \\
\hline & \multicolumn{2}{|c|}{2.2 Development of the industrial sector } & & & \\
\hline & & Finalize the sectoral strategy for industrial development & Finalization and publication of the strategy & 2003 & MINDIC \\
\hline & & $\begin{array}{l}\text { Undertake a diagnostic study of the industrial sector to identify and select the } \\
\text { segments that have significant growth potential }\end{array}$ & $\begin{array}{l}\text { Publication of the report on the study; } \\
\text { Identification of the high-potential segments. }\end{array}$ & 2004 & MINDIC \\
\hline & & Draw up a specific policy for promoting local subcontracting & $\begin{array}{l}\text { Publication of a policy document on promotion of } \\
\text { subcontracting. }\end{array}$ & $2004-2005$ & \\
\hline & & $\begin{array}{l}\text { Establish mechanisms that promote dialogue between research institutes, } \\
\text { universities, university-level business education institutions and businesses, for } \\
\text { the purpose of disseminating the results of research and development activities }\end{array}$ & $\begin{array}{l}\text { Creation of mechanisms that promote dialogue; } \\
\text { Use of the research results by Cameroonian } \\
\text { businesses. }\end{array}$ & $2004-2005$ & $\begin{array}{l}\text { MINDIC } \\
\text { MINETFOP } \\
\text { MINESUP }\end{array}$ \\
\hline & & Implement the wood products industry industrialization plan & Implementation of the industrialization plan & $\begin{array}{l}\text { Starting in } \\
2003\end{array}$ & MINDIC \\
\hline & & $\begin{array}{l}\text { Promote the creation of information and communications service and } \\
\text { technology industries. }\end{array}$ & $\begin{array}{l}\text { Development and implementation of a concerted } \\
\text { strategy }\end{array}$ & $\begin{array}{l}\text { Starting in } \\
2003\end{array}$ & MINDIC \\
\hline & & $\begin{array}{l}\text { Promote the creation of second- and third-level transformation in the wood } \\
\text { products industry }\end{array}$ & $\begin{array}{l}\text { Development and implementation of a concerted } \\
\text { strategy for creating enterprises; } \\
\text { Initiatives for creating enterprises in this industry. }\end{array}$ & $\begin{array}{l}\text { Starting in } \\
2003\end{array}$ & MINDIC \\
\hline & & Promote standardization and quality control & $\begin{array}{l}\text { Publication and enforcement of standardization } \\
\text { regulations; } \\
\text { Number of standards adopted; } \\
\text { Completed mechanisms for monitoring standards } \\
\text { and compliance agents trained and equipped. }\end{array}$ & $2003-2004$ & MINDIC \\
\hline & & Adopt a Development Policy Declaration for SMEs/SMIs & $\begin{array}{l}\text { Development and implementation of a concerted } \\
\text { strategy for promoting SMEs/SMIs. }\end{array}$ & $2003-2005$ & MINDIC \\
\hline
\end{tabular}


Annex : Implementation matrix

STRATEGY AND MEASURES FOR REDUCTION OF POVERTY

\begin{tabular}{|c|c|c|c|c|c|}
\hline Strategy & Field/policy & Actions/measures & Performance indicators & Timetable & $\begin{array}{l}\text { Assigned } \\
\text { responsibility }\end{array}$ \\
\hline & \multicolumn{5}{|c|}{ 2.3 Development of tourism,cultural services and support services for the productive sector } \\
\hline & \multicolumn{2}{|c|}{ Promotion of the tourism and cultural services sector } & & & \\
\hline & \multirow[t]{7}{*}{ (1) } & Finalize the sectoral strategy for tourism & $\begin{array}{l}\text { Finalization, publication, dissemination and } \\
\text { implementation of a concerted strategy for } \\
\text { development of tourism; } \\
\text { Incentives for creating tourism enterprises } \\
\text { identified and implemented. }\end{array}$ & 2003 & MINTOUR \\
\hline & & $\begin{array}{l}\text { Organize in Cameroon's } 10 \text { provinces awareness seminars to encourage } \\
\text { young graduates to enter the tourism industry }\end{array}$ & $\begin{array}{l}\text { Organization of awareness seminars in the } \\
\text { provinces. }\end{array}$ & $2003-2005$ & MINTOUR \\
\hline & & Provide standards for institutions and agencies involved in tourism & $\begin{array}{l}\text { Number of institutions and agencies provided } \\
\text { with standards. }\end{array}$ & $2004-2005$ & MINTOUR \\
\hline & & $\begin{array}{l}\text { Undertake a general inventory of Cameroon's material and spiritual } \\
\text { patrimony }\end{array}$ & Publication of a report on the complete inventory. & $2003-2004$ & MINCULT \\
\hline & & $\begin{array}{l}\text { Define and implement a strategy for developing the cinema industry and } \\
\text { audiovisual products }\end{array}$ & $\begin{array}{l}\text { Development and implementation of a concerted } \\
\text { strategy for developing the cinema industry. }\end{array}$ & $2003-2004$ & $\begin{array}{l}\text { MINCULT } \\
\text { MINCOM }\end{array}$ \\
\hline & & Create the National Museum of Cameroon & Completed and equipped museum. & $2003-2005$ & $\begin{array}{l}\text { MINCULT } \\
\text { MINTOUR } \\
\text { UNESCO }\end{array}$ \\
\hline & & Create a National Arts and Culture Institute & $\begin{array}{l}\text { Completed and operating Institut National des } \\
\text { Arts et de la Culture. }\end{array}$ & $2003-2005$ & $\begin{array}{l}\text { MINCULT } \\
\text { MINEDUC } \\
\text { UNESCO }\end{array}$ \\
\hline & \multicolumn{2}{|c|}{ Promotion of financial intermediation } & & & \\
\hline & & Make Cameroon's financial market operational (Douala Stock Exchange) & Effective operational securities market. & 2003 & MINFI \\
\hline & & $\begin{array}{l}\text { Continue to restore health to the sector of savings and loan cooperatives } \\
\text { (COOPEC) }\end{array}$ & $\begin{array}{l}\text { Existing COOPECs in compliance with the } \\
\text { regulations in force. }\end{array}$ & $\begin{array}{l}\text { Starting in } \\
2003\end{array}$ & $\begin{array}{l}\text { MINFI } \\
\text { PPMF } \\
\text { COBAC }\end{array}$ \\
\hline
\end{tabular}


Annex : Implementation matrix

STRATEGY AND MEASURES FOR REDUCTION OF POVERTY

\begin{tabular}{|c|c|c|c|c|c|}
\hline Strategy & Field/policy & Actions/measures & Performance indicators & Timetable & $\begin{array}{l}\text { Assigned } \\
\text { responsibility }\end{array}$ \\
\hline & & $\begin{array}{l}\text { Promote relations between the MFIs and commercial banks for the purpose } \\
\text { of introducing micro-lending into their loan portfolios }\end{array}$ & $\begin{array}{l}\text { Development and implementation of a concerted } \\
\text { strategy for promoting relations between the MFIs } \\
\text { and commercial banks. }\end{array}$ & $2003-2005$ & $\begin{array}{l}\text { MINFI } \\
\text { MINAGRI }\end{array}$ \\
\hline & & Provide incentives to broaden the country's banking coverage & $\begin{array}{l}\text { Development and implementation of a concerted } \\
\text { strategy for developing the banking sector. }\end{array}$ & $2003-2005$ & $\begin{array}{l}\text { MINFI, } \\
\text { APECCAM }\end{array}$ \\
\hline & & $\begin{array}{l}\text { Promote the development of financing institutions and financing } \\
\text { mechanisms geared to the rural sector }\end{array}$ & $\begin{array}{l}\text { Development and implementation of a concerted } \\
\text { strategy for developing appropriate financing } \\
\text { institutions and mechanisms; } \\
\text { Identification and implementation of incentives to } \\
\text { promote the appropriate institutions and measures. }\end{array}$ & $2003-2005$ & MINAGRI \\
\hline & & Promote decentralized financing systems & $\begin{array}{l}\text { Development and implementation of a concerted } \\
\text { strategy for developing appropriate financing } \\
\text { institutions and mechanisms; } \\
\text { Identification and implementation of incentives to } \\
\text { promote the appropriate institutions and measures. }\end{array}$ & $2003-2005$ & $\begin{array}{l}\text { MINAGRI } \\
\text { MINFI }\end{array}$ \\
\hline & & Promote the mobilization of savings & $\begin{array}{l}\text { Development and implementation of a concerted } \\
\text { strategy for mobilization of savings; } \\
\text { Identification and implementation of incentives to } \\
\text { mobilize savings. }\end{array}$ & $2003-2005$ & $\begin{array}{l}\text { MINAGRI } \\
\text { MINFI }\end{array}$ \\
\hline \multicolumn{6}{|c|}{ Axis 3: Energizing the private sector } \\
\hline & & Continue the fight against dumping and contraband & $\begin{array}{l}\text { Measures for fighting dumping contraband } \\
\text { identified and implemented; } \\
\text { Monitoring agents trained and equipped. }\end{array}$ & Ongoing & $\begin{array}{l}\text { MINDIC } \\
\text { MINFI }\end{array}$ \\
\hline & & $\begin{array}{l}\text { Complete major infrastructure construction projects supporting the } \\
\text { industrial sector (bridge over the Wouri; Douala and Yaoundé street } \\
\text { systems; major highway segments linking the country's grid) }\end{array}$ & $\begin{array}{l}\text { Identification of priority projects in collaboration } \\
\text { with the private sector; } \\
\text { Mobilization of funding and completion of } \\
\text { construction projects. }\end{array}$ & $\begin{array}{l}\text { Starting in } \\
2003\end{array}$ & MINDIC \\
\hline
\end{tabular}


Annex : Implementation matrix

STRATEGY AND MEASURES FOR REDUCTION OF POVERTY

\begin{tabular}{|c|c|c|c|c|c|}
\hline Strategy & Field/policy & Actions/measures & Performance indicators & Timetable & $\begin{array}{l}\text { Assigned } \\
\text { responsibility }\end{array}$ \\
\hline & & $\begin{array}{l}\text { Encourage the creation of businesses involved in industrial maintenance } \\
\text { and repairs. }\end{array}$ & $\begin{array}{l}\text { Development and implementation of a concerted } \\
\text { strategy for creating businesses involved in } \\
\text { industrial maintenance and repairs; } \\
\text { Identification and implementation of incentives to } \\
\text { create businesses involved in industrial maintenance } \\
\text { and repairs. }\end{array}$ & $\begin{array}{l}\text { Starting in } \\
2003\end{array}$ & MINDIC \\
\hline & & $\begin{array}{l}\text { Promote the generic drugs industry and the agricultural tool and equipment } \\
\text { manufacturing industry }\end{array}$ & $\begin{array}{l}\text { Development and implementation of a concerted } \\
\text { strategy for promotion of the generic drugs industry } \\
\text { and the agricultural tool and equipment } \\
\text { manufacturing industry; } \\
\text { Incentives identified and implemented. }\end{array}$ & $\begin{array}{l}\text { Starting in } \\
2003\end{array}$ & MINDIC \\
\hline & & $\begin{array}{l}\text { Create a business incubator program in the subsectors of SME/SMI and the } \\
\text { crafts }\end{array}$ & $\begin{array}{l}\text { Development and implementation of a program of } \\
\text { business incubators }\end{array}$ & $\begin{array}{l}\text { Starting in } \\
2003\end{array}$ & MINDIC \\
\hline & & $\begin{array}{l}\text { Establish the instruments and institutions called for in the Investment } \\
\text { Charter: specifically, the Council on Regulation of Competitiveness, the } \\
\text { Agency for the Promotion of Investment and Exports, and the Monitor of } \\
\text { Industry and Commerce } \\
\text { Increase the involvement of the private sector in (i) the development and } \\
\text { implementation of technical and vocational training programs; (ii) the } \\
\text { development of programs in applied research; (iii) the funding of programs } \\
\text { to popularize computer science }\end{array}$ & $\begin{array}{l}\text { Effective creation of the institutions called for in the } \\
\text { Investment Charter } \\
\text { Signing of partnership agreements between the } \\
\text { private sector and the applied research training } \\
\text { institutions }\end{array}$ & 2004 & MINDIC \\
\hline & & $\begin{array}{l}\text { Promote the strengthening of abilities of private sector organizations to } \\
\text { help them participate in the design an implementation of economic policies } \\
\text { and to improve effectively the services rendered by their members }\end{array}$ & $\begin{array}{l}\text { Creation of a concerted program to strengthen the } \\
\text { abilities of the private sector; } \\
\text { Number of seminars and workshops organized to } \\
\text { effect this strengthening. }\end{array}$ & $2003-2004$ & MINDIC \\
\hline
\end{tabular}


Annex : Implementation matrix

STRATEGY AND MEASURES FOR REDUCTION OF POVERTY

\begin{tabular}{|c|c|c|c|c|c|}
\hline Strategy & Field/policy & Actions/measures & Performance indicators & Timetable & $\begin{array}{l}\text { Assigned } \\
\text { responsibility }\end{array}$ \\
\hline & & Eliminate factors impeding development of the private sector & $\begin{array}{l}\text { Identification of the impeding factors; } \\
\text { Development of measures for eliminating } \\
\text { impediments to development, in collaboration with } \\
\text { the private sector; } \\
\text { Publication of these measures as a way of } \\
\text { eliminating impediments to the development of the } \\
\text { private sector. }\end{array}$ & $2003-2005$ & MINDIC \\
\hline \multicolumn{6}{|c|}{ Axis 4: Development of economic infrastructures and natural resources } \\
\hline & \multicolumn{5}{|c|}{ 4.1 Development of basic infrastructures } \\
\hline & & $\begin{array}{l}\text { Increase resources devoted to renewal and maintenance of the priority road } \\
\text { network }\end{array}$ & $\begin{array}{l}\text { Portion of the annual budget allocated to capital } \\
\text { investments; } \\
\text { Amount of annual funding allocated to the Roads } \\
\text { Fund. }\end{array}$ & 2003 & $\begin{array}{l}\text { MINTP } \\
\text { MINEPAT } \\
\text { MINFI }\end{array}$ \\
\hline & & $\begin{array}{l}\text { Strengthen expertise in planning road maintenance work and the pertinent } \\
\text { procurement process }\end{array}$ & Program drawn up and implemented. & $2003-2004$ & MINTP \\
\hline & & $\begin{array}{l}\text { Promote the use of highly labor-intensive methods in rural road repair } \\
\text { work and in repairs of secondary roads }\end{array}$ & $\begin{array}{l}\text { Adoption of a concerted strategy for promotion of } \\
\text { highly labor-intensive methods (HIMO). }\end{array}$ & 2003 & $\begin{array}{l}\text { MINTP } \\
\text { MINUH/ } \\
\text { MINVILLE } \\
\text { TOWNS }\end{array}$ \\
\hline & & Finalize the strategy for river and lake development & $\begin{array}{l}\text { Adoption, publication and dissemination of the } \\
\text { strategy }\end{array}$ & 2004 & $\begin{array}{l}\text { MINTRANSPO } \\
\text { RT }\end{array}$ \\
\hline & & Finalize the strategy for developing transportation equipment and services & $\begin{array}{l}\text { Adoption, publication and dissemination of the } \\
\text { strategy }\end{array}$ & 2004 & $\begin{array}{l}\text { MINTRANSPO } \\
\text { RT }\end{array}$ \\
\hline & \multicolumn{2}{|c|}{$\begin{array}{l}4.2 \text { Development of telecommunications and the technologies of information and } \\
\text { communications }\end{array}$} & & & \\
\hline & & $\begin{array}{l}\text { Finalize the sectoral strategy of the Ministry of Post Office and } \\
\text { Telecommunications }\end{array}$ & $\begin{array}{l}\text { Adoption, publication and dissemination of the } \\
\text { strategy }\end{array}$ & 2003 & MINPOSTEL \\
\hline & & $\begin{array}{l}\text { Facilitate the development of technologies of information and } \\
\text { communications }\end{array}$ & $\begin{array}{l}\text { Creation of the appropriate instituutional and } \\
\text { regulatory framework. }\end{array}$ & $\begin{array}{l}\text { Starting in } \\
2003\end{array}$ & $\begin{array}{l}\text { MINCOM } \\
\text { MINPOSTEL }\end{array}$ \\
\hline & & Open multimedia community centers in the ten provinces & At least ten centers are operational & $\begin{array}{l}\text { Starting in } \\
2003\end{array}$ & MINCOM \\
\hline
\end{tabular}


Annex : Implementation matrix

STRATEGY AND MEASURES FOR REDUCTION OF POVERTY

\begin{tabular}{|c|c|c|c|c|c|}
\hline Strategy & Field/policy & Actions/measures & Performance indicators & Timetable & $\begin{array}{l}\text { Assigned } \\
\text { responsibility }\end{array}$ \\
\hline & & $\begin{array}{l}\text { Extend the fiber optic line installed along the Doba-Kribi pipeline up to } \\
\text { Douala and Bafoussam, thereby creating a high speed triangle with lower } \\
\text { communications transaction costs for individual users }\end{array}$ & $\begin{array}{l}\text { Effective extension of the installed fiber optic line to } \\
\text { Douala and Bafoussam; } \\
\text { Development and implementation of a policy for } \\
\text { marketing the use of the fibre optic system. }\end{array}$ & $2003-2004$ & $\begin{array}{l}\text { MINPOSTEL } \\
\text { SNH } \\
\text { COTCO }\end{array}$ \\
\hline & & Install the cryptology and certification systems & Effective implementation of the cryptology system & $2003-2005$ & $\begin{array}{l}\text { MINPOSTEL } \\
\text { OPERATEURS }\end{array}$ \\
\hline & & Create the intergovernmental communications network & Amount of additional investments in this sector & $2003-2005$ & MINPOSTEL \\
\hline & & Create a legal and regulatory framework suited to the TICs & Implementation of the legal framework & $2003-2004$ & $\begin{array}{l}\text { MINPOSTEL } \\
\text { MINJUSTICE }\end{array}$ \\
\hline & & $\begin{array}{l}\text { Improve the capacity and quality of telecommunications infrastructures } \\
\text { (multiplication of fixed and mobile telephone lines) }\end{array}$ & $\begin{array}{l}\text { Number of installations performed; } \\
\text { Broadening of the service coverage using fixed and } \\
\text { mobile telephone facilities. }\end{array}$ & $2003-2005$ & $\begin{array}{l}\text { MINPOSTEL } \\
\text { OPERATEURS }\end{array}$ \\
\hline & & Develop the telemedicine project & Development and adoption of the project & $2003-2004$ & $\begin{array}{l}\text { MINPOSTEL } \\
\text { MINSANTE }\end{array}$ \\
\hline & & Install new rural radios and create community telecenters in rural areas & $\begin{array}{l}\text { Number of new radios installed; } \\
\text { Number of telecenters created. }\end{array}$ & $\begin{array}{l}\text { Starting in } \\
2003\end{array}$ & $\begin{array}{l}\text { MINCOM } \\
\text { MINPOSTEL }\end{array}$ \\
\hline & & $\begin{array}{l}\text { Update organization and programs of study in general technical secondary } \\
\text { education and in vocational training by introducing the teaching of } \\
\text { computer science }\end{array}$ & $\begin{array}{l}\text { Adoption, publication and dissemination of a new } \\
\text { program; } \\
\text { Computer hardware available. }\end{array}$ & $\begin{array}{l}\text { Starting in } \\
2003\end{array}$ & $\begin{array}{l}\text { MINEDUC } \\
\text { MINETFOP }\end{array}$ \\
\hline & 4.3 Managen & natural resources & & & \\
\hline & Promote acc & drinking water & & & \\
\hline & & Draw up a sectoral water strategy & $\begin{array}{l}\text { Adoption of a concerted strategy, publication and } \\
\text { dissemination }\end{array}$ & 2003 & MINMEE \\
\hline
\end{tabular}


Annex : Implementation matrix

STRATEGY AND MEASURES FOR REDUCTION OF POVERTY

\begin{tabular}{|c|c|c|c|c|c|}
\hline Strategy & Field/policy & Actions/measures & Performance indicators & Timetable & $\begin{array}{l}\text { Assigned } \\
\text { responsibility }\end{array}$ \\
\hline & & $\begin{array}{l}\text { Step up water provision programs (wells, controlled springs, drilling, mini- } \\
\text { systems of water conveyance) }\end{array}$ & Number of projects completed & $\begin{array}{l}\text { Starting in } \\
2003\end{array}$ & $\begin{array}{l}\text { MINMEE } \\
\text { MINAGRI }\end{array}$ \\
\hline & \multicolumn{5}{|c|}{ Develop and promote industrial mining projects (mines and hydrocarbon extraction) } \\
\hline & \multicolumn{2}{|c|}{ Design a Cameroonian mineral plan } & $\begin{array}{l}\text { Adoption, publication and dissemination of the } \\
\text { mining plan }\end{array}$ & 2004 & $\begin{array}{l}\text { MINMEE } \\
\text { Economic } \\
\text { operators }\end{array}$ \\
\hline & & Strengthen institutional capacities & $\begin{array}{l}\text { Development of a program for strengthening these } \\
\text { capacities; } \\
\text { Training of agents working in the new organizations }\end{array}$ & $2003-2004$ & $\begin{array}{l}\text { MINMEE } \\
\text { Economic } \\
\text { operators }\end{array}$ \\
\hline & & $\begin{array}{l}\text { Support the production, transformation and marketing of products of } \\
\text { artisanal mines }\end{array}$ & Publication of measures taken & $\begin{array}{l}\text { Starting in } \\
2003\end{array}$ & $\begin{array}{l}\text { MINMEE } \\
\text { Economic } \\
\text { operators }\end{array}$ \\
\hline & & $\begin{array}{l}\text { Promote research and exploitation of mines and hydrocarbon extraction } \\
\text { industries }\end{array}$ & $\begin{array}{l}\text { Publication of laws designed to promote research } \\
\text { and exploitation of mines }\end{array}$ & $\begin{array}{l}\text { Starting in } \\
2003\end{array}$ & $\begin{array}{l}\text { MINMEE } \\
\text { Economic } \\
\text { operators }\end{array}$ \\
\hline & & $\begin{array}{l}\text { Modernize and computerize the Geological and Mining Documentation } \\
\text { Center }\end{array}$ & $\begin{array}{l}\text { Creation and equiping of the Center; } \\
\text { Computerization of geological data; } \\
\text { Improvement of access to geological and mining } \\
\text { information by investors. }\end{array}$ & 2004 & MINMEE \\
\hline
\end{tabular}


Annex : Implementation matrix

STRATEGY AND MEASURES FOR REDUCTION OF POVERTY

\begin{tabular}{|c|c|c|c|c|c|}
\hline Strategy & Field/policy & Actions/measures & Performance indicators & Timetable & $\begin{array}{l}\text { Assigned } \\
\text { responsibility }\end{array}$ \\
\hline & & $\begin{array}{l}\text { Rehabilitate the Center for Analysis and Experimentation (laboratory of } \\
\text { the MINMEE) }\end{array}$ & Effective rehabilitation of the Center & $2003-2005$ & MINMEE \\
\hline & & Create a geological and mining map on a scale of $1: 200.000$ & $\begin{array}{l}\text { Creation and adoption of a detailed map; } \\
\text { Publication and dissemination of the map. }\end{array}$ & $2003-2008$ & $\begin{array}{l}\text { MINMEE } \\
\text { MINREST } \\
\text { Partners }\end{array}$ \\
\hline & & Enforce the new mining, petroleum and gas codes & $\begin{array}{l}\text { Mechanisms for monitoring compliance with the } \\
\text { new codes; } \\
\text { Effective enforcement of the new codes }\end{array}$ & Ongoing & MINMEE \\
\hline & & Seek out investments in the mining and hydrocarbon sectors & Number of investments acquired & Ongoing & $\begin{array}{l}\text { MINMEE } \\
\text { MINEPAT }\end{array}$ \\
\hline & \multicolumn{5}{|c|}{ Fight against risks and disasters with geological and industrial causes } \\
\hline & & $\begin{array}{l}\text { Strengthen risk and disaster management from geological and industrial } \\
\text { sources }\end{array}$ & $\begin{array}{l}\text { Risk and disaster management plan drawn up and } \\
\text { implemented }\end{array}$ & Ongoing & $\begin{array}{l}\text { MINMEE } \\
\text { MINREST } \\
\text { MINATD }\end{array}$ \\
\hline & & Strengthen the inspection of dangerous industrial workplaces & $\begin{array}{l}\text { Number of periodic inspections and receipts } \\
\text { generated }\end{array}$ & Ongoing & $\begin{array}{l}\text { MINMEE } \\
\text { MINEF }\end{array}$ \\
\hline & \multicolumn{5}{|c|}{ Faciliate access to energy by all members of the society } \\
\hline & & Develop and extend the program of rural electrification & Degree of access to electricity in rual areas & Yearly & $\begin{array}{l}\text { MINMEE } \\
\text { AER }\end{array}$ \\
\hline & & Develop and make accessible alternative forms of energy (solar, wind, etc.) & $\begin{array}{l}\text { Availability of technologies for use of alternative } \\
\text { forms of energy; } \\
\text { Degree of access to alternative forms of energy. }\end{array}$ & $2003-2005$ & MINMEE \\
\hline & & $\begin{array}{l}\text { Establish a rational distribution network for household gas in } \\
\text { environmentally fragile areas }\end{array}$ & $\begin{array}{l}\text { Identification of fragile environmental areas; } \\
\text { Rate of access to household gas in these areas. }\end{array}$ & $2003-2005$ & $\begin{array}{l}\text { MINMEE } \\
\text { SCDP } \\
\text { CSPH }\end{array}$ \\
\hline
\end{tabular}


Annex : Implementation matrix

STRATEGY AND MEASURES FOR REDUCTION OF POVERTY

\begin{tabular}{|c|c|c|c|c|c|}
\hline Strategy & Field/policy & Actions/measures & Performance indicators & Timetable & $\begin{array}{l}\text { Assigned } \\
\text { responsibility }\end{array}$ \\
\hline & & $\begin{array}{l}\text { Undertake a study on available options from technical, economic and } \\
\text { financial perspectives, to reduce short-term electrical power shortages that } \\
\text { may occur }\end{array}$ & $\begin{array}{l}\text { Publication of the report on the study; } \\
\text { Options chosen for reducing short-term electrical } \\
\text { power shortages that may occur. }\end{array}$ & $2003-2005$ & $\begin{array}{l}\text { MINMEE } \\
\text { ARSEL }\end{array}$ \\
\hline & \multicolumn{2}{|c|}{ Environmental management } & & & \\
\hline & & Implement the Biological Diversity Action Plan & $\begin{array}{l}\text { Effective implementation of the Biological Diversity } \\
\text { Action Plan }\end{array}$ & $\begin{array}{l}\text { Starting in } \\
2003\end{array}$ & MINEF \\
\hline \multicolumn{6}{|c|}{ Axis 5: Strengthening and enhancement of humanan resources } \\
\hline & \multicolumn{5}{|c|}{ 5.1 Implementation of the education strategy } \\
\hline & & $\begin{array}{l}\text { In collaboration with UNICEF, UNESCO and the NGOs, implement an } \\
\text { information and awareness program aimed at parents and communities, } \\
\text { describing the benefits of children's education }\end{array}$ & $\begin{array}{l}\text { Concerted awareness campaign for parents and } \\
\text { communities developed and implemented; } \\
\text { Creation of ad hoc information and awareness cells } \\
\text { for parents. }\end{array}$ & 2003 & $\begin{array}{l}\text { MINEDUC } \\
\text { MINETFOP }\end{array}$ \\
\hline & & $\begin{array}{l}\text { In timely fashion, implement a satisfactory sectoral strategy focused in } \\
\text { particular on deconcentration/decentralization of management of teachers } \\
\text { and staff involved in education }\end{array}$ & Evaluation report made available & $\begin{array}{l}\text { Starting in } \\
2003\end{array}$ & $\begin{array}{l}\text { MINEDUC } \\
\text { MINETFOP }\end{array}$ \\
\hline & & $\begin{array}{l}\text { Undertake an audit of indebtedness in private education under the auspices } \\
\text { of the CNPS; on the basis of that audit, devise appropriate solutions }\end{array}$ & Audit report made available & 2003 & $\begin{array}{l}\text { MINEDUC } \\
\text { MINETFOP }\end{array}$ \\
\hline & & $\begin{array}{l}\text { Strengthen oversight and evaluation systems at the Ministry of National } \\
\text { Education }\end{array}$ & Number of agents involved trained & 2003 & $\begin{array}{l}\text { MINEDUC } \\
\text { MINETFOP }\end{array}$ \\
\hline & & $\begin{array}{l}\text { Involve decentralized local government entities, civil society, NGOs and } \\
\text { families in the funding of preschool education }\end{array}$ & $\begin{array}{l}\text { Concerted strategy for funding preschool education } \\
\text { devised and implemented; } \\
\text { Share of other participating partners in the funding } \\
\text { of preschool education. }\end{array}$ & $\begin{array}{l}\text { Starting in } \\
2003\end{array}$ & MINEDUC \\
\hline & & Devise incentives for the creation of private schools in rural areas & $\begin{array}{l}\text { Adoption and implementation of a new law on } \\
\text { private education }\end{array}$ & 2004 & MINEDUC \\
\hline
\end{tabular}


Annex : Implementation matrix

STRATEGY AND MEASURES FOR REDUCTION OF POVERTY

\begin{tabular}{|c|c|c|c|c|c|}
\hline Strategy & Field/policy & Actions/measures & Performance indicators & Timetable & $\begin{array}{l}\text { Assigned } \\
\text { responsibility }\end{array}$ \\
\hline & & $\begin{array}{l}\text { Train the managerial staff, ensure effective oversight and supervision of the } \\
\text { operation of preschool institutions }\end{array}$ & $\begin{array}{l}\text { Identification of training needs; } \\
\text { Number of staff trained; } \\
\text { Various follow-up reports completed. }\end{array}$ & 2004 & MINEDUC \\
\hline & & $\begin{array}{l}\text { Strengthen the partnership with the private sector in the areas of construction } \\
\text { of reception centers and improvement of management of private institutions }\end{array}$ & Law adopted and implemented & $2003-2005$ & $\begin{array}{l}\text { MINEDUC } \\
\text { MINETFOP }\end{array}$ \\
\hline & & $\begin{array}{l}\text { Update the school-zone map to improve the information system of the } \\
\text { Ministry of National Education }\end{array}$ & School-zone map updated and published & Ongoing & MINEDUC \\
\hline & & $\begin{array}{l}\text { Make certain that qualified teachers are actually present in all the schools, } \\
\text { including those in the least advantaged regions }\end{array}$ & Actual presence of the teachers & Ongoing & MINEDUC \\
\hline & & $\begin{array}{l}\text { Expand government action on behalf of private educational institutions } \\
\text { (subsidies, assignment of teachers, improvement of infrastructures) }\end{array}$ & $\begin{array}{l}\text { Amount of subsidies granted to private eduction; } \\
\text { Number of teachers from public education } \\
\text { assigned to private educational institutions. }\end{array}$ & 2004 & MINEDUC \\
\hline & & $\begin{array}{l}\text { Institute regular production of statistical charts at all levels of the educational } \\
\text { system }\end{array}$ & $\begin{array}{l}\text { Periodic production of a statistical yearbook by } \\
\text { level of instruction }\end{array}$ & 2004 & MINEDUC \\
\hline & & $\begin{array}{l}\text { Promote access to information and communications technologies in the } \\
\text { educational system }\end{array}$ & $\begin{array}{l}\text { Number of pilot lycées equipped with computer } \\
\text { systems; } \\
\text { Number of students instructed in use of computers }\end{array}$ & 2004 & MINEDUC \\
\hline & & Provide technical, technological and career training & $\begin{array}{l}\text { Number of training sessions offered; } \\
\text { Number of students trained. }\end{array}$ & $2003-2004$ & MINESUP \\
\hline
\end{tabular}


Annex : Implementation matrix

STRATEGY AND MEASURES FOR REDUCTION OF POVERTY

\begin{tabular}{|c|c|c|c|c|c|}
\hline Strategy & Field/policy & Actions/measures & Performance indicators & Timetable & $\begin{array}{l}\text { Assigned } \\
\text { responsibility }\end{array}$ \\
\hline & & $\begin{array}{l}\text { Undertake a study of implementing distance training, both as an alternative } \\
\text { method and on an ongoing basis }\end{array}$ & $\begin{array}{l}\text { Report on the study with approaches indicated; } \\
\text { Identification of distance training needs, both as } \\
\text { an alternative method and on an ongoing basis, } \\
\text { both functional and accessible. }\end{array}$ & $2003-2004$ & MINESUP \\
\hline & & $\begin{array}{l}\text { Undertake an architectural and cost-analysis study for construction and } \\
\text { rehabilitation of academic infrastructures }\end{array}$ & $\begin{array}{l}\text { Study report made available; } \\
\text { Construction and rehabilitation costs determined. }\end{array}$ & 2004 & MINESUP \\
\hline & & Equip institutions of higher learning with computer systems & $\begin{array}{l}\text { Number of institutions equiped; } \\
\text { Degree of student access to computer systems }\end{array}$ & $2003-2004$ & MINESUP \\
\hline & \multicolumn{5}{|c|}{5.2 Implementation of the health strategy } \\
\hline & \multicolumn{2}{|c|}{ Fight against malaria } & & & \\
\hline & & $\begin{array}{l}\text { Ensure training and updating of skills of healthcare providers responsible for } \\
\text { patient case management in health districts }\end{array}$ & $\begin{array}{l}\text { At least } 90 \text { percent of healthcare providers with } \\
\text { updated skills as regards case management of } \\
\text { patients }\end{array}$ & 2003 & MINSANTE \\
\hline & & Provide home patientcare kits to households in the 44 health districts & $\begin{array}{l}\text { At least } 50 \text { percent of households in possession of } \\
\text { home patientcare kits }\end{array}$ & 2003 & MINSANTE \\
\hline & & $\begin{array}{l}\text { Gradually increase to } 80 \text { percent the rate of healthcare training programs that } \\
\text { include management of cases of malaria }\end{array}$ & $\begin{array}{l}\text { At least } 80 \text { percent of healthcare training } \\
\text { programs equipped with documentation for } \\
\text { managing cases of malaria }\end{array}$ & $\begin{array}{l}\text { Starting in } \\
2003\end{array}$ & MINSANTE \\
\hline & & $\begin{array}{l}\text { Adopt and publish national directives regarding the integrated fight against } \\
\text { desease vectors }\end{array}$ & $\begin{array}{l}\text { Adoption and publication of the national } \\
\text { directives }\end{array}$ & 2003 & MINSANTE \\
\hline & & $\begin{array}{l}\text { Provide training to members of information agencies or community agents } \\
\text { regarding soaking of mosquito nets with insecticide }\end{array}$ & Number of community agents trained & 2003 & MINSANTE \\
\hline & & Draw up and adopt a national plan for information on malaria & Information plan adopted and implemented & 2003 & MINSANTE \\
\hline & & $\begin{array}{l}\text { Provide at least } 50 \text { percent of pregnant women with mosquito netting soaked } \\
\text { with insecticide }\end{array}$ & $\begin{array}{l}\text { Effective distribution of the } 150,000 \text { mosquito } \\
\text { nets acquired in } 2002 \text { and start of distribution of } \\
\text { the } 660,000 \text { nets acquired in } 2003 \text {. }\end{array}$ & $2003-2004$ & MINSANTE \\
\hline
\end{tabular}


Annex : Implementation matrix

STRATEGY AND MEASURES FOR REDUCTION OF POVERTY

\begin{tabular}{|c|c|c|c|c|c|}
\hline Strategy & Field/policy & Actions/measures & Performance indicators & Timetable & $\begin{array}{l}\text { Assigned } \\
\text { responsibility }\end{array}$ \\
\hline & \multicolumn{2}{|c|}{ Endemic and catastrophic emergencies } & & & \\
\hline & & $\begin{array}{l}\text { Create and make operational the national center and at least two secondary } \\
\text { centers for the treatment of emergencies }\end{array}$ & Operational national center and secondary centers & 2003 & MINSANTE \\
\hline & \multicolumn{2}{|r|}{ 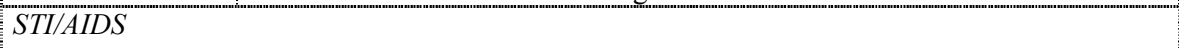 } & & & \\
\hline & & $\begin{array}{l}\text { Promote the use of contraceptives among truck drivers, dock workers, } \\
\text { soldiers and prostitutes }\end{array}$ & Purchase and distribution of condoms & 2003 & MINSANTE \\
\hline & & Create an HIV counseling and testing center in each province & Number of operational provincial centers created & 2003 & MINSANTE \\
\hline & & Provide private, not for profit hospitals with consumable reagents and ARVs & $\begin{array}{l}\text { Number of private, not for profit hospitals } \\
\text { provided with consumable and reagents and } \\
\text { ARVs }\end{array}$ & 2003 & MINSANTE \\
\hline & & $\begin{array}{l}\text { Implement a broadly-based awareness campaign on the prevention and } \\
\text { transmission from mother to child (PTME) of STI/AIDS }\end{array}$ & $\begin{array}{l}\text { Number of copies of technical prevention guides } \\
\text { produced in French and English and distributed to } \\
\text { all maternity wards and CSIs }\end{array}$ & 2003 & MINSANTE \\
\hline & & Provide the health centers with HIV/AIDS diagnostic equipment & $\begin{array}{l}\text { Number of healthcare service centers, units, blood } \\
\text { transfusion centers, blood banks and depositories } \\
\text { in possession of tests for HIV, hepatitis B and C } \\
\text { and syphilis. }\end{array}$ & 2003 & MINSANTE \\
\hline & \multicolumn{2}{|c|}{ Fight against tuberculosis } & & & \\
\hline & & $\begin{array}{l}\text { Undertake architectural studies for the construction of an operating diagnostic } \\
\text { and treatment center for } 50,000 \text { to } 100,000 \text { inhabitants, in each province }\end{array}$ & Studies available for each province & 2003 & MINSANTE \\
\hline & & $\begin{array}{l}\text { Disseminate widely the use of the Technical Guide for the care of } \\
\text { tuberculosis patients in the diagnostic and treatment centers. }\end{array}$ & $\begin{array}{l}\text { Number of diagnostic and treatment centers in } \\
\text { possession of a Technical Guide for the treatment } \\
\text { of tuberculosis cases; } \\
\text { Number of cases of tuberculosis identified and } \\
\text { treated. }\end{array}$ & 2003 & MINSANTE \\
\hline
\end{tabular}


Annex : Implementation matrix

STRATEGY AND MEASURES FOR REDUCTION OF POVERTY

\begin{tabular}{|c|c|c|c|c|c|}
\hline Strategy & Field/policy & Actions/measures & Performance indicators & Timetable & $\begin{array}{l}\text { Assigned } \\
\text { responsibility }\end{array}$ \\
\hline & & Equip the CDTs with functioning microscopes. & $\begin{array}{l}\text { Number of CDTs possessing a functioning } \\
\text { microscope }\end{array}$ & 2003 & MINSANTE \\
\hline & & $\begin{array}{l}\text { Train or update skills of current doctors, nurses and laboratory assistants in } \\
\text { the management of tuberculosis patients in each existing CDT }\end{array}$ & $\begin{array}{l}\text { Number of doctors, nurses and laboratory } \\
\text { assistants trained in each existing CDT }\end{array}$ & 2003 & MINSANTE \\
\hline & & Draw up and adopt a national tuberculosis information plan & Available, operational plan & 2003 & MINSANTE \\
\hline & \multicolumn{2}{|c|}{ Expanded vaccination program $(P E V)$} & & & \\
\hline & & Broaden the vaccination coverage at DTC3 & Rate of vaccination coverage & 2003 & MINSANTE \\
\hline & & Equip the healthcare facilities with cold chains & $\begin{array}{l}\text { Number of facilities possessing a functioning cold } \\
\text { chain }\end{array}$ & 2003 & MINSANTE \\
\hline & & Strengthen the logistic resources of the vaccination teams & $\begin{array}{l}\text { Number of motorcycles acquired; } \\
\text { Number of vaccination teams possessing a } \\
\text { motorcycle. }\end{array}$ & 2003 & MINSANTE \\
\hline & \multicolumn{2}{|c|}{ Drugs, reagents and basic medical supplies } & & & \\
\hline & & $\begin{array}{l}\text { Make basic drugs, preferably in generic form, available in all healthcare } \\
\text { facilities; make available reagents and basic medical supplies }\end{array}$ & $\begin{array}{l}\text { Number of healthcare facilities possessing basic } \\
\text { drugs, reagents and basic medical supplies }\end{array}$ & 2003 & MINSANTE \\
\hline & & Develop and make available in the healthcare facilities a guide to therapy & Guides available in healthcare facilities & 2003 & MINSANTE \\
\hline & & $\begin{array}{l}\text { Draw up and make available in all healthcare facilities a national basic drugs } \\
\text { order form }\end{array}$ & $\begin{array}{l}\text { Number of healthcare facilities in possession of } \\
\text { the national basic drugs order form }\end{array}$ & 2003 & MINSANTE \\
\hline & \multicolumn{2}{|c|}{ Healthcare funding } & & & \\
\hline & & Draw up a Mutual Insurance Code & $\begin{array}{l}\text { Creation, publication and dissemination of the } \\
\text { Mutual Insurance Code }\end{array}$ & 2003 & MINSANTE \\
\hline
\end{tabular}


Annex : Implementation matrix

STRATEGY AND MEASURES FOR REDUCTION OF POVERTY

\begin{tabular}{|c|c|c|c|c|c|}
\hline Strategy & Field/policy & Actions/measures & Performance indicators & Timetable & $\begin{array}{l}\text { Assigned } \\
\text { responsibility }\end{array}$ \\
\hline & \multicolumn{5}{|c|}{ Health of mothers, adolescents and the elderly } \\
\hline & & Promote vaccination of pregnant women visiting clinincs against tetanus & $\begin{array}{l}\text { Number of pregnant women seen in clinics and } \\
\text { vaccinated against tetanus }\end{array}$ & 2003 & MINSANTE \\
\hline & & Improve the rate of births assisted by qualified personnel & $\begin{array}{l}\text { Number of births in the presence of qualified } \\
\text { personnel }\end{array}$ & 2003 & MINSANTE \\
\hline & & Systematic diagnosis of breast and cervical cancers & $\begin{array}{l}\text { Number of breast and cervical cancers diagnosed } \\
\text { and treated }\end{array}$ & 2003 & MINSANTE \\
\hline & \multicolumn{5}{|c|}{ Promotion of Integrated Management of Children's Illnesses (PCIME) } \\
\hline & & Implement PCIME in the health districts & $\begin{array}{l}\text { Number of health districts actively engaged in } \\
\text { PCIME }\end{array}$ & 2003 & MINSANTE \\
\hline & & $\begin{array}{l}\text { Train physician heads of health districts and heads of participating healthcare } \\
\text { centers in PCIME }\end{array}$ & $\begin{array}{l}\text { Number of physician heads of health districts and } \\
\text { heads of participating healthcare centers trained in } \\
\text { PCIME }\end{array}$ & 2003 & MINSANTE \\
\hline & & Develop a national information, education and communication plan & Available, working plan & 2003 & MINSANTE \\
\hline & \multicolumn{2}{|c|}{ Diet and nutrition } & & & \\
\hline & & Draw up a national nutrition policy document & $\begin{array}{l}\text { Development, publication and dissemination of } \\
\text { the national nutrition policy document }\end{array}$ & 2003 & MINSANTE \\
\hline & & $\begin{array}{l}\text { Provide children aged six months to } 5 \text { years with vitamin A capsules two } \\
\text { times a year }\end{array}$ & $\begin{array}{l}\text { Number of children aged six months to } 5 \text { years } \\
\text { provided with vitamin A capsules two times a } \\
\text { year }\end{array}$ & 2003 & MINSANTE \\
\hline & & $\begin{array}{l}\text { Ensure that first-tier health care service providers oversee the growth of } \\
\text { young children }\end{array}$ & $\begin{array}{l}\text { At least } 25 \text { percent of first-tier health care service } \\
\text { providers overseeing the growth of young } \\
\text { children }\end{array}$ & 2003 & MINSANTE \\
\hline & \multicolumn{2}{|c|}{ Improve the quality of healthcare services provided } & & & \\
\hline & & Rehabilitate training institutions & $\begin{array}{l}\text { At least } 25 \text { percent of training institutions } \\
\text { rehabilitated and operating }\end{array}$ & 2003 & MINSANTE \\
\hline & & $\begin{array}{l}\text { Evaluate the qualitative and quantitative assets of } 40 \text { percent of the health } \\
\text { districts for compliance with the priorities set forth in the Health Map }\end{array}$ & $\begin{array}{l}\text { Completed report of a study of } 40 \text { percent of the } \\
\text { health districts available }\end{array}$ & 2003 & MINSANTE \\
\hline & & $\begin{array}{l}\text { Construct new integrated healthcare centers (CSIs) in the healthcare regions } \\
\text { not currently covered }\end{array}$ & Number of new CSIs built & 2003 & MINSANTE \\
\hline
\end{tabular}


Annex : Implementation matrix

STRATEGY AND MEASURES FOR REDUCTION OF POVERTY

\begin{tabular}{|c|c|c|c|c|c|}
\hline Strategy & Field/policy & Actions/measures & Performance indicators & Timetable & $\begin{array}{l}\text { Assigned } \\
\text { responsibility }\end{array}$ \\
\hline & \multicolumn{2}{|c|}{ Management process } & & \multirow[b]{2}{*}{2003} & \multirow[b]{2}{*}{ MINSANTE } \\
\hline & & $\begin{array}{l}\text { Train the directors of healthcare districts, the physician heads of district } \\
\text { hospitals, and the assistant directors of central health services and outpatient } \\
\text { services, in the techniques for drawing up and managing integrated budgets } \\
\text { targeted on objectives. }\end{array}$ & $\begin{array}{l}\text { At least } 25 \text { percent of directors of healthcare } \\
\text { districts, physician heads of district hospitals, and } \\
\text { assistant directors of central health services and } \\
\text { outpatient services trained }\end{array}$ & & \\
\hline & & Recrute healthcare staff under contracts specific to assigned locations & $\begin{array}{l}\text { At least } 1,200 \text { healthcare staff under contracts } \\
\text { specific to assigned locations }\end{array}$ & 2003 & MINSANTE \\
\hline & & Build residences for doctors practicing in disadvantaged areas & $\begin{array}{l}\text { At least } 100 \text { residences for doctors practicing in } \\
\text { disadvantaged areas }\end{array}$ & 2003 & MINSANTE \\
\hline & \multicolumn{2}{|c|}{5.3 Other social development policies and strategies } & & & \\
\hline & & Finalize the social development strategy & $\begin{array}{l}\text { Finalization, publication and dissemination of the } \\
\text { strategy }\end{array}$ & 2003 & $\begin{array}{l}\text { MINEPAT } \\
\text { Sectoral } \\
\text { ministries }\end{array}$ \\
\hline & & Finalize the strategy for promotion of women & $\begin{array}{l}\text { Finalization, publication and dissemination of the } \\
\text { strategy }\end{array}$ & 2003 & MINCOF \\
\hline & & Adopt a law for protection of the rights and dignity of women & $\begin{array}{l}\text { Finalization, publication and dissemination of the } \\
\text { law to protect the rights and dignity of women }\end{array}$ & $2003-2005$ & MINCOF \\
\hline & & Promote the informal education of women and girls & $\begin{array}{l}\text { Progress made in the reduction of the school drop- } \\
\text { out rate and in illiteracy among girls and women }\end{array}$ & $2003-2005$ & MINCOF \\
\hline & & $\begin{array}{l}\text { Strengthen resources of field agents in the area of promotion of women } \\
\text { within specialized institutions (women's support centers and applied } \\
\text { technology centers) }\end{array}$ & $\begin{array}{l}\text { Enhancement program for strengthening resources } \\
\text { drawn up and implemented; } \\
\text { Organization of seminars and training sessions; } \\
\text { Number of field agents trained. }\end{array}$ & $2003-2005$ & MINCOF \\
\hline & & Finalize the sectoral strategy of the social affairs sector & $\begin{array}{l}\text { Adoption, publication and dissemination of the } \\
\text { strategy }\end{array}$ & 2003 & MINAS \\
\hline & & Finalize the Code of Persons and Families & $\begin{array}{l}\text { Publication and dissemination of the Code of } \\
\text { Persons and Families }\end{array}$ & 2003 & MINAS \\
\hline & & Adopt a law on the protection of children & Publication and dissemination of the law & 2004 & minas \\
\hline & & Adopt a law promoting the integration of marginalized populations & $\begin{array}{l}\text { Publication of the law promoting the integration } \\
\text { of marginalized populations }\end{array}$ & 2004 & minas \\
\hline & & $\begin{array}{l}\text { Renovate institutions for rehabilitation of handicapped children on public } \\
\text { assistance }\end{array}$ & Number of institutions renovated & 2004 & minas \\
\hline & & $\begin{array}{l}\text { Develop social services for the social and economic integration of } \\
\text { handicapped persons }\end{array}$ & $\begin{array}{l}\text { Number of plans developed each year; } \\
\text { Number of handicapped persons trained and } \\
\text { integrated. }\end{array}$ & Ongoing & minas \\
\hline
\end{tabular}


Annex : Implementation matrix

STRATEGY AND MEASURES FOR REDUCTION OF POVERTY

\begin{tabular}{|c|c|c|c|c|c|}
\hline Strategy & Field/policy & Actions/measures & Performance indicators & Timetable & $\begin{array}{l}\text { Assigned } \\
\text { responsibility }\end{array}$ \\
\hline & & $\begin{array}{l}\text { Promote social services in open custody facilities for minor children in need } \\
\text { of public protection }\end{array}$ & $\begin{array}{l}\text { Service plans drawn up each year; } \\
\text { Number of minor children served, trained and } \\
\text { integrated. }\end{array}$ & Ongoing & minas \\
\hline & & $\begin{array}{l}\text { Promote the expansion of local self-help initiatives for support and training of } \\
\text { families }\end{array}$ & $\begin{array}{l}\text { Strategy for promotion of local initiatives drawn } \\
\text { up and implemented; } \\
\text { Number of initiatives supported each year. }\end{array}$ & Ongoing & minas \\
\hline & \multicolumn{5}{|c|}{5.4 Reduction of urban poverty } \\
\hline & \multirow[t]{9}{*}{ n } & Finalize the sectoral strategy for urban development & $\begin{array}{l}\text { Finalization, publication and dissemination of the } \\
\text { strategy }\end{array}$ & 2003 & MINVILLE \\
\hline & & Undertake a study on development of a national housing policy & $\begin{array}{l}\text { Report on the study made available and used in } \\
\text { formulating national housing policy }\end{array}$ & 2004 & MINUH \\
\hline & & Rehabilitate waste water treatment facilities & Number of facilities rehabilitated & $\begin{array}{l}\text { Starting in } \\
2003\end{array}$ & $\begin{array}{l}\text { MINVILLE } \\
\text { MINUH } \\
\text { CUY } \\
\text { CUD }\end{array}$ \\
\hline & & $\begin{array}{l}\text { Undertake restoration of primary drainage networks, rehabilitate waste water } \\
\text { treatment facilities }\end{array}$ & Surface dimensions of network drained & Ongoing & $\begin{array}{l}\text { MINVILLE } \\
\text { MINUH } \\
\text { CUY } \\
\text { CUD }\end{array}$ \\
\hline & & Rehabilitate essential infrastructures (road system, public lighting) & Measured amount rehabilitated & Ongoing & $\begin{array}{l}\text { MINVILLE } \\
\text { MINUH/CUY } \\
\text { CUD }\end{array}$ \\
\hline & & $\begin{array}{l}\text { Undertake specific studies in view of creating a coherent and workable action } \\
\text { plan for fighting urban poverty }\end{array}$ & $\begin{array}{l}\text { Availability of reports on specific studies; } \\
\text { Coherent and workable action plan for fighting } \\
\text { urban poverty drawn up. }\end{array}$ & 2004 & MINVILLE \\
\hline & & $\begin{array}{l}\text { Implement urgent plans for restoring health to the urban communities of } \\
\text { Yaoundé and Douala }\end{array}$ & Action plans drawn up and implemented & 2004 & $\begin{array}{l}\text { MINVILLE } \\
\text { CUY/CUD }\end{array}$ \\
\hline & & Organize urban extension zones and secondary urban centers & $\begin{array}{l}\text { Surfaces organized and development plans drawn } \\
\text { up }\end{array}$ & 2005 & $\begin{array}{l}\text { MINVILLE } \\
\text { MINUH }\end{array}$ \\
\hline & & $\begin{array}{l}\text { Put on a contractual basis the partnership between the state and decentralized } \\
\text { territorial governmental units in order to improve the management of cities }\end{array}$ & $\begin{array}{l}\text { Number of city contracts entered into and } \\
\text { implemented }\end{array}$ & 2005 & $\begin{array}{l}\text { MINVILLE } \\
\text { MINUH }\end{array}$ \\
\hline
\end{tabular}


Annex : Implementation matrix

STRATEGY AND MEASURES FOR REDUCTION OF POVERTY

\begin{tabular}{|c|c|c|c|c|c|}
\hline Strategy & Field/policy & Actions/measures & Performance indicators & Timetable & $\begin{array}{l}\text { Assigned } \\
\text { responsibility }\end{array}$ \\
\hline & & $\begin{array}{l}\text { Remove barriers isolating poorly served neighborhoods and reorganize } \\
\text { cahotic residential zones }\end{array}$ & $\begin{array}{l}\text { - Urbanization plan drawn up and implemented; } \\
\text { - Number of streets replacing barriers } \\
\text { rehabilitated or created; } \\
\text { - Number of chatoic neighborhoods reorganized } \\
\text { in consultation with resident populations }\end{array}$ & 2005 & $\begin{array}{l}\text { MINVILLE } \\
\text { CUY/CUD }\end{array}$ \\
\hline & & Create centers to serve street children, aimed at returning them to society & $\begin{array}{l}\text { Number of reception centers created; } \\
\text { Number of street children helped and returned to } \\
\text { society }\end{array}$ & $2003-2005$ & $\begin{array}{l}\text { MINVILLE } \\
\text { CUY/CUD }\end{array}$ \\
\hline & & Take measures to prevent urban delinquency & Strategies drawn up and implemented & $2003-2004$ & $\begin{array}{l}\text { MINVILLE } \\
\text { CUY/CUD }\end{array}$ \\
\hline & & Draw up a transportation plan for Yaoundé and Douala & $\begin{array}{l}\text { Creation, adoption and publication of the } \\
\text { transportation plan for Yaoundé and Douala }\end{array}$ & $2003-2004$ & $\begin{array}{l}\text { MINVILLE } \\
\text { CUY/CUD }\end{array}$ \\
\hline & & Plan the controlled discharge of household waste & Number of discharges planned & 2005 & $\begin{array}{l}\text { MINVILLE } \\
\text { CUY/CUD }\end{array}$ \\
\hline & \multicolumn{5}{|c|}{5.5 Job creation and effective reintegration into the economy of disadvantaged groups } \\
\hline & & Finalize and adopt the Declaration of National Employment Policy & $\begin{array}{l}\text { Adoption, publication and dissemination of the } \\
\text { Declaration }\end{array}$ & 2003 & METPS \\
\hline & & Undertake a study on employment in the informal sector & Publication of the report on the study & 2003 & ONEFOP /INS \\
\hline & & $\begin{array}{l}\text { Promote the implementation of information systems on the employment } \\
\text { market }\end{array}$ & $\begin{array}{l}\text { Implementation of the information system; } \\
\text { Ongoing data made availabile. }\end{array}$ & Ongoing & ONEFOP /INS \\
\hline & & Promote the employment of youth & Number of youth brought into the workforce & Ongoing & FNE \\
\hline \multicolumn{6}{|c|}{ Axis 6: Improvement of the institutional framework and governance } \\
\hline & \multicolumn{2}{|c|}{$\begin{array}{l}\text { Increased transparency in the management of public affairs and improved accountability of } \\
\text { managers }\end{array}$} & & & \\
\hline & & $\begin{array}{l}\text { Publish the results of studies, inquiries and audits undertaken in view of } \\
\text { improving the provision of services in the key sectors (education, health, } \\
\text { basic infrastructures, justice) }\end{array}$ & $\begin{array}{l}\text { Results of inquiries and audits published and } \\
\text { made available to the public }\end{array}$ & 2003 & $\begin{array}{l}\text { MINEPAT } \\
\text { MINEDUC } \\
\text { MINSANTE }\end{array}$ \\
\hline & & $\begin{array}{l}\text { Undertake periodic budget follow-ups in the social sectors, using audits made } \\
\text { by independent consultants }\end{array}$ & $\begin{array}{l}\text { Results of inquiries and audits published and } \\
\text { made available to the public }\end{array}$ & Ongoing & $\begin{array}{l}\text { MINEPAT } \\
\text { MINEDUC } \\
\text { MINSANTE }\end{array}$ \\
\hline & & $\begin{array}{l}\text { Make an annual audit of the public procurement system by independent } \\
\text { consultants }\end{array}$ & $\begin{array}{l}\text { Audit reports published and made available to the } \\
\text { public }\end{array}$ & Ongoing & ARMP \\
\hline
\end{tabular}


Annex : Implementation matrix

STRATEGY AND MEASURES FOR REDUCTION OF POVERTY

\begin{tabular}{|c|c|c|c|c|c|}
\hline Strategy & Field/policy & Actions/measures & Performance indicators & Timetable & $\begin{array}{l}\text { Assigned } \\
\text { responsibility }\end{array}$ \\
\hline & \multicolumn{2}{|c|}{ Continuation of the in-depth reform of the justice system } & & & \\
\hline & & Complete the technical audit of the justice system & Study report made available & 2003 & $\begin{array}{l}\text { MINJUSTICE } \\
\text { MINEPAT }\end{array}$ \\
\hline & & $\begin{array}{l}\text { Draw up and implement the strategy for reform of the justice system based on } \\
\text { the recommendations of the technical audit of the justice system }\end{array}$ & Strategy adopted and implemented & $2003-2004$ & $\begin{array}{l}\text { MINJUSTICE } \\
\text { MINEPAT }\end{array}$ \\
\hline & & $\begin{array}{l}\text { Establish the jurisdictional units called for in the Constitution of January } \\
\text { 1996, specifically the Chambre des Comptes and the Conseil Constitutionnel }\end{array}$ & $\begin{array}{l}\text { Law organizing the Chambre des Comptes and } \\
\text { the Conseil Constitutionnel; } \\
\text { Chambre des Comptes and Conseil } \\
\text { Constitutionnel both operational. }\end{array}$ & $2003-2004$ & $\begin{array}{l}\text { MINJUSTICE } \\
\text { MINFI } \\
\text { MINEPAT }\end{array}$ \\
\hline & \multicolumn{2}{|c|}{ Step up the fight against corruption } & & & \\
\hline & & $\begin{array}{l}\text { Form a coalition for fighting corruption involving the public authorities, the } \\
\text { private sector, civil society and all the actors with interests in the fight }\end{array}$ & $\begin{array}{l}\text { Existence of a working coalition; } \\
\text { Action program drawn up in collaboration with } \\
\text { civil society. }\end{array}$ & 2003 & PNG \\
\hline & & $\begin{array}{l}\text { Draw up and adopt a strategy for making the general public aware of matters } \\
\text { related to governance and the fight against corruption }\end{array}$ & $\begin{array}{l}\text { Strategu adopted; } \\
\text { Action program drawn up. }\end{array}$ & 2003 & PNG \\
\hline & \multicolumn{2}{|c|}{ Improve the circulation and accessibility of information on the management of public affairs } & & & \\
\hline & & $\begin{array}{l}\text { Undertake a study on citizen access to information on the management of } \\
\text { public affairs }\end{array}$ & Finalized and disseminated study & 2003 & MINCOM \\
\hline & \multicolumn{2}{|c|}{ Strengthen local development by adopting some twenty community projects } & & & \\
\hline & & Select and make operational the twenty or so projects chosen & $\begin{array}{l}\text { Selection criteria defined; } \\
20 \text { operational projects. }\end{array}$ & 2003 & MINATD \\
\hline & \multicolumn{2}{|c|}{ Improve the production and dissemination of information } & & & \\
\hline & & Make the Institut National de la Statistique operational & Working units in place & 2003 & MINEPAT \\
\hline & & Evaluate the cost of implementing the Minimum Statistics Program (PSM) & Specifications for the PSM & 2003 & MINEPAT \\
\hline & \multicolumn{2}{|c|}{ Develop the partnership and the participatory process for executing the Strategy } & & & \\
\hline & & $\begin{array}{l}\text { Set up an information system for the various participants in the actions to } \\
\text { reduce poverty }\end{array}$ & Information system in place & 2003 & MINEPAT \\
\hline & & $\begin{array}{l}\text { Strengthen the resources of civil society, the private sector and government } \\
\text { personnel involved in the fight against poverty }\end{array}$ & Number of persons trained & 2003 & MINEPAT \\
\hline
\end{tabular}




\section{ANNEX 2 CORE POVERTY MONITORING INDICATORS AND STATISTICAL} PROGRAM

\section{Annex 2.1 Core indicators}

\begin{tabular}{|c|c|c|c|c|c|}
\hline Areas & Objectives & Indicators & Source of data & $\begin{array}{l}\text { Responsible } \\
\text { agencies }\end{array}$ & Periodicity \\
\hline \multirow{3}{*}{ 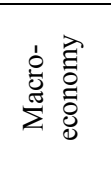 } & \multirow{2}{*}{$\begin{array}{l}\text { Measuring the creation of } \\
\text { wealth }\end{array}$} & $\begin{array}{ll}\text { 1. } & \text { GDP }\end{array}$ & National accounts $(\mathrm{CN})$ & INS & Annual \\
\hline & & 2. Real growth rate & National accounts $(\mathrm{CN})$ & INS & Annual \\
\hline & Measuring trends in prices & 3. Inflation rate & PRICE & INS & $\begin{array}{l}\text { Monthly,/ } \\
\text { Annual }\end{array}$ \\
\hline \multirow{3}{*}{ 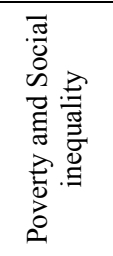 } & Measuring poverty & 4. $\quad$ Poverty incidence $(\%)$ & ECAM & INS & Five years \\
\hline & $\begin{array}{l}\text { Monitoring the gap between } \\
\text { income of the poor and } \\
\text { poverty line }\end{array}$ & 5. $\quad$ Poverty depth & ECAM & INS & Five years \\
\hline & $\begin{array}{l}\text { Monitoring inequality of the } \\
\text { poor }\end{array}$ & 6. Poverty severity & ECAM & INS & Five years \\
\hline \multirow{7}{*}{ 总 } & \multirow{3}{*}{$\begin{array}{l}\text { Measuring school } \\
\text { coverage/enrollment and } \\
\text { promoting gender equality }\end{array}$} & 7. Gross enrollment & ECAM / School map & $\begin{array}{l}\text { INS/MINEDU } \\
\mathrm{C}\end{array}$ & Annual \\
\hline & & 8. $\quad$ Primary enrollment & ECAM / School map & \begin{tabular}{|l|} 
INS/MINEDU \\
$\mathrm{C}$ \\
\end{tabular} & Annual \\
\hline & & 9. $\quad$ Primary completion rate & ECAM / School map & $\begin{array}{l}\text { INS/MINEDU } \\
\mathrm{C} \\
\end{array}$ & Annual \\
\hline & & $\begin{array}{l}\text { 10. Gender parity index in } \\
\text { primary \& secondary }\end{array}$ & $\begin{array}{l}\text { EACM/Administrative } \\
\text { statements }\end{array}$ & MINEDUC & Annual \\
\hline & $\begin{array}{l}\text { Measuring Government } \\
\text { budgeting }\end{array}$ & $\begin{array}{l}\text { 11. Share of education in } \\
\text { national budget }(\%)\end{array}$ & National budget & MINFI & Annual \\
\hline & $\begin{array}{l}\text { Measuring quality of } \\
\text { educational service }\end{array}$ & 12. Teacher pupils ratio & School map & MINEDUC & Annual \\
\hline & & 13. Pupils classroom ratio & School map & MINEDUC & Annual \\
\hline 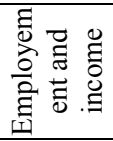 & $\begin{array}{l}\text { Measuring presence on labor } \\
\text { market }\end{array}$ & $\begin{array}{l}\text { 14. Unemployment rate (men } \\
\text { and women) }\end{array}$ & $\begin{array}{l}\text { ECAM / Employment } \\
\text { survey }\end{array}$ & INS/METPS & Annual \\
\hline
\end{tabular}




\begin{tabular}{|c|c|c|c|c|c|}
\hline Areas & Objectives & Indicators & Source of data & \begin{tabular}{|l|}
$\begin{array}{l}\text { Responsible } \\
\text { agencies }\end{array}$ \\
\end{tabular} & Periodicity \\
\hline \multirow{8}{*}{ 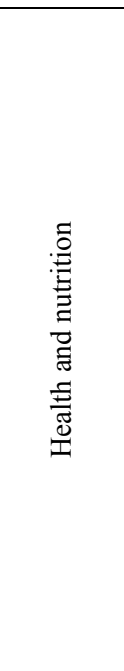 } & $\begin{array}{l}\text { Measuring children } \\
\text { immunization coverage }\end{array}$ & $\begin{array}{l}\text { 15. Infants (less than 1) } \\
\text { immunization rates }\end{array}$ & ECAM/RGPH/DHS & INS/BUCREP & Five years $^{22}$ \\
\hline & \multirow[t]{2}{*}{ Improving maternal health } & $\begin{array}{l}\text { 16. Ratio of assisted birth } \\
\text { delivery }\end{array}$ & ECAM/DHS & INS/BUCREP & Five years \\
\hline & & 17. Maternal mortality rate & ECAM/DHS & INS/BUCREP & Five years \\
\hline & $\begin{array}{l}\text { Measuring progress in infant } \\
\text { mortality reduction }\end{array}$ & 18. Infant mortality rate $(\% 0)$ & $\begin{array}{l}\text { DHS/ECAM/Administrat } \\
\text { ive statements }\end{array}$ & $\begin{array}{l}\text { INS/BUCREP/ } \\
\text { MINSANTE }\end{array}$ & Five years \\
\hline & $\begin{array}{l}\text { Measuring progress in } \\
\text { children mortality reduction }\end{array}$ & 19. Children mortality rate & DHS & INS/ BUCREP & Five years \\
\hline & $\begin{array}{l}\text { Measuring government's } \\
\text { effort in the financing of } \\
\text { health care }\end{array}$ & $\begin{array}{l}\text { 20. Share of health care in } \\
\text { national budget }\end{array}$ & National budget & MINFI/DB & Annual \\
\hline & \multirow[t]{2}{*}{$\begin{array}{l}\text { Monitoring the HIV/AIDS } \\
\text { pandemic and malaria }\end{array}$} & $\begin{array}{l}\text { 21. HIV/AIDS prevalence rate } \\
\text { among sexually active } \\
\text { population (15-49 years old) }\end{array}$ & DHS & $\begin{array}{l}\text { INS/BUCREP/ } \\
\text { MINSANTE }\end{array}$ & Five years \\
\hline & & $\begin{array}{l}\text { 22. Malaria prevalence in targt } \\
\text { groups (pregnant women and } \\
\text { less than } 5 \text { children) }\end{array}$ & DHS & INSANTE & Five years \\
\hline & Eliminate hunger & 23. Wasting rate among children & ECAM/DHS & $\begin{array}{l}\text { INS/BUCREP/ } \\
\text { MINSANTE }\end{array}$ & Five years \\
\hline & & $\begin{array}{l}\text { 24. Malnutrition rate among } \\
\text { children }\end{array}$ & ECAM/DHS & $\begin{array}{l}\text { INS/BUCREP/ } \\
\text { MINSANTE }\end{array}$ & Five years \\
\hline & & 25. Underweight children ratio & ECAM/DHS & $\begin{array}{l}\text { INS/BUCREP/ } \\
\text { MINSANTE }\end{array}$ & Five years \\
\hline \multirow[b]{2}{*}{ 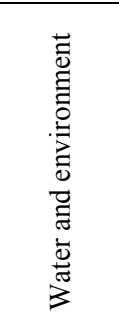 } & $\begin{array}{l}\text { Measuring access to drinking } \\
\text { water }\end{array}$ & $\begin{array}{l}\text { 26. Percentage of households } \\
\text { with access to drinking water }\end{array}$ & ECAM & INS & Five years \\
\hline & Improving standard of living & $\begin{array}{l}\text { 27. Share of households with } \\
\text { access to power }\end{array}$ & ECAM & INS & Five years \\
\hline \multirow{2}{*}{ 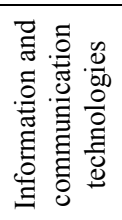 } & \multirow[t]{2}{*}{ Improving access to ICT } & $\begin{array}{l}\text { 28. Number of telephone lines } \\
\text { per } 1000 \text { households }\end{array}$ & ECAM & INS & Five years \\
\hline & & $\begin{array}{l}\text { 29. Number of computer per } \\
1.000 \text { households }\end{array}$ & ECAM & INS & Five years \\
\hline
\end{tabular}

${ }^{22}$ ECAM et DHS planed every 5 years 
Annex

Annex 2.2 Core statistics programming : 2003 to 2008

\begin{tabular}{|c|c|c|c|c|}
\hline Operations & Key objectives of operation & Periodicity & Year of survey & Sample size \\
\hline 3 rd RGPH & See project technical specification & Every 10 years & $2002-2003$ & Full population \\
\hline Employment survey & $\begin{array}{l}\text { Household employment } \\
\text { Unemployment and underemployment } \\
\text { Income source and distribution }\end{array}$ & Annual & 2003 & 12000 households \\
\hline Informal sector survey & $\begin{array}{l}\text { Sector contribution to GDP } \\
\text { Revenues from sector assets } \\
\text { Formal/informal sectors linkages }\end{array}$ & Five years & 2003 & 6000 Households \\
\hline $\begin{array}{l}\text { Survey on the monitoring of public } \\
\text { expenditure in education and health }\end{array}$ & $\begin{array}{l}\text { Budget allocation system in education and health. } \\
\text { Utilization system (schooling, medical, enrollment) of revenues from health and } \\
\text { school facilities. } \\
\text { User's satisfaction with public service delivery in health and education }\end{array}$ & Five years & 2003 & $\begin{array}{l}\text { Administrations } \\
\text { Users }\end{array}$ \\
\hline $\begin{array}{l}\text { Household expenditure and standard of } \\
\text { living survey }\end{array}$ & $\begin{array}{l}\text { Updating the poverty profile } \\
\text { Relationship between types of poverty monitoring the impact of policies }\end{array}$ & Five years & 2006 & 8000 Households \\
\hline $3^{\text {rd }}$ Population and health survey & $\begin{array}{l}\text { Health status (morbidity, prevalence, immunization monitoring, birth rate) } \\
\text { HIV/AIDS prevalence } \\
\text { Nutritional status of children } \\
\text { Reproductive health } \\
\text { Analysis of supply/demand equilibrium in services }\end{array}$ & Five years & 2003 & 8000 Households \\
\hline Educational survey & $\begin{array}{l}\text { Accessibility and affordability } \\
\text { Measure effectiveness of education } \\
\text { Analysis of supply/demand equilibrium in education }\end{array}$ & Five years & 2004 & 8000 Households \\
\hline Agriculture survey & $\begin{array}{l}\text { Production and yield } \\
\text { Use of fertilizers and inputs } \\
\text { Access to land and marketing }\end{array}$ & Five years & 2005 & $\begin{array}{l}14000 \text { Households } \\
\text { farmers }\end{array}$ \\
\hline Multiples indicators survey (MICS) & $\begin{array}{l}\text { Characteristics of vulnerable groups } \\
\text { Identifying their needs } \\
\text { Targeting actions toward improving their standards of living }\end{array}$ & Five years & 2006 & 6000 Households \\
\hline
\end{tabular}




\section{Annex 3.1 Major stages of PRSP elaboration and participatory process}

The participatory process of the national poverty reduction strategy was launched in January 2000 in Kribi during a training seminar which brought together representatives of government and semigovernment administrations, universities, NGOs, different faiths, and donors. This seminar was a first opportunity to exchange ideas regarding the determinants of poverty in Cameroon as well as a discussion of the strategic approaches that can guarantee a reduction of this curse. Concurrently, it allowed participants to make an in-depth analysis of the organizational methodologies of these participatory base-population consultations?

In March of 2000, multi-disciplinary teams working in close collaboration with administrative authorities and NGOs with solid on-the-ground presence were sent to the country's 10 provinces in order to identify 205 groups of people with whom to consult, of which women and young people formed the majority.

The first phase of the participatory consultation was conducted from March 31 to April 10, 2000. It took place in all of the country's 58 regions, and included 203 of the targeted groups representing all sectors of civil society as well as all of the "living strength" of the country. Overall, almost 10,000 people freely expressed themselves during the above mentioned consultations, and of these, $40 \%$ were women. These totally open discussions were conducted in the presence of IMF, World Bank, and German GTZ observers. These consultations provided a large quantity of very interesting and useful information regarding (i) populations' perception of basic poverty (ii) their analysis of the causes of poverty, and (iii) their suggestions regarding ways to reduce the "curse of poverty".

The first phase of participatory consultations was presented in May of 2000, during a national workshop attended mostly by members of the civil society that had been party to the consultations. This workshop helped to (i) recapitulate analyses and suggestions collected through the consultations, (ii) improve relationships on the ground, and (iii) identify focal ideas to be elaborated in the interim Poverty Reduction Strategy Paper(I-PRSP).

In October 2000, the presentation of the I-PRSP during a workshop helped (i) strengthen private sector and civil society contributions to the drafting of the full PRSP, and (ii) establish the basis for an active participation of these key players in monitoring the implementation of the strategy.

In August 2001, and in the context of finalizing the participatory PRSP, the government delivered more than 500 copies of the I-PRSP to all of the representatives at the National Assembly, to provincial Governors, to region Commissioners, and to Deans of public and private universities in order to solicit their suggestions for improvement as well as those of students, teachers, and researchers interested in economic and social issues.

A pilot consultation conducted in October 2001 at Maroua allowed for the testing and fine-tuning of the steps chosen by the government to conduct the second phase of the participatory consultations.

In January 2002, some one hundred facilitators and rapporteurs, chosen from civil society and public administrations and divided into 16 groups, were brought-up-to-speed with the methodology of consultations in order to launch a second round of participatory consultations.

During the same month, the second round of participatory consultations took place in all of Cameroon's 58 regions, enlisting the participation of 6000 people from all walks of life. These consultations allowed people, and notably the Poor to (i) completely specify and prioritize the key elements of the poverty profile at the regional levels as they appeared in the participatory consultations of March/April 2000, (ii) to inform concerned populations of the poverty reduction strategies 
considered within the PPTE Initiative, (iii) gather suggestions to improve the I-PRSP as well as poverty reduction project ideas, and (iv) to share with regional participants preliminary ideas regarding the configuration and the functioning of the National Poverty Reduction Network.

The results of the second round of participatory consultations were made public in April 2002, during a national "feedback" seminar. This meeting helped to (i) ameliorate consultation relationships in the field, (ii) identify key priorities to better focus the I-DPSR, (iii) select poverty reduction policy and monitoring/evaluation actions and indicators, and (iv) go deeper in debating subjects such as people's mentalities, the role of the state, and the information and communication gap.

An initial retreat was organized in Limbé from June 26 to July 7, 2002 to draft the full PRSP. Multidisciplinary teams made up of national and international experts working in the public sector, and civil society experts took part in this retreat. The output from their work was reviewed for consistency by the Comité Technique de Suivi des Programmes Economiques, economic programs monitoring technical Committee (CTS).

From November 28 to December 3, 2002, a second drafting retreat took place in Kribi with the participation of public sector administrations and national consultants which had worked together with the government throughout the PRSP exercise. This second retreat was an opportunity to (i) examine the PRSP macro-economic and budgetary calibration projects, (ii) align the objectives of the SPRS with the proposed macro-economic and budgetary calibrations previously proposed, and (iii) take into account the first observations made by some partners on the PRSP project which took place in Limbé.

From December 6 to December 10, 2002, the government circulated the PRSP project follow-up developed at the Kribi retreat to civil society representatives, private sector economic players, donors, and public administrations, and it solicited their observations and suggestions in order to enhance this document. Most of the outputs of this very useful and informative exchange were taken into account in order to further strengthen the final PRSP.

From December 16 to December 19, 2002, participants from civil society organized a brainstorming workshop to formulate their contribution to the enhancement the relevance of the PRSP project. This workshop helped participants (i) understand the elaboration process and implementation of the PRSP, (ii) analyze the role and place of civil society in the implementation of the I-PRSP, (iii) design a presentation strategy to inform other members of civil society, the government and the donors on the outputs of the workshop, and (iv) elaborate a strategy to take into consideration civil society as a partner in the organization of the PRSP.

On December 27, 2002, a seminar designed to broaden the exchange of views was attended by civil society's representatives and private sector operators. This seminar helped collect observations emanating from key actors in poverty reduction on the PRSP project to bring forth final improvements. 


\section{Annex 3.2 Definition of poverty by different population groups during the participatory consultations}

To faithfully convey grassroots concerns, efforts have been made to report the following information as closely as they were expressed during the participatory consultations of March/April 2000 and January 2002 in the 10 provinces.

\section{ADAMAOUA}

- A poor person is perceived as someone lacking the resources to cover basic needs: children's education, food, health, clothes, housing, and mobility (financial poverty).

- A person with financial means, but one who abstains from using them to cover personal needs and those of his relatives (psychological poverty).

- Social exclusion is another perception of poverty.

- Poverty is begging.

- It is lack of training for adults.

- Poverty is a lack of industries.

- Poverty is also associated with the difficulty of access to modern means of communication (telephone, radios, and television), healthcare, drinkable water, and electricity.

- Poverty is associated with a lack of decent housing.

\section{CENTER}

- Poverty is perceived as a lack of means which prevents the individual, the local group, and the state from providing for basic needs.

- It is a dependency phenomenon.

- Poverty is a living reality that affects the essence of human life. It can have a moral nature, psychological, intellectual, environmental, material or cultural one.

- It is perceived as the lack of means to meet basic needs and of social well-being.

- It is conservative mentalities aggravated double-fold by a lack of initiative and innovation drive.

- Poverty is an inadequacy between aspirations and available financial means to satisfy them.

- It is also financial and material penury due to the economic crisis.

- Poverty is also a state of mind, that is, ignorance of current potential capital.

- It is the inability to have the minimum to feel one's self, have access to healthcare, obtaining an education, clean drinking water, adequate housing, clothing.

\section{EAST}

- Poverty is perceived as a state of destitution preventing an individual or a society from satisfying basic needs as well as those necessary to development.

- It is also the increasing difficulty for population to move around and market their agricultural products.

- Poverty is a difficult access to sources of drinkable water, lack of electricity, and lack of necessity items.

- Poverty is the degradation of people's habitat, health and educational infrastructures in rural regions.

- Poverty is also malnutrition, prostitution, insecurity, selfishness, mendacity, and lack of financial resources.

- It is early marriage, early and unwanted pregnancies.

- Poverty is a lack of means of communication.

- One is poor when one has only rudimentary agricultural equipment.

- Poverty is fate.

- It is laziness, immobility of those that cannot take care of themselves as they are unable to manage their own resources.

- Poverty is also social exclusion.

- Poverty is being overly landlocked. 
- Poverty is also the state of someone who experiences social servitude and also one who is from a dysfunctional family.

- It is the inability to think through and find solutions to problems.

- Poverty is misery in every aspect of life and loss of dignity.

\section{EXTREME -NORTH}

- A person is poor when he/she does not have fish to prepare with the sauce.

- We are poor when we do not have soap to clean the clothes.

- Poverty is when the lack of roads prevents engaging into commercial activity.

- Poverty is schools being far away, not dispensing an effective and real education, a lack of secondary schools.

- Poverty is also when teachers are penniless and parents must help pay the teachers' wages and build classrooms.

- It is also the lack of accountability among healthcare and education workers who either do their job badly or not at all.

- We are poor when we live in an environment lacking paved roads and pharmacies.

- A poor person is one whose land is poor and produces little.

- Poverty is the state of conflict between farmers and ranchers.

- It is also the lack of activities and mobility.

- Poverty is the feeling of powerlessness that arises from devastation caused by elephants trampling one's farm.

- Poverty is the lack of roads, water supply, and communication means.

- Poverty is the exorbitant hike in the price of merchandise.

- A person is poor when he/she is unable to cater to one's basic needs such food, shelter, healthcare for one's self and one's children, or having a hurricane light.

- One is poor when one does not have any transportation means (bicycle).

- A poor person is one who travels long distances to get water.

- A poor person is one who is ashamed.

- A poor person is one who cannot even pay for salt at 25 Francs CFA.

- Poverty is also perceived as a lack of healthcare facilities in villages.

- Poverty is lack of money.

- Poverty is the absence of means of subsistence.

- Poverty is the lack of a bed in which to sleep.

\section{LITTORAL}

- Poverty is the lack of material and financial resources necessary to meet basic needs of individuals or families (food, shelter, healthcare, and schooling for children).

- Poverty is ignoring the presence and powers of God.

- Poverty is the lack of happiness in one's existence.

- Poverty is also defined as belief in a fatalistic destiny (resignation).

- Poverty is laziness.

- Poverty is ill-health.

- Poverty is a permanent state of feeling inferior and being ashamed.

- Poverty is distress.

- Poverty is lack of hope and lack of jobs.

- Poverty is mental feebleness.

- Poverty is lack of wage-earning activities.

- Poverty is the uncertainty of tomorrow.

- Poverty is the lack of knowledge.

- Poverty is a smaller difference between the man and the animal.

- Poverty is a lack of roads, and landlocked villages.

- Poverty is the lack of agricultural inputs and of extension services for villagers. 
- Poverty is inconsistency between training and employment. It is the lack of opportunities in the rest of the world.

- Poverty is the inability of an individual to manage his/her basic necessities, the inability to plan for anything because nothing seems to be stable.

- Poverty is a lack of credit structures.

- Poverty is also perceived as the tally of deceptions the state inflicts on its back country citizens.

\section{NORTH}

- The poor is one who cannot cater to basic needs: food, healthcare, children's education. It is the person living in inadequate housing conditions (shabby construction materials, uninhabitable space).

- It is also one without a job.

- The Poor is one unable to read or write.

- The Poor is the one who is resigned to his/her fate.

- The Poor is the one who is poorly dressed.

- The Poor is the one who suffers physically and mentally.

- The Poor is one who does not have land to cultivate.

- The Poor is the one who does not have a stable family.

- The Poor is the one who can find a way out of difficulties.

- The Poor is the one who resort to usury in order to survive.

- The Poor is the one who begs.

- The Poor is the one unable to obtain farming equipment.

- The Poor is the one who lives in a landlocked place.

- The Poor is one that lives off of the sale of millet and beer.

- The Poor is the one who is lazy.

- The Poor is one who is excluded and marginalized by society.

- The Poor is the one who lives insecurely.

- The Poor is the one who lacks information.

- The Poor is the one who cannot afford to have free time and professional training.

- The Poor is the one who cannot obtain a credit or save.

- The Poor is the one who cannot lease an agricultural field.

- The Poor is the one who cannot buy fertilizers.

- $\quad$ The Poor is the illiterate.

\section{NORTHWEST}

- The Poor is a frustrated person.

- The Poor is a beggar.

- The Poor is a dependent person.

- The Poor is a lazy person.

- The Poor is with an inadequate education.

- The Poor is one who cannot offer a dowry to a woman.

- The Poor is the one who is incapable of cater to one's basic needs.

- The Poor is the one who is not a COOPEC member.

- The Poor is the one with no initiative.

- The Poor is one who is incapable of selling his/her agricultural products.

- The Poor is one who weakened physically and morally.

- The Poor is the one who does not believe in God.

- The Poor is one without any financial means.

- The Poor is one who does not have enough arable land.

- The Poor is one with a big family.

- Poverty is the state of insecurity.

- The Poor is the one without access to sources of credit and social infrastructures.

- The Poor is one who is under-employed. 
- The Poor is one who is idle (unemployed).

- The Poor is a person lacking happiness.

\section{WEST}

- Poverty is defined as an inability to access healthcare services and to secure the education and training of one's children.

- Poverty is the inability to get the basic foodstuff.

- It is also the erosion of family life.

- Poverty is the lack of access to justice.

- Poverty is insufficient revenue.

- Poverty is a degradation of morality.

- Poverty is insecurity and growing crime.

- Poverty is the degradation of the living environment and promiscuity.

- Poverty is the increase in death rates.

- Poverty is difficult access to land for cultivation purposes.

- Poverty is difficulty marketing of products.

- Poverty is not having a voice.

- Poverty is the lack of housing.

- Poverty is lack of roads that lead to the market, the school, or the hospital.

- Poverty is a lack of transportation means.

- Poverty is lack of guaranties to obtain credit.

\section{SOUTH}

- Poverty is defined as a lack of money, housing, food, material means, medication, organization, and employment.

- Poverty is a difficulty to provide for the children's schooling needs.

- Poverty is the lack of roads.

- Poverty is the bad state of one's health.

- Poverty is lack of children.

- Poverty is the inability to get treatment.

- Poverty is lack of education, electricity, and drinkable water.

- Poverty is poor nutrition.

- Poverty is being invalid.

- Poverty is the lack of a spouse.

- Poverty is a lack of well-being.

- Poverty is rejection of progress.

- Poverty is characterized by low wages.

- Poverty is the inability to invest.

\section{SOUTHWEST}

- A Poor is one who is unable to procure the strict minimum; housing, clothes, food, and wife.

- The Poor is a person with bad health.

- A Poor is one who is never happy.

- A Poor is a lazy person.

- A Poor is one without income.

- A Poor is one who cannot take care of him/herself nor even to build upon his/her potential.

- The Poor is also that person who is ashamed.

- The Poor is one who does not have access to land, to credit, drinkable water, and electricity.

- A Poor is one who does not love peace.

- A Poor is one who is a victim of political manipulation.

- The Poor is the one who has many children.

- The Poor is the one who cannot have three meals a day. 


\section{Annex 3.3 Poverty reduction actions and strategies derived from the participatory consultations}

Based on the diagnostic test conducted in order to describe and characterize poverty, the populations have proposed solutions that were meant to contribute to the reduction of poverty. Most of the proposed actions have already been included in the state budget and even in the financing of the PPTE in the intermediary period 2000-2003. They address the following strategic priorities: (i) transportation and communication infrastructures, (ii) education and training, (iii) health and HIV/AIDS, (iv) other social sectors, (v) agriculture, fishing, and breeding, (vi) environment and forest, (vii) drinking water, hydraulics, and availability of electricity in rural areas, (viii) oversight and credit structures, (ix) employment/revenue, (x) trade and industry, (xi) governance, and (xii) borders policy.

\section{Transport and communication infrastructures}

The populations suggested opening up all regions and ensuring (i) the rehabilitation and maintenance of the existing road network, (ii) the paving of the major national and inter-provincial roads, (iii) the multiplication and maintenance of rural tracks in order to guarantee the movement of agricultural and pastoral products toward centers of exchange and most notably the marketplace, (iv) national coverage in the area of communication especially in border areas (radio, television, and others), and (v) availability and promotion of technologies of information and communication (TIC).

\section{Education and training}

The main recommendations pertain to (i) equipment, construction, and rehabilitation of primary and secondary schools, (ii) the construction of new universities adjusted to the regions' realities, (iii) reinforcing educational staff qualitatively and quantitatively followed by inciting guidance measures (iv) a revision of primary, secondary, and university programs in order to adapt training to employment needs and to stress civic, technical, and moral education, and (v) the expansion of teaching to poor and marginalized sectors of society.

\section{Health and HIV/AIDS}

Grassroots suggestions concerning this sector of vital importance for the well-being of populations covered (i) equipment, construction, and rehabilitation of healthcare and social structures throughout the country, (ii) the strengthening and valorization of healthcare personnel both qualitatively and quantitatively, (iii) treatment of victims of HIV/AIDS, (iv) intensification of the fight against HIV/AIDS, Malaria and/or other endemic illnesses such as meningitis and hepatitis (viral), and (v) the stocking of medical facilities with necessary generic medicines which are affordable to all levels of society.

\section{Other social sectors}

Interviewed populations proposed (i) multiplying training and social promotion centers to the benefit of street kids, young girls, and the disabled, (ii) developing and applying generalized educational programs and fighting against the use of illegal drugs and alcohol, (iii) elaborating and implementing a realistic integration and supervision program for vulnerable and/or marginalized groups, (iv) encouraging and supervision, through NGOs and women's associations, (v) expanding literacy programs to all population levels, and (vi) making issuance of birth certificates automatic and mandatory.

\section{Agriculture, fishery, and livestock}

In this important sector of the national economy, recommendations revolved around (i) designing and implementing an integrated rural development policy, (ii) returning to State's control the major liberalized subsectors, (iii) promoting research on diversification and transformation of agro-pastoral products, (iv) creating transformation and preservation units for products in order to guarantee their transfer from peri-urban areas to large cities' markets, (v) reinforcing the supervision training of farmers, ranchers, and artisans, (vi)modernizing methods used in cultivation, fishing, and production of handicrafts, (vii) facilitating access to machinery and financing of agro-pastoral and handicraft 
activity, (viii) opening and maintaining agriculture outputs collection roads, (ix) supporting farmers' groups and specifically women working in agriculture, ranching, and handicrafts, and (x) supporting locust control and fighting high sea piracy.

\section{Environment and forest}

People's suggestions included (i) protecting the environment of neighboring populations, (ii) expanding and managing the forests using the zoning plan of the Ministry of the Environment and Forestry (MINEF), (iii) implementing a communal forestry training program for the concerned populations, (iv) reviving the "Green Sahel" operation, and (v) intensifying and extending urban improvement projects across the country.

\section{Hydraulics and rural access to electricity}

Proposals for solutions to the problems linked to hydraulics and rural access to electricity covered (i) the extension of hydraulic and rural access to electricity programs, (ii) the densification of the electric grid through the construction of power generating micro-dams and (iii) and the planning and development of drinkable water points throughout the territory.

\section{Oversight and credit structures}

The populations has solicited (i) the creation of additional advisory and assistance structures, (ii) the development of saving and credit structures to help finance development activities, and (iii) the establishing of a development bank and of a micro-financing structure for rural areas economic activities.

\section{Employment/income}

In order to develop a good policy of income redistribution backed by significant actions that guarantee full employment, the populations suggested (i) limiting out migration by multiplying income generating activities in rural areas, (ii) giving the National Employment Fund (FNE) the full role it deserves in the employment sector, (iii) increasing civil servant salaries, (iv) encouraging trade through an incentive fiscal policy, (v) foster the creation and growth of SME/SMIs through better access to credit endorsed by the government, and (vi) reevaluate the recruitment policy in the public sector by taking into consideration the regional specificities, restrictions imposed on higher education degree holders due to the economic crisis who find themselves unable to obtain public sector job because of their advanced age.

\section{Commerce and industry}

The development of a real industrial and commercial network seemed to be an urgent need. To that end, populations have proposed (i) establishing an adequate industrial policy aimed at extracting existing mining and forest resources through the creation of factories which could constitute a core for development areas in different regions of the country, and (ii) developing the tourism industry.

\section{Borders policy}

The consulted populations wished for the state to establish a real development policy in border areas because of the screening role they play with neighboring countries. To that end, they have proposed that transportation infrastructures (in these areas) be further developed to make Cameroonian products more competitive.

\section{Governance}

In terms of good governance, populations have proposed (i) restoring the government's authority, (ii) pursuing the decentralization/deconcentration procedures of public management, (iii) intensifying anticorruption campaigns, (iv) reinstating civic education in school programs, (v) providing more resources to law enforcement agencies to be more effective in their combat against insecurity, and (vi) ensuring an even-handed, across the board enforcement of the law without exemptions. 


\section{Annex 4.1 Branches of the Agricultural Sector}

Cameroon's agricultural sector is characterized by abundant and diversified potential, plentiful natural resources (arable land), a favorable geographic position with the port of Douala situated close to subregional markets, human resources and an institutional framework recording steady improvement. With the further construction of roads and highways to the provinces of the East and South, the market for starches, cereals, fruits, and vegetables offers interesting opportunities for short-term and medium-term growth, particularly for bananas/plantains, potatoes, onions, and cassava.

In the face of declining prices for traditional cash crops, recent years have witnessed remarkable performance by food crops - such as corn, cassava, plantains, potatoes, yams, beans, millet, sorghum, etc. However, in spite of this performance, the sector is still failing to achieve its full potential on account of a multitude of constraints associated with the small scale of rural farms (0.5-1 hectare), deficient human resources, difficulties in obtaining inputs, nonexistent or problematic transport infrastructure, the dearth of financing and organization for the sector, and lastly, the lack of an appropriate institutional environment and support services. This leads to very low productivity, as reflected in current yields which represent about one third of potential capacity, depending on market behaviors and the regions involved.

The agricultural development strategy is designed to identify and promote those branches of the agricultural sector offering the greatest potential, based on the 5 major agro-ecological subdivisions of Cameroon as indicated below:

- For the monomodal forest zone, i.e., the provinces of the coast and the South-West: bananas, plantains, palm oil, ginger, and pepper;

- For the bimodal forest zone, i.e., the provinces of the Center, South, and East: cassava, corn, palm oil, and pineapple;

- For the zone of the high plateaus in the West and North-West: corn, dry beans, potatoes, organic farming of vegetables (including watermelon);

- For the Sudan-Sahel region: millet/sorghum, cowpeas, onion, sesame;

- For the region of the high savannas in the province of Adamaoua: millet/sorghum, corn, yams, and potatoes.

This strategy focuses on two main objectives: (i) boosting yields; and (ii) enlarging surface areas under cultivation. Yields will be boosted by measures to facilitate farmers' access to inputs (plant material, fertilizers, plant-health products), crop cultivation techniques, equipment and material, harvesting, storage, and processing facilities, as well as marketing arrangements (information, infrastructure, etc.). Depending upon the regions and market patterns, cultivated surface areas will be increased, either by scaling back unprofitable and lower-yielding crops which will be effectively abandoned by producers, or by putting new land to work. Various key branches of the agriculture sector are described below.

Cassava. (i) Background: In Cameroon, cassava is one of the primary plant products making the largest contribution to feeding the population. Production is estimated at around 2 million tonnes. Cassava cultivation is a task performed primarily by women, and the same goes for 
the associated small-scale processing operations. Farms are mainly on a small scale, and frequently combine cassava with other crops. The high yields are of the order of 14.5 tonnes per hectare. Cassava production predominates in the Southern zone, with the main producing regions being the Center, the coast, the East, and Adamaoua; these account for over 70 percent of national output. Per capita consumption is estimated at 120 kilograms per year; consumption is largest in producing areas. (ii) Constraints: Efforts to expand cassava production are hampered by the high costs of marketing, intrusive road and highway checks, difficulties in harvesting, the lack of producer information on the price of final markets, the dearth of suitable equipment for processing, and the inaccessibility of a number of production areas, especially in the rainy season. (iii) Strategy: A specific program will be prepared for cassava in light of its importance to society and the economy, with the aim of deriving maximum benefit from the various initiatives in progress (one third of production goes into processing). In addition to meeting traditional requirements for fresh and processed products, this program will take account of subregional demand as well as the national industry's needs for animal feed and starch production. The measures to improve marketing will consist of encouraging the formation of networks for group operation (networking) as well as for collective small-scale processing activities, enhancing the network of service roads, developing mechanization suitable for small-scale processing, while implementing an efficient information system.

Potatoes. (i) Background: Potato production is estimated at 160,000 tonnes at present; this figure has virtually doubled since 1995 . Yield per hectare is 20 tonnes, falling short of agronomic potential, which is approximately 30 tonnes/hectare. Production is concentrated in the provinces of the West, the North-West, and more recently the province of the Far North. The flows of exports intended for Gabon and Equatorial Guinea are estimated at 25,000 tonnes. Per capita consumption is estimated at $11 \mathrm{~kg} /$ year, with an upward trend in the regions of the Great West and the Coast. (ii) Constraints: The principal constraints standing in the way of successful potato production are the problems with seeds, the administrative constraints (plant-health controls that are ineffective but mandatory and burdensome; intrusive road and highway checks), the poor condition of rural roads, the unreliability of statistical data, and the failure to promote exports. (iii) Actions: The production/distribution of high-quality seeds, the implementation of a collection system, the maintenance of rural roads, capacity-building for farmers with a focus on networking, and export promotion would improve the current levels of production and consumption.

Plantains. (i) Background: Plaintains are produced throughout the Southern part of Cameroon, particularly in the provinces of the South-West, the West, the Center, the South, and East. Plantain production employs about 600,000 farmers who each year produce 1.2 to 1.5 million tonnes, i.e., an average production of 2.3 tonnes per agricultural holding/farm. This comparison highlights the leading role played by smallholdings (farms with less than one hectare planted with plantains). Yields are estimated at about 4 to 7 tonnes per hectare, well below the agronomic potential which is of the order of 30 tonnes/hectare. Exports, primarily headed for Equatorial Guinea and Gabon, are in the range of 10,000 and 20,000 tonnes. Per capita consumption is estimated at approximately $66 \mathrm{~kg}$ in Yaoundé, $108 \mathrm{~kg}$ in Douala. Consumption in rural areas is in the range of 70 to $125 \mathrm{~kg}$ in the provinces of the South; it is practically nil in the Northern part. (ii) Constraints: The constraints on plantain production are primarily attributable to the costs of marketing (transport, intermediation, profit margins) which are high and liable to increase further; they are also due to the shortage of good-quality rejects, as well as the absence of processing development and information systems. (iii) Actions: Requirements for improving current production and consumption levels include 
the dissemination of improved varieties and techniques for ensuring that plant material is rapidly multiplied by producers, achieving improvements in cultivation practices, an increased reliance on transportation in baskets rather than in bulk, organization for the plantain sector, training and information for producers, as well as export promotion.

Corn. (i) Background: Corn production is found in all provinces of Cameroon and contributes CFAF 5,605 billion to GDP. Production is estimated at 810,000 tonnes with a yield of 2 tonnes/hectare, falling short of agronomic potential which is of the order of 5 tonnes/hectare. Nine percent of this output is used in agro-industry to manufacture grits and feedingstuffs, 4 percent is exported to Gabon, Congo, and Chad, with the remainder divided up among own-consumption and marketing. Per capita consumption of corn is esimated at $8 \mathrm{~kg}$ in Yaoundé, somewhat less in Douala; it is in the range of 80-100 kg in the North, and $30-50 \mathrm{~kg}$ in the West and North-West. (ii) Constraints: Efforts to expand corn production are essentially hampered by breakdowns in the marketing system, the high costs of product collection, and the lack of storage facilities or high-quality seeds. (iii) Actions: Actions will focus primarily on (a) fertility management and soil protection in order to enhance productivity and ensure the sustainability of cultivation systems, the production of quality seeds, the development of animal haulage, and improvements to village storage methods; (b) development of contractual relationships between producers and processing units; and (c) enhancing the productivity of post-production operations to facilitate penetration into urban markets.

Millet and sorghum. Efforts will focus on: (i) intensifying efforts to disseminate the technical pathways already identified through research, (ii) developing anti-erosion technologies and the use of organic soil conditioners in the context of land management programs; (iii) pushing ahead with the implementation of village storage warehouses in order to minimize post-harvest losses; (iv) boosting efforts to upgrade production; and (v) establishing a seed reserve. These various measures will all help to strengthen the food security program for the Sudan-Sahel zone. 


\section{Annex 4.2 Key branches of the industrial sector}

The studies envisaged in the priority action plan will highlight the potential capacities of the various branches of the sector, as well as the steps that must be taken to fulfill that potential. A number of branches are discussed below, although this list is by no means comprehensive.

\section{Poultry industries}

The poultry industry is a promising sector from the following standpoints:

- Pro-poor growth, with a major spinoff effect on agricultural production (corn) and industrial production (grandnut cake, cotton cake): National and regional demand represent untapped potential. If average per capita consumption were to increase from 1.3 $\mathrm{kg}$ (2000) to $5 \mathrm{~kg}$ (a level comparable to Côte d'Ivoire), actual production of chickens and feedingstuffs would be $5 \frac{1}{2}$ times and $4 \frac{1}{2}$ times greater than their current production levels.

- Nutrition: Chicken owes its global popularity to its affordability and the fact that it is taboo-free in nearly all cultures. In Cameroon, however, the poultry industry accounts for only a negligible portion of Cameroonian households' consumption of animal protein. This is attributable to the relatively high prices of chicken meat, as the demand for animal protein is very high relative to prices and incomes. An increase in factor productivity would make it possible to lower the cost price of poultry and to turn it into an inexpensive protein source that is affordable for households.

- Self-employment: This sector offers opportunities for microenterprises, including women. Barriers to entry are manageable, the required investment is modest, and the production cycle is short ( 2 months for chicken for slaughter).

The major challenge facing the poultry sector is how to lower cost prices while maximizing factor productivity:

- The high cost of chickenfeed: Cameroon must go abroad to obtain 60 percent of its corn, the price of which is high and fluctuating.

- The low yield of production factors in particular, the duration of growth, the weight, the egg-laying yield, death rate, preventive health issues, etc.

- The lack of specialization: one single operator produces chickens, eggs, feedingstuffs, etc.

- A lack of familiarity with the latest techniques in stockbreeding and management.

- A lack of synergy: farms operate in isolation from one another.

- The absence of industrial slaughterhouses (just a handful of small-scale poultry slaughtering facilities), and the lack of storage resources, which means that small-scale poultry farmers are obliged to handle the marketing for their farms themselves. This pushes up poultry production costs (problems in disposing of produce lengthen production cycles).

- The difficulties in gaining access to financing, particularly since the liquidation of FOGAPE.

In designing a coordinated strategy focusing on the following areas:

- Create conditions for the distribution and collection of corn. 
- Develop an agricultural subcluster system comprising 3 operators: the poultry producer, the feedingstuffs producer, and the slaughtering/packaging operators around which breeders will gravitate.

- Reduce costs by improving the quality of feedingstuffs and poultry.

- Minimize building construction costs through the use of local materials.

- Ensure vocational training and retraining to improve performance in the areas of technology and poultry management.

\section{Textiles and clothes-making}

\section{Background:}

Cotton production is handled by approximately 300,000 farmers located in the Northern provinces of Cameroon. They produce annually about 80,000 tonnes of cotton fibre, under the aegis of the Cotton Development Company (Société de Développement du Coton (SODECOTON)). Over 95 percent of cotton fiber is currently exported. That figure reflects a very low rate of processing for local cotton in comparison with the potential value creation in the areas of spinning and weaving, and clothes-making.

Spinning is carried out by private firms, in particular, CICAM (over 90 percent of local production) and NOUFIL, which use cotton fiber from SODECOTON and imported synthetic fibers. Weaving is handled by CICAM, SOLICAM, SICABO, and SINCATEX, which produce a wide range of fabrics for various purposes (loincloths, fabrics for clothing, sheets, upholstery, etc.). This activity notwithstanding, this market segment still holds considerable growth potential in regard to the design and production of brand-name fabrics for various uses, intended for local and export markets.

Clothes-making encompasses numerous small-scale and micro-enterprises (tailors, stylists, dressmakers) whose products (menswear, clothes for women and children)-which are of varying quality - are mainly intended for the local market. If the upstream activities in this industry have always enjoyed the support of the government authorities (viz. the activities of SODECOTON), this has not always been the case with its downstream activities, spinning, weaving, and clothes-making, which are chiefly conducted by private transactors.

The main constraints facing clothes-making are associated with: (i) the low degree of synergy between designers and distributers and key markets; (ii) the fact that production is still organized on a non-industrial scale, with negligible specialization in the conduct of operations; (iii) insufficient human resources (vocational training programs are not standardized); (iv) a low level of synergy between producers of fabrics, industrial clothesfacilities, and designers; (v) the lack of an institutional framework to support small-scale enterprises and microenterprises engaged in clothes-making, of a kind that could remedy their deficiencies by organizing training activities in the areas of design, clothes-making techniques, and methods with respect ot production, management, and marketing, etc.

A strategy for developing the downstream activities of the textiles/clothes-making sector in Cameroon would enable the firms in this sector to become more competitive (through the strengthening of synergies between markets and suppliers, support for vocational training, exchanges of experience, enhancement of manufacturing capacities: manufacturing methods, quality control, standards, management, etc.). Businesses could then utilize their competitive advantages (cultural heritage, creativity, thèque fabric, etc.) on local, regional, and 
international markets (European, African, and Latin American customers). Yaoundé could become a centerpiece for fashion in Africa.

In closing, the design and implementation of a targetted strategy, coupled with appropriate incentives, should help mobilize resources and rally stakeholders (private and public) around the textiles and clothes-making industry and thereby enable this sector to achieve its potential. 


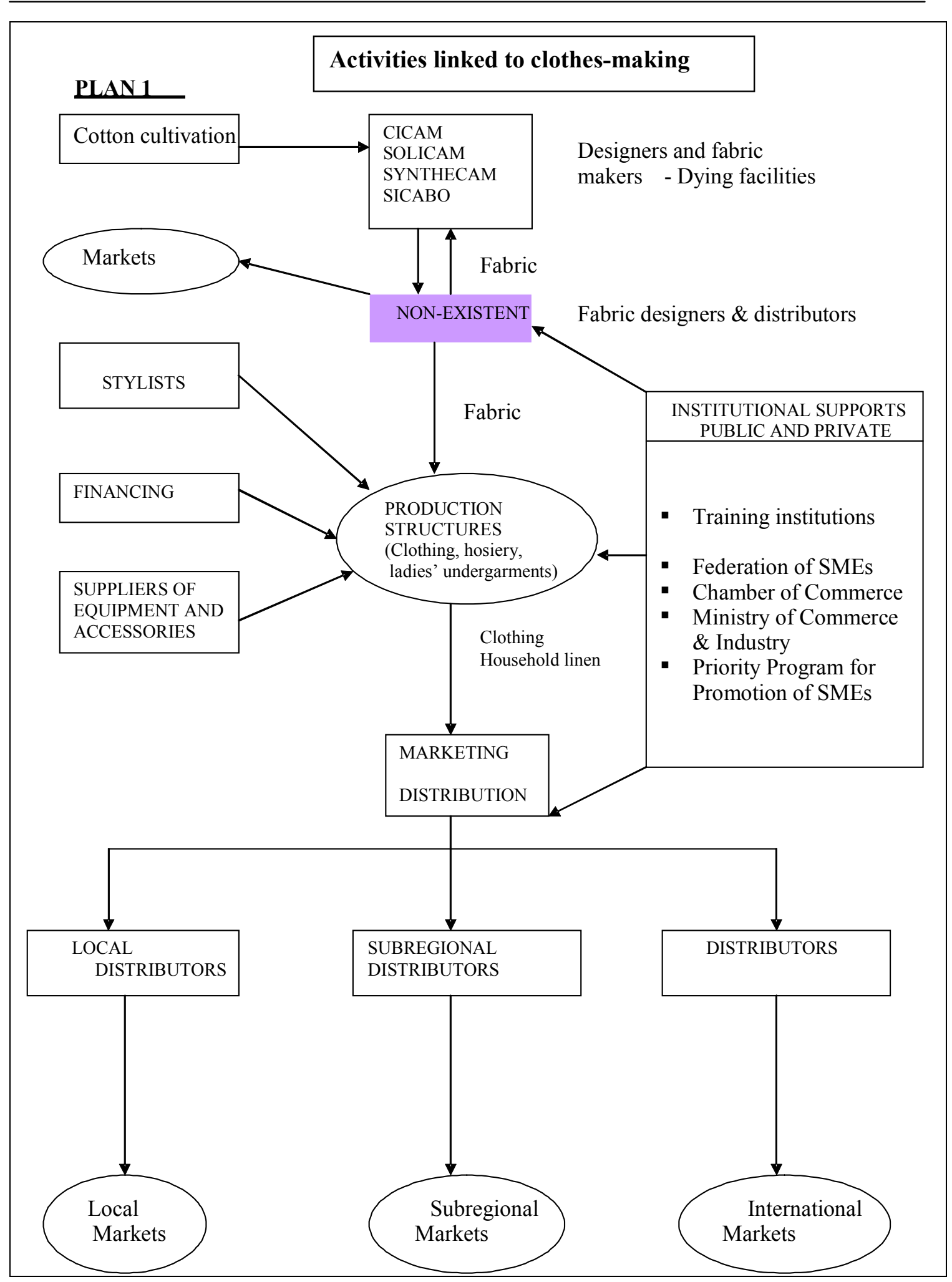




\section{Tertiary processing of wood}

The Cameroonian forest, which covers 24.5 million hectares, plays host to a remarkable variety of flora and fauna. Of the 300 species identified, operations focus on approximately 60 species of which the 5 main ones account for 70 percent of the total production estimated by loggers: ayous/obeche, azobe, frake, iroko. These figures adequately demonstrate the manner in which forest resources can be harnessed. The forestry sector has considerably improved its share of national GDP, moving from 4.3 percent in 1992 to virtually 6 percent in 1997.

The upstream activities in the forest sector include the first and second stages of processing which account for the bulk of the resources and the major loggers. The products in these categories, roundwood and sawnwood, are nearly all exported to Europe and Asia. Cameroon has approximately 70 processing units including about 60 sawmills, 5 rotary cutting units, 1 slicing plant and 1 "matchstick" manufacturing plant. Still focusing on upstream activities, there is a third category consisting of small-scale loggers (mainly domestic) who hold 48 percent of the surface areas under license and produce on average 10 percent of the total volume. Their products are intended for the local market.

The sector's downstream potential: While the sector's upstream activities are the focus of forestry reforms, the sector's downstream activities - and in particular the tertiary processing of wood-have not received specific support, in spite of their enormous potential for value added and job creation. In fact, the value of 1 cubic meter of furniture is equivalent to about 7 to 8 times the value of a cubic meter of logs, or 2 to 3 times the value of a cubic meter of sawnwood. This shows how value is lost when the Cameroonian lumber industry continues to focus on the first and second stages of processing. This justifies any policies designed to extract further benefits from lumber resources through the development of more finished products. Furthermore, this segment includes a substantial contingent of small- and mediumscale enterprises and microenterprises producing furniture and other cabinetmaking products for the local market, albeit without any special support. In addition to secondary species which are not taken into account in current forestry operations, other wood-type materials such as rattan and bamboo (major raw materials in Cameroonian craftsmanship) ought to find viable export outlets. But achieving this objective will require developing appropriate supports for small-scale and medium-scale enterprises and microenterprises in this segment of the lumber sector in the areas of design, technology, organization of production, financing, and marketing.

The main constraint on developing the third stage of processing of wood is the lack of a suitable institutional framework for addressing the problems facing enterprises in this sector, particularly with respect to training, access to raw materials, marketing, technologies, financing and equipment. The planned research to prepare a strategy for developing the tertiary processing of wood must identify optimal conditions for capacity-building in this segment of the lumber industry.

\section{Fisheries}

Background: The fishery sector in Cameroon is organized around 3 branches of activity: industrial fishing, artisanal fishing (offshore and inshore), and aquaculture. Cameroon's domestic fishery production is 120,000 tonnes of fish per year which is divided up as follows: 50,000 tonnes for inshore fishing, 60,000 tonnes for artisanal offshore fishing, 10,000 for industrial fishing (including 500-1,000 tonnes of shrimp), with 50 tonnes for aquaculture. 
Potential fishery resources are estimated at approximately 155,000 tonnes per year, including 65,000 tonnes for offshore fishing, 75,000 tonnes for inland waterway fishing, and 20,000 tonnes for aquaculture. This potential is not fulfilled because of the constraints associated with each type of fishing. Artisanal fishing (inshore and offshore), which is handled exclusively by rural communities, employs over 200,000 individuals, including 65,000 persons employed directly and 135,000 persons employed indirectly (sales of fresh fish, processing, construction and repair of fishing gear). The current needs of the population currently stand at around 230,000 tonnes of fish per year, i.e., a shortfall of 110,000 tonnes, which is partly met by imports $(60,000-80,000$ tonnes of fish per year). Per capita consumption of fishery products is estimated at around $16 \mathrm{~kg}$ per year, making fish the primary source of animal protein. Thirteen companies handle the bulk of production in the industrial fishing segment, with a fleet (year 2000) estimated at 77 trawlers and shrimpers.

Constraints: Industrial fishing has been in the doldrums due to the fact that Cameroonian vessels have found it difficult to gain access to the fish-rich waters of neighboring countries. Further problems have included the absence of an effective and permanent system for the monitoring, control, and surveillance of waters as well as the failure to ensure proper outfitting of the landing areas on fishing vessels, as well as the lack of statistics. Efforts to develop artisanal fishing are hampered by the dearth of transportation infrastructure, which is responsible for losses of the order of 30-35 percent of production-i.e., approximately 33,000 tonnes approximately, which represents over 3 times the amount of industrial output. These constraints are compounded by the difficulties involved in gaining access to financing; the lack of organization among participants in the sector; the absence of a supporting institutional framework or of control/surveillance mechanisms. Aquaculture suffers from such constraints as the interdependence of the various links in the chain (fish-farming station or rearing center, fish population monitor, fish farmer), notably with respect to the supply of young fish; the disregard for technological or management standards; the sluggishness of administrative procedures; constraints on access to credit; and low yields.

Potential: Nonetheless, Cameroon enjoys substantial advantages, including: a shoreline of 360 kilometers with a continental shelf of $14,000 \mathrm{~km} 2$; an "exclusive economic zone" (Zone Economique Exclusive (ZEE)) covering some 25,000 km2, and a total surface area of inland waters amounting to approximately 4,000,000 hectares; continental plains; natural lakes; Lake Chad (800-1,800 km2); artificial lakes created by hydroelectric dams; primary and secondary rivers flowing into four basins (Congo, Atlantic, Niger, and Lake Chad). Artisanal fishing holds the strongest potential, provided that it receives appropriate institutional support in the context of community development projects to help artisanal fisherman gain unimpeded access to adequate fishing equipment and materials. In carrying out diagnostic assessments of sectors offering potential, particularly close attention will be paid to efforts to promote artisanal fisheries, drawing inspiration from the examples of such countries as Senegal and Ghana.

\section{Processing of fruits and vegetables}

Background: Fruit and vegetable production reached nearly 845,000 tonnes in 1999 . Substantial quantities of mango, citrus, pineapple, avocado, papaya, and guava are produced by farmers in most of the Southern provinces. Except for pineapple and papaya, which are intensively farmed in the department of Mungo and in certain districts of the Southern provinces, all the other crops come from orchards distributed over areas reserved for food crops and cash crops, unless they are used as hedges encircling huts. Domestic production is 
dominated by fruits, with about 531,000 tonnes, followed by vegetables representing about 315,000 tonnes. Current per capita consumption is estimated at $19 \mathrm{~kg}$ of fruit (50 percent occurring in the months of July and September) and $17 \mathrm{~kg}$ of vegetables.

Constraints: According to the ILO, annual losses of fruits and vegetables are of the order of $30-50$ percent of overall production. Based on this percentage loss, the pertinent figure is 265,500 tonnes for fruit and 157,500 tonnes for vegetables. According to various assessments, production units in the fruit and vegetable processing industry experience difficulties in obtaining raw materials of the desired volume and quality (the use of recycled packaging is not yet suited to the nature of the products concerned). Further constraints include: the lack of appropriate equipment; a failure to ensure strict compliance with the production standards applicable to agri-business; a family-based approach toward the management of production units; limited storage and warehousing capacity for raw materials and for finished products.

As a country seeking to develop its fruit and vegetable processing industry, Cameroon enjoys a number of advantages, including: agro-ecological diversity; sizable production, albeit with post-harvest losses reflecting a shortage of storage facilities; agro-ecological areas where farmers have plenty of experience in fruit cultivation; sizable demand for processed processed and vegetables, over 90 percent of which is met through imports and local artisanal output; and last but not least, the existence of training institutions specializing in food industries. 
Table 5.1: Sectoral breakdown of GDP

(Real growth, \%)

\begin{tabular}{|c|c|c|c|c|c|c|c|c|c|c|c|c|c|c|c|c|}
\hline & \multicolumn{4}{|c|}{ Estimates } & \multicolumn{11}{|c|}{ Projections } & \multirow[b]{2}{*}{2015} \\
\hline & 2000 & 2001 & 2002 & 2003 & 2004 & 2005 & 2006 & 2007 & 2008 & 2009 & 2010 & 2011 & 2012 & 2013 & 2014 & \\
\hline GDP at market prices & 4.8 & 4.8 & 4.2 & 4.5 & 4.9 & 5.4 & 5.8 & 6.0 & 6.3 & 6.6 & 6.8 & 7.0 & 7.0 & 7.0 & 7.0 & 7.1 \\
\hline Including: non-oil GDP & 5.1 & 5.1 & 4.6 & 4.8 & 5.3 & 5.7 & 6.0 & 6.3 & 6.5 & 6.8 & 7.0 & 7.1 & 7.1 & 7.1 & 7.1 & 7.2 \\
\hline Primary sector & 7.2 & 4.3 & 2.2 & 3.0 & 3.4 & 3.6 & 3.8 & 3.9 & 4.0 & 4.0 & 4.0 & 4.0 & 4.1 & 4.1 & 4.4 & 4.6 \\
\hline Food crop agriculture & 5.5 & 5.1 & 3.9 & 3.4 & 3.6 & 3.8 & 4.1 & 4.1 & 4.2 & 4.2 & 4.2 & 4.2 & 4.5 & 4.6 & 5.1 & 5.2 \\
\hline Industrial \& export agriculture & 9.5 & 4.8 & 2.0 & 2.8 & 2.9 & 2.7 & 2.8 & 2.9 & 2.9 & 3.1 & 3.2 & 3.3 & 3.4 & 3.5 & 4.6 & 5.4 \\
\hline Stockbreeding, gamekeeping & 9.5 & 6.9 & 4.1 & 3.3 & 3.4 & 3.8 & 4.0 & 4.0 & 4.1 & 3.9 & 3.9 & 3.8 & 4.1 & 4.2 & 3.7 & 3.1 \\
\hline Fisheries & -0.2 & 0.1 & 1.4 & 2.0 & 2.0 & 2.0 & 2.0 & 2.0 & 2.5 & 2.5 & 2.5 & 2.5 & 3.0 & 3.0 & 3.0 & 3.0 \\
\hline Silviculture and forestry & 7.0 & -0.9 & -4.2 & 2.2 & 4.0 & 4.0 & 4.5 & 5.0 & 5.0 & 5.0 & 5.0 & 5.0 & 4.0 & 3.5 & 3.0 & 3.0 \\
\hline Secondary sector & 6.6 & 5.0 & 2.9 & 4.0 & 4.0 & 4.7 & 5.7 & 6.6 & 7.6 & 8.1 & 8.5 & 8.8 & 8.8 & 8.9 & 9.0 & 9.9 \\
\hline Extractive industry & -2.1 & -2.3 & -6.4 & -2.9 & -7.5 & -5.0 & -5.0 & -5.0 & -5.0 & -5.0 & -5.0 & -5.0 & -5.0 & -5.0 & -5.0 & -5.0 \\
\hline Manufacturing industries & 6.8 & 5.6 & 3.7 & 4.6 & 5.6 & 5.8 & 7.0 & 8.1 & 9.3 & 10.0 & 10.3 & 10.6 & 10.5 & 10.5 & 10.5 & 11.7 \\
\hline Electricity, gas and water & 3.5 & 0.4 & -4.6 & 6.2 & 4.0 & 5.0 & 6.0 & 7.0 & 7.5 & 7.5 & 7.5 & 7.5 & 7.0 & 7.0 & 7.0 & 7.0 \\
\hline $\begin{array}{l}\text { CONSTRUCTION \& } \\
\text { PUBLIC WORKS }\end{array}$ & 19.6 & 14.0 & 13.0 & 7.0 & 7.2 & 7.2 & 7.5 & 7.6 & 7.7 & 7.7 & 7.6 & 7.6 & 7.6 & 7.5 & 7.5 & 7.3 \\
\hline Tertiary sector & 1.6 & 5.3 & 6.7 & 6.0 & 6.5 & 7.1 & 7.3 & 7.2 & 7.3 & 7.6 & 7.7 & 7.9 & 7.7 & 7.7 & 7.4 & 6.9 \\
\hline GDP at factor cost & 4.7 & 4.8 & 4.2 & 4.5 & 4.9 & 5.4 & 5.8 & 6.0 & 6.3 & 6.6 & 6.8 & 7.0 & 7.0 & 7.0 & 7.0 & 7.1 \\
\hline $\begin{array}{l}\text { Taxes and duties less } \\
\text { subsidies }\end{array}$ & 6.0 & 5.0 & 4.2 & 4.5 & 4.9 & 5.4 & 5.8 & 6.0 & 6.3 & 6.6 & 6.8 & 7.0 & 7.0 & 7.0 & 7.0 & 7.1 \\
\hline
\end{tabular}

Sources: IMF (1995/1996-2001)

MINFI/DP (Estimates 2002, Projections 2003-15) 
Table 5.2: Sectoral breakdown of GDP

(In billions of 1989/1990 CFAF)

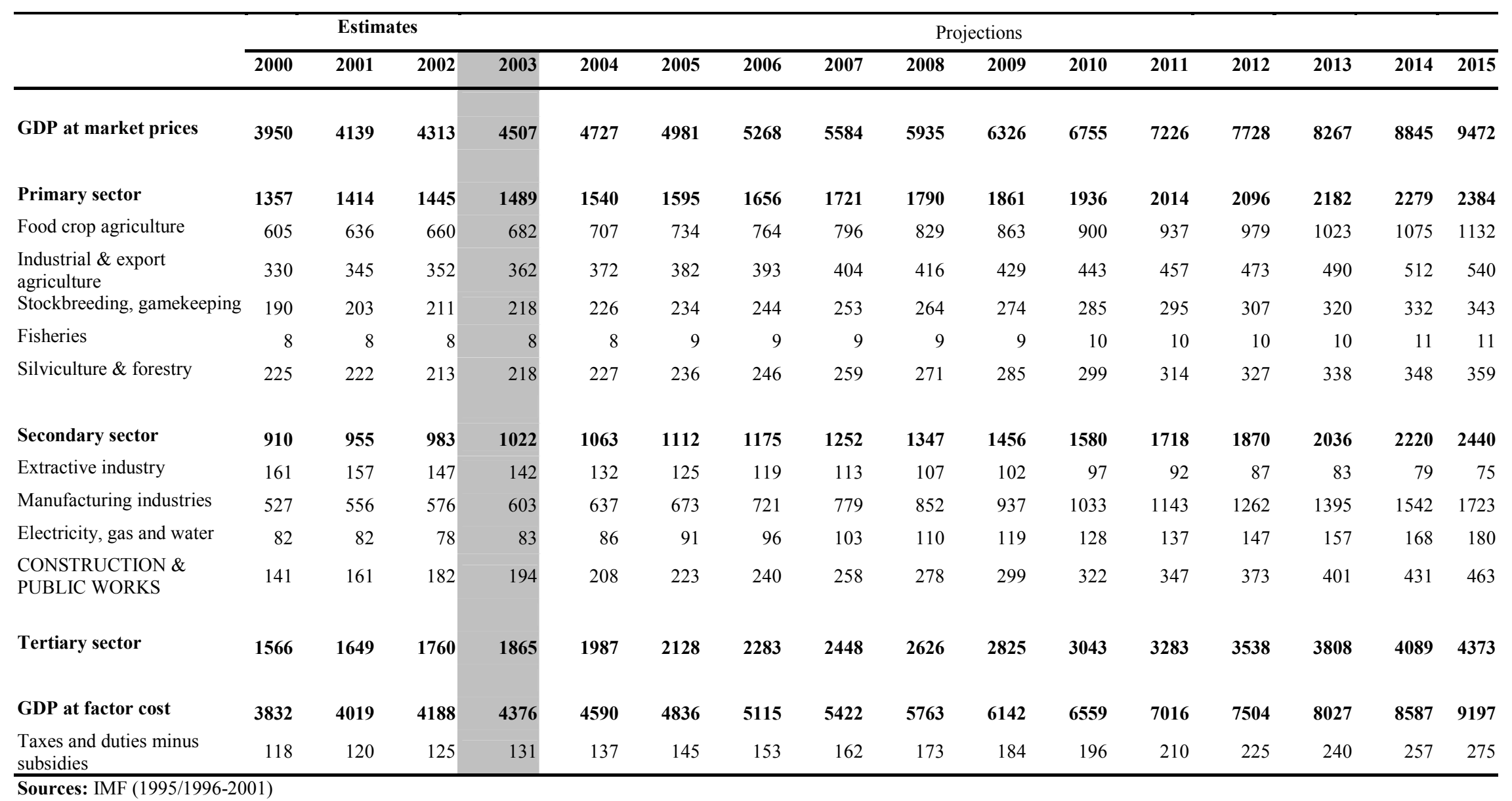

MINFI/DP (Estimates 2002, Projections 2003-15) 
Annex

Table 5.3: Sectoral breakdown of GDP

(In billions of CFAF )

\begin{tabular}{|c|c|c|c|c|c|c|c|c|c|c|c|c|c|c|c|c|}
\hline & \multicolumn{4}{|c|}{ Estimates } & \multicolumn{11}{|c|}{ Projections } & \multirow[b]{2}{*}{2015} \\
\hline & 2000 & 2001 & 2002 & 2003 & 2004 & 2005 & 2006 & 2007 & 2008 & 2009 & 2010 & 2011 & 2012 & 2013 & 2014 & \\
\hline GDP at market prices & 6074 & 6474 & 6869 & 7386 & 7887 & 8406 & 9008 & 9729 & 10544 & 11468 & 12497 & 13642 & 14893 & 16259 & 17752 & 19403 \\
\hline Primary sector & 2591 & 2743 & 2947 & 3130 & 3338 & 3562 & 3808 & 4073 & 4356 & 4660 & 4987 & 5338 & 5721 & 6140 & 6617 & 7143 \\
\hline Food crop agriculture & 1246 & 1346 & 1468 & 1559 & 1659 & 1768 & 1889 & 2019 & 2158 & 2306 & 2466 & 2636 & 2826 & 3032 & 3270 & 3531 \\
\hline $\begin{array}{l}\text { Industrial \& export } \\
\text { agriculture }\end{array}$ & 504 & 504 & 546 & 587 & 624 & 662 & 704 & 747 & 798 & 853 & 913 & 979 & 1050 & 1127 & 1223 & 1337 \\
\hline Stockbreeding, gamekeeping & 273 & 397 & 474 & 498 & 510 & 523 & 537 & 550 & 567 & 583 & 597 & 610 & 631 & 649 & 670 & 689 \\
\hline Fisheries & 12 & 13 & 14 & 14 & 15 & 16 & 16 & 17 & 18 & 19 & 20 & 21 & 22 & 23 & 25 & 26 \\
\hline Silviculture $\&$ forestry & 555 & 482 & 446 & 472 & 531 & 593 & 662 & 739 & 815 & 899 & 991 & 1092 & 1193 & 1308 & 1429 & 1560 \\
\hline Secondary sector & 1193 & 1148 & 1159 & 1212 & 1189 & 1225 & 1287 & 1375 & 1483 & 1612 & 1760 & 1931 & 2122 & 2340 & 2585 & 2883 \\
\hline Extractive industry & 237 & 190 & 171 & 175 & 133 & 118 & 108 & 102 & 97 & 92 & 88 & 83 & 79 & 75 & 71 & 68 \\
\hline Manufacturing industries & 643 & 599 & 589 & 601 & 579 & 581 & 596 & 624 & 664 & 715 & 777 & 850 & 933 & 1031 & 1143 & 1294 \\
\hline Electricity, gas and water & 91 & 93 & 93 & 104 & 114 & 125 & 139 & 157 & 177 & 200 & 225 & 254 & 286 & 321 & 361 & 405 \\
\hline $\begin{array}{l}\text { CONSTRUCTION \& } \\
\text { PUBLIC WORKS }\end{array}$ & 222 & 266 & 305 & 333 & 363 & 401 & 444 & 492 & 545 & 605 & 671 & 743 & 824 & 913 & 1010 & 1117 \\
\hline Tertiary sector & 2119 & 2400 & 2569 & 2835 & 3137 & 3382 & 3659 & 4007 & 4407 & 4873 & 5397 & 5989 & 6630 & 7321 & 8049 & 8829 \\
\hline GDP at factor cost & 5903 & 6291 & 6675 & 7178 & 7665 & 8169 & 8754 & 9454 & 10247 & 11145 & 12144 & 13257 & 14473 & 15800 & 17251 & 18855 \\
\hline $\begin{array}{l}\text { Taxes and duties minus } \\
\text { subsidies }\end{array}$ & 171 & 183 & 194 & 208 & 223 & 237 & 254 & 275 & 298 & 324 & 353 & 385 & 420 & 459 & 501 & 548 \\
\hline
\end{tabular}

Sources: IMF (1995/1996-2001)

MINFI/DP (Estimates 2002, Projections 2003-15) 
Table 5.4: Uses of GDP

(In billions of 1989/1990 CFAF)

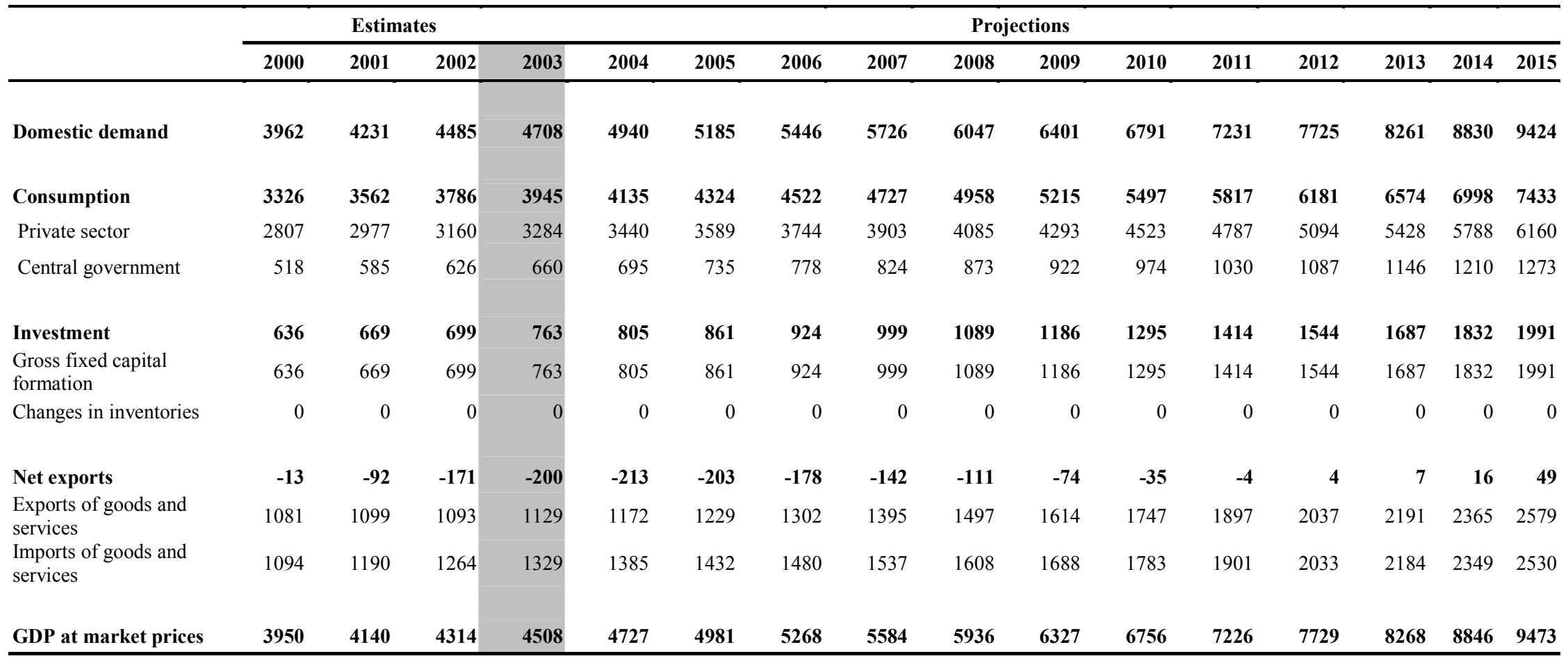

Sources: IMF (1995/1996-2001)

MINFI/DP (Estimates 2002, Projections 2003-15) 
Annex

Table 5.5: Uses of GDP

(In billions of CFAF )

\begin{tabular}{|c|c|c|c|c|c|c|c|c|c|c|c|c|c|c|c|c|}
\hline & \multicolumn{4}{|c|}{ Estimates } & \multicolumn{10}{|c|}{ Projections } & \multirow[b]{2}{*}{2014} & \multirow[b]{2}{*}{2015} \\
\hline & 2000 & 2001 & 2002 & 2003 & 2004 & 2005 & 2006 & 2007 & 2008 & 2009 & 2010 & 2011 & 2012 & 2013 & & \\
\hline Domestic demand & $\mathbf{5 8 7 7}$ & 6416 & 6972 & 7490 & 8055 & 8630 & 9247 & 9922 & 10695 & 11558 & 12520 & 13612 & 14850 & 16217 & 17701 & 19297 \\
\hline Consumption & 4835 & 5245 & 5730 & 6136 & 6598 & 7042 & 7509 & 8003 & 8559 & 9182 & 9871 & 10655 & 11551 & 12534 & 13613 & 14754 \\
\hline Private sector & 4185 & 4511 & 4922 & 5260 & 5653 & 6021 & 6407 & 6812 & 7273 & 7795 & 8377 & 9044 & 9817 & 10669 & 11603 & 12598 \\
\hline Central government & 650 & 735 & 808 & 876 & 945 & 1021 & 1102 & 1191 & 1287 & 1387 & 1494 & 1611 & 1734 & 1865 & 2009 & 2156 \\
\hline Investment & 1042 & 1171 & 1242 & 1355 & 1457 & 1588 & 1738 & 1919 & 2136 & 2376 & 2650 & 2957 & 3300 & 3683 & 4089 & 4543 \\
\hline $\begin{array}{l}\text { Gross fixed capital } \\
\text { formation }\end{array}$ & 1042 & 1171 & 1242 & 1355 & 1457 & 1588 & 1738 & 1919 & 2136 & 2376 & 2650 & 2957 & 3300 & 3683 & 4089 & 4543 \\
\hline Changes in inventories & 0 & 0 & 0 & 0 & 0 & 0 & 0 & 0 & 0 & 0 & 0 & 0 & 0 & 0 & 0 & 0 \\
\hline Net exports & 197 & 58 & -103 & -104 & -168 & -224 & -239 & -193 & -151 & -89 & -23 & 30 & 43 & 42 & 51 & 106 \\
\hline $\begin{array}{l}\text { Exports of goods and } \\
\text { services }\end{array}$ & 1901 & 1909 & 1926 & 2033 & 2060 & 2114 & 2216 & 2407 & 2625 & 2884 & 3180 & 3515 & 3846 & 4210 & 4625 & 5133 \\
\hline $\begin{array}{l}\text { Imports of goods and } \\
\text { services }\end{array}$ & 1704 & 1850 & 2029 & 2138 & 2228 & 2338 & 2455 & 2601 & 2776 & 2973 & 3203 & 3485 & 3803 & 4168 & 4574 & 5027 \\
\hline GDP at market prices & 6074 & 6474 & 6869 & 7386 & 7887 & 8406 & 9008 & 9729 & 10544 & 11468 & 12497 & 13642 & 14893 & 16259 & 17752 & 19403 \\
\hline
\end{tabular}

Sources: IMF (1995/1996-2001)

MINFI/DP (Estimates 2002, Projections 2003-15) 
Annex

Table 5.6: Deflators of Uses of GDP

\begin{tabular}{|c|c|c|c|c|c|c|c|c|c|c|c|c|c|c|c|c|}
\hline & \multicolumn{4}{|c|}{ Estimates } & \multicolumn{10}{|c|}{ Projections } & \multirow[b]{2}{*}{2014} & \multirow[b]{2}{*}{2015} \\
\hline & 2000 & 2001 & 2002 & 2003 & 2004 & 2005 & 2006 & 2007 & 2008 & 2009 & 2010 & 2011 & 2012 & 2013 & & \\
\hline Domestic demand & -2.2 & 2.2 & 2.5 & 2.3 & 2.5 & 2.1 & 2.0 & 2.1 & 2.1 & 2.1 & 2.1 & 2.1 & 2.1 & 2.1 & 2.1 & 2.1 \\
\hline Consumption & -1.0 & 1.3 & 2.8 & 2.8 & 2.6 & 2.1 & 2.0 & 2.0 & 2.0 & 2.0 & 2.0 & 2.0 & 2.0 & 2.0 & 2.0 & 2.0 \\
\hline Private sector & -1.4 & 1.6 & 2.8 & 2.8 & 2.6 & 2.1 & 2.0 & 2.0 & 2.0 & 2.0 & 2.0 & 2.0 & 2.0 & 2.0 & 2.0 & 2.0 \\
\hline Central government & 2.0 & 0.1 & 2.8 & 2.8 & 2.6 & 2.1 & 2.0 & 2.0 & 2.0 & 2.0 & 2.0 & 2.0 & 2.0 & 2.0 & 2.0 & 2.0 \\
\hline Investment & -8.1 & 6.8 & 1.6 & -0.1 & 1.9 & 1.9 & 1.9 & 2.1 & 2.1 & 2.2 & 2.2 & 2.2 & 2.2 & 2.2 & 2.2 & 2.2 \\
\hline $\begin{array}{l}\text { Gross fixed capital } \\
\text { formation } \\
\text { Changes in inventories }\end{array}$ & -8.1 & 6.8 & 1.6 & -0.1 & 1.9 & 1.9 & 1.9 & 2.1 & 2.1 & 2.2 & 2.2 & 2.2 & 2.2 & 2.2 & 2.2 & 2.2 \\
\hline Net exports & & & & & & & & & & & & & & & & \\
\hline $\begin{array}{l}\text { Exports of goods and } \\
\text { services }\end{array}$ & 24.3 & 2.7 & 1.4 & 2.2 & -2.4 & -2.1 & -1.1 & 1.3 & 1.7 & 1.9 & 1.9 & 1.9 & 1.9 & 1.8 & 1.8 & 1.8 \\
\hline $\begin{array}{l}\text { Imports of goods and } \\
\text { services }\end{array}$ & 3.4 & 2.7 & 3.2 & 0.2 & 0.1 & 1.5 & 1.7 & 2.0 & 2.0 & 2.0 & 2.0 & 2.0 & 2.0 & 2.0 & 2.0 & 2.0 \\
\hline GDP at market prices & 3.2 & 1.7 & 1.8 & 2.9 & 1.8 & 1.2 & 1.3 & 1.9 & 2.0 & 2.0 & 2.0 & 2.1 & 2.1 & 2.1 & 2.1 & 2.1 \\
\hline
\end{tabular}

Sources: IMF (1995/1996-2001)

MINFI/DP (Estimates 2002, Projections 2003-15) 
Annex

\section{Table 5.7: Uses of GDP}

(As a percentage of GDP )

\begin{tabular}{|c|c|c|c|c|c|c|c|c|c|c|c|c|c|c|c|c|}
\hline & \multicolumn{4}{|c|}{ Estimates } & \multicolumn{10}{|c|}{ Projections } & \multirow[b]{2}{*}{2014} & \multirow[b]{2}{*}{2015} \\
\hline & 2000 & 2001 & 2002 & 2003 & 2004 & 2005 & 2006 & 2007 & 2008 & 2009 & 2010 & 2011 & 2012 & 2013 & & \\
\hline Domestic demand & 96.8 & 99.1 & 101.5 & 101.4 & 102.1 & 102.7 & 102.7 & 102.0 & 101.4 & 100.8 & 100.2 & 99.8 & 99.7 & 99.7 & 99.7 & 99.5 \\
\hline Consumption & 79.6 & 81.0 & 83.4 & 83.1 & 83.7 & 83.8 & 83.4 & 82.3 & 81.2 & 80.1 & 79.0 & 78.1 & 77.6 & 77.1 & 76.7 & 76.0 \\
\hline Private sector & 68.9 & 69.7 & 71.7 & 71.2 & 71.7 & 71.6 & 71.1 & 70.0 & 69.0 & 68.0 & 67.0 & 66.3 & 65.9 & 65.6 & 65.4 & 64.9 \\
\hline General government & 10.7 & 11.3 & 11.8 & 11.9 & 12.0 & 12.1 & 12.2 & 12.2 & 12.2 & 12.1 & 12.0 & 11.8 & 11.6 & 11.5 & 11.3 & 11.1 \\
\hline Investment & 17.1 & 18.1 & 18.1 & 18.3 & 18.5 & 18.9 & 19.3 & 19.7 & 20.3 & 20.7 & 21.2 & 21.7 & 22.2 & 22.7 & 23.0 & 23.4 \\
\hline $\begin{array}{l}\text { Gross fixed capital } \\
\text { formation }\end{array}$ & 17.1 & 18.1 & 18.1 & 18.3 & 18.5 & 18.9 & 19.3 & 19.7 & 20.3 & 20.7 & 21.2 & 21.7 & 22.2 & 22.7 & 23.0 & 23.4 \\
\hline Changes in inventories & 0.0 & 0.0 & 0.0 & 0.0 & 0.0 & 0.0 & 0.0 & 0.0 & 0.0 & 0.0 & 0.0 & 0.0 & 0.0 & 0.0 & 0.0 & 0.0 \\
\hline Net exports & 3.2 & 0.9 & -1.5 & -1.4 & -2.1 & -2.7 & -2.7 & -2.0 & -1.4 & -0.8 & -0.2 & 0.2 & 0.3 & 0.3 & 0.3 & 0.5 \\
\hline $\begin{array}{l}\text { Exports of goods and } \\
\text { services }\end{array}$ & 31.3 & 29.5 & 28.0 & 27.5 & 26.1 & 25.1 & 24.6 & 24.7 & 24.9 & 25.1 & 25.4 & 25.8 & 25.8 & 25.9 & 26.1 & 26.5 \\
\hline $\begin{array}{l}\text { Imports of goods and } \\
\text { services }\end{array}$ & 28.0 & 28.6 & 29.5 & 28.9 & 28.3 & 27.8 & 27.3 & 26.7 & 26.3 & 25.9 & 25.6 & 25.5 & 25.5 & 25.6 & 25.8 & 25.9 \\
\hline GDP at market prices & 100.0 & 100.0 & 100.0 & 100.0 & 100.0 & 100.0 & 100.0 & 100.0 & 100.0 & 100.0 & 100.0 & 100.0 & 100.0 & 100.0 & 100.0 & 100.0 \\
\hline
\end{tabular}

Sources: IMF (1995/1996-2001)

MINFI/DP (Estimates 2002, Projections 2003-15) 
Table 5.8: Government flow-of-funds table (TOFE): key indicators. [Tr.: Table 5.9 is in a separate file.] (As a percentage of GDP, unless otherwise indicated )

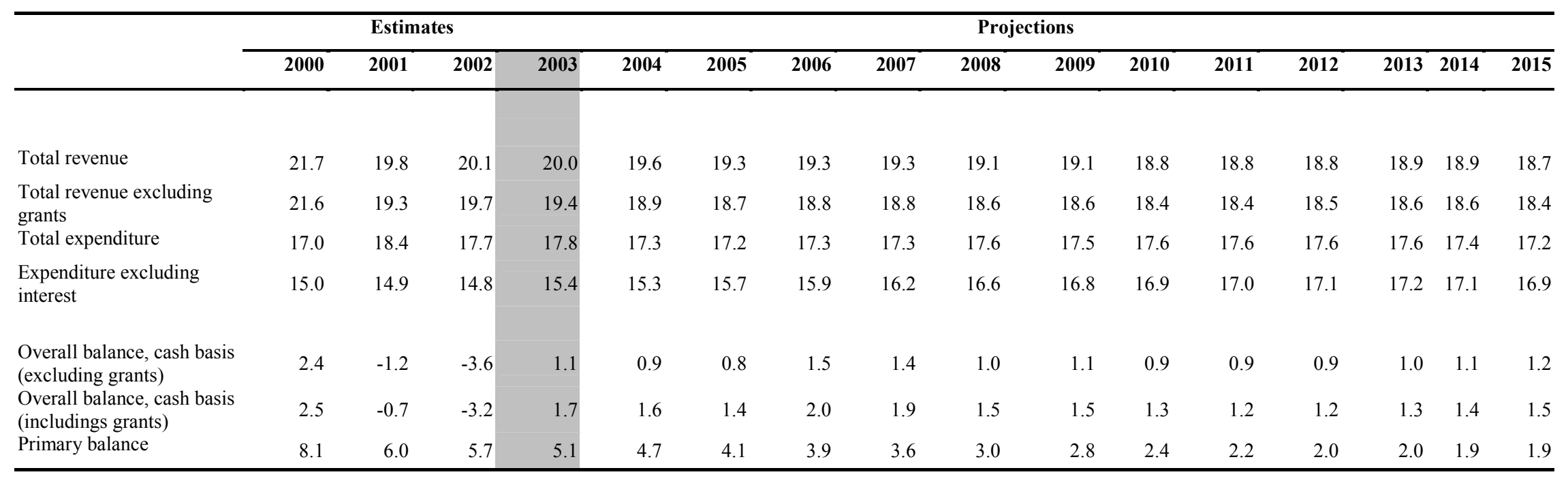

Sources: IMF (1995/1996-2001) MINFI/DP

(Estimates 2002, Projections 2003-15) 
Table 5.9.a: Government flow-of-funds table (TOFE) (Revenue and grants in billions of CFAF)_first part

\begin{tabular}{|c|c|c|c|c|c|c|c|c|c|c|c|c|c|c|c|c|}
\hline & \multicolumn{2}{|c|}{ Historical } & \multicolumn{2}{|c|}{ Estimates } & \multicolumn{12}{|c|}{ Projections } \\
\hline & 2000 & 2001 & 2002 & 2003 & 2004 & 2005 & 2006 & 2007 & 2008 & 2009 & 2010 & 2011 & 2012 & 2013 & 2014 & 2015 \\
\hline Total revenue and grants & 1320.3 & 1282.1 & 1383.1 & 1476.4 & 1544.4 & 1620. & 1739.7 & 1874.4 & 2009.8 & 2186.1 & 2352.0 & 2566.3 & 2802.2 & 3076.5 & 3346.6 & 3622.1 \\
\hline Total revenue & 1313.4 & 1251.4 & 1356.1 & 1432.4 & 1494.4 & $\begin{array}{r}1570 . \\
0\end{array}$ & 1689.7 & 1824.4 & 1959.8 & 2136.1 & 2302.0 & 2516.3 & 2752.2 & 3026.5 & 3296.6 & 3572.1 \\
\hline Oil receipts & 438.3 & 337.5 & 368.7 & 356.4 & 290.2 & 220.0 & 203.3 & 191.5 & 202.2 & 193.6 & 184.8 & 178.9 & 166.5 & 154.5 & 144.7 & 131.6 \\
\hline SNH royalties & 399.3 & 261.8 & 295.1 & 296.0 & 223.0 & 163.3 & 139.7 & 132.5 & 145.2 & 138.0 & 131.1 & 124.5 & 118.3 & 112.4 & 106.7 & 101.4 \\
\hline Chad-Cameroon pipeline revenue & 0.0 & 0.0 & 0.0 & 8.4 & 16.8 & 16.8 & 32.3 & 30.4 & 29.8 & 29.8 & 29.2 & 31.1 & 26.1 & 21.1 & 18.0 & 11.2 \\
\hline Oil taxes/companies & 39.0 & 75.7 & 73.6 & 52.0 & 50.4 & 39.9 & 31.3 & 28.6 & 27.2 & 25.8 & 24.5 & 23.3 & 22.1 & 21.0 & 20.0 & 19.0 \\
\hline Nonoil receipts & 875.1 & 913.9 & 987. & 1076.0 & 1204.2 & $\begin{array}{r}1350 . \\
0\end{array}$ & 1486.4 & 1632.9 & 1757.6 & 1942.5 & 2117.2 & 2337.5 & 2585.7 & 2872.0 & 3151.9 & 3440.6 \\
\hline Direct taxes & 184.4 & 241.0 & 276.0 & 288.0 & 323.9 & 363.2 & 393.5 & 436.9 & 471.3 & 522.6 & 580.2 & 630.6 & 702.8 & 765.9 & 834.6 & 909.8 \\
\hline Special tax on petroleum products & 55.6 & 67.2 & 66.1 & 74.0 & 83.3 & 93.2 & 104.4 & 110.7 & 117.6 & 132.3 & 141.3 & 151.2 & 161.7 & 173.0 & 185.0 & 198.2 \\
\hline Taxes on international trade & 144.3 & 162.9 & 168.2 & 190.0 & 211.1 & 235.3 & 259.4 & 275.4 & 295.0 & 317.2 & 343.2 & 375.1 & 411.5 & 453.6 & 501.0 & 554.3 \\
\hline Other taxes on goods and services & 366.9 & 388.0 & 405.1 & 453.0 & 506.2 & 572.0 & 634.6 & 703.3 & 758.7 & 846.0 & 917.6 & 1026.6 & 1118.0 & 1253.2 & 1365.7 & 1488.8 \\
\hline Incl.: VAT receipts & 147.4 & 325.8 & 341.0 & 380.0 & 423.9 & 472.1 & 511.6 & 554.1 & 597.8 & 647.0 & 701.7 & 762.6 & 830.5 & 905.1 & 986.3 & 1075.2 \\
\hline Nontax receipts & 123.8 & 54.8 & 72.0 & 71.0 & 79.7 & 86.3 & 94.4 & 106.6 & 115.0 & 124.4 & 134.9 & 154.0 & 191.7 & 226.3 & 265.5 & 289.5 \\
\hline Grants & 6.9 & 30.7 & 27.0 & 44.0 & 50.0 & 50.0 & 50.0 & 50.0 & 50.0 & 50.0 & 50.0 & 50.0 & 50.0 & 50.0 & 50.0 & 50.0 \\
\hline Total expenditure & 1029.6 & 1191.1 & 1216 & 1316.4 & 1367.8 & $\begin{array}{r}1450 . \\
0\end{array}$ & 1555.2 & 1686.9 & 1850.9 & 2012.1 & 2194.6 & 2396.8 & 2620.2 & 2858.7 & 3095.5 & 3338.7 \\
\hline Current expenditure & 800.2 & 957.9 & 1007.2 & 1054.4 & 1095.7 & $\begin{array}{r}1151 . \\
9\end{array}$ & 1227.7 & 1312.8 & 1409.6 & 1505.3 & 1610.4 & 1730.0 & 1857.8 & 1987.0 & 2143.4 & 2303.2 \\
\hline Wages & 322.5 & 350.3 & 390.1 & 425.0 & 461.6 & 505.9 & 553.4 & 606.8 & 665.9 & 727.7 & 797.1 & 869.3 & 945.3 & 1022.5 & 1116.3 & 1212.2 \\
\hline Other goods and services & 235.4 & 246.0 & 302.5 & 325.0 & 345.6 & 375.3 & 409.8 & 447.0 & 487.3 & 530.1 & 573.8 & 627.3 & 684.5 & 751.1 & 817.5 & 885.6 \\
\hline Transfers and subsidies & 104.0 & 137.0 & 115.6 & 125.0 & 131.3 & 137.8 & 142.6 & 149.7 & 157.2 & 157.2 & 157.2 & 157.2 & 157.2 & 146.2 & 146.2 & 146.2 \\
\hline Study grants and subsidies & 50.6 & 86.0 & 51.8 & 59.0 & 62.0 & 65.0 & 68.3 & 71.7 & 75.3 & 75.3 & 75.3 & 75.3 & 75.3 & 70.0 & 70.0 & 70.0 \\
\hline Pensions & 52.8 & 51.0 & 63.8 & 64.0 & 67.4 & 70.7 & 74.3 & 78.0 & 81.9 & 81.9 & 81.9 & 81.9 & 81.9 & 76.1 & 76.1 & 76.1 \\
\hline Severance (POE) & 0.6 & 0.0 & 0.0 & 2.0 & 2.0 & 2.0 & 0.0 & 0.0 & 0.0 & 0.0 & 0.0 & 0.0 & 0.0 & 0.0 & 0.0 & 0.0 \\
\hline Interest payments & 120.6 & 224.6 & 199.0 & 179.4 & 157.2 & 133.0 & 122.0 & 109.3 & 99.2 & 90.3 & 82.3 & 76.3 & 70.8 & 67.3 & 63.4 & 59.2 \\
\hline On external debt & 97.1 & 201.0 & 178.0 & 158.6 & 136.2 & 118.0 & 103.0 & 90.3 & 80.2 & 71.3 & 63.3 & 57.3 & 51.8 & 48.3 & 44.4 & 40.2 \\
\hline On domestic debt & 23.5 & 23.6 & 21.0 & 20.8 & 21.0 & 15.0 & 19.0 & 19.0 & 19.0 & 19.0 & 19.0 & 19.0 & 19.0 & 19.0 & 19.0 & 19.0 \\
\hline $\begin{array}{l}\text { Repayments of TCA/TVA } \\
\text { credits }\end{array}$ & 17.7 & 0.0 & 0.0 & 0.0 & 0.0 & 0.0 & 0.0 & 0.0 & 0.0 & 0.0 & 0.0 & 0.0 & 0.0 & 0.0 & 0.0 & 0.0 \\
\hline Capital expenditure & 219.2 & 233.2 & 192.0 & 262.0 & 272.1 & 298.1 & 327.5 & 374.1 & 441.2 & 506.8 & 584.2 & 666.8 & 762.3 & 871.7 & 952.1 & 1035.5 \\
\hline $\begin{array}{l}\text { Including: dépenses/financements } \\
\text { ext. }\end{array}$ & 47.9 & 76.0 & 30.0 & 67.0 & 80.4 & 88.4 & 97.3 & 107.0 & 107.0 & 107.0 & 107.0 & 101.6 & 91.5 & 82.3 & 74.1 & 74.1 \\
\hline Statistical adjustment & 10.2 & 20.4 & 17.0 & 0.0 & 0.0 & 0.0 & 0.0 & 0.0 & 0.0 & 0.0 & 0.0 & 0.0 & 0.0 & 0.0 & 0.0 & 0.0 \\
\hline $\begin{array}{l}\text { Overall balance, commitment basis } \\
\text { (excluding grants) }\end{array}$ & 0.0 & 60.3 & 139.9 & 115.9 & 126.6 & 120.0 & 134.4 & 137.5 & 108.9 & 124.0 & 107.4 & 119.6 & 132.0 & 167.8 & 201.1 & 233.5 \\
\hline Overall balance, commitment basis & 290.7 & 91.0 & 166.9 & 159.9 & 176.6 & 170.0 & 184.4 & 187.5 & 158.9 & 174.0 & 157.4 & 169.6 & 182.0 & 217.8 & 251.1 & 283.5 \\
\hline Changes in payment arrears & -140.4 & -136.0 & -385.0 & -35.0 & -53.9 & -53.7 & 0.0 & 0.0 & 0.0 & 0.0 & 0.0 & 0.0 & 0.0 & 0.0 & 0.0 & 0.0 \\
\hline External & -8.8 & -33.0 & -357.0 & 0.0 & 0.0 & 0.0 & 0.0 & 0.0 & 0.0 & 0.0 & 0.0 & 0.0 & 0.0 & 0.0 & 0.0 & 0.0 \\
\hline Domestic & -131.6 & -103.0 & -28.0 & -35.0 & -53.9 & -53.7 & 0.0 & 0.0 & 0.0 & 0.0 & 0.0 & 0.0 & 0.0 & 0.0 & 0.0 & 0.0 \\
\hline $\begin{array}{l}\text { Overall balance, cash basis } \\
\text { (excluding grants) }\end{array}$ & 143.4 & -75.7 & -245.1 & 80.9 & 72.7 & 66.3 & 134.4 & 137.5 & 108.9 & 124.0 & 107.4 & 119.6 & 132.0 & 167.8 & 201.1 & 233.5 \\
\hline Overall balance, cash basis & 150.3 & -45.0 & -218.1 & 124.9 & 122.7 & 116.3 & 184.4 & 187.5 & 158.9 & 174.0 & 157.4 & 169.6 & 182.0 & 217.8 & 251.1 & 283.5 \\
\hline
\end{tabular}


Table 5.9-b: Government flow-of-funds table (TOFE) (Revenue and grants in billions of CFAF)_second part

\begin{tabular}{|c|c|c|c|c|c|c|c|c|c|c|c|c|c|c|c|c|}
\hline & \multicolumn{2}{|c|}{ Historical } & \multicolumn{2}{|c|}{ Estimates } & \multicolumn{12}{|c|}{ Projections } \\
\hline & 2000 & 2001 & 2002 & 2003 & 2004 & 2005 & 2006 & 2007 & 2008 & 2009 & 2010 & 2011 & 2012 & 2013 & 2014 & 2015 \\
\hline Financing & -150.3 & 45.0 & 244.7 & -124.9 & -122.7 & -116.3 & -184.4 & -187.5 & -158.9 & -174.0 & -157.4 & -169.6 & -182.0 & -217.8 & -251.1 & -283.5 \\
\hline Net external financing & -51.0 & 125.0 & 405.3 & 80.6 & 118.1 & 115.5 & 107.3 & 64.2 & 98.6 & 73.9 & 75.6 & 7.4 & 3.7 & -17.9 & -69.2 & -89.7 \\
\hline External debt amortization & -124.9 & -332.0 & -347.0 & -319.5 & -289.2 & -271.8 & -239.4 & -201.1 & -191.5 & -174.7 & -152.0 & -138.1 & -114.3 & -112.5 & -109.7 & -114.4 \\
\hline Drawings $1 /$ & 73.9 & 84.0 & 74.3 & 126.0 & 193.3 & 187.6 & 178.6 & 135.7 & 172.2 & 150.6 & 149.0 & 76.2 & 70.0 & 56.0 & 19.5 & 24.7 \\
\hline Projects 1/ & 47.9 & 76.0 & 30.0 & 67.0 & 78.0 & 83.0 & 87.0 & 0.0 & 0.0 & 0.0 & 0.0 & 0.0 & 0.0 & 0.0 & 0.0 & 0.0 \\
\hline Programs 1/ & 26.0 & 8.0 & 44.3 & 59.0 & 40.0 & 40.0 & 40.0 & 0.0 & 0.0 & 0.0 & 0.0 & 0.0 & 0.0 & 0.0 & 0.0 & 0.0 \\
\hline $\begin{array}{l}\text { External debt relief (excl. C2D-Non- } \\
\text { Indebtedness and Development } \\
\text { Contract) }\end{array}$ & 0.0 & 396.0 & 901.0 & 274.1 & 213.9 & 199.7 & 168.0 & 129.6 & 117.8 & 97.9 & 78.7 & 69.4 & 47.9 & 38.6 & 21.0 & 0.0 \\
\hline including: HIPC & 0.0 & 0.0 & 51.0 & 37.0 & 46.0 & 72.0 & 60.0 & 56.0 & 53.0 & 49.0 & 50.0 & 51.0 & 54.0 & 47.0 & 52.0 & 56.0 \\
\hline Reduction of arrears on principal & 0.0 & -23.0 & -223.0 & 0.0 & 0.0 & 0.0 & 0.0 & 0.0 & 0.0 & 0.0 & 0.0 & 0.0 & 0.0 & 0.0 & 0.0 & 0.0 \\
\hline Net domestic financing (identified) & -99.3 & -80.0 & -160.6 & -205.5 & -240.8 & -231.8 & -291.8 & -251.7 & -257.5 & -247.9 & -233.0 & -177.0 & -185.7 & -199.9 & -181.9 & -193.8 \\
\hline Net banking system (change in PNG) & -89.6 & 25.0 & 25.0 & -41.5 & -51.8 & -25.8 & -68.8 & -82.1 & -79.2 & -75.0 & -63.5 & -63.3 & -66.3 & -71.7 & -48.6 & -49.4 \\
\hline IMF, net & 38.1 & 15.5 & 6.6 & 20.7 & 17.0 & -22.0 & -23.0 & -30.0 & -27.0 & -17.0 & -7.0 & 0.0 & 0.0 & 0.0 & 0.0 & 0.0 \\
\hline Other & -80.7 & 0.0 & 0.0 & -62.2 & -68.8 & -3.8 & -45.8 & -52.1 & -52.2 & -58.0 & -56.5 & -63.3 & -66.3 & -71.7 & -48.6 & -49.4 \\
\hline BEAC & 0.0 & 0.0 & 0.0 & -55.4 & -116.8 & 7.0 & -35.0 & -41.3 & -41.4 & -47.2 & -45.7 & -52.5 & -55.5 & -60.9 & -37.8 & -38.6 \\
\hline BCM & 0.0 & 0.0 & 0.0 & -6.8 & 48.0 & -10.8 & -10.8 & -10.8 & -10.8 & -10.8 & -10.8 & -10.8 & -10.8 & -10.8 & -10.8 & -10.8 \\
\hline $\begin{array}{l}\text { Amortization of agreed debt (dettes } \\
\text { conventionnées) }\end{array}$ & -18.3 & -58.0 & -110.0 & -108.0 & -134.0 & -137.0 & -149.0 & -90.3 & -93.5 & -82.1 & -71.8 & -8.4 & -5.6 & -5.1 & 0.0 & 0.0 \\
\hline Net nonbank financing & -19.2 & -25.0 & -15.0 & 0.0 & 0.0 & 0.0 & 0.0 & 0.0 & 0.0 & 0.0 & 0.0 & 0.0 & 0.0 & 0.0 & 0.0 & 0.0 \\
\hline $\begin{array}{l}\text { Escrow accounts, special tax on } \\
\text { petroleum products (TSPP) }\end{array}$ & 0.0 & 0.0 & 0.0 & 0.0 & 0.0 & 0.0 & 0.0 & 0.0 & 0.0 & 0.0 & 0.0 & 0.0 & 0.0 & 0.0 & 0.0 & 0.0 \\
\hline Financial sector & 0.0 & 0.0 & 0.0 & 0.0 & 0.0 & 0.0 & 0.0 & 0.0 & 0.0 & 0.0 & 0.0 & 0.0 & 0.0 & 0.0 & 0.0 & 0.0 \\
\hline Privatization & 64.6 & 23.0 & 3.0 & 4.0 & 8.0 & 0.0 & 0.0 & 0.0 & 0.0 & 0.0 & 0.0 & 0.0 & 0.0 & 0.0 & 0.0 & 0.0 \\
\hline Refund of VAT credit & -15.2 & -29.5 & -53.7 & -48.0 & -53.0 & -59.0 & -63.9 & -69.3 & -74.7 & -80.9 & -87.7 & -95.3 & -103.8 & -113.1 & -123.3 & -134.4 \\
\hline $\begin{array}{l}\text { Deposits of oil taxes/companies for } \\
\text { regularization }\end{array}$ & 0.0 & 0.0 & 0.0 & 0.0 & 0.0 & 0.0 & 0.0 & 0.0 & 0.0 & 0.0 & 0.0 & 0.0 & 0.0 & 0.0 & 0.0 & 0.0 \\
\hline $\begin{array}{l}\text { Net change in anticipated debits and } \\
\text { credits }\end{array}$ & -20.6 & -52.5 & -9.9 & -12.0 & -10.0 & -10.0 & -10.0 & -10.0 & -10.0 & -10.0 & -10.0 & -10.0 & -10.0 & -10.0 & -10.0 & -10.0 \\
\hline Impact of SDR exchange rate & 0.7 & 0.0 & 0.0 & 0.0 & 0.0 & 0.0 & 0.0 & 0.0 & 0.0 & 0.0 & 0.0 & 0.0 & 0.0 & 0.0 & 0.0 & 0.0 \\
\hline Securitization & 63.0 & 37.0 & 0.0 & 0.0 & 0.0 & 0.0 & 0.0 & 0.0 & 0.0 & 0.0 & 0.0 & 0.0 & 0.0 & 0.0 & 0.0 & 0.0 \\
\hline \multicolumn{17}{|l|}{ Memorandum items; } \\
\hline \multicolumn{4}{|l|}{ Financing requirements $1 /$} & & 193.3 & 187.6 & 178.6 & 135.7 & 172.2 & 150.6 & 149.0 & 76.2 & 70.0 & 56.0 & 19.5 & 24.7 \\
\hline \multirow{2}{*}{\multicolumn{4}{|c|}{$\begin{array}{l}\text { Requirements excluding debt amortization } \\
\text { Possible relief under the C2D 2/ }\end{array}$}} & 0.0 & 59.3 & 50.6 & 29.6 & 45.4 & 78.7 & 68.5 & 77.2 & 67.8 & 64.4 & 50.9 & 19.5 & 24.7 \\
\hline & & & & 47.0 & 57.8 & 64.0 & 64.9 & 61.6 & 56.3 & 52.8 & 48.6 & 39.8 & 32.3 & 25.8 & 24.6 & 22.7 \\
\hline
\end{tabular}

2/ C2D will represent half of the amount posted in 2004 if the completion point is reached after March 31, 2004

Sources: MINEFI/ DP, CAA 
Table 5.10: Balance of payments

(In billions of CFAF)

\begin{tabular}{|c|c|c|c|c|c|c|c|c|c|c|c|c|c|c|c|}
\hline & \multicolumn{2}{|c|}{ Estimates } & \multicolumn{13}{|c|}{ Projections } \\
\hline & 2001 & 2002 & 2003 & 2004 & 2005 & 2006 & 2007 & 2008 & 2009 & 2010 & 2011 & 2012 & 2013 & 2014 & 2015 \\
\hline Exports of goods & 1614.7 & 1360.5 & 1396.9 & 1450.6 & 1467.5 & 1579.2 & 1655.1 & 1793.8 & 1951.9 & 2096.1 & 2259.3 & 2487.6 & 62714.5 & 2966.7 & 3253.8 \\
\hline \multirow[b]{2}{*}{ Imports of goods } & - & - & - & - & - & - & - & - & - & - & - & & - & - & \\
\hline & 1300.0 & 1362.2 & 1385.8 & 1390.7 & 1449.8 & 1520.0 & 1595.7 & 1695.4 & 1816.4 & 1952.9 & 2111.4 & 2305.0 & 2523.6 & 2774.5 & 3054.8 \\
\hline Trade balance & 314.7 & -1.7 & 11.1 & 59.8 & 17.8 & 59.2 & 59.4 & 98.4 & 135.5 & 143.2 & 148.0 & 182.6 & 190.9 & 192.1 & 198.9 \\
\hline Nonfactor services (net) & -256.6 & -411.2 & -115.3 & -228.0 & -241.5 & -298.2 & -252.8 & -249.0 & -224.9 & -166.5 & -117.5 & -139.6 & $6-148.9$ & -141.2 & -92.7 \\
\hline Credit & 293.9 & 565.4 & 636.5 & 609.6 & 646.3 & 636.9 & 752.1 & 831.2 & 931.9 & 1083.4 & 1256.0 & 1358.3 & 31495.3 & 1658.7 & 1879.5 \\
\hline Debit & -550.5 & -666.5 & -751.8 & -837.7 & -887.7 & -935.1 & 1005.0 & 1080.2 & 1156.7 & 1250.0 & 1373.6 & 1497.9 & 1644.2 & 1799.9 & 1972.2 \\
\hline $\begin{array}{l}\text { Balance of goods and } \\
\text { services (nonfactor) }\end{array}$ & 58.1 & -413.0 & -104.3 & -168.2 & -223.7 & -239.0 & -193.4 & -150.6 & -89.4 & -23.4 & 30.5 & 43.1 & 42.0 & 50.9 & 106.2 \\
\hline Factor income & -252.9 & -243.6 & -151.7 & -126.4 & -106.3 & -90.1 & -76.4 & -65.4 & -55.6 & -46.7 & -39.8 & -33.4 & -28.9 & -24.0 & -18.7 \\
\hline Credit & 10.3 & 11.4 & 12.0 & 12.7 & 13.4 & - $\quad 14.2$ & 14.9 & 15.8 & 16.6 & 17.5 & 18.5 & 19.5 & 20.6 & 21.8 & 23.0 \\
\hline Debit & -263.2 & -255.0 & -163.8 & -139.1 & -119.8 & -104.2 & -91.3 & -81.2 & -72.2 & -64.3 & -58.3 & -52.9 & -49.5 & -45.7 & -41.6 \\
\hline $\begin{array}{l}\text { Interest on guaranteed } \\
\text { public external debt }\end{array}$ & -189.1 & -178.0 & -158.6 & -136.2 & -118.0 & -103.0 & -90.3 & -80.2 & -71.3 & -63.3 & -57.3 & -51.8 & -48.3 & -44.4 & -40.2 \\
\hline $\begin{array}{l}\text { Interest on private } \\
\text { external debt etc. }\end{array}$ & -74.1 & -77.0 & -5.1 & -2.9 & -1.8 & -1.3 & -1.0 & -0.9 & -0.9 & -1.0 & -1.0 & -1.1 & -1.2 & -1.3 & -1.4 \\
\hline Unrequited transfers & 114.2 & 116.6 & 48.4 & 56.1 & 57.6 & 58.9 & 60.1 & 59.3 & 58.3 & 57.1 & 55.8 & 54.4 & 52.9 & 51.4 & 49.8 \\
\hline Private & 11.5 & 14.3 & 13.6 & 15.3 & 16.8 & 18.1 & 19.3 & 18.5 & 17.5 & 16.3 & 15.0 & 13.6 & 12.1 & 10.6 & 9.0 \\
\hline Credit & 52.5 & 56.7 & 59.5 & 61.3 & 63.1 & 64.9 & 66.8 & 66.8 & 66.8 & 66.8 & 66.8 & 66.8 & 66.8 & 66.8 & 66.8 \\
\hline Debit & -41.0 & -42.4 & -45.9 & -46.0 & -46.3 & -46.8 & -47.4 & -48.3 & -49.3 & -50.5 & -51.8 & -53.2 & -54.6 & -56.2 & -57.7 \\
\hline Public net & 102.7 & 102.3 & 34.8 & 40.8 & 40.8 & 40.8 & 40.8 & 40.8 & 40.8 & 40.8 & 40.8 & 40.8 & 40.8 & 40.8 & 40.8 \\
\hline $\begin{array}{l}\text { Current account } \\
\text { balance: inclusive of } \\
\text { public transfers }\end{array}$ & -80.6 & -539.9 & -207.5 & -238.5 & -272.4 & -270.1 & -209.6 & -156.7 & -86.7 & -13.0 & 46.5 & 64.0 & 66.1 & 78.4 & 137.4 \\
\hline $\begin{array}{l}\text { Current account } \\
\text { balance: exclusive of } \\
\text { public transfers }\end{array}$ & -183.3 & -642.2 & -242.3 & -279.3 & -313.2 & -310.9 & -250.4 & -197.5 & -127.5 & -53.8 & 5.7 & 23.2 & 25.3 & 37.6 & 96.6 \\
\hline Capital movements & 235.2 & 355.9 & 102.5 & 167.1 & 177.9 & 191.8 & 155.8 & 196.9 & 179.2 & 188.3 & 128.1 & 175.4 & 165.8 & 127.3 & 120.8 \\
\hline I. LONG TERM & 213.2 & 355.5 & 102.2 & 166.7 & 177.5 & 191.4 & 155.4 & 196.5 & 178.8 & 187.9 & 127.7 & 175.0 & 165.5 & 127.0 & 120.5 \\
\hline A. Public & -174.7 & 378.7 & 80.6 & 118.1 & 115.5 & 107.3 & 64.2 & 98.6 & 73.9 & 75.6 & 7.4 & 3.7 & -17.9 & -69.2 & -89.7 \\
\hline Drawings & 106.5 & 74.3 & 126.0 & 193.3 & 187.6 & 178.6 & 135.7 & 172.2 & 150.6 & 149.0 & 76.2 & 70.0 & 56.0 & 19.5 & 24.7 \\
\hline Amortizations owed & -281.2 & -347.0 & -319.5 & -289.2 & -271.8 & -239.4 & -201.1 & -191.5 & -174.7 & -152.0 & -138.1 & -114.3 & -112.5 & -109.7 & -114.4 \\
\hline External debt relief & 0.0 & 901.0 & 274.1 & 213.9 & 199.7 & 168.0 & 129.6 & 117.8 & 97.9 & 78.7 & 69.4 & 47.9 & 38.6 & 21.0 & 0.0 \\
\hline Including: HIPC & 0.0 & 51.0 & 37.0 & 46.0 & 72.0 & 60.0 & 56.0 & 53.0 & 49.0 & 50.0 & 51.0 & 54.0 & 47.0 & 52.0 & 56.0 \\
\hline $\begin{array}{l}\text { Reduction of arrears } \\
\text { on principal }\end{array}$ & 0.0 & -223.0 & 0.0 & 0.0 & 0.0 & 0.0 & 0.0 & 0.0 & 0.0 & 0.0 & 0.0 & 0.0 & 0.0 & 0.0 & 0.0 \\
\hline B. Private & 387.9 & -23.2 & 21.6 & 48.7 & 62.0 & 84.1 & 91.2 & 97.9 & 104.9 & 112.3 & 120.3 & 171.3 & 183.3 & 196.2 & 210.1 \\
\hline $\begin{array}{l}\text { Foreign Direct } \\
\text { Investment }\end{array}$ & 0.0 & 33.2 & 49.6 & 62.5 & 68.6 & 87.0 & 92.2 & 98.0 & 104.5 & 111.6 & 119.3 & 170.2 & 182.1 & 194.8 & 208.6 \\
\hline Prive & 387.9 & -56.4 & -28.0 & -13.8 & -6.6 & -2.9 & -1.0 & -0.1 & 0.4 & 0.7 & 1.0 & 1.1 & 1.3 & 1.4 & 1.6 \\
\hline II. SH & 22.0 & 0.4 & 0.4 & 0.4 & 0.4 & 0.4 & 0.4 & 0.4 & 0.4 & 0.4 & 0.4 & 0.4 & 0.4 & 0.4 & 0.4 \\
\hline Errors and omissions & -64.2 & 0.0 & 0.0 & 0.0 & 0.0 & 0.0 & 0.0 & 0.0 & 0.0 & 0.0 & 0.0 & 0.0 & 0.0 & 0.0 & 0.0 \\
\hline Overall balance & 90.4 & -184.1 & -105.0 & -71.3 & -94.6 & -78.3 & -53.9 & 40.2 & 92.5 & 175.3 & 174.5 & 239.4 & 231.9 & 205.7 & 258.3 \\
\hline Financing & -90.4 & 184.1 & 105.0 & 71.3 & 94.6 & 78.3 & 53.9 & -40.2 & -92.5 & -175.3 & -174.5 & -239.4 & -231.9 & -205.7 & -258.3 \\
\hline
\end{tabular}


Table 5.11: Proposed apportionment of budget appropriations by sector (in billions of CFAF)

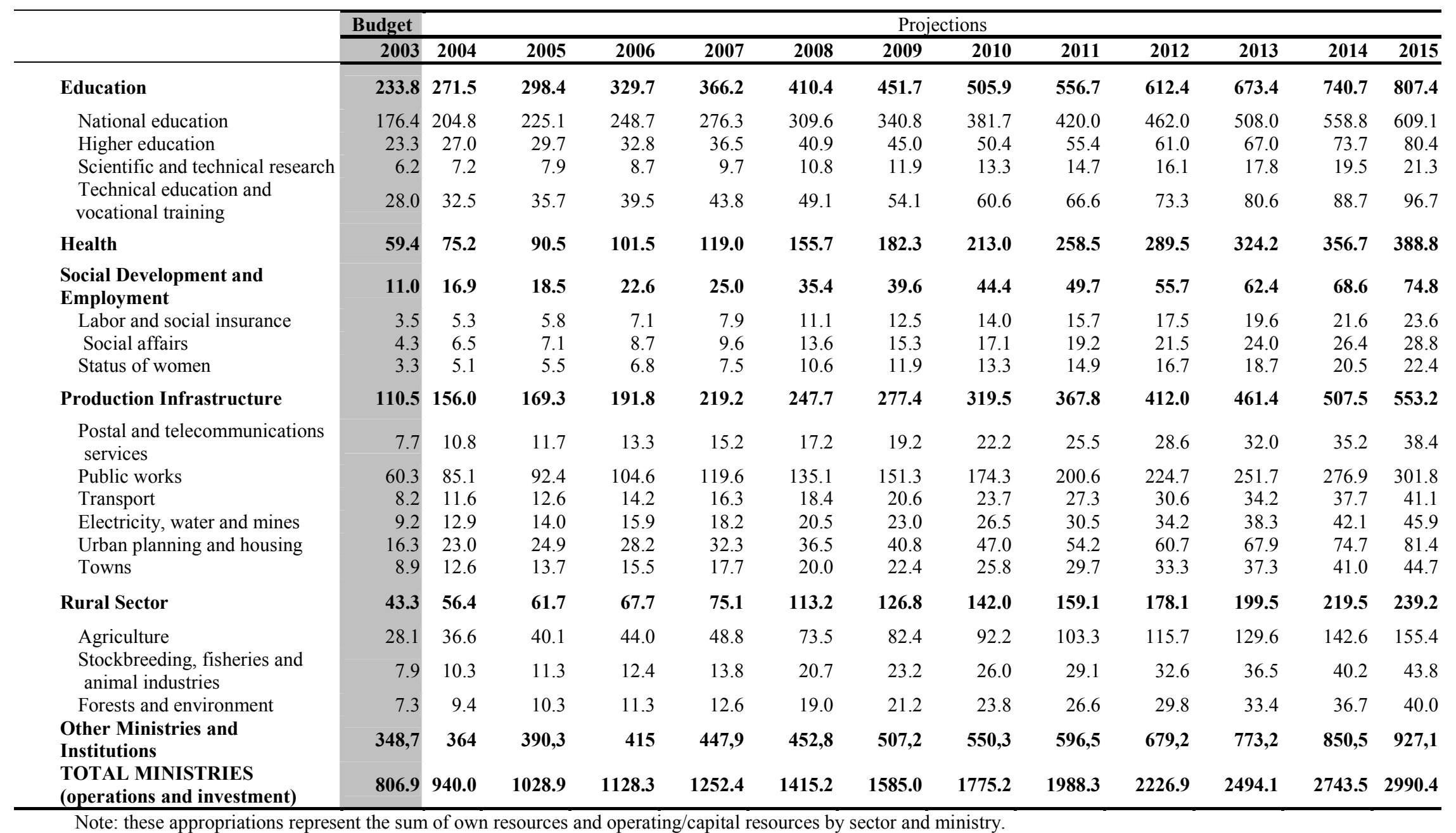

Note: these appropriations represent the sum of own resources and operating/capital resources by sector and ministry.

Source: $\mathrm{MINFI} / \mathrm{DP}$ 
Table 5.12. MEDIUM-TERM EXPENDITURE FRAMEWORK: classification budget (limited to operating expenditures and capital expenditures funded domestically)

\begin{tabular}{|c|c|c|c|c|c|c|c|c|c|c|c|c|c|}
\hline & 2003 & 2004 & 2005 & 2006 & 2007 & 2008 & 2009 & 2010 & 2011 & 2012 & 2013 & 2014 & 2015 \\
\hline \multicolumn{14}{|c|}{ I. As a percentage of GDP. } \\
\hline Education & 3.2 & 3.4 & 3.6 & 3.7 & 3.8 & 3.9 & 3.9 & 4.0 & 4.1 & 4.1 & 4.1 & 4.2 & 4.2 \\
\hline National education & 2.4 & 2.6 & 2.7 & 2.8 & 2.8 & 2.9 & 3.0 & 3.1 & 3.1 & 3.1 & 3.1 & 3.1 & 3.1 \\
\hline Higher education & 0.3 & 0.3 & 0.4 & 0.4 & 0.4 & 0.4 & 0.4 & 0.4 & 0.4 & 0.4 & 0.4 & 0.4 & 0.4 \\
\hline Scientific and technical research & 0.1 & 0.1 & 0.1 & 0.1 & 0.1 & 0.1 & 0.1 & 0.1 & 0.1 & 0.1 & 0.1 & 0.1 & 0.1 \\
\hline $\begin{array}{l}\text { Technical education and vocational } \\
\text { training }\end{array}$ & 0.4 & 0.4 & 0.4 & 0.4 & 0.5 & 0.5 & 0.5 & 0.5 & 0.5 & 0.5 & 0.5 & 0.5 & 0.5 \\
\hline Health & 0.8 & 1.0 & 1.1 & 1.1 & 1.2 & 1.5 & 1.6 & 1.7 & 1.9 & 1.9 & 2.0 & 2.0 & 2.0 \\
\hline Social Development and Employment & 0.1 & 0.2 & 0.2 & 0.3 & 0.3 & 0.3 & 0.3 & 0.4 & 0.4 & 0.4 & 0.4 & 0.4 & 0.4 \\
\hline Labor and social insurance & 0.0 & 0.1 & 0.1 & 0.1 & 0.1 & 0.1 & 0.1 & 0.1 & 0.1 & 0.1 & 0.1 & 0.1 & 0.1 \\
\hline Social affairs & 0.1 & 0.1 & 0.1 & 0.1 & 0.1 & 0.1 & 0.1 & 0.1 & 0.1 & 0.1 & 0.1 & 0.1 & 0.1 \\
\hline Status of women & 0.0 & 0.1 & 0.1 & 0.1 & 0.1 & 0.1 & 0.1 & 0.1 & 0.1 & 0.1 & 0.1 & 0.1 & 0.1 \\
\hline Production infrastructure & 1.5 & 2.0 & 2.0 & 2.1 & 2.3 & 2.3 & 2.4 & 2.6 & 2.7 & 2.8 & 2.8 & 2.9 & 2.9 \\
\hline Postal and telecommunications services & 0.1 & 0.1 & 0.1 & 0.1 & 0.2 & 0.2 & 0.2 & 0.2 & 0.2 & 0.2 & 0.2 & 0.2 & 0.2 \\
\hline Public works & 0.8 & 1.1 & 1.1 & 1.2 & 1.2 & 1.3 & 1.3 & 1.4 & 1.5 & 1.5 & 1.5 & 1.6 & 1.6 \\
\hline Transport & 0.1 & 0.1 & 0.1 & 0.2 & 0.2 & 0.2 & 0.2 & 0.2 & 0.2 & 0.2 & 0.2 & 0.2 & 0.2 \\
\hline Electricity, water and mines & 0.1 & 0.2 & 0.2 & 0.2 & 0.2 & 0.2 & 0.2 & 0.2 & 0.2 & 0.2 & 0.2 & 0.2 & 0.2 \\
\hline Urban planning & 0.2 & 0.3 & 0.3 & 0.3 & 0.3 & 0.3 & 0.4 & 0.4 & 0.4 & 0.4 & 0.4 & 0.4 & 0.4 \\
\hline Towns & 0.1 & 0.2 & 0.2 & 0.2 & 0.2 & 0.2 & 0.2 & 0.2 & 0.2 & 0.2 & 0.2 & 0.2 & 0.2 \\
\hline Rural Sector & 0.6 & 0.7 & 0.7 & 0.8 & 0.8 & 1.1 & 1.1 & 1.1 & 1.2 & 1.2 & 1.2 & 1.2 & 1.2 \\
\hline Agriculture & 0.4 & 0.5 & 0.5 & 0.5 & 0.5 & 0.7 & 0.7 & 0.7 & 0.8 & 0.8 & 0.8 & 0.8 & 0.8 \\
\hline $\begin{array}{l}\text { Stockbreeding, fisheries and animal } \\
\text { industries }\end{array}$ & 0.1 & 0.1 & 0.1 & 0.1 & 0.1 & 0.2 & 0.2 & 0.2 & 0.2 & 0.2 & 0.2 & 0.2 & 0.2 \\
\hline Forests and environment & 0.1 & 0.1 & 0.1 & 0.1 & 0.1 & 0.2 & 0.2 & 0.2 & 0.2 & 0.2 & 0.2 & 0.2 & 0.2 \\
\hline Other Ministries and Institutions & 4.4 & 4.3 & 4.5 & 4.5 & 4.4 & 4.3 & 4.1 & 4.3 & 4.2 & 4.2 & 4.3 & 4.5 & 4.5 \\
\hline Total Ministerial Budget & 10.9 & 11.9 & 12.2 & 12.5 & 12.9 & 13.4 & 13.8 & 14.2 & 14.6 & 15.0 & 15.3 & 15.5 & 15.4 \\
\hline \multicolumn{14}{|c|}{ II. As a Percentage of Total Expenditure } \\
\hline Education & 15.5 & 17.6 & 18.7 & 19.5 & 20.2 & 20.9 & 21.4 & 22.1 & 22.4 & 22.7 & 23.0 & 23.4 & 23.7 \\
\hline National education & 11.7 & 13.3 & 14.1 & 14.7 & 15.3 & 15.8 & 16.1 & 16.7 & 16.9 & 17.1 & 17.3 & 17.7 & 17.9 \\
\hline Higher education & 1.5 & 1.8 & 1.9 & 1.9 & 2.0 & 2.1 & 2.1 & 2.2 & 2.2 & 2.3 & 2.3 & 2.3 & 2.4 \\
\hline Scientific and technical research & 0.4 & 0.5 & 0.5 & 0.5 & 0.5 & 0.6 & 0.6 & 0.6 & 0.6 & 0.6 & 0.6 & 0.6 & 0.6 \\
\hline $\begin{array}{l}\text { Technical education and vocational } \\
\text { training }\end{array}$ & 1.9 & 2.1 & 2.2 & 2.3 & 2.4 & 2.5 & 2.6 & 2.7 & 2.7 & 2.7 & 2.8 & 2.8 & 2.8 \\
\hline Health & 3.9 & 4.9 & 5.7 & 6.0 & 6.6 & 7.9 & 8.6 & 9.3 & 10.4 & 10.7 & 11.1 & 11.3 & 11.4 \\
\hline Social Development and Employment & 0.7 & 1.1 & 1.2 & 1.3 & 1.4 & 1.8 & 1.9 & 1.9 & 2.0 & 2.1 & 2.1 & 2.2 & 2.2 \\
\hline Labor and social insurance & 0.2 & 0.3 & 0.4 & 0.4 & 0.4 & 0.6 & 0.6 & 0.6 & 0.6 & 0.7 & 0.7 & 0.7 & 0.7 \\
\hline Social affairs & 0.3 & 0.4 & 0.4 & 0.5 & 0.5 & 0.7 & 0.7 & 0.7 & 0.8 & 0.8 & 0.8 & 0.8 & 0.8 \\
\hline Status of women & 0.2 & 0.3 & 0.3 & 0.4 & 0.4 & 0.5 & 0.6 & 0.6 & 0.6 & 0.6 & 0.6 & 0.6 & 0.7 \\
\hline Production infrastructure & 7.3 & 10.1 & 10.6 & 11.3 & 12.1 & 12.6 & 13.1 & 14.0 & 14.8 & 15.3 & 15.7 & 16.0 & 16.3 \\
\hline Postal and telecommunications services & 0.5 & 0.7 & 0.7 & 0.8 & 0.8 & 0.9 & 0.9 & 1.0 & 1.0 & 1.1 & 1.1 & 1.1 & 1.1 \\
\hline Public works & 4.0 & 5.5 & 5.8 & 6.2 & 6.6 & 6.9 & 7.2 & 7.6 & 8.1 & 8.3 & 8.6 & 8.8 & 8.9 \\
\hline Transport & 0.5 & 0.7 & 0.8 & 0.8 & 0.9 & 0.9 & 1.0 & 1.0 & 1.1 & 1.1 & 1.2 & 1.2 & 1.2 \\
\hline Electricity, water and mines & 0.6 & 0.8 & 0.9 & 0.9 & 1.0 & 1.0 & 1.1 & 1.2 & 1.2 & 1.3 & 1.3 & 1.3 & 1.3 \\
\hline Urban planning and housing & 1.1 & 1.5 & 1.6 & 1.7 & 1.8 & 1.9 & 1.9 & 2.1 & 2.2 & 2.2 & 2.3 & 2.4 & 2.4 \\
\hline Towns & 0.6 & 0.8 & 0.9 & 0.9 & 1.0 & 1.0 & 1.1 & 1.1 & 1.2 & 1.2 & 1.3 & 1.3 & 1.3 \\
\hline Rural sector & 2.9 & 3.7 & 3.9 & 4.0 & 4.2 & 5.8 & 6.0 & 6.2 & 6.4 & 6.6 & 6.8 & 6.9 & 7.0 \\
\hline Agriculture & 1.9 & 2.4 & 2.5 & 2.6 & 2.7 & 3.7 & 3.9 & 4.0 & 4.2 & 4.3 & 4.4 & 4.5 & 4.6 \\
\hline $\begin{array}{l}\text { Stockbreeding, fisheries and animal } \\
\text { industries }\end{array}$ & 0.5 & 0.7 & 0.7 & 0.7 & 0.8 & 1.1 & 1.1 & 1.1 & 1.2 & 1.2 & 1.2 & 1.3 & 1.3 \\
\hline Forests and environment & 0.5 & 0.6 & 0.6 & 0.7 & 0.7 & 1.0 & 1.0 & 1.0 & 1.1 & 1.1 & 1.1 & 1.2 & 1.2 \\
\hline Other Ministries and Institutions & 21.6 & 21.8 & 23.6 & 24.0 & 23.6 & 23.4 & 22.1 & 23.3 & 23.3 & 23.3 & 23.9 & 25.5 & 25.9 \\
\hline Total Ministerial Budget & 100 & 100 & 100 & 100 & 100 & 100 & 100 & 100 & 100 & 100 & 100 & 100 & 100 \\
\hline
\end{tabular}




\section{Annex 6.1 : EDUCATION}

\section{ANNEXE 6.1.1 : MTEF OF EDUCATION SECTOR STRATEGY/MDG}

\begin{tabular}{|c|c|c|c|c|c|c|c|c|c|}
\hline Scenario : aligning education strategy objectives and MDG & 2003 & 2004 & 2005 & 2006 & 2007 & 2008 & 2009 & 2010 & 2011 \\
\hline Number of teacher to be recruited in primary & 2534 & 3401 & 3658 & 2954 & 2152 & 2249 & 2352 & 2459 & 2572 \\
\hline Number of classroom to be build in primary & 2534 & 3401 & 3658 & 2954 & 2152 & 2249 & 2352 & 2459 & 2572 \\
\hline Total expenditure "Scenario : aligning education strategy/MDG " & 214871 & 248995 & 279777 & 303726 & 329898 & 370006 & 408884 & 452980 & 503116 \\
\hline Financing & 199624 & 218595 & 240776 & 267053 & 291316 & 315345 & 350376 & 390999 & 438216 \\
\hline Internal & 195400 & 204800 & 225100 & 248700 & 276300 & 309600 & 340800 & 381700 & 420000 \\
\hline External & 4224 & 13795 & 15676 & 18353 & 15016 & 5745 & 9576 & 9299 & 18216 \\
\hline Pare (Education reform support program (ERSP) & 333 & 333 & 333 & 0 & 0 & 0 & 0 & 0 & 0 \\
\hline Japanese grant & 0 & 6655 & 6972 & 6972 & 5472 & 0 & 0 & 0 & 0 \\
\hline Education II & 2125 & 2125 & 0 & 0 & 0 & 0 & 0 & 0 & 0 \\
\hline UNICEF & 717 & 780 & 806 & 824 & 823 & 0 & 0 & 0 & 0 \\
\hline Others & 1049 & 3902 & 7564 & 10557 & 8721 & 5745 & 9576 & 9299 & 18216 \\
\hline Gap & 15247 & 30400 & 39001 & 36673 & 38582 & 54661 & 58508 & 61981 & 64900 \\
\hline
\end{tabular}

ANNEX 6.1.2 : MTEF OF EDUCATION SECTOR STRATEGY/PRSP BASE CASE

\begin{tabular}{|c|c|c|c|c|c|c|c|c|c|}
\hline Scenario : Aligning the Education sector strategy with the PRSP base case & 2003 & 2004 & 2005 & 2006 & 2007 & 2008 & 2009 & 2010 & 2011 \\
\hline Number of teacher to be recruited in primary & 1879 & 2629 & 2805 & 2993 & 2613 & 2062 & 2156 & 2254 & 2357 \\
\hline Number of classroom to be build in primary & 1879 & 2629 & 2805 & 2993 & 2613 & 2062 & 2156 & 2254 & 2357 \\
\hline Total expenditure "Scenario alignement with PRSP macro framework & 199624 & 218595 & 240775 & 267052 & 291316 & 315345 & 350376 & 390999 & 438216 \\
\hline Financing & 199624 & 218595 & 240775 & 267052 & 291316 & 315345 & 350376 & 390999 & 438216 \\
\hline Internal & 195400 & 204800 & 225100 & 248700 & 276300 & 309600 & 340800 & 381700 & 420000 \\
\hline External & 4224 & 13795 & 15676 & 18353 & 15016 & 5745 & 9576 & 9299 & 18216 \\
\hline Pare (Education reform support program (ERSP) & 333 & 333 & 333 & 0 & 0 & 0 & 0 & 0 & 0 \\
\hline Japanese grant & 0 & 6655 & 6972 & 6972 & 5472 & 0 & 0 & 0 & 0 \\
\hline Education II & 2125 & 2125 & 0 & 0 & 0 & 0 & 0 & 0 & 0 \\
\hline UNICEF & 717 & 780 & 806 & 824 & 823 & 0 & 0 & 0 & 0 \\
\hline Others & 1049 & 3902 & 7564 & 10557 & 8721 & 5745 & 9576 & 9299 & 18216 \\
\hline Gap & $\mathbf{0}$ & $\mathbf{0}$ & $\mathbf{0}$ & $\mathbf{0}$ & $\mathbf{0}$ & $\mathbf{0}$ & $\mathbf{0}$ & $\mathbf{0}$ & $\mathbf{0}$ \\
\hline
\end{tabular}


Annex

\begin{tabular}{|c|c|c|c|c|c|c|c|c|c|c|}
\hline $\mathbf{N}^{\circ}$ & PROGRAM & $\mathbf{N}^{\circ}$ & \# & PROJECT & 2003 & 2004 & 2005 & 2006 & 2007 & $\begin{array}{l}\text { TOTAL } \\
\text { 2003-07 } \\
\end{array}$ \\
\hline \multirow{6}{*}{1.} & \multirow{6}{*}{$\begin{array}{l}\text { SUPPLY OF \& ACCESS TO } \\
\text { EDUCATION }\end{array}$} & 1.1 & 1 & Human resources & 130.693 & 132.694 & 133.396 & 135.508 & 137.556 & 669.847 \\
\hline & & 1.2 & 2 & Services operation and maintenance & 30.139 & 31.410 & 32.760 & 34.195 & 35.721 & 164.225 \\
\hline & & 1.3 & 3 & Fostering child care support & 3.974 & 4.329 & 4.718 & 3.965 & 4.565 & 21.551 \\
\hline & & 1.4 & 4 & Increasing access to primary education & 22.512 & 38.706 & 52.265 & 76.784 & 93.012 & 283.279 \\
\hline & & 1.5 & 5 & Increasing access to secondary education & 709 & 647 & 633 & 633 & 240 & 2.862 \\
\hline & & & & TOTAL (PROGRAM) & 188.027 & 207.787 & 223.772 & 251.085 & 271.094 & 1.141 .764 \\
\hline \multirow{6}{*}{2.} & \multirow{6}{*}{ EDUCATION QUALITY } & 2.1 & 6 & Decrease school drop out (by reducing repeaters) & 2.389 & 2.741 & 3.145 & 3.910 & 6.964 & 19.149 \\
\hline & & 2.2 & 7 & Improve the quality of educational structure & 2.449 & 2.460 & 2.453 & 2.467 & 2.630 & 12.458 \\
\hline & & 2.3 & 8 & Revise education curricula & 1.261 & 796 & 1.001 & 898 & 950 & 4.906 \\
\hline & & 2.4 & 9 & $\begin{array}{l}\text { Facilitating access to and availability of textbooks and } \\
\text { educational material }\end{array}$ & 3.027 & 3.019 & 2.691 & 2.691 & 3.030 & 14.458 \\
\hline & & 2.5 & 10 & Improving health and combating HIV/AIDS and STD in school & 586 & 570 & 340 & 357 & 368 & 2.220 \\
\hline & & & & TOTAL (PROGRAM) & 9.712 & 9.585 & 9.629 & 10.323 & 13.942 & 53.192 \\
\hline \multirow{5}{*}{3.} & \multirow{5}{*}{ PARTNERSHIP } & 3.1 & 11 & Developing participatory management school facilities & 0 & 0 & 0 & 0 & 0 & $\mathbf{0}$ \\
\hline & & 3.2 & 12 & $\begin{array}{l}\text { Involving socio-professional association in designing technical, } \\
\text { technological \& professional training program }\end{array}$ & 0 & 0 & 0 & 0 & 0 & $\mathbf{0}$ \\
\hline & & 3.3 & 13 & Defining \& implementing national private education policies & 0 & 0 & 6.000 & 4.000 & 4.000 & 14.000 \\
\hline & & 3.4 & 14 & $\begin{array}{l}\text { Defining and promoting Government/Private education } \\
\text { partnership }\end{array}$ & 74 & 29 & 33 & 38 & 50 & 225 \\
\hline & & & & TOTAL (PROGRAMM) & 74 & 29 & 6.033 & 4.038 & 4.050 & 14.225 \\
\hline \multirow{4}{*}{4.} & \multirow{4}{*}{$\begin{array}{l}\text { MANAGEMENT AND } \\
\text { GOVERNANCE }\end{array}$} & 4.1 & 15 & Making education regional structures accountable & 225 & 225 & 225 & 225 & 225 & 1.125 \\
\hline & & 4.2 & 16 & Improving information system & 614 & 695 & 1.100 & 1.366 & 1.990 & 5.765 \\
\hline & & 4.3 & 17 & Promoting good governance & 972 & 274 & 15 & 15 & 15 & 1.291 \\
\hline & & & & TOTAL (PROGRAMM) & 1.811 & 1.194 & 1.340 & 1.606 & 2.230 & 8.181 \\
\hline 5. & GRAND TOTAL & & & & 199.624 & 218.595 & 240.775 & 267.052 & 291.316 & 1.217 .362 \\
\hline \multirow{8}{*}{6.} & \multirow{8}{*}{$\begin{array}{l}\text { FINANCING } \\
\text { TOTAL }\end{array}$} & & & INTERNAL RESOURCES + HIPC & 195400 & 204800 & 225100 & 248700 & 276300 & 1150300 \\
\hline & & & & EXTERNAL FINANCING & 4264 & 15675 & 15.675 & 18352 & 15016 & 67062 \\
\hline & & & & ERSP & 333 & 333 & 333 & 0 & 0 & 999 \\
\hline & & & & Japanese grant & 0 & 6.655 & 6.972 & 6.972 & 5.472 & 26.071 \\
\hline & & & & Education II & 2.125 & 2.25 & 0 & 0 & 0 & 4.250 \\
\hline & & & & UNICEF & 717 & 780 & 806 & 824 & 823 & 3.950 \\
\hline & & & & Others & 1.049 & 3.902 & 7.564 & 10.556 & 8.721 & 31.792 \\
\hline & & & & GRANT TOTAL financing & 199.624 & 218.595 & 240.775 & 267.052 & 291.316 & 1.217 .362 \\
\hline 7. & FINANCING GAP (SI & LUS) & & & $\mathbf{0}$ & $\mathbf{0}$ & $\mathbf{0}$ & $\mathbf{0}$ & $\mathbf{0}$ & $\mathbf{0}$ \\
\hline
\end{tabular}




\section{Annex 6.2 : HEALTH}

ANNEX 6.2.1 : MTEF OF HEALTH SECTOR STRATEGY/MDG

\begin{tabular}{|c|c|c|c|c|c|c|c|c|c|}
\hline \multirow[t]{2}{*}{$\mathbf{N}^{\circ}$} & \multirow[t]{2}{*}{ PROGRAM } & \multicolumn{2}{|c|}{$\mathbf{N}^{\circ}$} & \multicolumn{6}{|c|}{ In millions of FCFA } \\
\hline & & & PROJECT & 2003 & 2004 & 2005 & 2006 & 2007 & Total \\
\hline \multirow{15}{*}{1.} & \multirow{15}{*}{ Diseases Control } & 1.1 & Malaria control & 4646 & 18521 & 12708 & 7500 & 8000 & $\mathbf{5 1 3 7 5}$ \\
\hline & & 1.2 & Leprosies control & 48 & 100 & 127 & 246 & 299 & 820 \\
\hline & & 1.3 & River blindness control & 675 & 709 & 744 & 781 & 820 & 3730 \\
\hline & & 1.4 & Trypanosomiasis control & 147 & 224 & 285 & 606 & 738 & 2000 \\
\hline & & 1.5 & Guinea worm control & 85 & 130 & 165 & 393 & 478 & 1251 \\
\hline & & 1.6 & Buruli ulcer control & 110 & 190 & 242 & 479 & 583 & 1604 \\
\hline & & 1.7 & Non transmissible diseases control & 920 & 1500 & 1620 & 1733 & 1872 & 7645 \\
\hline & & 1.8 & AIDS and STI control & 15515 & 29748 & 35246 & 32882 & 34070 & 147461 \\
\hline & & 1.9 & Tuberculosis control & 662 & 1539 & 1577 & 1778 & 1867 & 7423 \\
\hline & & 1.10 & Blindness control & 80 & 122 & 200 & 325 & 396 & 1123 \\
\hline & & 1.11 & Schistosomiasis control & 42 & 179 & 228 & 429 & 522 & 1400 \\
\hline & & 1.12 & Dental diseases control & 10 & 125 & 159 & 330 & 402 & 1026 \\
\hline & & 1.13 & Deafness control & 30 & 46 & 58 & 153 & 186 & 473 \\
\hline & & 1.14 & Government care of emergency, epidemic and disaster & 90 & 268 & 341 & 793 & 965 & 2458 \\
\hline & & & Total (program) & 23060 & 53400 & 53702 & 48429 & 51200 & 229791 \\
\hline & \multirow{4}{*}{$\begin{array}{l}\text { Maternal, child } \\
\text { and elderly } \\
\text { health }\end{array}$} & 2.1 & Maternal, adolescent, and elderly care & 60 & 350 & 446 & 590 & 750 & 2196 \\
\hline & & 2.2 & Expanded Programme on immunization ( EPI ) & 2342 & 3514 & 3935 & 4407 & 4936 & 19135 \\
\hline & & 2.3 & IMCI (Integrated management of childhood illness) & 7 & 75 & 96 & 122 & 250 & 549 \\
\hline & & & Total (program) & 2409 & 3939 & 4476 & 5119 & 5936 & 21879 \\
\hline \multirow{5}{*}{3.} & \multirow{5}{*}{$\begin{array}{l}\text { Health } \\
\text { promotion }\end{array}$} & 3.1 & Health promotion through nourishment and nutrition & 105 & 500 & 650 & 650 & 750 & 2655 \\
\hline & & 3.2 & Mental health and human behavior & 68 & 150 & 400 & 450 & 650 & 1718 \\
\hline & & 3.3 & Water, hygiene and sanitation & 100 & 153 & 450 & 544 & 662 & 1909 \\
\hline & & 3.4 & Promoting traditional medicine & 12 & 80 & 175 & 182 & 222 & 671 \\
\hline & & & Total (program) & 285 & 883 & 1675 & 1826 & 2284 & 6952 \\
\hline \multirow{3}{*}{4.} & \multirow{3}{*}{$\begin{array}{l}\text { Enhancing local } \\
\text { communities } \\
\text { response } \\
\text { /partnership }\end{array}$} & 4.1 & $\begin{array}{l}\text { Support to local communities (consultative structures, capacity building, } \\
\text { training) }\end{array}$ & 50 & 100 & 127 & 272 & 299 & 849 \\
\hline & & 4.3 & Promoting health insurance schemes & 100 & 350 & 420 & 504 & 605 & 1979 \\
\hline & & & Total (program) & 150 & 450 & 547 & 776 & 904 & 2827 \\
\hline \multirow{6}{*}{5.} & \multirow{6}{*}{$\begin{array}{l}\text { Essential drugs } \\
\text { and medical } \\
\text { supplies }\end{array}$} & 5.1 & Subsidies to priority programs (AIDS/ RBM/Tb) & 135 & 350 & 455 & 592 & 769 & 2300 \\
\hline & & 5.2 & Others drugs/medicine procurement & 801 & 881 & 1145 & 1260 & 1638 & 5725 \\
\hline & & 5.3 & SYNAME (Regulation/supervision/regulation) & 50 & 76 & 97 & 118 & 135 & 476 \\
\hline & & 5.6 & Drug management training & 164 & 100 & 105 & 110 & 127 & 606 \\
\hline & & 5.7 & Biological screening (cost containment) & 50 & 421 & 536 & 330 & 402 & 1739 \\
\hline & & & Total (program) & 1200 & 1828 & 2339 & 2409 & 3071 & 10847 \\
\hline \multirow{7}{*}{6.} & \multirow{7}{*}{$\begin{array}{l}\text { Strengthening } \\
\text { infrastructure } \\
\text { and equipment } \\
\text { capacities }\end{array}$} & 6.1 & Rehabilitation/equipment provincial hospitals (09) & 1200 & 2500 & 3350 & 3600 & 5000 & 15650 \\
\hline & & 6.2 & Rehabilitation/construction/equipment of district hospitals (15 per year) & 3190 & 3669 & 4219 & 4852 & 5579 & 21508 \\
\hline & & 6.3 & Rehabilitation/construction/equipment CSI & 8394 & 17453 & 19198 & 18430 & 21195 & 84671 \\
\hline & & 6.4 & Construction and equipment of administrative services & 1370 & 2089 & 2661 & 2474 & 3051 & 11645 \\
\hline & & 6.5 & Housing construction in difficult areas 141 (HIPC) & 5448 & & & & & 5448 \\
\hline & & 6.6 & Study of district hospitals rehabilitation and construction requirements & 803 & & & & & 803 \\
\hline & & & Total (program) & 20405 & 25711 & 29428 & 29357 & 34825 & 139725 \\
\hline
\end{tabular}




\begin{tabular}{|c|c|c|c|c|c|c|c|c|c|}
\hline \multirow[t]{2}{*}{$\mathbf{N}^{\circ}$} & \multirow[t]{2}{*}{ PROGRAM } & \multicolumn{2}{|c|}{$\mathbf{N}^{\circ}$} & \multicolumn{6}{|c|}{ In millions of FCFA } \\
\hline & & & PROJECT & 2003 & 2004 & 2005 & 2006 & 2007 & Total \\
\hline \multirow{7}{*}{7.} & \multirow{7}{*}{$\begin{array}{l}\text { Human } \\
\text { development } \\
\text { resources }\end{array}$} & 7.1 & Personnel expenditure excluding specialists & 21337 & 22025 & 22906 & 23937 & 25374 & 115580 \\
\hline & & 7.2 & Recruitment specialized doctors & 250 & 381 & 486 & 588 & 716 & 2421 \\
\hline & & 7.4 & Others personnel /incentives expenditure & 263 & 401 & 511 & 619 & 753 & 2547 \\
\hline & & 7.5 & Training, scholarship and internship & 440 & 671 & 1023 & 1561 & 2380 & 6076 \\
\hline & & 7.6 & Human resources management (SIGIPES) & 779 & 700 & 650 & 600 & 550 & 3279 \\
\hline & & 7.7 & HIPC special recruitment & 3057 & 3057 & 3057 & 3057 & 3057 & 15285 \\
\hline & & & Total (program) & 26126 & 27236 & 28633 & 30362 & 32830 & 145188 \\
\hline \multirow{4}{*}{9.} & \multirow{4}{*}{$\begin{array}{l}\text { Work } \\
\text { environment }\end{array}$} & 9.1 & Service operations & 17589 & 21000 & 22000 & 23000 & 26000 & 109589 \\
\hline & & 9.2 & Building maintenance & 964 & 1500 & 1500 & 1800 & 2750 & 8514 \\
\hline & & 9.3 & Logistics procurement & 900 & 1373 & 1748 & 2118 & 2578 & 8716 \\
\hline & & & Total (program) & 19453 & 23873 & 25248 & 26918 & 31328 & 126819 \\
\hline \multirow{3}{*}{10.} & \multirow{3}{*}{$\begin{array}{l}\text { Partnership } \\
\text { development }\end{array}$} & 10.1 & Outsourcing with private sector/NGO/other organizations & 85 & 130 & 165 & 200 & 243 & 823 \\
\hline & & 10.2 & Supporting non commercial private healthcare & 500 & 763 & 824 & 889 & 961 & 3936 \\
\hline & & & Total (program) & 585 & 892 & 989 & 1089 & 1204 & 4759 \\
\hline \multirow{6}{*}{11.} & \multirow{6}{*}{$\begin{array}{l}\text { Improving } \\
\text { management }\end{array}$} & 11.1 & Establishing a pricing scheme /Monitoring and evaluation & 70 & 107 & 136 & 165 & 201 & 678 \\
\hline & & 11.2 & Improving absorption capacity & 200 & 305 & 388 & 471 & 573 & 1937 \\
\hline & & 11.3 & Supporting planning /programming & 50 & 100 & 150 & 182 & 221 & 703 \\
\hline & & 11.4 & Supporting the health information system & 250 & 381 & 486 & 588 & 716 & 2421 \\
\hline & & 11.5 & Audit system & 50 & 76 & 97 & 118 & 143 & 484 \\
\hline & & & Total (program) & 620 & 969 & 1257 & 1523 & 1854 & 6223 \\
\hline \multirow{4}{*}{12.} & \multirow{4}{*}{ Structural reform } & 12.1 & Revising organizational arrangements and decentralization & 150 & 229 & 291 & 353 & 430 & 1453 \\
\hline & & 12.2 & Hospital reform & 200 & 305 & 388 & 471 & 573 & 1937 \\
\hline & & 12.3 & Developing a multisectoral approach & 50 & 76 & 97 & 118 & 143 & 484 \\
\hline & & & Total (program) & 400 & 610 & 777 & 941 & 1146 & 3874 \\
\hline \multirow{9}{*}{13.} & \multirow{9}{*}{$\begin{array}{l}\text { Subsidies toward } \\
\text { modernizing } \\
\text { government } \\
\text { owned facilities }\end{array}$} & 13.1 & Hôpital général de Douala & 300 & 450 & 573 & 694 & 845 & 2863 \\
\hline & & 13.2 & Hôpital général de Yaoundé & 300 & 450 & 573 & 694 & 845 & 2863 \\
\hline & & 13.3 & Centre Hospitalier Universitaire de Yaoundé & 300 & 450 & 573 & 694 & 845 & 2863 \\
\hline & & 13.4 & Centre Pasteur de Yaoundé & 120 & 180 & 229 & 278 & 338 & 1145 \\
\hline & & 13.5 & Hôpital Pédiatrique et Gynéco obstétrique de Yaoundé & 400 & 600 & 764 & 926 & 1127 & 3817 \\
\hline & & 13.6 & LANACOME & 100 & 200 & 255 & 309 & 376 & 1239 \\
\hline & & 13.7 & Subsidies to CENAME & 100 & 140 & 178 & 216 & 263 & 897 \\
\hline & & 13.8 & National Health Observatory & 280 & 427 & 544 & 659 & 802 & 2712 \\
\hline & & & Total (program) & 1900 & 2897 & 3689 & 4470 & 5441 & 18397 \\
\hline \multirow{4}{*}{14.} & \multirow{4}{*}{$\begin{array}{l}\text { Operation and } \\
\text { investment credit } \\
\text { to second } \\
\text { category } \\
\text { hospitals }\end{array}$} & 14.1 & Hôpital Central de Yaoundé & 175 & 267 & 340 & 412 & 501 & 1695 \\
\hline & & 14.2 & Hôpital Laquintinie & 175 & 267 & 340 & 412 & 501 & 1695 \\
\hline & & 14.3 & Hôpital Jamot & 150 & 229 & 291 & 353 & 430 & 1453 \\
\hline & & & Total (program) & 500 & 763 & 971 & 1177 & 1432 & 4842 \\
\hline
\end{tabular}




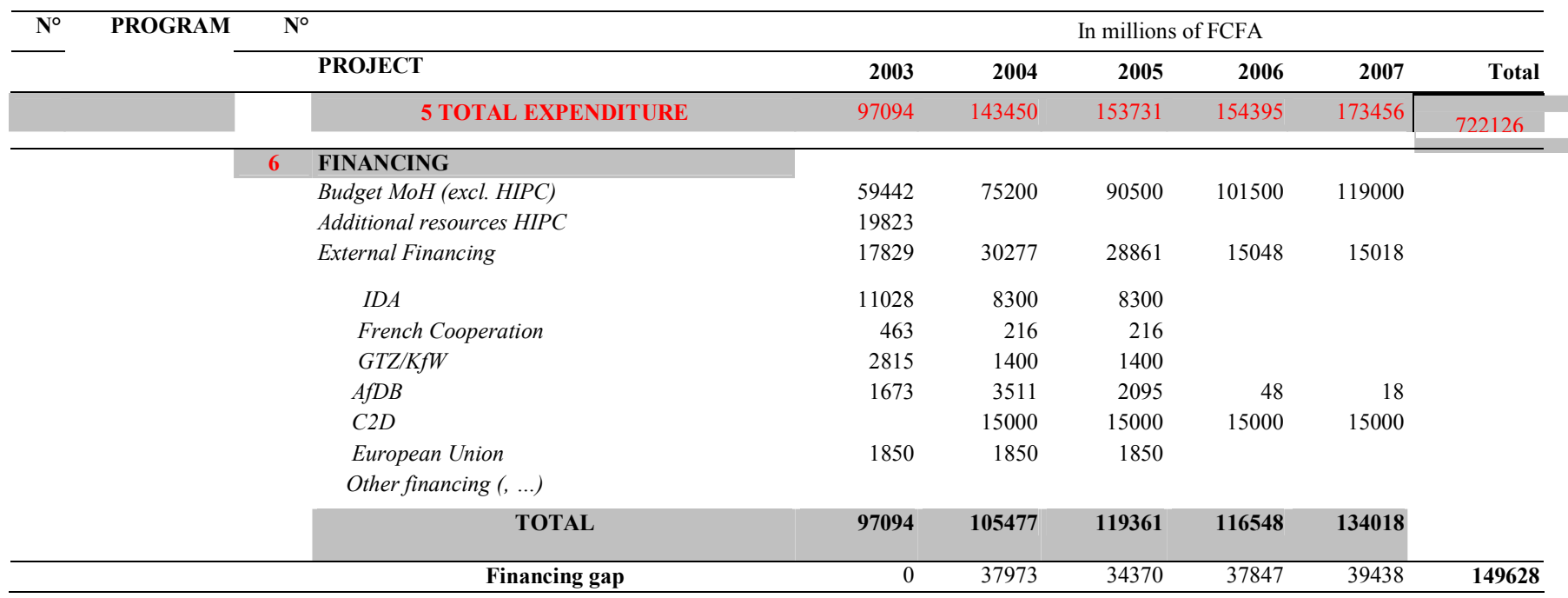




\section{ANNEX 6.2.2 : MTEF OF HEALTH SECTOR STRATEGY/PRSP BASE CASE}

\begin{tabular}{|c|c|c|c|c|c|c|c|c|c|}
\hline \multicolumn{10}{|c|}{ MEDIUM TERM EXPENDITURE FRAMEWORK 2003-2007 } \\
\hline & & & & 2003 & 2004 & 2005 & 2006 & 2007 & Total \\
\hline $\mathbf{N}^{\circ}$ & PROGRAMS & $\mathbf{N}^{\circ}$ & PROJECT & (millions) & & & & & \\
\hline \multirow{15}{*}{1.} & \multirow{15}{*}{ Disease control } & 1.1 & Malaria control & 4,646 & 5,808 & 7,550 & 9,060 & 9,513 & 36,576 \\
\hline & & 1.2 & Leprosies control & 48 & 100 & 127 & 246 & 299 & 820 \\
\hline & & 1.3 & River blindness control & 675 & 709 & 744 & 781 & 820 & 3,730 \\
\hline & & 1.4 & Trypanosomiasis control & 147 & 224 & 285 & 606 & 738 & 2,000 \\
\hline & & 1.5 & Guinea worm control & 85 & 130 & 165 & 393 & 478 & 1,251 \\
\hline & & 1.6 & Buruli ulcer control & 110 & 190 & 242 & 479 & 583 & 1,604 \\
\hline & & 1.7 & Non transmissible diseases control & 920 & 1,500 & 1,620 & 1,733 & 1,872 & $\mathbf{7 , 6 4 5}$ \\
\hline & & 1.8 & AIDS and STI control & 15,515 & 16,757 & 19,270 & 20,234 & 21,245 & 93,021 \\
\hline & & 1.9 & Tuberculosis control & 662 & 795 & 954 & 1,001 & 1,051 & $\mathbf{4 , 4 6 3}$ \\
\hline & & 1,10 & Blindness control & 80 & 122 & 200 & 325 & 396 & 1,123 \\
\hline & & 1.11 & Schistosomiasis control & 42 & 179 & 228 & 429 & 522 & 1,400 \\
\hline & & 1.12 & Dental diseases control & 10 & 125 & 159 & 330 & 402 & 1,026 \\
\hline & & 1.13 & Deafness control & 30 & 46 & 58 & 153 & 186 & 473 \\
\hline & & 1.14 & $\begin{array}{l}\text { Government care of emergency, } \\
\text { epidemic and disaster }\end{array}$ & 90 & 268 & 341 & 793 & 965 & 2,458 \\
\hline & & & Total (program) & 23,060 & 26,951 & 31,944 & 36,563 & 39,072 & 157,590 \\
\hline \multirow{4}{*}{2.} & \multirow{4}{*}{$\begin{array}{l}\text { Maternal, child } \\
\text { and elderly } \\
\text { health }\end{array}$} & 2.1 & Maternal, adolescent, and elderly care & 60 & 350 & 446 & 590 & 750 & 2,196 \\
\hline & & 2.2 & $\begin{array}{l}\text { Expanded Programme on immunization } \\
\text { ( EPI ) }\end{array}$ & 2,342 & 2,928 & 3,279 & 3,771 & 4,224 & 16,544 \\
\hline & & 2.3 & $\begin{array}{l}\text { IMCI (Integrated management of } \\
\text { childhood illness) }\end{array}$ & 7 & 75 & 96 & 122 & 250 & 549 \\
\hline & & & Total (program) & 2,409 & 3,353 & 3,821 & 4,483 & 5,224 & 19,289 \\
\hline \multirow{5}{*}{3.} & \multirow{5}{*}{$\begin{array}{l}\text { Health } \\
\text { promotion }\end{array}$} & 3.1 & $\begin{array}{l}\text { Health promotion through nourishment } \\
\text { and nutrition }\end{array}$ & 105 & 500 & 650 & 1,514 & 1,550 & $\overline{4,319}$ \\
\hline & & 3.2 & Mental health and human behavior & 68 & 150 & 400 & 450 & 650 & 1,718 \\
\hline & & 3.3 & Water, hygiene and sanitation & 100 & 153 & 450 & 544 & 662 & 1,909 \\
\hline & & 3.4 & Promoting traditional medicine & 12 & 80 & 175 & 182 & 416 & 865 \\
\hline & & & Total (program) & 285 & 883 & 1,675 & 2,690 & 3,278 & 8,810 \\
\hline \multirow{3}{*}{4.} & \multirow{3}{*}{$\begin{array}{l}\text { Enhancing local } \\
\text { communities } \\
\text { response } \\
\text { /partnership }\end{array}$} & 4.1 & $\begin{array}{l}\text { Support to local communities } \\
\text { (consultative structures, capacity }\end{array}$ & 50 & 100 & 127 & 272 & 299 & 849 \\
\hline & & 4.3 & Promoting health insurance schemes & 100 & 350 & 420 & 504 & 605 & 1,979 \\
\hline & & & Total (program) & 150 & 450 & 547 & 776 & 904 & 2,827 \\
\hline \multirow{6}{*}{5.} & \multirow{6}{*}{$\begin{array}{l}\text { Essential drugs } \\
\text { and medical } \\
\text { supplies }\end{array}$} & 5.1 & $\begin{array}{l}\text { Subsidies to priority programs (AIDS/ } \\
\mathrm{RBM} / \mathrm{Tb} \text { ) }\end{array}$ & 135 & 350 & 455 & 592 & 769 & $\overline{2,300}$ \\
\hline & & 5.2 & Others drugs/medicine procurement & 801 & 881 & 1,145 & 1,260 & 1,638 & 5,725 \\
\hline & & 5.3 & $\begin{array}{l}\text { SYNAME } \\
\text { (Regulation/supervision/regulation }\end{array}$ & 50 & 76 & 97 & 118 & 135 & 476 \\
\hline & & 5.6 & Drug management training & 164 & 100 & 105 & 110 & 127 & 606 \\
\hline & & 5.7 & Biological screening (cost containment) & 50 & 421 & 536 & 330 & 402 & 1,739 \\
\hline & & & Total (program) & 1,200 & 1,828 & 2,339 & 2,409 & 3,071 & 10,847 \\
\hline
\end{tabular}




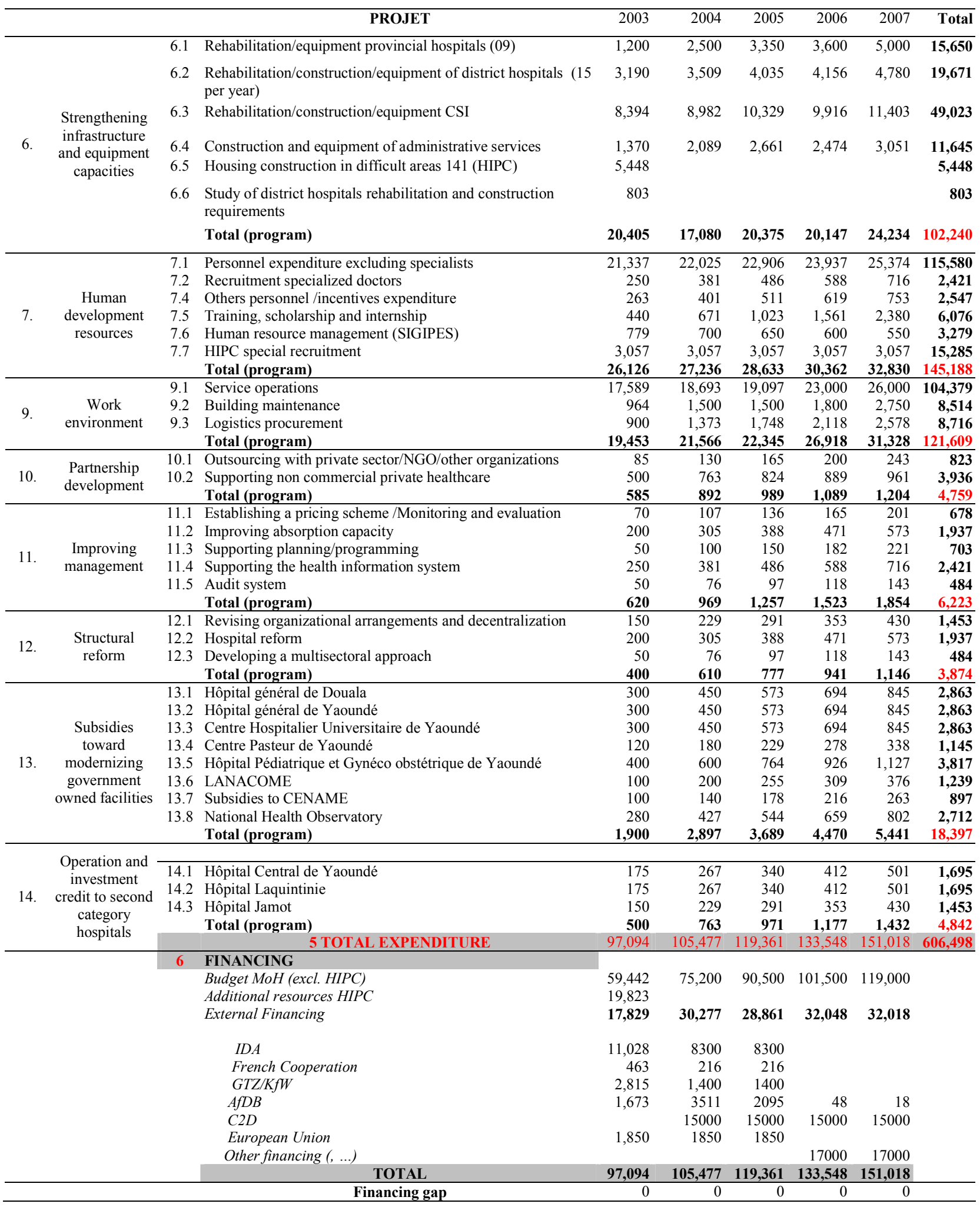


Annex

Annex 6.3: PUBLIC WORKS

ANNEX 6.3.1: MEDIUM-TERM EXPENDITURE FRAMEWORK (MTEF) FOR THE PUBLIC WORKS SECTOR STRATEGY/MDGS

\begin{tabular}{|c|c|c|c|c|c|c|c|c|c|c|c|c|c|c|c|c|}
\hline \multicolumn{17}{|c|}{ MDGS OPTIONS } \\
\hline \multirow[t]{3}{*}{ No. } & Year & $\begin{array}{c}\text { Type of } \\
\text { roads }\end{array}$ & \multicolumn{6}{|c|}{ Linear (in $\mathrm{km}$ ) } & \multicolumn{3}{|c|}{ Costs } & \multicolumn{5}{|r|}{$\%$} \\
\hline & & & \multicolumn{3}{|c|}{ Maintenance } & \multicolumn{3}{|c|}{ Investment } & \multicolumn{3}{|c|}{ Maintenance } & \multicolumn{3}{|c|}{ Investment } & Annual total & $\begin{array}{c}\text { In good } \\
\text { condition }\end{array}$ \\
\hline & & & $\begin{array}{c}\text { In } \\
\text { progress }\end{array}$ & $\begin{array}{c}\text { Periodi } \\
\mathrm{c}\end{array}$ & Total & $\begin{array}{c}\text { Rehabilitati } \\
\text { on }\end{array}$ & $\begin{array}{c}\text { New } \\
\text { works }\end{array}$ & Total & $\begin{array}{c}\text { In } \\
\text { progress 1/ }\end{array}$ & $\begin{array}{c}\text { Periodic } \\
2 / \\
\end{array}$ & Total & $\begin{array}{c}\text { Rehabilita } \\
\text { tion 3/ }\end{array}$ & $\begin{array}{c}\text { New works } \\
4 /\end{array}$ & & Total & \\
\hline \multirow{5}{*}{1.} & & Asphalt & $3,839.0$ & 289.0 & $4,128.0$ & 140.0 & 544.0 & 684.0 & $6,910.2$ & $4,566.2$ & $11,476.4$ & $16,212.0$ & $136,000.0$ & $152,212.0$ & $163,688.4$ & 94.0 \\
\hline & & Dirt & $6,680.0$ & $2,000.0$ & $8,680.0$ & 613.0 & - & 613.0 & $10,688.0$ & $22,600.0$ & $33,288.0$ & $70,985.4$ & - & $\begin{array}{r}-70,985.4 \\
\end{array}$ & $104,273.4$ & 77.7 \\
\hline & 2003 & R.P. & 475.0 & 550.0 & $1,025.0$ & 345.0 & - & 345.0 & 760.0 & $3,300.0$ & $4,060.0$ & $39,951.0$ & & - $39,951.0$ & $44,011.0$ & 6.8 \\
\hline & & N.P. & & & & $1,691.0$ & - & $1,691.0$ & & & & $195,817.8$ & & $195,817.8$ & $195,817.8$ & 7.0 \\
\hline & Total 1 & & $10,994.0$ & $2,839.0$ & $13,833.0$ & $2,789.0$ & 544.0 & $3,333.0$ & $18,358.2$ & $30,466.2$ & $48,824.4$ & $322,966.2$ & $136,000.0$ & $458,966.2$ & $507,790.6$ & \\
\hline \multirow{5}{*}{2.} & & Asphalt & $4,383.0$ & 289.0 & $4,672.0$ & 140.0 & 544.0 & 684.0 & $7,889.4$ & $4,566.2$ & $12,455.6$ & $16,212.0$ & $136,000.0$ & $152,212.0$ & $164,667.6$ & 94.6 \\
\hline & & Dirt & $6,136.0$ & $2,000.0$ & $8,136.0$ & 613.0 & - & 613.0 & $9,817.6$ & $22,600.0$ & $32,417.6$ & $70,985.4$ & - & - $\quad 70,985.4$ & $103,403.0$ & 76.3 \\
\hline & 2004 & R.P. & $1,370.0$ & 550.0 & $1,920.0$ & 345.0 & - & 345.0 & $2,192.0$ & $3,300.0$ & $5,492.0$ & $39,951.0$ & & - $39,951.0$ & $45,443.0$ & 14.2 \\
\hline & & N.P. & $1,691.0$ & & $1,691.0$ & $1,691.0$ & - & $1,691.0$ & $2,705.6$ & & $2,705.6$ & $195,817.8$ & & $195,817.8$ & $198,523.4$ & 14.1 \\
\hline & Total 2 & & $13,580.0$ & $2,839.0$ & $16,419.0$ & $2,789.0$ & 544.0 & $3,333.0$ & $22,604.6$ & $30,466.2$ & $53,070.8$ & $322,966.2$ & $136,000.0$ & $458,966.2$ & $512,037.0$ & \\
\hline \multirow{5}{*}{3.} & & Asphalt & $4,927.0$ & 289.0 & $5,216.0$ & 140.0 & 544.0 & 684.0 & $8,868.6$ & $4,566.2$ & $13,434.8$ & $16,212.0$ & $136,000.0$ & $152,212.0$ & $165,646.8$ & 95.1 \\
\hline & & Dirt & $5,592.0$ & $2,000.0$ & $7,592.0$ & 613.0 & - & 613.0 & $8,947.2$ & $22,600.0$ & $31,547.2$ & $70,985.4$ & - & $\begin{array}{r}70,985.4 \\
-\end{array}$ & $102,532.6$ & 74.8 \\
\hline & 2005 & R.P. & $2,265.0$ & 550.0 & $2,815.0$ & 345.0 & - & 345.0 & $3,624.0$ & $3,300.0$ & $6,924.0$ & $39,951.0$ & & - $39,951.0$ & $46,875.0$ & 21.6 \\
\hline & & N.P. & $3,382.0$ & & $3,382.0$ & $1,691.0$ & - & $1,691.0$ & $5,411.2$ & - & $5,411.2$ & $195,817.8$ & & $195,817.8$ & $201,229.0$ & 21.1 \\
\hline & Total 3 & & $16,166.0$ & $2,839.0$ & $19,005.0$ & $2,789.0$ & 544.0 & $3,333.0$ & $26,851.0$ & $30,466.2$ & $57,317.2$ & $322,966.2$ & $136,000.0$ & $458,966.2$ & $516,283.4$ & \\
\hline \multirow{5}{*}{4.} & & Asphalt & $5,471.0$ & 289.0 & $5,760.0$ & 140.0 & 544.0 & 684.0 & $9,847.8$ & $4,566.2$ & $14,414.0$ & $16,212.0$ & $136,000.0$ & $152,212.0$ & $166,626.0$ & 95.5 \\
\hline & & Dirt & $5,048.0$ & $2,000.0$ & $7,048.0$ & 613.0 & - & 613.0 & $8,076.8$ & $22,600.0$ & $30,676.8$ & $70,985.4$ & & - $70,985.4$ & $101,662.2$ & 73.0 \\
\hline & 2006 & R.P. & $3,160.0$ & 550.0 & $3,710.0$ & 345.0 & - & 345.0 & $5,056.0$ & $3,300.0$ & $8,356.0$ & $39,951.0$ & & - $39,951.0$ & $48,307.0$ & 28.9 \\
\hline & & N.P. & $5,073.0$ & & $5,073.0$ & $1,691.0$ & & $1,691.0$ & $8,116.8$ & & $8,116.8$ & $195,817.8$ & & $195,817.8$ & $203,934.6$ & 28.2 \\
\hline & Total 4 & & $18,752.0$ & $2,839.0$ & $21,591.0$ & $2,789.0$ & 544.0 & $3,333.0$ & $31,097.4$ & $30,466.2$ & $61,563.6$ & $322,966.2$ & $136,000.0$ & $458,966.2$ & $520,529.8$ & \\
\hline \multirow{5}{*}{5.} & & Asphalt & $6,015.0$ & 289.0 & $6,304.0$ & 140.0 & 544.0 & 684.0 & $10,827.0$ & $4,566.2$ & $15,393.2$ & $16,212.0$ & $136,000.0$ & $152,212.0$ & $167,605.2$ & 95.9 \\
\hline & & Dirt & $4,504.0$ & $2,000.0$ & $6,504.0$ & 613.0 & - & 613.0 & $7,206.4$ & $22,600.0$ & $29,806.4$ & $70,985.4$ & - & & $100,791.8$ & 71.0 \\
\hline & 2007 & R.P. & $4,055.0$ & 550.0 & $4,605.0$ & 345.0 & - & 345.0 & $6,488.0$ & $3,300.0$ & $9,788.0$ & $39,951.0$ & & - $39,951.0$ & $49,739.0$ & 36.3 \\
\hline & & N.P. & $6,764.0$ & & $6,764.0$ & $1,691.0$ & - & $1,691.0$ & $10,822.4$ & - & $10,822.4$ & $195,817.8$ & & $195,817.8$ & $206,640.2$ & 35.2 \\
\hline & Total 5 & & $21,338.0$ & $2,839.0$ & $24,177.0$ & $2,789.0$ & 544.0 & $3,333.0$ & $35,343.8$ & $30,466.2$ & $65,810.0$ & $322,966.2$ & $136,000.0$ & $458,966.2$ & $524,776.2$ & \\
\hline \multirow{5}{*}{6.} & & Asphalt & $6,559.0$ & 289.0 & $6,848.0$ & 140.0 & 544.0 & 684.0 & $11,806.2$ & $4,566.2$ & $16,372.4$ & $16,212.0$ & $136,000.0$ & $152,212.0$ & $168,584.4$ & 96.2 \\
\hline & & Dirt & $3,960.0$ & $2,000.0$ & $5,960.0$ & 613.0 & - & 613.0 & $6,336.0$ & $22,600.0$ & $28,936.0$ & $70,985.4$ & & $\begin{array}{r}-70,985.4 \\
\end{array}$ & $99,921.4$ & 68.6 \\
\hline & 2008 & R.P. & $4,950.0$ & 550.0 & $5,500.0$ & 345.0 & - & 345.0 & $7,920.0$ & $3,300.0$ & $11,220.0$ & $39,951.0$ & & - $39,951.0$ & $51,171.0$ & 43.7 \\
\hline & & N.P. & $8,455.0$ & & $8,455.0$ & $1,691.0$ & & $1,691.0$ & $13,528.0$ & & $13,528.0$ & $195,817.8$ & & $195,817.8$ & $209,345.8$ & 42.3 \\
\hline & Total 6 & & $23,924.0$ & $2,839.0$ & $26,763.0$ & $2,789.0$ & 544.0 & $3,333.0$ & $39,590.2$ & $30,466.2$ & $70,056.4$ & $322,966.2$ & $136,000.0$ & $458,966.2$ & $529,022.6$ & \\
\hline \multirow{5}{*}{7.} & & Asphalt & $7,103.0$ & 289.0 & $7,392.0$ & 140.0 & 544.0 & 684.0 & $12,785.4$ & $4,566.2$ & $17,351.6$ & $16,212.0$ & $136,000.0$ & $152,212.0$ & $169,563.6$ & 96.4 \\
\hline & & Dirt & $3,416.0$ & $2,000.0$ & $5,416.0$ & 613.0 & - & 613.0 & $5,465.6$ & $22,600.0$ & $28,065.6$ & $70,985.4$ & & $\begin{array}{r}-70,985.4 \\
\end{array}$ & $99,051.0$ & 65.8 \\
\hline & 2009 & R.P. & $5,845.0$ & 550.0 & $6,395.0$ & 345.0 & - & 345.0 & $9,352.0$ & $3,300.0$ & $12,652.0$ & $39,951.0$ & & $\quad 39,951.0$ & $52,603.0$ & 51.1 \\
\hline & & N.P. & $10,146.0$ & & $10,146.0$ & $1,691.0$ & & $1,691.0$ & $16,233.6$ & & $16,233.6$ & $195,817.8$ & & $195,817.8$ & $212,051.4$ & 49.3 \\
\hline & Total 7 & & $26,510.0$ & $2,839.0$ & $29,349.0$ & $2,789.0$ & 544.0 & $3,333.0$ & $43,836.6$ & $30,466.2$ & $74,302.8$ & $322,966.2$ & $136,000.0$ & $458,966.2$ & $533,269.0$ & \\
\hline
\end{tabular}


Annex

\begin{tabular}{|c|c|c|c|c|c|c|c|c|c|c|c|c|c|c|c|c|}
\hline \multicolumn{17}{|c|}{ MDGS OPTIONS } \\
\hline No. & Year & $\begin{array}{l}\text { Type of } \\
\text { roads }\end{array}$ & \multicolumn{6}{|c|}{ Linear (in km) } & \multicolumn{3}{|c|}{ Costs } & & & & & $\%$ \\
\hline & & & \multicolumn{3}{|c|}{ Maintenance } & \multicolumn{3}{|c|}{ Investment } & \multicolumn{3}{|c|}{ Maintenance } & \multicolumn{3}{|c|}{ Investment } & \multirow{2}{*}{$\begin{array}{c}\text { Annual total } \\
\text { Total }\end{array}$} & \multirow{2}{*}{$\begin{array}{c}\text { In good } \\
\text { condition }\end{array}$} \\
\hline & & & In progress & Periodic & Total & $\begin{array}{c}\text { Rehabilit } \\
\text { ation }\end{array}$ & $\begin{array}{c}\text { New } \\
\text { works }\end{array}$ & Total & $\begin{array}{c}\text { In progress } \\
1 /\end{array}$ & $\begin{array}{c}\text { Periodic } \\
2 /\end{array}$ & Total & $\begin{array}{c}\text { Rehabilitatio } \\
\text { n 3/ }\end{array}$ & $\begin{array}{c}\text { New works } \\
4 /\end{array}$ & & & \\
\hline \multirow{5}{*}{8.} & \multirow{4}{*}{2010} & Asphalt & $7,647.0$ & 289.0 & $7,936.0$ & 140.0 & 544.0 & 684.0 & $13,764.6$ & $4,566.2$ & $18,330.8$ & $16,212.0$ & $136,000.0$ & $152,212.0$ & $170,542.8$ & 96.6 \\
\hline & & Dirt & $2,872.0$ & $2,000.0$ & $4,872.0$ & 613.0 & - & 613.0 & $4,595.2$ & $22,600.0$ & $27,195.2$ & $70,985.4$ & - & $70,985.4$ & $98,180.6$ & 62.5 \\
\hline & & R.P. & $6,740.0$ & 550.0 & $7,290.0$ & 345.0 & - & 345.0 & $10,784.0$ & $3,300.0$ & $14,084.0$ & $39,951.0$ & - & $39,951.0$ & $54,035.0$ & 58.5 \\
\hline & \multirow{2}{*}{\multicolumn{2}{|c|}{ Total 8}} & $11,837.0$ & $1,691.0$ & 13528.0 & $1,691.0$ & - & $1,691.0$ & $18,939.2$ & $10,146.0$ & $29,085.2$ & $195,817.8$ & - & $195,817.8$ & $224,903.0$ & 56.4 \\
\hline & & & $29,096.0$ & $4,530.0$ & 33626.0 & $2,789.0$ & 544.0 & $3,333.0$ & $48,083.0$ & $40,612.2$ & $88,695.2$ & $322,966.2$ & $136,000.0$ & $458,966.2$ & $547,661.4$ & \\
\hline \multirow{5}{*}{9.} & \multirow{4}{*}{2011} & Asphalt & $8,191.0$ & 289.0 & 8480.0 & 140.0 & 544.0 & 684.0 & $14,743.8$ & $4,566.2$ & $19,310.0$ & $16,212.0$ & $136,000.0$ & $152,212.0$ & $171,522.0$ & 96.8 \\
\hline & & Dirt & $2,328.0$ & $2,000.0$ & 4328.0 & 613.0 & - & 613.0 & $3,724.8$ & $22,600.0$ & $26,324.8$ & $70,985.4$ & - & $70,985.4$ & $97,310.2$ & 58.4 \\
\hline & & R.P. & $7,635.0$ & 550.0 & 8185.0 & 345.0 & - & 345.0 & $12,216.0$ & $3,300.0$ & $15,516.0$ & $39,951.0$ & - & $39,951.0$ & $55,467.0$ & 65.9 \\
\hline & \multirow{2}{*}{\multicolumn{2}{|c|}{ Total $9^{\text {N.P. }}$}} & $15,219.0$ & $1,691.0$ & 16910.0 & & - & & $24,350.4$ & $10,146.0$ & $34,496.4$ & - & - & & $34,496.4$ & 63.4 \\
\hline & & & $33,373.0$ & $4,530.0$ & $37,903.0$ & $1,098.0$ & 544.0 & $1,642.0$ & $55,035.0$ & $40,612.2$ & $95,647.2$ & $127,148.4$ & $136,000.0$ & $263,148.4$ & $358,795.6$ & \\
\hline \multirow{5}{*}{10.} & \multirow{4}{*}{2012} & Asphalt & $8,735.0$ & 289.0 & $9,024.0$ & 140.0 & 544.0 & 684.0 & $15,723.0$ & $4,566.2$ & $20,289.2$ & $16,212.0$ & $136,000.0$ & $152,212.0$ & $172,501.2$ & 97.0 \\
\hline & & Dirt & $1,784.0$ & $2,000.0$ & $3,784.0$ & 613.0 & - & 613.0 & $2,854.4$ & $22,600.0$ & $25,454.4$ & $70,985.4$ & - & $70,985.4$ & $96,439.8$ & 53.4 \\
\hline & & R.P. & $8,530.0$ & 550.0 & $9,080.0$ & 895.0 & - & 895.0 & $13,648.0$ & $3,300.0$ & $16,948.0$ & $103,641.0$ & - & $103,641.0$ & $120,589.0$ & 77.8 \\
\hline & \multirow{2}{*}{\multicolumn{2}{|c|}{ Total 10}} & $16,910.0$ & $1,691.0$ & $18,601.0$ & & - & - & $27,056.0$ & $10,146.0$ & $37,202.0$ & - & - & - & $37,202.0$ & 70.5 \\
\hline & & & $35,959.0$ & $4,530.0$ & $40,489.0$ & $1,648.0$ & 544.0 & $2,192.0$ & $59,281.4$ & $40,612.2$ & $99,893.6$ & $190,838.4$ & $136,000.0$ & $326,838.4$ & $426,732.0$ & \\
\hline \multirow{5}{*}{11.} & \multirow{4}{*}{2013} & Asphalt & $9,279.0$ & 289.0 & $9,568.0$ & 140.0 & 544.0 & 684.0 & $16,702.2$ & $4,566.2$ & 21268.4 & $16,212.0$ & $136,000.0$ & $152,212.0$ & $173,480.4$ & 97.2 \\
\hline & & Dirt & $1,240.0$ & $2,000.0$ & $3,240.0$ & 613.0 & - & 613.0 & $1,984.0$ & $22,600.0$ & 24584.0 & $70,985.4$ & - & $709,85.4$ & $95,569.4$ & 47.0 \\
\hline & & R.P. & $9,975.0$ & & $9,975.0$ & 345.0 & - & 345.0 & $15,960.0$ & & 15960.0 & $39,951.0$ & - & $399,51.0$ & $55,911.0$ & 85.2 \\
\hline & \multirow{2}{*}{\multicolumn{2}{|c|}{ Total 11}} & $18,601.0$ & $1,691.0$ & $20,292.0$ & & - & & $29,761.6$ & $10,146.0$ & $39,907.6$ & - & - & & $39,907.6$ & 77.5 \\
\hline & & & $39,095.0$ & $3,980.0$ & $43,075.0$ & $1,098.0$ & 544.0 & $1,642.0$ & $64,407.8$ & $37,312.2$ & $101,720.0$ & $127,148.4$ & $136,000.0$ & $263,148.4$ & $364,868.4$ & \\
\hline \multirow{5}{*}{12.} & \multirow{4}{*}{2014} & Asphalt & $9,823.0$ & 289.0 & $10,112.0$ & 140.0 & 544.0 & 684.0 & $17,681.4$ & $4,566.2$ & $22,247.6$ & $16,212.0$ & $136,000.0$ & $152,212.0$ & $174,459.6$ & $\overline{97.3}$ \\
\hline & & Dirt & 696.0 & $2,000.0$ & $2,696.0$ & 613.0 & - & 613.0 & $1,113.6$ & $22,600.0$ & $23,713.6$ & $70,985.4$ & - & $70,985.4$ & $94,699.0$ & 38.5 \\
\hline & & R.P. & $10,320.0$ & & $10,320.0$ & 895.0 & - & 895.0 & $16,512.0$ & & $16,512.0$ & $103,641.0$ & - & $103,641.0$ & $120,153.0$ & 92.6 \\
\hline & & N.P. & $20,292.0$ & $1,691.0$ & $21,983.0$ & & - & & $32,467.2$ & $10,146.0$ & $42,613.2$ & - & - & & $42,613.2$ & 84.6 \\
\hline & Total 1 & & $41,131.0$ & $3,980.0$ & $45,111.0$ & $1,648.0$ & 544.0 & $2,192.0$ & $67,774.2$ & $37,312.2$ & $105,086.4$ & $190,838.4$ & $136,000.0$ & $326,838.4$ & $431,924.8$ & \\
\hline & & Asphalt & $10,634.0$ & & $10,634.0$ & 140.0 & 544.0 & 684.0 & $19,141.2$ & & $19,141.2$ & $16,212.0$ & $136,000.0$ & $152,212.0$ & $171,353.2$ & 100.0 \\
\hline & & Dirt & 152.0 & $2,000.0$ & $2,152.0$ & 613.0 & - & 613.0 & 243.2 & $22,600.0$ & $22,843.2$ & $70,985.4$ & - & $70,985.4$ & $93,828.6$ & 26.8 \\
\hline 13. & 2015 & R.P. & $11,215.0$ & & $11,215.0$ & 895.0 & - & 895.0 & $17,944.0$ & & $17,944.0$ & $103,641.0$ & - & $103,641.0$ & $121,585.0$ & 100.0 \\
\hline & & N.P. & $21,983.0$ & $1,691.0$ & $23,674.0$ & & - & & $35,172.8$ & $10,146.0$ & $45,318.8$ & - & . & & $45,318.8$ & 91.6 \\
\hline & Total 1 & & $43,984.0$ & $3,691.0$ & $47,675.0$ & $1,648.0$ & 544.0 & $2,192.0$ & $72,501.2$ & $32,746.0$ & $105,247.2$ & $190,838.4$ & $136,000.0$ & $326,838.4$ & $432,085.6$ & \\
\hline & Total ger & eral & $353,902.0$ & $45,114.0$ & $399,016.0$ & $29,452.0$ & $7,072.0$ & $36,524.0$ & $584,764.4$ & $442,470.4$ & $1,027,234.8$ & $3,410,541.6$ & $1,768,000.0$ & $5,178,541.6$ & $6,205,776.4$ & \\
\hline
\end{tabular}
$\mathrm{P}=$ Main roads in rural areas

N.P. $=$ Secondary roads

$1 /$ In progress (1.8/1.6/1.6/1.6)

2/ Periodic (15.8/11.3/6.0/6.0)

3/ Rehabilitation (115.8/15.2/9.0/9.0)

4/ New works (250.0/22.0/22.0/22.0) 
Annex

ANNEX 6.3.2 : MTEF FOR PUBLIC WORKS IN ACCORDANCE WITH THE CORE SCENARIO OF THE PRSP

\begin{tabular}{|c|c|c|c|c|c|c|c|c|c|c|c|c|c|c|c|c|}
\hline \multirow{4}{*}{ No. } & \multirow{4}{*}{ Year } & \multirow{4}{*}{$\begin{array}{c}\text { Type of } \\
\text { roads }\end{array}$} & \multirow{2}{*}{\multicolumn{2}{|c|}{ Linear (in km) }} & \multirow{2}{*}{\multicolumn{6}{|c|}{ Costs }} & & & & & \multirow{2}{*}{\multicolumn{2}{|c|}{ Percentage }} \\
\hline & & & & & & & & & & & & & & & & \\
\hline & & & \multicolumn{2}{|c|}{ Maintenance } & \multicolumn{4}{|c|}{ Investment } & \multicolumn{2}{|l|}{ Maintenance } & \multicolumn{4}{|c|}{ Investment } & \multirow[t]{2}{*}{ Annual total } & \multirow{2}{*}{$\begin{array}{c}\text { In good } \\
\text { condition }\end{array}$} \\
\hline & & & In progress & Periodic & Total & Rehabilitation & $\begin{array}{l}\text { New } \\
\text { works }\end{array}$ & Total & $\begin{array}{c}\text { In progress } \\
1 /\end{array}$ & $\begin{array}{c}\text { Periodic } \\
2 /\end{array}$ & Total & $\begin{array}{c}\text { Rehabilitation } \\
3 /\end{array}$ & $\begin{array}{c}\text { New } \\
\text { works 4/ }\end{array}$ & Total & & \\
\hline \multirow{4}{*}{1.} & & Asphalt & $2,988.0$ & $1,310.0$ & $4,298.0$ & 130.0 & 127.0 & 257.0 & $5,378.4$ & $20,698.0$ & $26,076.4$ & $15,054.0$ & $31,750.0$ & $46,804.0$ & $72,880.4$ & 71.2 \\
\hline & & Dirt & $7,596.0$ & $1,100.0$ & $8,696.0$ & 160.0 & - & 160.0 & $12,153.6$ & $12,430.0$ & $24,583.6$ & $18,528.0$ & - & $18,528.0$ & $43,111.6$ & 79.1 \\
\hline & 2003 & R.P. & 475.0 & 290.0 & 765.0 & 333.0 & & 333.0 & 760.0 & $1,740.0$ & $2,500.0$ & $38,561.4$ & - & $38,561.4$ & $41,061.4$ & 6.7 \\
\hline & & N.P. & & & & $1,000.0$ & & $1,000.0$ & - & & & $115,800.0$ & - & $115,800.0$ & $115,800.0$ & 4.2 \\
\hline Total 1 & & & $11,059.0$ & $2,700.0$ & $13,759.0$ & $1,623.0$ & 127.0 & $1,750.0$ & $18,292.0$ & $34,868.0$ & $53,160.0$ & $187,943.4$ & $31,750.0$ & $219,693.4$ & $272,853.4$ & \\
\hline \multirow{4}{*}{2.} & & Asphalt & $3,115.0$ & $1,310.0$ & $4,425.0$ & 140.0 & 127.0 & 267.0 & $5,607.0$ & $20,698.0$ & $26,305.0$ & $16,212.0$ & $31,750.0$ & $47,962.0$ & $74,267.0$ & 72.1 \\
\hline & & Dirt & $7,469.0$ & $1,100.0$ & $8,569.0$ & 597.0 & - & 597.0 & $11,950.4$ & $12,430.0$ & $24,380.4$ & $69,132.6$ & - & $69,132.6$ & $93,513.0$ & 83.4 \\
\hline & 2004 & R.P. & $1,098.0$ & 290.0 & $1,388.0$ & 333.0 & - & 333.0 & $1,756.8$ & $1,740.0$ & $3,496.8$ & $38,561.4$ & - & $38,561.4$ & $42,058.2$ & 11.8 \\
\hline & & N.P. & $1,000.0$ & & $1,000.0$ & $1,000.0$ & & $1,000.0$ & $1,600.0$ & & $1,600.0$ & $115,800.0$ & - & $115,800.0$ & $117,400.0$ & 8.3 \\
\hline Total 2 & & & $12,682.0$ & $2,700.0$ & $15,382.0$ & $2,070.0$ & 127.0 & $2,197.0$ & $20,914.2$ & $34,868.0$ & $55,782.2$ & $239,706.0$ & $31,750.0$ & $271,456.0$ & $327,238.2$ & \\
\hline \multirow{4}{*}{3.} & & Asphalt & $3,242.0$ & $1,310.0$ & $4,552.0$ & 140.0 & 127.0 & 267.0 & $5,835.6$ & $20,698.0$ & $26,533.6$ & $16,212.0$ & $31,750.0$ & $47,962.0$ & $74,495.6$ & 72.8 \\
\hline & & Dirt & $7,342.0$ & $1,100.0$ & $8,442.0$ & 597.0 & - & 597.0 & $11,747.2$ & $12,430.0$ & $24,177.2$ & $69,132.6$ & - & $69,132.6$ & $93,309.8$ & 83.1 \\
\hline & 2005 & R.P. & $1,721.0$ & 290.0 & $2,011.0$ & 333.0 & - & 333.0 & $2,753.6$ & $1,740.0$ & $4,493.6$ & $38,561.4$ & - & $38,561.4$ & $43,055.0$ & 17.0 \\
\hline & & N.P. & $2,000.0$ & - & $2,000.0$ & $1,000.0$ & & $1,000.0$ & $3,200.0$ & - & $3,200.0$ & $115,800.0$ & - & $115,800.0$ & $119,000.0$ & 12.5 \\
\hline Total 3 & & & $14,305.0$ & $2,700.0$ & $17,005.0$ & $2,070.0$ & 127.0 & $2,197.0$ & $23,536.4$ & $34,868.0$ & $58,404.4$ & $239,706.0$ & $31,750.0$ & $271,456.0$ & $329,860.4$ & \\
\hline \multirow{4}{*}{4.} & & Asphalt & $3,369.0$ & $1,310.0$ & $4,679.0$ & 140.0 & 127.0 & 267.0 & $6,064.2$ & $20,698.0$ & $26,762.2$ & $16,212.0$ & $31,750.0$ & $47,962.0$ & $74,724.2$ & 73.5 \\
\hline & & Dirt & $7,215.0$ & $1,100.0$ & $8,315.0$ & 597.0 & - & 597.0 & $11,544.0$ & $12,430.0$ & $23,974.0$ & $69,132.6$ & - & $69,132.6$ & $93,106.6$ & 82.9 \\
\hline & 2006 & R.P. & $2,344.0$ & 290.0 & $2,634.0$ & 333.0 & & 333.0 & $3,750.4$ & $1,740.0$ & $5,490.4$ & $38,561.4$ & - & $38,561.4$ & $44,051.8$ & 22.1 \\
\hline & & N.P. & $3,000.0$ & & $3,000.0$ & $1,000.0$ & & $1,000.0$ & $4,800.0$ & & $4,800.0$ & $115,800.0$ & - & $115,800.0$ & $120,600.0$ & 16.7 \\
\hline Total 4 & & & $15,928.0$ & $2,700.0$ & $18,628.0$ & $2,070.0$ & 127.0 & $2,197.0$ & $26,158.6$ & $34,868.0$ & $61,026.6$ & $239,706.0$ & $31,750.0$ & $271,456.0$ & $332,482.6$ & \\
\hline \multirow{4}{*}{5.} & & Asphalt & $3,496.0$ & $1,310.0$ & $4,806.0$ & 140.0 & 127.0 & 267.0 & $6,292.8$ & $20,698.0$ & $26,990.8$ & $16,212.0$ & $31,750.0$ & $47,962.0$ & $74,952.8$ & 74.2 \\
\hline & & Dirt & $7,088.0$ & $1,100.0$ & $8,188.0$ & 597.0 & - & 597.0 & $11,340.8$ & $12,430.0$ & $23,770.8$ & $69,132.6$ & - & $69,132.6$ & $92,903.4$ & 82.7 \\
\hline & 2007 & R.P. & $2,967.0$ & 290.0 & $3,257.0$ & 333.0 & & 333.0 & $4,747.2$ & $1,740.0$ & $6,487.2$ & $38,561.4$ & - & $38,561.4$ & $45,048.6$ & 27.3 \\
\hline & & N.P. & $4,000.0$ & & $4,000.0$ & $1,000.0$ & & $1,000.0$ & $6,400.0$ & & $6,400.0$ & $115,800.0$ & - & $115,800.0$ & $122,200.0$ & 20.8 \\
\hline Total 5 & & & $17,551.0$ & $2,700.0$ & $20,251.0$ & $2,070.0$ & 127.0 & $2,197.0$ & $28,780.8$ & $34,868.0$ & $63,648.8$ & $239,706.0$ & $31,750.0$ & $271,456.0$ & $335,104.8$ & \\
\hline \multirow{4}{*}{6.} & & Asphalt & $3,623.0$ & $1,310.0$ & $4,933.0$ & 140.0 & 127.0 & 267.0 & $6,521.4$ & $20,698.0$ & $27,219.4$ & $16,212.0$ & $31,750.0$ & $47,962.0$ & $75,181.4$ & 74.8 \\
\hline & & Dirt & $6,961.0$ & $1,100.0$ & $8,061.0$ & 597.0 & - & 597.0 & $11,137.6$ & $12,430.0$ & $23,567.6$ & $69,132.6$ & - & $69,132.6$ & $92,700.2$ & 82.4 \\
\hline & 2008 & R.P. & $3,590.0$ & 290.0 & $3,880.0$ & 333.0 & & 333.0 & $5,744.0$ & $1,740.0$ & $7,484.0$ & $38,561.4$ & - & $38,561.4$ & $46,045.4$ & 32.4 \\
\hline & & N.P. & $5,000.0$ & & $5,000.0$ & $1,000.0$ & & $1,000.0$ & $8,000.0$ & & $8,000.0$ & $115,800.0$ & - & $115,800.0$ & $123,800.0$ & 25.0 \\
\hline Total 6 & & & $19,174.0$ & $2,700.0$ & $21,874.0$ & $2,070.0$ & 127.0 & $2,197.0$ & $31,403.0$ & $34,868.0$ & $66,271.0$ & $239,706.0$ & $31,750.0$ & $271,456.0$ & $337,727.0$ & \\
\hline \multirow{4}{*}{7.} & & & $3,750.0$ & $1,310.0$ & $5,060.0$ & 140.0 & 127.0 & 267.0 & $6,750.0$ & & & $16,212.0$ & $31,750.0$ & & $75,410.0$ & 75.4 \\
\hline & & Dirt & $6,834.0$ & $1,100.0$ & $7,934.0$ & 597.0 & - & 597.0 & $10,934.4$ & $12,430.0$ & $23,364.4$ & $69,132.6$ & - & $69,132.6$ & $92,497.0$ & 82.2 \\
\hline & 2009 & R.P. & $4,213.0$ & 290.0 & $4,503.0$ & 333.0 & & 333.0 & $6,740.8$ & $1,740.0$ & $8,480.8$ & $38,561.4$ & - & $38,561.4$ & $47,042.2$ & 37.5 \\
\hline & & N.P. & $6,000.0$ & & $6,000.0$ & $1,000.0$ & & $1,000.0$ & $9,600.0$ & & $9,600.0$ & $115,800.0$ & - & $115,800.0$ & $125,400.0$ & 29.2 \\
\hline Total 7 & & & $20,797.0$ & $2,700.0$ & $23,497.0$ & $2,070.0$ & 127.0 & $2,197.0$ & $34,025.2$ & $34,868.0$ & $68,893.2$ & $239,706.0$ & $31,750.0$ & $271,456.0$ & $340,349.2$ & \\
\hline
\end{tabular}


Annex

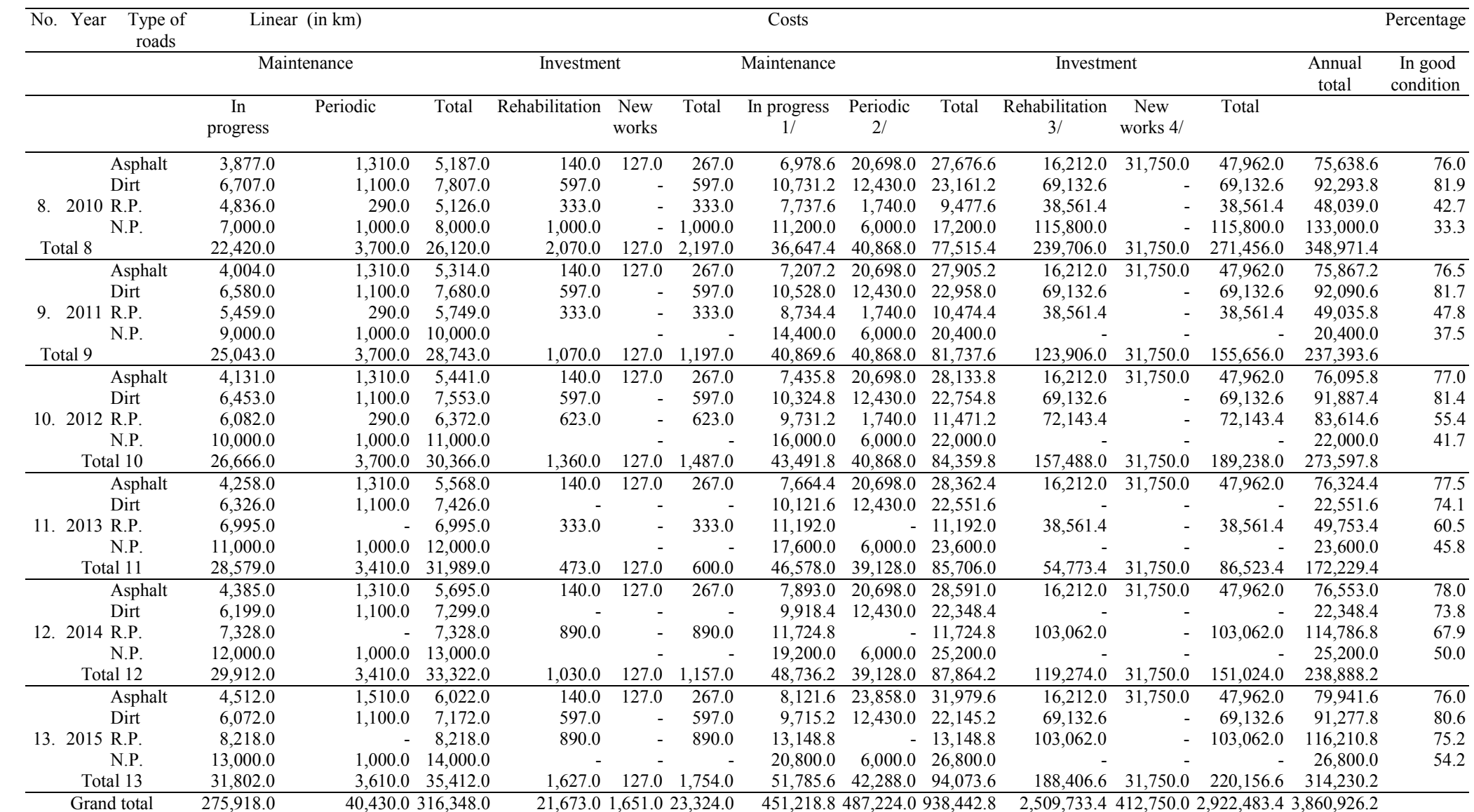

R.P $=$ Main roads in rural areas, N.P. $=$ Secondary roads

$1 /$ In progress $(1.8 / 1.6 / 1,6 / 1.6)$

3/ Rehabilitation (115.8/15.2/9.0/9.0)

4/ New works (250.0/22.0/22.0/22.0) 


\section{ANNEX 7 THE 123 PRSP MODEL}

The 123PRSP model is a simple framework to measure the impact of economic policies on poverty through two main transmission channels: Growth intensity (GDP average growth rate) and the sector distribution of growth (between traded and non-traded goods). The model is made up of several modules as follows:

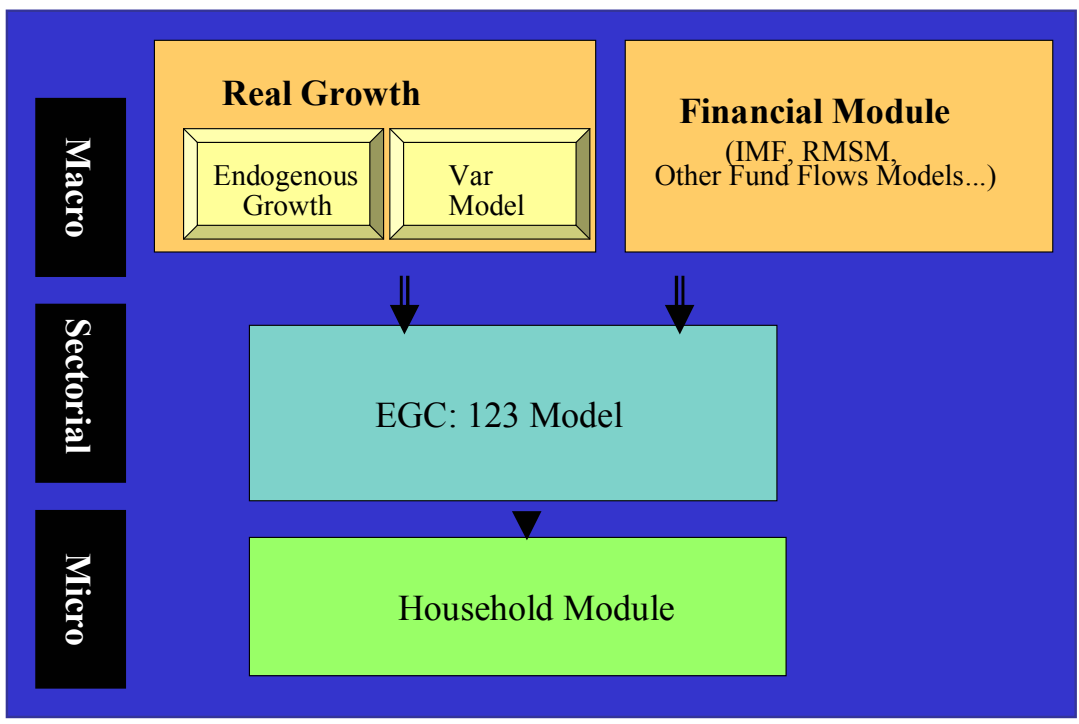

\section{Variances of the GDP growth rate are derived from:}

- Either the long-term endogenous growth model where the potential GDP growth rate depends on four key variables (i) macroeconomic policies: made up primarily of commercial policies pertaining to the degree of liberalization of trade and of fiscal and monetary policies, (ii) human capital, (iii) physical capital and notably infrastructure, and (iv)outside chocks.

\begin{tabular}{lcc}
\hline \multicolumn{2}{c}{ Long-term coefficient growth model } & \\
\hline & Coefficient & T stat \\
Structural and macro policies & -0.0153 & $(-4.02)$ \\
Prime on the black market & 0.0004 & $(3.31)$ \\
Financial Depth (\%) & -0.0014 & $(-0.21)$ \\
Inflation & -0.0087 & $(-2.36)$ \\
Real Exchange Rate & 0.0003 & $(2.40)$ \\
Secondary School attendance rate & & \\
(\%) & 0.0054 & $(2.13)$ \\
Number of telephone lines per 1000 & & \\
inhabitants & & \\
Shocks & 0.2125 & $(2.45)$ \\
Gains from terms of exchange as a & & \\
percent of GDP & -0.0029 & $(-3.28)$ \\
Interest on foreign debt as a percent & & \\
of GDP & 0.0210 & $(3.56)$ \\
OECD Country Growth & & \\
Initial Conditions & -0.0105 & $(-2.33)$ \\
Initial Revenue & 0.0236 & $(-0.41)$ \\
Intercepts & -0.0021 & $(0.60)$ \\
\hline Mute Variable, 80s & 0.0046 & \\
Mute Variable, 90s &
\end{tabular}


- Or a VAR short-term economic growth model that displays the dynamic variation (for the next five years) of the GDP growth rate in response to the timed increase (from today's date) of $1 \%$ of terms of trade or public expenditures.

\begin{tabular}{lrrrrr}
\hline & Year 1 & Year 2 & Year 3 & Year 4 & Year 5 \\
\hline $\begin{array}{l}\text { Shocks on terms of } \\
\text { exchange }(+1 \%)\end{array}$ & 0.05 & 0.13 & 0.05 & 0.007 & -0.03 \\
\hline $\begin{array}{l}\text { Variance in expenditures of } \\
\text { public spending }(+1 \%)\end{array}$ & 0.02 & 0.045 & -0.002 & -0.059 & -0.007 \\
\hline
\end{tabular}

We note that an increase in the terms of trade or of public expenditures induce an increase in the GDP. However, this effect disappears quickly.

Relative prices and factor costs GDP variances Data as well as the information contained in the macroeconomic framework provided by the "Direction de la Prevision" are inserted to the 123 model, an easy to use general equilibrium model that helps determine the evolution of relative prices and wages.

Impact on poverty and household income distribution: GDP growth and price trends data are applied to the household module built from the ECAMII survey data, in order to measure the impact of shocks and policies on spending and revenues on each decile. The table below indicates the share of household income from domestic or exported goods and the share of household consumption of domestic and imported goods.

\begin{tabular}{cccccccc}
\hline Deciles & $\begin{array}{c}\text { Expenditure } \\
\text { for } \\
\text { Domestic } \\
\text { Goods }\end{array}$ & $\begin{array}{c}\text { Expenditure } \\
\text { for } \\
\text { Exported } \\
\text { Goods }\end{array}$ & $\begin{array}{c}\text { Total } \\
\text { Expenditure }\end{array}$ & Wages & $\begin{array}{c}\text { Revenue } \\
\text { from } \\
\text { Domestic } \\
\text { Goods }\end{array}$ & $\begin{array}{c}\text { Revenue } \\
\text { From } \\
\text { Exported } \\
\text { Goods }\end{array}$ & $\begin{array}{c}\text { Total } \\
\text { Revenue }\end{array}$ \\
\hline 1 & 85.4 & 14.6 & 100.00 & 12.5 & 78.7 & 8.9 & 100.00 \\
2 & 84.0 & 16.0 & 100.00 & 11.3 & 80.8 & 7.8 & 100.00 \\
3 & 83.1 & 16.9 & 100.00 & 15.7 & 77.7 & 6.7 & 100.00 \\
4 & 82.8 & 17.2 & 100.00 & 12.5 & 78.1 & 9.4 & 100.00 \\
5 & 82.1 & 17.9 & 100.00 & 14.2 & 77.5 & 8.2 & 100.00 \\
6 & 81.9 & 18.1 & 100.00 & 22.7 & 72.4 & 5.0 & 100.00 \\
7 & 80.9 & 19.1 & 100.00 & 22.4 & 70.4 & 7.2 & 100.00 \\
8 & 80.3 & 19.7 & 100.00 & 23.5 & 70.2 & 6.3 & 100.00 \\
9 & 79.3 & 20.7 & 100.00 & 29.8 & 66.2 & 4.0 & 100.00 \\
10 & 75.5 & 24.5 & 100.00 & 41.7 & 53.9 & 4.4 & 100.00 \\
Total & 79.5 & 20.5 & 0.0 & 21.5 & 71.8 & 6.7 & 100.00 \\
\hline
\end{tabular}

Source : Growth and poverty: Growth and poverty, the 123PSRP model for Cameroon. F.Charlier, S. Devarajan, M. Mba, K. Mezama Mbida, C.N'cho Oguie, JP. Nkou, F. Tchana Tchana, 2003. 
ANNEX 8 RESULTS OF POVERTY DETERMINANTS ECONOMIC MODEL

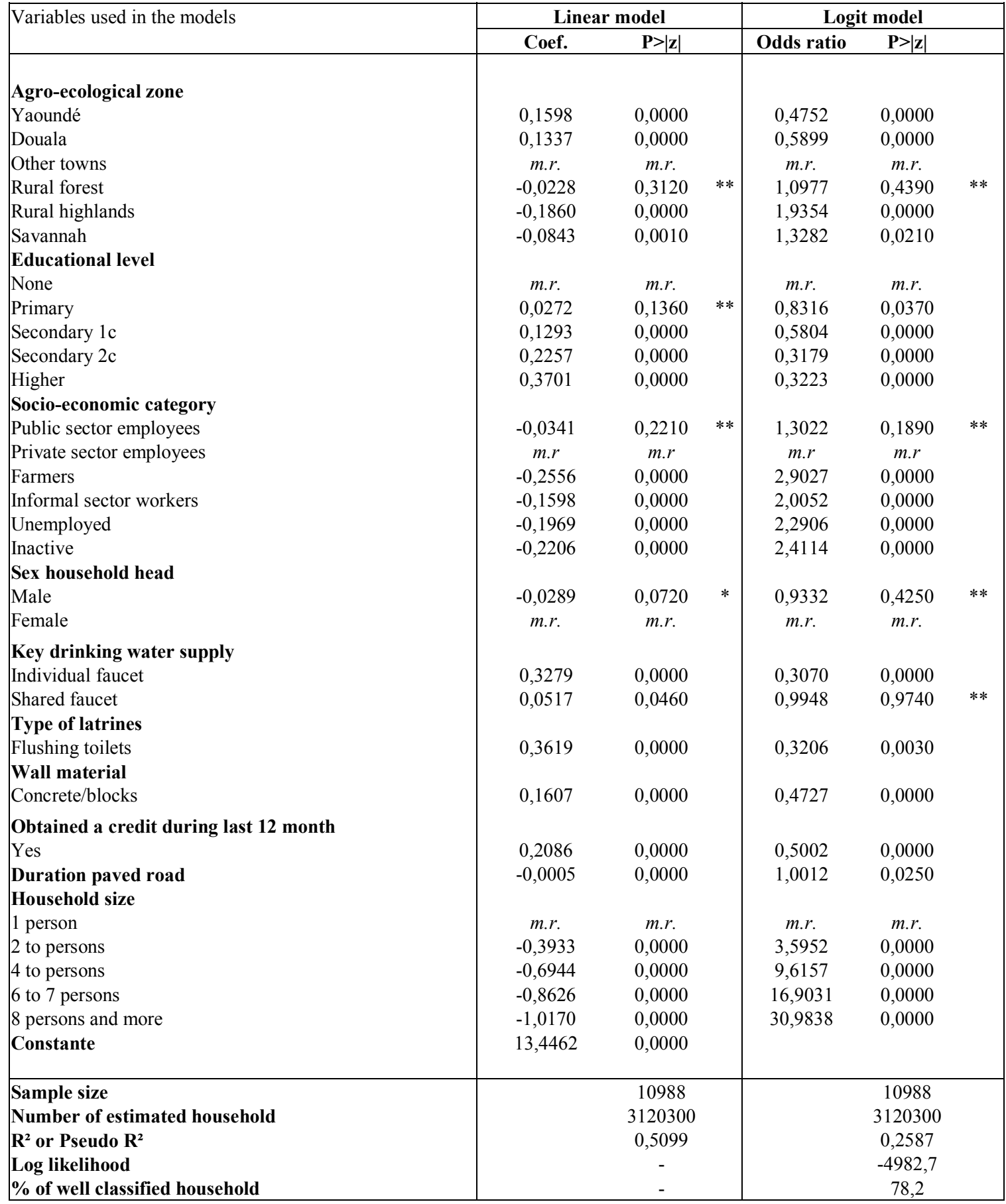

Source : ECAM II ; DSCN

$\mathrm{m} . \mathrm{r}=$ baseline modality

* not significant at the $5 \%$ level

** not significant at the $10 \%$ level 